One-Electron Oxidative and Reductive Potentials of Nitroxide

\title{
Antioxidants: A Theoretical Study
}

Jennifer L. Hodgson ${ }^{1}$, Mansoor Namazian ${ }^{1}$, Steven E. Bottle ${ }^{2}$, and Michelle L. $\operatorname{Coote}^{1^{*}}$

${ }^{1}$ Research School of Chemistry, Australian National University, Canberra, ACT 0200, Australia

${ }^{2}$ Queensland University of Technology, Brisbane, QLD, 4000, Australia

\section{SUPPORTING INFORMATION}

\footnotetext{
*Email address for correspondence, email: mcoote@rsc.anu.edu.au
} 


\section{TABLE S1: Geometries of the species in this study.}

NOTE: All species had zero imaginary frequencies, as determined from frequency calculations at the B3-LYP/6-31G(d) level

\section{$\mathrm{DBN} \bullet(1)$}

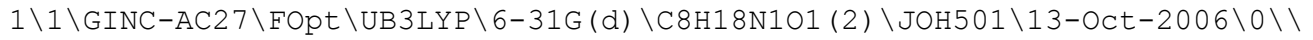

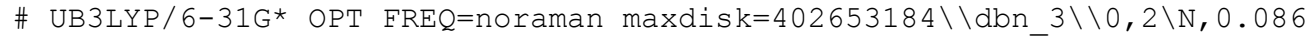
$676393,-0.0289569824,-0.2101692723 \backslash 0,1.274667638,-0.091 \overline{1} 022481,-0.7035$ $929955 \backslash C,-0.3620070154,1.3925564994,0.0618667796 \backslash C,-0.4443604176,-1.30$ $97344046,0.401084604 \backslash C,-1.9045160561,-1.5693512106,-0.0228288026 \backslash C, 0.4$ $193109189,-2.4592743379,-0.1487459585 \backslash C,-0.3080527061,-1.2802033621,1$. $9374802489 \backslash \mathrm{C},-1.8274124185,1.5272440584,0.4996489237 \backslash \mathrm{C}, 0.5674020513,1$. $9916398436,1.1380605006 \backslash \mathrm{C},-0.1819272335,2.158708748,-1.2637190109 \backslash \mathrm{H}, 0$. $0330344105,-3.4043915051,0.2486247153 \backslash H, 0.378269924,-2.4963617895,-1.2$ $407199587 \backslash \mathrm{H}, 1.4644838447,-2.3519364626,0.1430830661 \backslash \mathrm{H},-0.6115015138,-2$ $.2484948044,2.3524792362 \backslash \mathrm{H}, 0.7325158484,-1.0984501561,2.2251155619 \backslash \mathrm{H},-$ $0.9348993843,-0.513457395,2.4013852659 \backslash \mathrm{H},-2.175999689,-2.5961353611,0$. $2463819683 \backslash \mathrm{H},-2.6219763047,-0.9077145572,0.4636789761 \backslash \mathrm{H},-2.0142282526$, $-1.4666553212,-1.1076606017 \backslash \mathrm{H},-0.4537587934,3.2106275479,-1.1228525904$ $\backslash \mathrm{H}, 0.8525242997,2.0986038843,-1.6039435408 \backslash \mathrm{H},-0.827157554,1.7376825983$ $,-2.042888488 \backslash \mathrm{H}, 0.378602061,3.0660332106,1.2441109575 \backslash \mathrm{H}, 0.4077119255,1$ $.5234929233,2.1148860617 \backslash \mathrm{H}, 1.6105078889,1.8454674368,0.8463928685 \backslash \mathrm{H},-2$ $.0329500287,2.5918357933,0.6566754969 \backslash \mathrm{H},-2.5195232337,1.1700910365,-0$. $2679548001 \backslash \mathrm{H},-2.0463066024,1.0167763242,1.4409707871 \backslash \backslash$ Version=IA64L-G0 3RevD.01 $\backslash$ State $=2-A \backslash H F=-445.599719 \backslash \mathrm{S} 2=0.753796 \backslash \mathrm{S} 2-1=0 . \backslash \mathrm{S} 2 \mathrm{~A}=0.75001 \backslash \mathrm{RMSD}$ $=5.976 \mathrm{e}-09 \backslash \mathrm{RMSF}=9.462 \mathrm{e}-06 \backslash \mathrm{Thermal}=0 . \backslash \mathrm{Dipole}=-0.9066189,0.0568884,0.568$ $8285 \backslash P G=C 01 \quad[X(C 8 H 18 \mathrm{~N} 101)] \backslash \backslash @$

\section{DBN+}

$1 \backslash 1 \backslash G I N C-A C 49 \backslash F O p t \backslash U B 3 L Y P \backslash 6-31 G(d) \backslash C 8 H 18 N 101(1+) \backslash J O H 501 \backslash 09-M a r-2007 \backslash 0 \backslash$

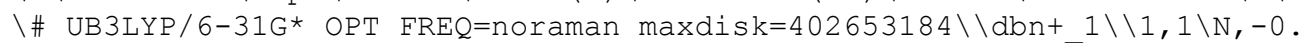
$0042964547,-0.4640911268,-0.3440839536 \backslash 0,-0.0063114352,-1.5075558274,-$ $0.9261992153 \backslash C, 1.4082171759,0.1345514116,-0.0345823722 \backslash C,-1.4140787958$ $, 0.1175895775,0.0055503091 \backslash \mathrm{C},-1.6970766832,1.1750772459,-1.0914418397 \backslash$ $C,-2.408936201,-1.0432615077,-0.1075357927 \backslash C,-1.4417931569,0.719045612$ $4,1.4171896161 \backslash \mathrm{C}, 1.4399276944,1.6519432342,-0.2613604018 \backslash \mathrm{C}, 1.692899724$ $4,-0.2473561958,1.440145791 \backslash C, 2.3989763451,-0.5726161038,-0.9664272148$ $\backslash \mathrm{H},-3.3917473442,-0.6581547133,0.1775790328 \backslash \mathrm{H},-2.4812584251,-1.4380453$ $065,-1.1225055931 \backslash \mathrm{H},-2.1566656244,-1.8624518096,0.5725600958 \backslash \mathrm{H},-2.4562$ $533261,1.0969947863,1.5761258324 \backslash \mathrm{H},-1.2506020477,-0.0373238582,2.18278$ $66008 \backslash \mathrm{H},-0.7621937871,1.5600031951,1.5548387293 \backslash \mathrm{H},-2.74328919,1.473563$ $1941,-0.9710172704 \backslash \mathrm{H},-1.0802801693,2.0687229369,-0.997500915 \backslash \mathrm{H},-1.5838$ $460743,0.7525515459,-2.09410579 \backslash \mathrm{H}, 3.3834586903,-0.1308522611,-0.789880$ $8801 \backslash \mathrm{H}, 2.468689957,-1.6441834455,-0.7712865946 \backslash \mathrm{H}, 2.1447804411,-0.42151$ $38801,-2.0199045982 \backslash \mathrm{H}, 2.7400913681,0.0089001412,1.62958268 \backslash \mathrm{H}, 1.0787219$ $622,0.3019657517,2.1535434665 \backslash \mathrm{H}, 1.5772267195,-1.3225723113,1.605077755$ $3 \backslash \mathrm{H}, 2.4559717198,1.9821963747,-0.0250494123 \backslash \mathrm{H}, 1.2470330552,1.908834841$ $,-1.3061461768 \backslash \mathrm{H}, 0.7636238621,2.2116384987,0.3847081116 \backslash \backslash$ Version=IA64L $-\mathrm{G} 03$ RevD.01 $\backslash$ State $=1-\mathrm{A} \backslash \mathrm{HF}=-445.3525674 \backslash \mathrm{S} 2=0 . \backslash \mathrm{S} 2-1=0 . \backslash \mathrm{S} 2 \mathrm{~A}=0 . \backslash \mathrm{RMSD}=7.629 \mathrm{e}$ $-09 \backslash \mathrm{RMSF}=1.282 \mathrm{e}-05 \backslash \mathrm{Thermal}=0 . \backslash \mathrm{Dipole}=0.0009776,0.4349213,0.2427312 \backslash \mathrm{PG}=$ $\mathrm{C} 01[\mathrm{X}(\mathrm{C} 8 \mathrm{H} 18 \mathrm{~N} 101)] \backslash \backslash \mathrm{Q}$

\section{DBN-}

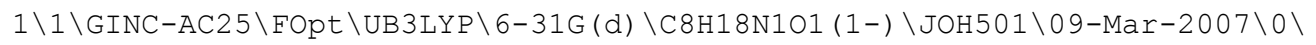

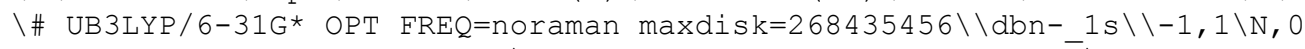
$.0055745199,0.0034204148,0 . \backslash 0,1.4223765029,0.0188206187,0 . \backslash C,-0.437931$ $362,0.5840042602,1.3212026835 \backslash C,-0.437931362,0.5840042602,-1.321202683$ $5 \backslash C,-1.9632109004,0.6446740652,1.5202356843 \backslash C,-1.9632109004,0.64467406$ 
$52,-1.5202356843 \backslash C, 0.1071460067,-0.4410255286,2.3455577416 \backslash C, 0.1071460$ $067,-0.4410255286,-2.3455577416 \backslash \mathrm{C}, 0.2059960991,1.9552684571,1.62211717$ $35 \backslash \mathrm{C}, 0.2059960991,1.9552684571,-1.6221171735 \backslash \mathrm{H},-2.4341302061,-0.280565$ $8976,1.1647555096 \backslash \mathrm{H},-2.4341302061,-0.2805658976,-1.1647555096 \backslash \mathrm{H},-2.433$ $4724831,1.4920464048,1.0114196735 \backslash \mathrm{H},-2.4334724831,1.4920464048,-1.0114$ $196735 \backslash \mathrm{H},-2.1918820596,0.7477917814,2.5908878282 \backslash \mathrm{H},-2.1918820596,0.747$ $7917814,-2.5908878282 \backslash \mathrm{H},-0.3945023415,-1.4076628811,2.2047872653 \backslash \mathrm{H},-0$. $3945023415,-1.4076628811,-2.2047872653 \backslash \mathrm{H},-0.0570801117,-0.1044489466,3$ $.3802900669 \backslash \mathrm{H},-0.0570801117,-0.1044489466,-3.3802900669 \backslash \mathrm{H}, 1.1697689433$ $,-0.5724988183,2.134354579 \backslash \mathrm{H}, 1.1697689433,-0.5724988183,-2.134354579 \backslash \mathrm{H}$ $, 0.2215382949,2.182222355,2.6985449442 \backslash \mathrm{H}, 0.2215382949,2.182222355,-2.6$ $985449442 \backslash \mathrm{H},-0.3173103025,2.7772987021,1.1171462906 \backslash \mathrm{H},-0.3173103025,2$. $7772987021,-1.1171462906 \backslash \mathrm{H}, 1.2317149113,1.87696553,1.2409071242 \backslash \mathrm{H}, 1.23$ $17149113,1.87696553,-1.2409071242 \backslash \backslash$ Version=IA64L-G03RevD.01 \State=1-A' $\backslash \mathrm{HF}=-445.586548 \backslash \mathrm{S} 2=0 . \backslash \mathrm{S} 2-1=0 . \backslash \mathrm{S} 2 \mathrm{~A}=0 . \backslash \mathrm{RMSD}=3.200 \mathrm{e}-09 \backslash \mathrm{RMSF}=9.465 \mathrm{e}-06 \backslash \mathrm{The}$ $\mathrm{rmal}=0 . \backslash \mathrm{Dipole}=-1.5914622,0.655151,0 . \backslash \mathrm{PG}=\mathrm{CS} \quad[\mathrm{SG}(\mathrm{N} 101), \mathrm{X}(\mathrm{C} 8 \mathrm{H} 18)] \backslash \backslash @$

\section{TEMPO• (2)}

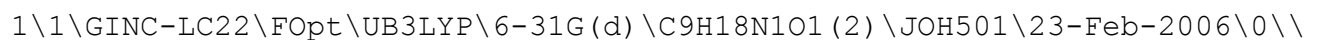

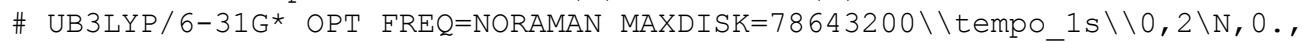
$-0.7434209403,-0.2055568746 \backslash 0,0 .,-2.0200229887,-0.0536930208 \backslash C, 0 ., 2.12$ $91122715,0.0202793419 \backslash \mathrm{H}, 0 ., 3.1640205144,-0.3430045373 \backslash \mathrm{H}, 0 ., 2.188129084$ $3,1.1160257969 \backslash \mathrm{C}, 1.2471385297,1.4014636869,-0.4850966271 \backslash \mathrm{C},-1.24713852$ $97,1.4014636869,-0.4850966271 \backslash C, 1.3316319116,-0.0694129504,-0.02866995$ $47 \backslash \mathrm{C},-1.3316319116,-0.0694129504,-0.0286699547 \backslash \mathrm{C}, 2.3409037477,-0.82546$ $55447,-0.9084597306 \backslash \mathrm{C},-2.3409037477,-0.8254655447,-0.9084597306 \backslash \mathrm{C}, 1.76$ $16105348,-0.1821678854,1.4497256469 \backslash \mathrm{C},-1.7616105348,-0.1821678854,1.44$ $97256469 \backslash \mathrm{H}, 2.1613559084,1.9128572411,-0.159270535 \backslash \mathrm{H},-2.1613559084,1.91$ $28572411,-0.159270535 \backslash \mathrm{H}, 1.2461755503,1.4303666274,-1.5834226174 \backslash \mathrm{H},-1.2$ $461755503,1.4303666274,-1.5834226174 \backslash \mathrm{H}, 2.0267037652,-0.8094181301,-1.9$ $575486217 \backslash \mathrm{H},-2.0267037652,-0.8094181301,-1.9575486217 \backslash \mathrm{H}, 3.3238440946,-$ $0.3468698717,-0.8343424769 \backslash \mathrm{H},-3.3238440946,-0.3468698717,-0.8343424769$ $\backslash \mathrm{H}, 2.4264203456,-1.8666991071,-0.5927537894 \backslash \mathrm{H},-2.4264203456,-1.8666991$ $071,-0.5927537894 \backslash \mathrm{H}, 2.801008229,0.1459162404,1.5646144668 \backslash \mathrm{H},-2.8010082$ $29,0.1459162404,1.5646144668 \backslash \mathrm{H}, 1.1414032892,0.4302012714,2.1114446532 \backslash$ $\mathrm{H},-1.1414032892,0.4302012714,2.1114446532 \backslash \mathrm{H}, 1.6868923818,-1.2242044777$ $, 1.7731554021 \backslash \mathrm{H},-1.6868923818,-1.2242044777,1.7731554021 \backslash \backslash$ Version=IA32 L-G03RevC.02\State=2-A $\backslash \mathrm{HF}=-483.7197701 \backslash \mathrm{S} 2=0.753695 \backslash \mathrm{S} 2-1=0 . \backslash \mathrm{S} 2 \mathrm{~A}=0.7500$ $09 \backslash \mathrm{RMSD}=3.459 \mathrm{e}-09 \backslash \mathrm{RMSF}=7.184 \mathrm{e}-06 \backslash \mathrm{Dipole}=0 ., 1.1367503,0.065858 \backslash \mathrm{PG}=\mathrm{CS} \quad[\mathrm{S}$ $\mathrm{G}(\mathrm{C} 1 \mathrm{H} 2 \mathrm{~N} 1 \mathrm{O} 1), \mathrm{X}(\mathrm{C} 8 \mathrm{H} 16)] \backslash \backslash @$

\section{TEMPO+}

$1 \backslash 1 \backslash G I N C-L C 42 \backslash F O p t \backslash U B 3 L Y P \backslash 6-31 G(d) \backslash C 9 H 18 N 101(1+) \backslash J O H 501 \backslash 24-F e b-2006 \backslash 0 \backslash$

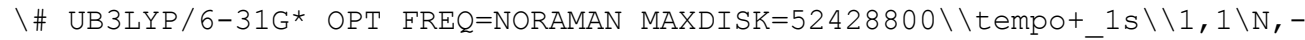
$0.115588,-0.000000109,-0.774504 \backslash 0,-0.318335,-0.000000275,-\overline{1} .953284 \backslash \mathrm{C},-$ $0.219139,0.0000002972,2.111088 \backslash \mathrm{H},-0.734081,0.0000004332,3.077235 \backslash \mathrm{H}, 0.8$ $52363,0.0000003295,2.340458 \backslash \mathrm{C},-0.620907,1.2537501878,1.3339488235 \backslash \mathrm{C},-0$ $.620907,-1.2537498122,1.3339491765 \backslash C, 0.013359,1.3649629898,-0.07270519$ $22 \backslash C, 0.013359,-1.3649630102,-0.0727048078 \backslash C,-0.706984,2.3996978673,-0$. $9425803378 \backslash \mathrm{C},-0.706984,-2.3996981327,-0.9425796622 \backslash \mathrm{C}, 1.531029,1.683659$ $9976,-0.017087237 \backslash \mathrm{C}, 1.531029,-1.6836600024,-0.017086763 \backslash \mathrm{H},-0.335708,2$. $1629352638,1.8739796955 \backslash \mathrm{H},-0.335708,-2.1629347362,1.8739803045 \backslash \mathrm{H},-1.71$ $2203,1.2837311725,1.2249448193 \backslash \mathrm{H},-1.712203,-1.2837308275,1.2249451807 \backslash$ $\mathrm{H},-1.747945,2.1181178412,-1.1280242982 \backslash \mathrm{H},-1.747945,-2.1181181588,-1.12$ $80237018 \backslash \mathrm{H},-0.704662,3.3458059446,-0.393637471 \backslash \mathrm{H},-0.704662,-3.34580605$ $54,-0.393636529 \backslash \mathrm{H},-0.20819,2.5598447324,-1.9004783604 \backslash \mathrm{H},-0.20819,-2.55$ $98452676,-1.9004776396 \backslash \mathrm{H}, 1.616726,2.7249390434,0.3080066164 \backslash \mathrm{H}, 1.616726$ $,-2.7249389566,0.3080073836 \backslash \mathrm{H}, 2.077456,1.0630220976,0.6930448503 \backslash \mathrm{H}, 2.0$ $77456,-1.0630219024,0.6930451497 \backslash \mathrm{H}, 1.991694,1.5998518584,-1.0055132252$ $\backslash \mathrm{H}, 1.991694,-1.5998521416,-1.0055127748 \backslash \backslash$ Version=IA32L-G03RevC.02\Stat $\mathrm{e}=1-\mathrm{A}^{\prime} \backslash \mathrm{HF}=-483.4708163 \backslash \mathrm{S} 2=0 . \backslash \mathrm{S} 2-1=0 . \backslash \mathrm{S} 2 \mathrm{~A}=0 . \backslash \mathrm{RMSD}=8.063 \mathrm{e}-09 \backslash \mathrm{RMSF}=3.820 \mathrm{e}$ $-06 \backslash$ Dipole $=0 ., 0.319844,0.1755 \backslash \mathrm{PG}=\mathrm{CS} \quad[\mathrm{SG}(\mathrm{C} 1 \mathrm{H} 2 \mathrm{~N} 101), \mathrm{X}(\mathrm{C} 8 \mathrm{H} 16)] \backslash \backslash @$ 


\section{TEMPO-}

$1 \backslash 1 \backslash G I N C-L C 48 \backslash F O p t \backslash U B 3 L Y P \backslash 6-31 G(d) \backslash C 9 H 18 N 101(1-) \backslash J O H 501 \backslash 24-F e b-2006 \backslash 0 \backslash$ \\# UB3LYP/6-31G* OPT FREQ=NORAMAN MAXDISK=52428800 \\empo- $1 \mathrm{~s} \backslash \backslash-1,1 \backslash \mathrm{N}$, $0 .,-0.709025629,-0.4802223634 \backslash 0,0 .,-2.0560677285,-0.064917 \overline{3} 106 \backslash \mathrm{C}, 0 ., 2$. $1586732984,0.1206189342 \backslash \mathrm{H}, 0 ., 3.2163551228,-0.187846366 \backslash \mathrm{H}, 0 ., 2.16052024$ $77,1.2191576584 \backslash \mathrm{C}, 1.2483664161,1.4465067722,-0.4077320628 \backslash \mathrm{C},-1.2483664$ $161,1.4465067722,-0.4077320628 \backslash C, 1.2745393011,-0.0557005618,-0.0522898$ $223 \backslash C,-1.2745393011,-0.0557005618,-0.0522898223 \backslash C, 2.3623619525,-0.7549$ $331641,-0.893213159 \backslash \mathrm{C},-2.3623619525,-0.7549331641,-0.893213159 \backslash \mathrm{C}, 1.609$ $1301022,-0.2988337615,1.4401523685 \backslash C,-1.6091301022,-0.2988337615,1.440$ $1523685 \backslash \mathrm{H}, 2.1643057897,1.9319030354,-0.0361494841 \backslash \mathrm{H},-2.1643057897,1.93$ $19030354,-0.0361494841 \backslash \mathrm{H}, 1.2587304195,1.5422588333,-1.5040958054 \backslash \mathrm{H},-1$. $2587304195,1.5422588333,-1.5040958054 \backslash \mathrm{H}, 2.1721228621,-0.5882006717,-1$. $9611527659 \backslash \mathrm{H},-2.1721228621,-0.5882006717,-1.9611527659 \backslash \mathrm{H}, 3.3714312361$, $-0.3907814813,-0.6480728405 \backslash \mathrm{H},-3.3714312361,-0.3907814813,-0.648072840$ $5 \backslash \mathrm{H}, 2.279974674,-1.8272549262,-0.6990674352 \backslash \mathrm{H},-2.279974674,-1.82725492$ $62,-0.6990674352 \backslash \mathrm{H}, 2.6884273315,-0.2293295217,1.639320404 \backslash \mathrm{H},-2.6884273$ $315,-0.2293295217,1.639320404 \backslash \mathrm{H}, 1.101884768,0.4004519988,2.1151124264 \backslash$ $\mathrm{H},-1.101884768,0.4004519988,2.1151124264 \backslash \mathrm{H}, 1.2558437026,-1.3198799399$, $1.6355366194 \backslash \mathrm{H},-1.2558437026,-1.3198799399,1.6355366194 \backslash \backslash$ Version=IA32L $-\mathrm{G} 03 \mathrm{RevC} .02 \backslash$ State $=1-\mathrm{A}^{\prime} \backslash \mathrm{HF}=-483.7070615 \backslash \mathrm{S} 2=0 . \backslash \mathrm{S} 2-1=0 . \backslash \mathrm{S} 2 \mathrm{~A}=0 . \backslash \mathrm{RMSD}=3.231$ $e-09 \backslash \operatorname{RMSF}=8.917 e-06 \backslash \mathrm{Dipole}=0 ., 2.1130666,0.1624655 \backslash \mathrm{PG}=\mathrm{CS} \quad[\mathrm{SG}(\mathrm{C} 1 \mathrm{H} 2 \mathrm{~N} 101)$, $\mathrm{X}(\mathrm{C} 8 \mathrm{H} 16)] \backslash \backslash @$

\section{4-carboxy-TEMPO•(3)}

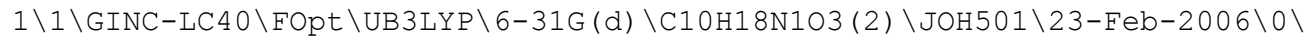
\\# UB3LYP/6-31G* OPT FREQ=NORAMAN MAXDISK=134217728\\ctempo $2 \backslash \backslash 0,2 \backslash N, 1$ $.5608959815,-0.2267743091,0.0354640909 \backslash 0,2.844667028,-0.1690103243,0.0$ $368956644 \backslash \mathrm{C},-1.2849477982,0.2315447854,0.0263207472 \backslash \mathrm{C},-2.7648766263,-0$ $.1044897368,0.0126064542 \backslash \mathrm{H},-1.2117380851,1.3251750066,-0.0158565277 \backslash \mathrm{C}$, $-0.5957862939,-0.3657399092,-1.2161468652 \backslash C,-0.6037574013,-0.318493150$ $6,1.2799879338 \backslash \mathrm{C}, 0.9054989298,-0.026127983,-1.3000312906 \backslash \mathrm{C}, 0.896843808$ $6,0.0208921079,1.3604512826 \backslash C, 1.5859762139,-0.9890379673,-2.2875654329$ $\backslash C, 1.5728423324,-0.9067390419,2.3839188797 \backslash C, 1.1352653197,1.4273116693$ $,-1.7659773278 \backslash \mathrm{C}, 1.1300638591,1.4896001838,1.7753765616 \backslash \mathrm{H},-1.081488495$ $6,-0.0155496648,-2.1329862244 \backslash \mathrm{H},-1.0943042799,0.0587001282,2.184118625$ $1 \backslash \mathrm{H},-0.7163990747,-1.4565955339,-1.1821920245 \backslash \mathrm{H},-0.7371611817,-1.40642$ $15146,1.2910812247 \backslash \mathrm{H}, 1.4816274141,-2.0255512056,-1.9499295087 \backslash \mathrm{H}, 1.4766$ $157704,-1.953761509,2.0782741711 \backslash \mathrm{H}, 1.1197905683,-0.8963966114,-3.27467$ $08345 \backslash \mathrm{H}, 1.0943569755,-0.7864661207,3.3620445914 \backslash \mathrm{H}, 2.6500272049,-0.7618$ $871433,-2.3725927139 \backslash \mathrm{H}, 2.6348572041,-0.6717468692,2.474544889 \backslash \mathrm{H}, 0.8130$ $765668,1.543894516,-2.806825487 \backslash \mathrm{H}, 0.8074472317,1.6419591982,2.81138141$ $8 \backslash \mathrm{H}, 0.5836539982,2.1536333496,-1.1610309247 \backslash \mathrm{H}, 0.5799653964,2.195919087$ $7,1.1456329584 \backslash \mathrm{H}, 2.2006839902,1.6645197349,-1.7008627546 \backslash \mathrm{H}, 2.195972128$ $4,1.7225125881,1.7030380797 \backslash 0,-3.3238542136,-0.9017693437,0.7328805865$ $\backslash 0,-3.4247414822,0.5958637154,-0.9462473734 \backslash \mathrm{H},-4.3545599231,0.30248460$ $28,-0.9032942705 \backslash \backslash$ Version=IA32L-G03RevC.02 $\backslash$ State $=2-A \backslash H F=-672.2853009 \backslash \mathrm{S}$ $2=0.753685 \backslash S 2-1=0 . \backslash \mathrm{S} 2 \mathrm{~A}=0.750009 \backslash \mathrm{RMSD}=6.001 \mathrm{e}-09 \backslash \mathrm{RMSF}=3.337 \mathrm{e}-06 \backslash \mathrm{Dipole}=-$ $0.8184605,0.487631,-0.4111102 \backslash \mathrm{PG}=\mathrm{C} 01 \quad[\mathrm{X}(\mathrm{C} 10 \mathrm{H} 18 \mathrm{~N} 103)] \backslash \backslash \mathrm{Q}$

\section{4-carboxy-TEMPO+}

$1 \backslash 1 \backslash G I N C-A C 49 \backslash F O p t \backslash U B 3 L Y P \backslash 6-31 G(d) \backslash C 10 H 18 N 103(1+) \backslash J O H 501 \backslash 09-M a r-2006 \backslash 0$ $\backslash \backslash \#$ UB3LYP/6-31G* OPT FREQ=NORAMAN MAXDISK $=134217728 \backslash \backslash$ ctempo+ $1 \backslash \backslash 1,1 \backslash \mathrm{N}$ $, 1.5626860867,0.0525438394,-0.2602996323 \backslash 0,2.6800528209,0.0909 \overline{2} 17027,-$ $0.6836445621 \backslash C,-1.2744896954,-0.0801385671,0.2921876793 \backslash C,-2.765514141$ $3,-0.2029728749,-0.0281570282 \backslash \mathrm{H},-1.2085945954,0.0118296334,1.383731718$ $7 \backslash \mathrm{C},-0.6792480097,1.1754480655,-0.3641241528 \backslash \mathrm{C},-0.5346057187,-1.324282$ $0699,-0.2001865495 \backslash C, 0.8227559894,1.3906604877,-0.0768520949 \backslash C, 0.97904$ $18888,-1.3278686986,0.0963871294 \backslash C, 1.442472076,2.3971955772,-1.0527460$ $805 \backslash C, 1.7149651444,-2.3707263774,-0.7524891876 \backslash C, 1.0948132404,1.833851$ $9986,1.3852334167 \backslash \mathrm{C}, 1.2980231472,-1.5494684632,1.5997042124 \backslash \mathrm{H},-1.19928$ $29314,2.0745945554,-0.0217392029 \backslash \mathrm{H},-0.9523972151,-2.2279289852,0.25335$ 
$27784 \backslash \mathrm{H},-0.8241351354,1.1186181353,-1.4503103472 \backslash \mathrm{H},-0.6914776392,-1.42$ $96038571,-1.2794303877 \backslash \mathrm{H}, 1.4033900546,2.0354106254,-2.0846740826 \backslash \mathrm{H}, 1.6$ $379559921,-2.1474642403,-1.8208356897 \backslash \mathrm{H}, 0.8526300183,3.3163420325,-0.9$ $930473866 \backslash \mathrm{H}, 1.2326775474,-3.3359818622,-0.5736403217 \backslash \mathrm{H}, 2.4768813964,2$. $6379728855,-0.8004384632 \backslash \mathrm{H}, 2.7690505013,-2.4591651788,-0.4827443645 \backslash \mathrm{H}$, $0.7253945008,2.8600945689,1.472529007 \backslash \mathrm{H}, 1.044085067,-2.5908781309,1.81$ $89405197 \backslash \mathrm{H}, 0.5761663285,1.2250084286,2.1261590555 \backslash \mathrm{H}, 0.7145288662,-0.91$ $29608396,2.2654476748 \backslash \mathrm{H}, 2.1662995006,1.8393397929,1.6038523024 \backslash \mathrm{H}, 2.363$ $3233325,-1.4098459651,1.803381741 \backslash 0,-3.2712365085,-1.1479859604,-0.583$ $0308638 \backslash 0,-3.4379675395,0.8848575261,0.3956981285 \backslash \mathrm{H},-4.3813719057,0.75$ $42709115,0.175637188 \backslash \backslash$ Version=IA64 L-G03RevC.02 $\backslash$ State $=1-A \backslash H F=-672.03158$ $5 \backslash S 2=0 . \backslash S 2-1=0 . \backslash S 2 A=0 . \backslash R M S D=3.352 e-09 \backslash R M S F=3.731 e-06 \backslash D i p o l e=1.2411796$, $0.5653958,0.4968617 \backslash \mathrm{PG}=\mathrm{C} 01 \quad[\mathrm{X}(\mathrm{C} 10 \mathrm{H} 18 \mathrm{~N} 103)] \backslash \backslash @$

\section{4-carboxy-TEMPO-}

$1 \backslash 1 \backslash$ GINC-LC1 49 \FOpt \UB3LYP \6-31G (d) \C10H18N1O3 (1-) \JOH501 \27-Feb-2006

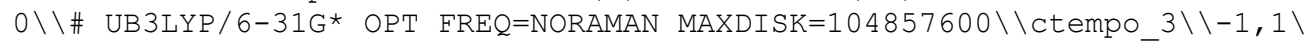
$\mathrm{N}, 1.5355452486,0.0168220254,-0.4807526752 \backslash 0,2.8912557082,0.1192610104$, $-0.1155753548 \backslash \mathrm{C},-1.3038361752,-0.0920604864,0.2355837855 \backslash \mathrm{C},-2.77369102$ $24,-0.153859429,-0.0961526883 \backslash \mathrm{H},-1.2258425668,0.0161380094,1.323039292$ $5 \backslash \mathrm{C},-0.6780127068,1.1586120962,-0.4268334594 \backslash \mathrm{C},-0.5443646623,-1.334111$ $3267,-0.242588347 \backslash \mathrm{C}, 0.8295393615,1.2824500318,-0.1214742492 \backslash \mathrm{C}, 0.965365$ $4318,-1.2522852629,0.0646668421 \backslash C, 1.4350095123,2.3392413631,-1.0689279$ $014 \backslash \mathrm{C}, 1.6916889683,-2.3643692501,-0.7190838698 \backslash \mathrm{C}, 1.1027877871,1.742443$ $7528,1.3312483514 \backslash \mathrm{C}, 1.2748252079,-1.460803587,1.5674669543 \backslash \mathrm{H},-1.202730$ $2542,2.0678109217,-0.1023123426 \backslash \mathrm{H},-0.9697601022,-2.2417698087,0.209499$ $3116 \backslash \mathrm{H},-0.8141872231,1.0651052717,-1.5128833643 \backslash \mathrm{H},-0.6894876228,-1.421$ $1593994,-1.3263059987 \backslash \mathrm{H}, 1.2392247032,2.0612012857,-2.1119814505 \backslash \mathrm{H}, 1.48$ $19450543,-2.2627082582,-1.7909888105 \backslash \mathrm{H}, 1.0295838127,3.3443118112,-0.88$ $17220696 \backslash \mathrm{H}, 1.3882611848,-3.3681069671,-0.3872316056 \backslash \mathrm{H}, 2.5162966604,2.3$ $212833794,-0.9118469311 \backslash \mathrm{H}, 2.7635686512,-2.2144050348,-0.568249604 \backslash \mathrm{H}, 0$. $9840404815,2.8284853388,1.4522887578 \backslash \mathrm{H}, 1.2690342524,-2.5235223285,1.84$ $84700299 \backslash \mathrm{H}, 0.4579172403,1.2547300914,2.0716217888 \backslash \mathrm{H}, 0.5756112279,-0.93$ $96408876,2.2318735857 \backslash \mathrm{H}, 2.1472997049,1.4546671111,1.5095746934 \backslash \mathrm{H}, 2.281$ $7164494,-1.0427911477,1.6973218188 \backslash 0,-3.3015172295,-0.7430037372,-1.01$ $91724234 \backslash 0,-3.5317835003,0.595250113,0.7661194568 \backslash \mathrm{H},-4.4408184353,0.51$ $90099326,0.4206956864 \backslash \backslash$ Version=IA32L-G03RevC.02\State $=1-A \backslash H F=-672.2802$ $698 \backslash \mathrm{S} 2=0 . \backslash \mathrm{S} 2-1=0 . \backslash \mathrm{S} 2 \mathrm{~A}=0 . \backslash \mathrm{RMSD}=7.877 \mathrm{e}-09 \backslash \mathrm{RMSF}=1.165 \mathrm{e}-05 \backslash \mathrm{Dipole}=-3.04225$ $12,0.2052632,0.5633032 \backslash \mathrm{PG}=\mathrm{CO} 1 \quad[\mathrm{X}(\mathrm{C} 10 \mathrm{H} 18 \mathrm{~N} 1 \mathrm{O} 3)] \backslash \backslash \mathrm{C}$

\section{4-amino-TEMPO• (4)}

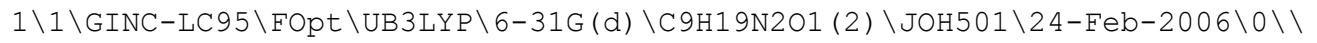

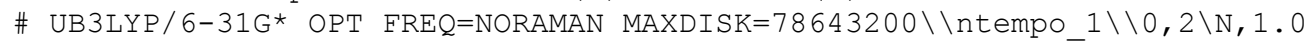
$25731725,-0.2936895048,-0.0052493203 \backslash 0,2.3111388737,-0.30 \overline{6} 8730089,-0.0$ $123838237 \backslash \mathrm{C},-1.8151823173,0.2953301141,0.0017965305 \backslash \mathrm{N},-3.2622869159,0$. $0528195581,-0.0694742084 \backslash \mathrm{H},-1.6893573865,1.3856417523,-0.0050383438 \backslash \mathrm{C}$, $-1.1314423866,-0.2924895128,1.2458898079 \backslash C,-1.1460418993,-0.2974449382$ $,-1.2390515774 \backslash \mathrm{C}, 0.3866610098,-0.033772605,1.3281094562 \backslash \mathrm{C}, 0.3695684162$ $,-0.0348680397,-1.3315746 \backslash C, 1.023891024,-1.0092716788,2.3317910178 \backslash \mathrm{C}, 0$ $.9981868004,-1.0081475972,-2.3428874266 \backslash \mathrm{C}, 0.6947975591,1.4150026581,1$. $7645605643 \backslash \mathrm{C}, 0.6702388437,1.414532831,-1.7706342334 \backslash \mathrm{H},-1.3104336846,-1$ $.3780080303,1.2450257141 \backslash \mathrm{H},-1.3189190915,-1.3842821366,-1.2320533568 \backslash \mathrm{H}$ $,-1.596007368,0.101243257,2.1598842823 \backslash \mathrm{H},-1.6300918946,0.0922778699,-2$ $.1412095538 \backslash \mathrm{H}, 0.86649674,-2.0459665898,2.0154964182 \backslash \mathrm{H}, 0.852274109,-2.0$ $453481745,-2.0228324442 \backslash \mathrm{H}, 0.5667214393,-0.8745100071,3.3183928048 \backslash \mathrm{H}, 0$. $5243678446,-0.8777632279,-3.3220737465 \backslash \mathrm{H}, 2.0985783354,-0.8345999169,2$. $4094451188 \backslash \mathrm{H}, 2.0703202313,-0.8269014259,-2.4378930452 \backslash \mathrm{H}, 0.1786808876,2$ $.156043899,1.1467040145 \backslash \mathrm{H}, 0.1557895429,2.1544480024,-1.1500069789 \backslash \mathrm{H}, 0$. $3828494761,1.5681795661,2.804043021 \backslash \mathrm{H}, 0.3485065648,1.5657318075,-2.807$ $1406818 \backslash \mathrm{H}, 1.7709887901,1.5955754844,1.6929123535 \backslash \mathrm{H}, 1.7465312496,1.5979$ $142728,-1.7076731316 \backslash \mathrm{H},-3.4378079378,-0.9520895216,-0.0244577722 \backslash \mathrm{H},-3$. $7167748018,0.450259427,0.7526133825 \backslash \backslash$ Version=IA32L-G03RevC.02\State=2$\mathrm{A} \backslash \mathrm{HF}=-539.0627624 \backslash \mathrm{S} 2=0.753691 \backslash \mathrm{S} 2-1=0 . \backslash \mathrm{S} 2 \mathrm{~A}=0.750009 \backslash \mathrm{RMSD}=8.174 \mathrm{e}-09 \backslash \mathrm{RMSF}$ 

$\backslash \backslash 0$

\section{4-amino-TEMPO+}

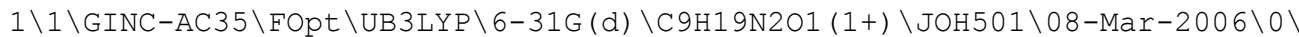

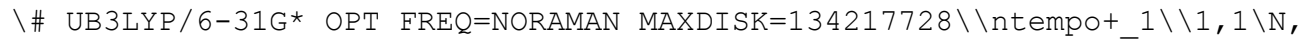
$-1.050536303,0.0241979485,-0.2454597256 \backslash 0,-2.1792956024,0.0470979701,-$ $0.6397050463 \backslash \mathrm{C}, 1.8284843725,-0.0460480776,0.2306924813 \backslash \mathrm{N}, 3.2408481826$, $-0.1714616361,-0.0962627139 \backslash \mathrm{H}, 1.767018812,-0.0451240147,1.3255255148 \backslash \mathrm{C}$ $, 1.1497393974,1.2239228978,-0.3221048389 \backslash \mathrm{C}, 1.0943909499,-1.2758331656$, $-0.3166198382 \backslash \mathrm{C},-0.3541558701,1.3733942334,0.0036868162 \backslash \mathrm{C},-0.409909428$ $2,-1.3526198686,0.0122184114 \backslash C,-1.0335845767,2.4141683807,-0.89359227 \backslash$ $\mathrm{C},-1.1351804188,-2.3691981406,-0.8768729577 \backslash \mathrm{C},-0.6096131807,1.71709408$ $35,1.4962653406 \backslash \mathrm{C},-0.6796393142,-1.673901022,1.507533113 \backslash \mathrm{H}, 1.276501316$ $8,1.2424019935,-1.4130514733 \backslash \mathrm{H}, 1.2181166745,-1.307313197,-1.4077961487$ $\backslash \mathrm{H}, 1.6427458175,2.1224779318,0.0659487226 \backslash \mathrm{H}, 1.5606069991,-2.1842178793$ $, 0.0765764601 \backslash \mathrm{H},-0.9981290673,2.1223811484,-1.9476274487 \backslash \mathrm{H},-1.09275238$ $27,-2.0839644637,-1.9324172144 \backslash \mathrm{H},-0.4813889568,3.3517999598,-0.7828913$ $408 \backslash \mathrm{H},-0.619890715,-3.3272214857,-0.7640548137 \backslash \mathrm{H},-2.0726103424,2.59431$ $46369,-0.6106191548 \backslash \mathrm{H},-2.1790930594,-2.5057298765,-0.5880823064 \backslash \mathrm{H},-0.0$ $576210164,1.0807956815,2.1881709041 \backslash \mathrm{H},-0.0994343996,-1.057451029,2.194$ $3235022 \backslash \mathrm{H},-0.2692700102,2.7470911547,1.6392216954 \backslash \mathrm{H},-0.3814910472,-2.7$ $159495175,1.6569728823 \backslash \mathrm{H},-1.6761619562,1.6763055071,1.7346958183 \backslash \mathrm{H},-1$. $7432909267,-1.5868315936,1.7463365988 \backslash \mathrm{H}, 3.406399168,-0.0704824758,-1.0$ $965472033 \backslash \mathrm{H}, 3.7987351679,0.5349036451,0.3777749057 \backslash \backslash$ Version=IA64L-G03R evC.02 $\backslash$ State $=1-A \backslash H F=-538.8125307 \backslash S 2=0 . \backslash S 2-1=0 . \backslash S 2 A=0 . \backslash R M S D=4.844 \mathrm{e}-09 \backslash R$ $\mathrm{MSF}=1.697 \mathrm{e}-05 \backslash \mathrm{Dipole}=-0.2701788,0.4559988,0.1034585 \backslash \mathrm{PG}=\mathrm{C} 01 \quad[\mathrm{X}(\mathrm{C} 9 \mathrm{H} 19 \mathrm{~N} 20$ 1) $] \backslash \backslash @$

\section{4-amino-TEMPO-}

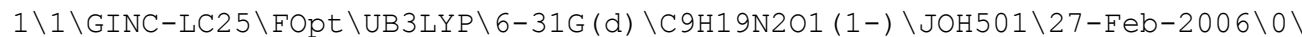
\\# UB3LYP/6-31G* OPT FREQ=NORAMAN MAXDISK=104857600\\ctempo- $1 \backslash \backslash-1,1 \backslash \mathrm{N}$ $,-1.0098173152,0.023101243,-0.4768136949 \backslash 0,-2.3678192098,0.0 \overline{5} 77229077$, $-0.1028347873 \backslash \mathrm{C}, 1.8511181324,-0.0547317311,0.2030573052 \backslash \mathrm{N}, 3.2963743416$ $,-0.153588641,-0.1198554536 \backslash \mathrm{H}, 1.7891728732,-0.057032014,1.2990583629 \backslash \mathrm{C}$ $, 1.1723235223,1.2075046831,-0.3416823522 \backslash C, 1.1130867381,-1.2765099731$, $-0.3416744808 \backslash C,-0.3374592931,1.2808280336,-0.0337740616 \backslash C,-0.39863837$ $21,-1.2654390175,-0.0312331863 \backslash C,-0.9795510544,2.3820310524,-0.9026183$ $041 \backslash \mathrm{C},-1.096747552,-2.3354963831,-0.8956465317 \backslash \mathrm{C},-0.6213117747,1.63129$ $29529,1.4484097378 \backslash \mathrm{C},-0.6972684354,-1.5970734303,1.4520710839 \backslash \mathrm{H}, 1.3080$ $538869,1.2087934519,-1.4353232163 \backslash \mathrm{H}, 1.2413171246,-1.282573489,-1.43671$ $31898 \backslash \mathrm{H}, 1.6774685844,2.1086542686,0.0447110949 \backslash \mathrm{H}, 1.5772033232,-2.19615$ $77658,0.0413745283 \backslash \mathrm{H},-0.7753925287,2.1874737553,-1.9632259218 \backslash \mathrm{H},-0.890$ $5905385,-2.1501770163,-1.9574977212 \backslash \mathrm{H},-0.6067883915,3.3842624672,-0.64$ $36189112 \backslash \mathrm{H},-0.7676276073,-3.3532072517,-0.6388509042 \backslash \mathrm{H},-2.0599557333,2$ $.31850069,-0.7492009524 \backslash \mathrm{H},-2.1721988746,-2.2237874968,-0.7358702275 \backslash \mathrm{H}$, $0.0374585314,1.1074572846,2.1504758017 \backslash \mathrm{H},-0.008859381,-1.1085682425,2$. $1512591201 \backslash \mathrm{H},-0.5284444667,2.7090638716,1.6459722892 \backslash \mathrm{H},-0.6601311902,-$ $2.6776851204,1.6511694951 \backslash \mathrm{H},-1.6585453367,1.3075430643,1.6066441168 \backslash \mathrm{H}$, $-1.715601489,-1.2198635722,1.6138458812 \backslash \mathrm{H}, 3.3777340086,-0.1727802314,-$ $1.1397680503 \backslash \mathrm{H}, 3.7390702333,0.7272747484,0.1534654815 \backslash \backslash$ Version=IA32L-G $03 R e v C .02 \backslash$ State $=1-A \backslash H F=-539.0530205 \backslash S 2=0 . \backslash S 2-1=0 . \backslash S 2 A=0 . \backslash R M S D=6.984 e-0$ $9 \backslash \mathrm{RMSF}=4.105 e-06 \backslash \mathrm{Dipole}=2.3139852,0.3488758,-0.0276345 \backslash \mathrm{PG}=\mathrm{C} 01 \quad[\mathrm{X}(\mathrm{C} 9 \mathrm{H} 19$ $\mathrm{N} 2 \mathrm{O} 1)] \backslash \backslash \mathrm{C}$

\section{4-methoxy-TEMPO•(5)}

$1 \backslash 1 \backslash$ GINC-LC105\FOpt \UB3LYP \6-31G (d) \C10H20N1O2 (2) \JOH501 \25-Feb-2006\0

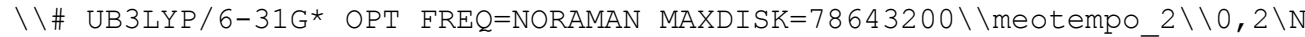
$, 1.3585491165,-0.2492576778,-0.1508625252 \backslash 0,2.6381164736,-0.1 \overline{5} 2005346$, $-0.2212800277 \backslash C,-1.4991460211,0.115773042,0.0193960371 \backslash 0,-2.8578309582$ 
$,-0.312855145,0.0056279391 \backslash \mathrm{H},-1.4756295599,1.2174210825,0.048159308 \backslash \mathrm{C}$, $-0.7254633947,-0.4651199278,1.206521788 \backslash \mathrm{C},-0.8676967298,-0.3939807547$, $-1.2710637202 \backslash \mathrm{C}, 0.7687887718,-0.0855350465,1.2207535465 \backslash \mathrm{C}, 0.6157405742$ $,-0.0127059277,-1.4350495717 \backslash C, 1.5312184721,-1.0396424876,2.1556288239$ $\backslash C, 1.2597608207,-0.9138719846,-2.5020178462 \backslash C, 0.9857050204,1.368464458$ $1,1.6936628966 \backslash \mathrm{C}, 0.7844062733,1.4648563738,-1.8506404924 \backslash \mathrm{H},-0.82786057$ $93,-1.5568048357,1.157470694 \backslash \mathrm{H},-0.9712146198,-1.4866493803,-1.27813921$ $57 \backslash \mathrm{H},-1.164238883,-0.146260601,2.1593359062 \backslash \mathrm{H},-1.4295751233,-0.0164284$ $194,-2.1327861688 \backslash \mathrm{H}, 1.4389283702,-2.0743741256,1.8092684113 \backslash \mathrm{H}, 1.202643$ $8513,-1.966027573,-2.2038746589 \backslash \mathrm{H}, 1.1166966197,-0.9745731112,3.1676825$ $99 \backslash \mathrm{H}, 0.7299013933,-0.7943839964,-3.4534502124 \backslash \mathrm{H}, 2.5912967308,-0.781332$ $9626,2.186615147 \backslash \mathrm{H}, 2.3103050349,-0.6532666137,-2.6427956099 \backslash \mathrm{H}, 0.384352$ $8791,2.0863983139,1.127482084 \backslash \mathrm{H}, 0.2551054437,2.1502098359,-1.181443393$ $3 \backslash \mathrm{H}, 0.7151511534,1.4634637715,2.7514138529 \backslash \mathrm{H}, 0.3934756504,1.6160004409$ $,-2.8630546719 \backslash \mathrm{H}, 2.0400717847,1.6340516214,1.5771576637 \backslash \mathrm{H}, 1.8462375943$ $, 1.7262307642,-1.8430776144 \backslash \mathrm{C},-3.6994152823,0.3627793109,0.9179422692 \backslash$ $\mathrm{H},-4.7134701893,-0.0073318539,0.7448538171 \backslash \mathrm{H},-3.4321578971,0.169101825$ $, 1.9674866955 \backslash \mathrm{H},-3.6855386209,1.4521411558,0.7521473676 \backslash \backslash$ Version=IA32L $-\mathrm{G} 03 \mathrm{RevC} .02 \backslash \mathrm{State}=2-\mathrm{A} \backslash \mathrm{HF}=-598.2354827 \backslash \mathrm{S} 2=0.753693 \backslash \mathrm{S} 2-1=0 . \backslash \mathrm{S} 2 \mathrm{~A}=0.750009$ $\backslash \mathrm{RMSD}=5.642 \mathrm{e}-09 \backslash \mathrm{RMSF}=2.970 \mathrm{e}-06 \backslash \mathrm{Dipole}=-0.9460963,0.419715,0.3468205 \backslash \mathrm{PG}$ $=\mathrm{C} 01 \quad[\mathrm{X}(\mathrm{C} 10 \mathrm{H} 20 \mathrm{~N} 1 \mathrm{O} 2)] \backslash \backslash \mathrm{Q}$

\section{4-methoxy-TEMPO+}

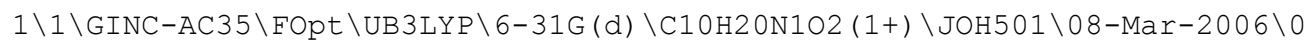

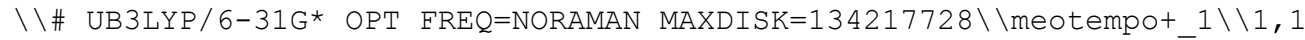
$\backslash \mathrm{N},-1.3754816053,0.1909318601,-0.1947948729 \backslash 0,-2.5148719456,0.3 \overline{9} 771471$ $18,-0.4906400143 \backslash C, 1.4709634871,-0.3378171171,0.0907928337 \backslash 0,2.7342467$ $796,-0.6617400506,-0.4389049592 \backslash \mathrm{H}, 1.5179701351,-0.3281116887,1.1917223$ $937 \backslash \mathrm{C}, 0.9795381371,1.020316279,-0.4373396989 \backslash \mathrm{C}, 0.5248613166,-1.4357724$ $394,-0.3968729251 \backslash C,-0.4499717794,1.4115126903,-0.0144491186 \backslash C,-0.9473$ $825265,-1.2731940698,0.0241833103 \backslash \mathrm{C},-1.0112215518,2.5425932818,-0.8845$ $300295 \backslash C,-1.8771542589,-2.1543288557,-0.8187110219 \backslash C,-0.5512524572,1.7$ $978758224,1.4861347732 \backslash \mathrm{C},-1.1775341124,-1.5556170208,1.5340363985 \backslash \mathrm{H}, 1$. $0419044357,0.9938005596,-1.531793347 \backslash \mathrm{H}, 0.5912740086,-1.4751098958,-1.4$ $906804131 \backslash \mathrm{H}, 1.6354417479,1.8308049028,-0.1028177455 \backslash \mathrm{H}, 0.8648226762,-2$. $408245764,-0.0269122574 \backslash \mathrm{H},-1.0939403836,2.2401408975,-1.9327923461 \backslash \mathrm{H},-$ $1.8522943344,-1.8698273977,-1.8749445776 \backslash \mathrm{H},-0.3079382136,3.3783023531$, $-0.8284154708 \backslash \mathrm{H},-1.5137389385,-3.1826601172,-0.7370795368 \backslash \mathrm{H},-1.9855455$ $206,2.891270057,-0.5367041052 \backslash \mathrm{H},-2.90993406,-2.1267216134,-0.466321447$ $\backslash \mathrm{H},-0.0747425665,1.0774872468,2.1517510141 \backslash \mathrm{H},-0.4696765651,-1.03960215$ $44,2.1832397672 \backslash \mathrm{H},-0.0308560747,2.7542090349,1.5952422086 \backslash \mathrm{H},-1.0378438$ $483,-2.6320997222,1.6709570092 \backslash \mathrm{H},-1.5925085735,1.9417700131,1.78790970$ $49 \backslash \mathrm{H},-2.1991365899,-1.3045201698,1.8327264323 \backslash \mathrm{C}, 3.8457577871,-0.054883$ $8479,0.2152309166 \backslash \mathrm{H}, 4.7388219979,-0.4634769133,-0.2598105448 \backslash \mathrm{H}, 3.85203$ $93976,1.0372983668,0.0979283293 \backslash \mathrm{H}, 3.8596295852,-0.3031366433,1.2858662$

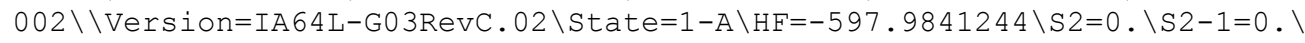
$\mathrm{S} 2 \mathrm{~A}=0 . \backslash \mathrm{RMSD}=8.135 \mathrm{e}-09 \backslash \mathrm{RMSF}=2.028 \mathrm{e}-06 \backslash \mathrm{Dipole}=-0.717227,0.3415474,0.5720$ $302 \backslash P G=C 01 \quad[X(C 10 H 20 N 1 O 2)] \backslash \backslash @$

\section{4-methoxy-TEMPO-}

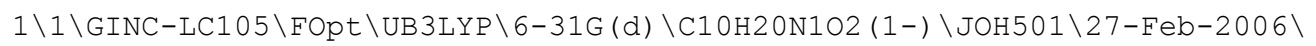
$0 \backslash \backslash \#$ UB3LYP/6-31G* OPT FREQ=NORAMAN MAXDISK=104857600 \\meotempo- $1 \backslash \backslash-1$ $, 1 \backslash \mathrm{N},-1.3561089501,0.1880295301,-0.4310432027 \backslash 0,-2.6543458626,0 . \overline{4} 48287$ $8388,0.0490315902 \backslash \mathrm{C}, 1.4774264955,-0.362482897,0.0347593886 \backslash 0,2.7996635$ $234,-0.7067768164,-0.4382603924 \backslash \mathrm{H}, 1.5121726753,-0.3569872028,1.1359340$ $986 \backslash \mathrm{C}, 1.0009179778,0.9932511269,-0.4834816134 \backslash \mathrm{C}, 0.5245493127,-1.445201$ $5576,-0.4504466251 \backslash \mathrm{C},-0.448948485,1.3129317356,-0.0544440306 \backslash \mathrm{C},-0.9360$ $989274,-1.1858360804,-0.022599321 \backslash \mathrm{C},-0.9636286873,2.5097834385,-0.8799$ $73908 \backslash \mathrm{C},-1.8648198488,-2.1224138904,-0.8224031487 \backslash \mathrm{C},-0.5504829696,1.69$ $9433008,1.4426363067 \backslash \mathrm{C},-1.1710859329,-1.4705917427,1.4821312861 \backslash \mathrm{H}, 1.05$ $13132358,0.9576237879,-1.5805829773 \backslash \mathrm{H}, 0.5796385868,-1.4634824501,-1.54$ $77580961 \backslash \mathrm{H}, 1.6659586134,1.8038820707,-0.1510051223 \backslash \mathrm{H}, 0.8621354821,-2.4$ $273566828,-0.0905115716 \backslash \mathrm{H},-0.883979035,2.2859943099,-1.9511233079 \backslash \mathrm{H},-1$ 
$.7149392953,-1.9661545597,-1.8979459213 \backslash \mathrm{H},-0.4056866552,3.4328466348,-$ $0.6633600246 \backslash \mathrm{H},-1.6864903079,-3.1817168887,-0.5857152369 \backslash \mathrm{H},-2.02251560$ $02,2.6308506573,-0.6373238082 \backslash \mathrm{H},-2.8919255213,-1.8386543464,-0.5793717$ $239 \backslash \mathrm{H}, 0.0732066346,1.0772944969,2.0950690494 \backslash \mathrm{H},-0.3617572043,-1.105101$ $8977,2.1247604066 \backslash \mathrm{H},-0.2739164805,2.7485288654,1.6208968369 \backslash \mathrm{H},-1.29711$ $65836,-2.543469082,1.6863063188 \backslash \mathrm{H},-1.6094832735,1.5428635242,1.6878997$ $629 \backslash \mathrm{H},-2.0979914413,-0.9314051539,1.7182242368 \backslash \mathrm{C}, 3.8354384031,-0.00396$ $39997,0.1952587881 \backslash \mathrm{H}, 4.7825247095,-0.4040342426,-0.1866302084 \backslash \mathrm{H}, 3.8120$ $786373,1.0791095997,-0.0074592254 \backslash \mathrm{H}, 3.8173901593,-0.138381176,1.292206$ $6147 \backslash \backslash$ Version=IA32L-G03RevC.02 $\backslash$ State $=1-A \backslash H F=-598.2270669 \backslash S 2=0 . \backslash S 2-1=0$. $\backslash \mathrm{S} 2 \mathrm{~A}=0 . \backslash \mathrm{RMSD}=2.363 \mathrm{e}-09 \backslash \mathrm{RMSF}=1.260 \mathrm{e}-05 \backslash \mathrm{Dipole}=2.8827092,-0.1897178,0.31$ $6875 \backslash \mathrm{PG}=\mathrm{CO} 1 \quad[\mathrm{X}(\mathrm{C} 10 \mathrm{H} 20 \mathrm{~N} 102)] \backslash \backslash @$

\section{4-hydroxy-TEMPO•(6)}

$1 \backslash 1 \backslash G I N C-L C 151 \backslash F O p t \backslash U B 3 L Y P \backslash 6-31 G(d) \backslash C 9 H 18 N 102$ (2) \JOH501 \27-Feb-2006\0

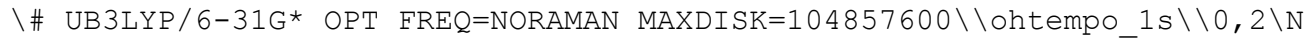
$, 1.0254691088,0 .,-0.2529401447 \backslash 0,2.3102405844,0 .,-0.21833582 \overline{1} 4 \backslash \mathrm{C},-1.82$ $49937794,0 ., 0.2211166731 \backslash 0,-3.2232762137,0 .,-0.0587455764 \backslash \mathrm{H},-1.7747618$ $716,0.1 .315027019 \backslash \mathrm{H},-3.3251891239,0 .,-1.0247426078 \backslash \mathrm{C},-1.1360419068,1$. $2447195679,-0.338331613 \backslash \mathrm{C},-1.1360419068,-1.2447195679,-0.338331613 \backslash \mathrm{C}, 0$ $.3694892873,1.3310321062,-0.0166536613 \backslash C, 0.3694892873,-1.3310321062,-0$ $.0166536613 \backslash \mathrm{C}, 1.0414114204,2.3395563969,-0.9632262732 \backslash \mathrm{C}, 1.0414114204,-$ $2.3395563969,-0.9632262732 \backslash \mathrm{C}, 0.6143161204,1.7662705834,1.4443798454 \backslash \mathrm{C}$, $0.6143161204,-1.7662705834,1.4443798454 \backslash \mathrm{H},-1.2673452647,1.2393822518,-$ $1.431271053 \backslash \mathrm{H},-1.2673452647,-1.2393822518,-1.431271053 \backslash \mathrm{H},-1.6332436663$ $, 2.1462157954,0.037304709 \backslash \mathrm{H},-1.6332436663,-2.1462157954,0.037304709 \backslash \mathrm{H}$, $0.932337534,2.0227844944,-2.0059434455 \backslash \mathrm{H}, 0.932337534,-2.0227844944,-2$. $0059434455 \backslash \mathrm{H}, 0.5711507667,3.3223189568,-0.8480968702 \backslash \mathrm{H}, 0.5711507667,-3$ $.3223189568,-0.8480968702 \backslash \mathrm{H}, 2.1066346595,2.4249919232,-0.741180947 \backslash \mathrm{H}, 2$ $.1066346595,-2.4249919232,-0.741180947 \backslash \mathrm{H}, 0.2912137596,2.8038489574,1.5$ $849999169 \backslash \mathrm{H}, 0.2912137596,-2.8038489574,1.5849999169 \backslash \mathrm{H}, 0.069772793,1.14$ $63596346,2.1629892268 \backslash \mathrm{H}, 0.069772793,-1.1463596346,2.1629892268 \backslash \mathrm{H}, 1.682$ $3873621,1.6989828613,1.6693125482 \backslash \mathrm{H}, 1.6823873621,-1.6989828613,1.66931$ $25482 \backslash \backslash$ Version=IA32L-G03RevC.02 \State $=2-A^{\prime} \backslash \mathrm{HF}=-558.9281808 \backslash \mathrm{S} 2=0.753684$ $\backslash \mathrm{S} 2-1=0 . \backslash \mathrm{S} 2 \mathrm{~A}=0.750009 \backslash \mathrm{RMSD}=3.244 \mathrm{e}-09 \backslash \mathrm{RMSF}=4.161 \mathrm{e}-06 \backslash \mathrm{Dipole}=-0.5867701$, $0 .,-0.2403593 \backslash \mathrm{PG}=\mathrm{CS} \quad[\mathrm{SG}(\mathrm{C} 1 \mathrm{H} 2 \mathrm{~N} 102), \mathrm{X}(\mathrm{C} 8 \mathrm{H} 16)] \backslash \backslash @$

\section{4-hydroxy-TEMPO+}

$1 \backslash 1 \backslash G I N C-A C 33 \backslash F O p t \backslash U B 3 L Y P \backslash 6-31 G(d) \backslash C 9 H 18 N 102(1+) \backslash J O H 501 \backslash 12-M a r-2006 \backslash 0 \backslash$ ॥ U UB3LYP/6-31G* OPT FREQ=NORAMAN MAXDISK=134217728 \/ohtempo+ $2 \backslash \backslash 1,1 \backslash \mathrm{N}$ $, 1.0400869929,-0.2422727566,-0.0064375429 \backslash 0,2.1732058537,-0.6 \overline{2} 13660622$ $,-0.0164862433 \backslash \mathrm{C},-1.8267356701,0.2454496774,0.0096235859 \backslash 0,-3.16198191$ $42,-0.2108309627,-0.0630670462 \backslash \mathrm{H},-1.7929411394,1.3452407871,0.00888101$ $35 \backslash \mathrm{H},-3.6970105071,0.2403075425,0.6093035285 \backslash \mathrm{C},-1.1439468173,-0.305739$ $8846,-1.2410268035 \backslash C,-1.125225259,-0.3130618912,1.2565460924 \backslash C, 0.35733$ $69335,0.0118266903,-1.3659620357 \backslash C, 0.3805627835,0.0031720751,1.3652590$ $131 \backslash \mathrm{C}, 1.0412895849,-0.8924413713,-2.3979652855 \backslash \mathrm{C}, 1.0807147761,-0.90797$ $97776,2.3800687249 \backslash \mathrm{C}, 0.6336826389,1.5025187634,-1.7033489255 \backslash \mathrm{C}, 0.66221$ $23223,1.4911815554,1.7079945425 \backslash \mathrm{H},-1.2920279069,-1.3922177875,-1.25217$ $85277 \backslash \mathrm{H},-1.2722177327,-1.3995629895,1.2619931088 \backslash \mathrm{H},-1.6371602604,0.087$ $6387001,-2.1354662361 \backslash \mathrm{H},-1.5931927497,0.0733605525,2.1696173776 \backslash \mathrm{H}, 0.98$ $52517828,-1.9461653611,-2.1086219648 \backslash \mathrm{H}, 1.0230415082,-1.9596016113,2.08$ $35107696 \backslash \mathrm{H}, 0.5043305393,-0.7724939272,-3.343159285 \backslash \mathrm{H}, 0.5587542216,-0.7$ $967330647,3.3347583806 \backslash \mathrm{H}, 2.0862550625,-0.6222203015,-2.5606746013 \backslash \mathrm{H}, 2$. $1276128456,-0.6368085478,2.529194357 \backslash \mathrm{H}, 0.2998401235,1.6524785651,-2.73$ $44960721 \backslash \mathrm{H}, 0.3399246615,1.6372284064,2.7434333924 \backslash \mathrm{H}, 0.085275055,2.1991$ $377947,-1.0686596136 \backslash \mathrm{H}, 0.1104033036,2.192366239,1.0814533609 \backslash \mathrm{H}, 1.70275$ $43532,1.727795195,-1.6560353974 \backslash \mathrm{H}, 1.7313586161,1.7141802821,1.65150207$

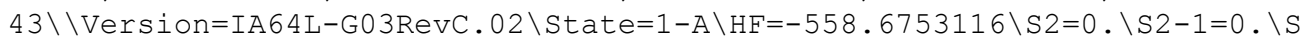
$2 \mathrm{~A}=0 . \backslash \mathrm{RMSD}=7.149 \mathrm{e}-09 \backslash \mathrm{RMSF}=8.614 \mathrm{e}-05 \backslash \mathrm{Dipole}=0.4185713,0.6265413,0.39886$ $15 \backslash \mathrm{PG}=\mathrm{C} 01 \quad[\mathrm{X}(\mathrm{C} 9 \mathrm{H} 18 \mathrm{~N} 102)] \backslash \backslash \mathrm{Q}$ 


\section{4-hydroxy-TEMPO-}

$1 \backslash 1 \backslash G I N C-L C 152 \backslash$ FOpt \UB3LYP \6-31G (d) \C9H18N1O2 (1-) \JOH501 \27-Feb-2006\0

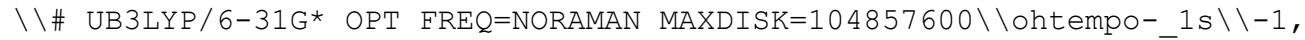
$1 \backslash \mathrm{N}, 1.0005064747,0 .,-0.4749904957 \backslash 0,2.3587052228,0 \ldots,-0.1015229566 \backslash \mathrm{C},-1$ $.8521250065,0 ., 0.2000239233 \backslash 0,-3.2619451054,0 .,-0.116423478 \backslash \mathrm{H},-1.83269$ $08199,0 ., 1.2949092182 \backslash \mathrm{H},-3.3050052819,0 .,-1.0867114026 \backslash \mathrm{C},-1.1524676146$ $, 1.2441448353,-0.339528651 \backslash C,-1.1524676146,-1.2441448353,-0.339528651 \backslash$ $C, 0.3595537618,1.2741211585,-0.0300209823 \backslash \mathrm{C}, 0.3595537618,-1.2741211585$ $,-0.0300209823 \backslash \mathrm{C}, 1.0304482284,2.3604104384,-0.8953817336 \backslash \mathrm{C}, 1.030448228$ $4,-2.3604104384,-0.8953817336 \backslash \mathrm{C}, 0.6485026337,1.6141294824,1.4533952721$ $\backslash \mathrm{C}, 0.6485026337,-1.6141294824,1.4533952721 \backslash \mathrm{H},-1.2826615148,1.245157324$ $3,-1.4346576195 \backslash \mathrm{H},-1.2826615148,-1.2451573243,-1.4346576195 \backslash \mathrm{H},-1.64441$ $07135,2.1485680155,0.0459572495 \backslash \mathrm{H},-1.6444107135,-2.1485680155,0.045957$ $2495 \backslash \mathrm{H}, 0.8276583686,2.1700615152,-1.9569931488 \backslash \mathrm{H}, 0.8276583686,-2.17006$ $15152,-1.9569931488 \backslash \mathrm{H}, 0.6778477489,3.3701006228,-0.6382435999 \backslash \mathrm{H}, 0.6778$ $477489,-3.3701006228,-0.6382435999 \backslash \mathrm{H}, 2.1083356234,2.2734365533,-0.7365$ $548848 \backslash \mathrm{H}, 2.1083356234,-2.2734365533,-0.7365548848 \backslash \mathrm{H}, 0.5828919095,2.693$ $3774062,1.6522435541 \backslash \mathrm{H}, 0.5828919095,-2.6933774062,1.6522435541 \backslash \mathrm{H},-0.02$ $60293127,1.1074548126,2.1531347099 \backslash \mathrm{H},-0.0260293127,-1.1074548126,2.153$ $1347099 \backslash \mathrm{H}, 1.6765557744,1.2638578681,1.6144121042 \backslash \mathrm{H}, 1.6765557744,-1.263$ $8578681,1.6144121042 \backslash \backslash$ Version $=I A 32 \mathrm{~L}-\mathrm{G} 03$ RevC $.02 \backslash$ State $=1-A^{\prime} \backslash \mathrm{HF}=-558.9215$ $13 \backslash S 2=0 . \backslash S 2-1=0 . \backslash S 2 A=0 . \backslash R M S D=5.941 e-09 \backslash R M S F=1.141 e-05 \backslash D i p o l e=-2.025719$ $7,0 .,-0.1783184 \backslash \mathrm{PG}=\mathrm{CS} \quad[\mathrm{SG}(\mathrm{C} 1 \mathrm{H} 2 \mathrm{~N} 102), \mathrm{X}(\mathrm{C} 8 \mathrm{H} 16)] \backslash \backslash @$

\section{2,2,6,6-tetraethylpiperidin-1-yloxyl• (7)}

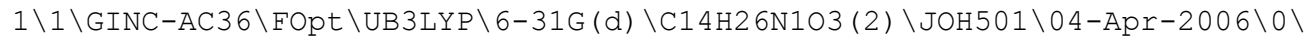
$\backslash \#$ UB3LYP/6-31G* OPT FREQ=noraman maxdisk=671088640 \ctempoeth $26 \backslash \backslash 0,2$ $\backslash N, 1.506184311,0.0014075229,-0.2616849882 \backslash 0,2.7905109397,0.033 \overline{2} 607735$, $-0.3379958667 \backslash \mathrm{C},-1.3091226194,-0.0945091781,0.3012163167 \backslash \mathrm{C},-2.80064878$ $56,-0.1483868913,0.0244630273 \backslash \mathrm{H},-1.1962804136,-0.0052261299,1.38820954$ $04 \backslash C,-0.6979710851,1.1481331496,-0.3748365676 \backslash C,-0.6077479758,-1.34386$ $23496,-0.2306527541 \backslash \mathrm{C}, 0.8110812384,1.3232980736,-0.0938119714 \backslash \mathrm{C}, 0.9140$ $079356,-1.3528382794,0.015374919 \backslash C, 1.4234105998,2.2717865205,-1.158535$ $3539 \backslash C, 1.597037116,-2.3654219748,-0.9434581149 \backslash C, 1.0133120188,1.867134$ $5593,1.3526360913 \backslash \mathrm{C}, 1.2603520264,-1.7146831603,1.4921102483 \backslash \mathrm{H},-1.22466$ $79012,2.0504256763,-0.0508427808 \backslash \mathrm{H},-1.0402928711,-2.2450164916,0.21577$ $87366 \backslash \mathrm{H},-0.8525934526,1.0550316625,-1.4580837678 \backslash \mathrm{H},-0.8175344986,-1.41$ $14775014,-1.3025907655 \backslash \mathrm{H}, 1.2571240775,1.8095078963,-2.140052444 \backslash \mathrm{C}, 1.62$ $38255078,-1.9832018099,-2.4273142221 \backslash \mathrm{C}, 0.8697412849,3.7012820511,-1.17$ $06923122 \backslash \mathrm{H}, 1.0742215615,-3.3223676669,-0.8246140291 \backslash \mathrm{H}, 2.5055916187,2.2$ $921278403,-1.0157065183 \backslash \mathrm{H}, 2.625520444,-2.5186774285,-0.6027667241 \backslash \mathrm{H}, 0$. $4267295975,2.7916225812,1.4326918257 \backslash C, 0.9884535392,-3.1617452515,1.92$ $36840846 \backslash \mathrm{H}, 0.5540607258,1.1672024578,2.0602094392 \backslash \mathrm{H}, 0.7134240903,-1.04$ $10957534,2.1634892475 \backslash \mathrm{C}, 2.4530639131,2.1465486468,1.7973040871 \backslash \mathrm{H}, 2.324$ $4238348,-1.490972669,1.625891461 \backslash 0,-3.3599396774,-0.9022266634,-0.7411$ $713951 \backslash 0,-3.4732667815,0.7922649342,0.7376572987 \backslash \mathrm{H},-4.4107177236,0.706$ $9628728,0.479961938 \backslash \mathrm{H}, 2.4568206683,2.4938376959,2.8372274402 \backslash \mathrm{H}, 3.07809$ $68443,1.2535578035,1.7268872486 \backslash \mathrm{H}, 2.9234186026,2.9253767436,1.18786415$ $\backslash \mathrm{H}, 1.345763096,4.2734542576,-1.97473374 \backslash \mathrm{H},-0.2114563324,3.7319075063,-$ $1.3474767037 \backslash \mathrm{H}, 1.0710225225,4.2314316287,-0.2333685536 \backslash \mathrm{H}, 2.0825723084$, $-2.7909586917,-3.0087657942 \backslash \mathrm{H}, 0.6215041167,-1.8181792228,-2.838624387 \backslash$ $\mathrm{H}, 2.2154310716,-1.0777491005,-2.5848189648 \backslash \mathrm{H}, 1.2273602491,-3.280657611$ $4,2.9866230158 \backslash \mathrm{H},-0.0617845732,-3.4480819307,1.7944151903 \backslash \mathrm{H}, 1.60205883$ $1,-3.8778170983,1.3687934124 \backslash \backslash$ Version=IA64L-G03RevD.01 $\backslash$ State $=2-A \backslash H F=-8$ $29.5286031 \backslash \mathrm{S} 2=0.754061 \backslash \mathrm{S} 2-1=0 . \backslash \mathrm{S} 2 \mathrm{~A}=0.750011 \backslash \mathrm{RMSD}=7.399 \mathrm{e}-09 \backslash \mathrm{RMSF}=1.875 \mathrm{e}$ $-06 \backslash$ Thermal $=0 . \backslash \mathrm{Dipole}=-0.7081192,0.3858622,0.5318177 \backslash \mathrm{PG}=\mathrm{C} 01 \quad[\mathrm{X}(\mathrm{C} 14 \mathrm{H} 26 \mathrm{~N}$ 103) $] \backslash \backslash 0$

\section{2,2,6,6-tetraethylpiperidin-1-yloxyl+}

$1 \backslash 1 \backslash G I N C-A C 35 \backslash F O p t \backslash U B 3 L Y P \backslash 6-31 G(d) \backslash C 14 H 26 N 103(1+) \backslash J O H 501 \backslash 05-A p r-2006 \backslash 0$

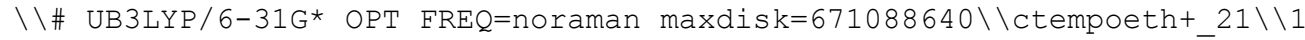
$, 1 \backslash N, 1.5351251017,0.0638609526,0.3919261467 \backslash 0,2.7302692268,0.036 \overline{9} 11059$ 
$1,0.5246547524 \backslash C,-1.4186334185,0.1725469791,0.16322833 \backslash C,-2.4507152421$ $,-0.4200870561,-0.7966958864 \backslash \mathrm{H},-1.9814367027,0.7935806526,0.8678781069$ $\backslash \mathrm{C},-0.4674136264,1.1010170307,-0.6331307446 \backslash \mathrm{C},-0.6845597769,-0.9270436$ $649,0.9605775757 \backslash C, 0.8769290542,1.3919386939,0.0534915332 \backslash C, 0.77210489$ $37,-1.2231387499,0.5571985976 \backslash C, 1.8424370589,2.1669031402,-0.880712860$ $8 \backslash \mathrm{C}, 0.8278870061,-1.9245852154,-0.867328384 \backslash \mathrm{C}, 0.6778082554,2.101443402$ $8,1.4449229851 \backslash \mathrm{C}, 1.4924110397,-2.1161557149,1.6018543884 \backslash \mathrm{H},-0.96348375$ $87,2.0580174005,-0.8072488875 \backslash \mathrm{H},-0.7055008052,-0.6765060303,2.02358217$ $97 \backslash \mathrm{H},-0.2613976229,0.6868108453,-1.6258065421 \backslash \mathrm{H},-1.2270474054,-1.86992$ $09385,0.8480919038 \backslash \mathrm{H}, 1.9991034801,1.5589210181,-1.7807053101 \backslash \mathrm{H}, 0.14728$ $753,-1.4144526822,-1.5508378951 \backslash C, 1.3401504479,3.5575009389,-1.2901623$ $76 \backslash \mathrm{H}, 0.366344276,-2.8984005673,-0.6666784309 \backslash \mathrm{H}, 2.8149134072,2.26042068$ $48,-0.3941469638 \backslash \mathrm{C}, 2.1963651913,-2.1136472481,-1.5191161941 \backslash \mathrm{H}, 0.036337$ $9513,2.9603410222,1.2140166431 \backslash \mathrm{H}, 0.8765536704,-3.0211276703,1.65978724$ $63 \backslash \mathrm{H}, 0.0879267122,1.4540982792,2.1011136178 \backslash \mathrm{C}, 1.6851541951,-1.53523722$ $62,3.007970222 \backslash \mathrm{C}, 1.9381792437,2.5709376111,2.1702158704 \backslash \mathrm{H}, 2.4610392542$ $,-2.4268568471,1.2052177595 \backslash 0,-2.5512093227,-1.5926625761,-1.077195581$ $1 \backslash 0,-3.2273798581,0.548897769,-1.3153614501 \backslash \mathrm{H},-3.8633891483,0.12620723$ $21,-1.9254230728 \backslash \mathrm{H}, 1.6503341261,3.001959843,3.134611161 \backslash \mathrm{H}, 2.6389176241$ $, 1.7552899877,2.3802108972 \backslash \mathrm{H}, 2.4752870239,3.3423064779,1.6122832554 \backslash \mathrm{H}$, $2.093166191,4.0281642752,-1.9291573835 \backslash \mathrm{H}, 0.4084981986,3.5184619586,-1$. $8620425369 \backslash \mathrm{H}, 1.1890653335,4.2160222114,-0.4289981788 \backslash \mathrm{H}, 2.0576241533,-2$ $.6726962454,-2.4506464002 \backslash \mathrm{H}, 2.6800270192,-1.1677332139,-1.7869774367 \backslash \mathrm{H}$ $, 2.8901591048,-2.6876553599,-0.8992805347 \backslash \mathrm{H}, 2.1578057309,-2.2929352327$ $, 3.6400032226 \backslash \mathrm{H}, 2.3463757144,-0.6627525475,3.0118131804 \backslash \mathrm{H}, 0.7415294722$ $,-1.2596146793,3.488943475 \backslash \backslash$ Version=IA64L-G03RevD.01 \State=1-A $\backslash H F=-829$ $.2839384 \backslash \mathrm{S} 2=0 . \backslash \mathrm{S} 2-1=0 . \backslash \mathrm{S} 2 \mathrm{~A}=0 . \backslash \mathrm{RMSD}=5.153 \mathrm{e}-09 \backslash \mathrm{RMSF}=7.648 \mathrm{e}-06 \backslash$ Therma $\mathrm{l}=0$. $\backslash$ Dipole $=0.345866,0.5532041,0.2088015 \backslash \mathrm{PG}=\mathrm{C} 01 \quad[\mathrm{X}(\mathrm{C} 14 \mathrm{H} 26 \mathrm{~N} 1 \mathrm{O} 3)] \backslash \backslash \mathrm{e}$

\section{2,2,6,6-tetraethylpiperidin-1-yloxyl-}

$1 \backslash 1 \backslash G I N C-A C 50 \backslash F O p t \backslash U B 3 L Y P \backslash 6-31 G(d) \backslash C 14 H 26 N 103$ (1-) \JOH501\06-Apr-2006\0 $\backslash \backslash \#$ UB3LYP/6-31G* OPT FREQ=noraman maxdisk=671088640 \\ctempoeth- 56 \\$1,1 \backslash \mathrm{N}, 1.4581788177,0.0497118831,-0.4637775412 \backslash 0,2.8101245646,0.2 \overline{4} 19020$ $368,-0.0986667197 \backslash C,-1.3724629661,-0.1127951749,0.1956732067 \backslash C,-2.8395$ $789271,-0.2239581686,-0.1408668675 \backslash \mathrm{H},-1.3047435594,0.0256503875,1.2800$ $86961 \backslash \mathrm{C},-0.7802492401,1.1351254931,-0.4983863359 \backslash \mathrm{C},-0.5856740218,-1.34$ $79632032,-0.2480704218 \backslash C, 0.7205062102,1.3066785502,-0.1791116802 \backslash C, 0.9$ $329872691,-1.2505666271,0.0440457648 \backslash C, 1.322972708,2.3686985503,-1.152$ $4092043 \backslash \mathrm{C}, 1.5872769091,-2.3658794756,-0.833509216 \backslash \mathrm{C}, 0.9334141173,1.811$ $1046181,1.2823382477 \backslash \mathrm{C}, 1.2484183335,-1.4698143523,1.5519171884 \backslash \mathrm{H},-1.33$ $24413782,2.0349564275,-0.1956618107 \backslash \mathrm{H},-0.9977499147,-2.2508981409,0.22$ $31636333 \backslash \mathrm{H},-0.9175185113,1.014947367,-1.5807295995 \backslash \mathrm{H},-0.738398013,-1.4$ $669025251,-1.3274339875 \backslash \mathrm{C}, 1.6318555951,1.8523523329,-2.5595940732 \backslash \mathrm{H}, 1$. $3504202228,-2.0975611753,-1.8713914387 \backslash \mathrm{H}, 0.6455940497,3.2358895957,-1$. $2005335813 \backslash \mathrm{H}, 1.0708228573,-3.3193196218,-0.6324582893 \backslash \mathrm{H}, 2.2661217581,2$ $.7110749831,-0.7164545119 \backslash \mathrm{C}, 3.1055754144,-2.5583161327,-0.7271531129 \backslash \mathrm{C}$ $, 0.6569381872,3.2922144546,1.578418418 \backslash \mathrm{C}, 1.1112537,-2.8922293016,2.113$ $0904479 \backslash \mathrm{H}, 0.3245765418,1.2177149379,1.9757638543 \backslash \mathrm{H}, 0.6067099531,-0.814$ $1924676,2.1538412919 \backslash \mathrm{H}, 1.9863183089,1.5601879972,1.4710583012 \backslash \mathrm{H}, 2.2717$ $568894,-1.0899212313,1.6638634423 \backslash 0,-3.3437743642,-0.8417005689,-1.058$ $0669674 \backslash 0,-3.6215967988,0.5141154475,0.707881439 \backslash \mathrm{H},-4.5275458904,0.408$ $0956065,0.3620731514 \backslash \mathrm{H}, 0.8006052669,3.5038648331,2.6481962949 \backslash \mathrm{H}, 1.3276$ $422604,3.9584969889,1.0247163822 \backslash \mathrm{H},-0.3735390266,3.5870664976,1.330963$ $8293 \backslash \mathrm{H}, 1.9819113559,2.6772147354,-3.1991141204 \backslash \mathrm{H}, 2.4146432839,1.095943$ $002,-2.4749854506 \backslash \mathrm{H}, 0.7600919617,1.4000023811,-3.0483933204 \backslash \mathrm{H}, 3.451256$ $1471,-3.2817236949,-1.4823742024 \backslash \mathrm{H}, 3.5754778758,-1.5790159932,-0.85613$ $37745 \backslash \mathrm{H}, 3.4059208852,-2.9426257198,0.2561952588 \backslash \mathrm{H}, 1.3281646792,-2.9039$ $639335,3.1913923003 \backslash \mathrm{H}, 0.0959074242,-3.2953165341,1.9881700949 \backslash \mathrm{H}, 1.8008$ $190642,-3.597805065,1.637206719 \backslash \backslash$ Version=IA64L-G03RevD.01 $\backslash$ State=1-A $\backslash H F$ $=-829.5270345 \backslash \mathrm{S} 2=0 . \backslash \mathrm{S} 2-1=0 . \backslash \mathrm{S} 2 \mathrm{~A}=0 . \backslash \mathrm{RMSD}=3.431 \mathrm{e}-09 \backslash \mathrm{RMSF}=3.498 \mathrm{e}-06 \backslash \mathrm{Therm}$ $\mathrm{al}=0 . \backslash \mathrm{Dipole}=-2.4403691,0.1715053,0.6779359 \backslash \mathrm{PG}=\mathrm{C} 01 \quad[\mathrm{X}(\mathrm{C} 14 \mathrm{H} 26 \mathrm{N1O})] \backslash \backslash @$ 


\section{3-carboxy-TEMPO•(8)}

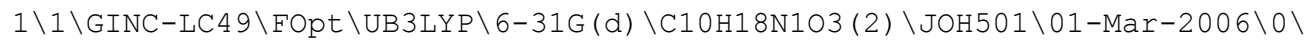

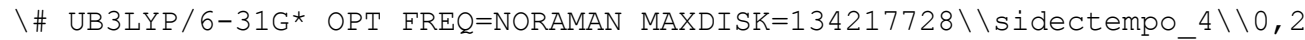
$\backslash N, 1.1793804017,-0.355626939,-0.6184197005 \backslash 0,2.4636606365,-0.37 \overline{2} 357972$ $3,-0.6443450747 \backslash \mathrm{C},-1.6305287098,0.2233271874,-0.5776713552 \backslash \mathrm{H},-2.702556$ $8267,-0.0044490778,-0.5526628345 \backslash \mathrm{H},-1.5526209217,1.3151099229,-0.58221$ $44728 \backslash \mathrm{C},-0.9974485729,-0.3957515352,-1.821638789 \backslash \mathrm{C},-0.9591647172,-0.34$ $74122312,0.6791249202 \backslash C, 0.5122256567,-0.1213684095,-1.9438281534 \backslash C, 0.5$ $765001284,-0.0512343331,0.7309567654 \backslash C, 1.1374341375,-1.107650591,-2.94$ $38062143 \backslash C, 1.260911224,-0.9949516104,1.7353856224 \backslash C, 0.793606799,1.3235$ $074539,-2.4075077024 \backslash \mathrm{C}, 0.8955950116,1.4126923232,1.100104591 \backslash \mathrm{H},-1.4893$ $897223,-0.0243070568,-2.7286301047 \backslash \mathrm{C},-1.6671109937,0.1709878078,1.9209$ $136082 \backslash \mathrm{H},-1.1614106008,-1.4819360952,-1.7997497777 \backslash \mathrm{H},-1.0717931877,-1$. $4382239304,0.6713719214 \backslash \mathrm{H}, 1.0099573039,-2.1395032972,-2.5997150656 \backslash \mathrm{H}, 1$ $.1006541669,-2.0408740824,1.4530187965 \backslash \mathrm{H}, 0.644342004,-1.0040536916,-3$. $9168478388 \backslash \mathrm{H}, 0.8495936562,-0.8428928359,2.7367817908 \backslash \mathrm{H}, 2.2051411354,-0$ $.9162757089,-3.0638109104 \backslash \mathrm{H}, 2.3351477124,-0.8068823325,1.7538788408 \backslash \mathrm{H}$, $0.4664572928,1.4521930219,-3.4454417276 \backslash \mathrm{H}, 0.6731804263,1.6026643089,2$. $154496986 \backslash \mathrm{H}, 0.2729409802,2.0670806038,-1.7967919202 \backslash \mathrm{H}, 0.3220667761,2.1$ $315096271,0.5092968209 \backslash \mathrm{H}, 1.8677246571,1.5215877197,-2.3534505101 \backslash \mathrm{H}, 1.9$ $615085113,1.5873753564,0.9317643147 \backslash 0,-2.1361533069,1.2810387643,2.050$ $2525508 \backslash 0,-1.7418631284,-0.762386602,2.9036677182 \backslash \mathrm{H},-2.2038795671,-0.3$ $319637703,3.6488422821 \backslash \backslash$ Version=IA32L-G03RevC.02 $\backslash$ State $=2-A \backslash H F=-672.281$ $9762 \backslash \mathrm{S} 2=0.753705 \backslash \mathrm{S} 2-1=0 . \backslash \mathrm{S} 2 \mathrm{~A}=0.750009 \backslash \mathrm{RMSD}=4.159 \mathrm{e}-09 \backslash \mathrm{RMSF}=3.491 \mathrm{e}-06 \backslash \mathrm{Di}$ pole $=-0.8422461,-0.2459218,-0.0151603 \backslash P G=C 01 \quad[X(C 10 H 18 N 103)] \backslash \backslash @$

\section{3-carboxy-TEMPO+}

$1 \backslash 1 \backslash G I N C-A C 39 \backslash F O p t \backslash U B 3 L Y P \backslash 6-31 G(d) \backslash C 10 H 18 N 103(1+) \backslash J O H 501 \backslash 09-M a r-2006 \backslash 0$ \\\# UB3LYP/6-31G* OPT FREQ=NORAMAN MAXDISK=134217728\\sidectempo+ $2 \backslash \backslash 1$ $, 1 \backslash \mathrm{N}, 1.1962577674,-0.4430657189,-0.5887751347 \backslash 0,2.243720385,-1.01 \overline{8} 2963$ $499,-0.5941288411 \backslash \mathrm{C},-1.5576689412,0.391700741,-0.5696131646 \backslash \mathrm{H},-2.63564$ $8594,0.2144854411,-0.5620321258 \backslash \mathrm{H},-1.4230036687,1.4790883016,-0.554104$ $8355 \backslash C,-0.9604933016,-0.2306299871,-1.8302335784 \backslash C,-0.9448457679,-0.25$ $36095148,0.6874852292 \backslash C, 0.5723519939,-0.0950181662,-1.9540278229 \backslash C, 0.5$ $991113197,-0.0687218468,0.7926308708 \backslash C, 1.150245287,-1.0690246244,-2.98$ $54177006 \backslash \mathrm{C}, 1.2299041858,-1.0110409367,1.8219727834 \backslash \mathrm{C}, 1.0167293568,1.35$ $39011703,-2.2866486392 \backslash \mathrm{C}, 1.0604410633,1.3922585378,1.0494740904 \backslash \mathrm{H},-1.3$ $883874301,0.2295547681,-2.7272375829 \backslash C,-1.6606822175,0.2057107393,1.96$ $63130234 \backslash \mathrm{H},-1.2218418067,-1.2953980282,-1.8706891837 \backslash \mathrm{H},-1.1397470126,-$ $1.3342476163,0.6264170089 \backslash \mathrm{H}, 0.9831062877,-2.111057633,-2.6964980294 \backslash \mathrm{H}$, $1.0463000686,-2.0617299186,1.5753896767 \backslash \mathrm{H}, 0.6287334021,-0.8911045531,-$ $3.930331624 \backslash \mathrm{H}, 0.7688627982,-0.7884922502,2.7857843406 \backslash \mathrm{H}, 2.2184017063,-$ $0.9152499963,-3.1507566291 \backslash \mathrm{H}, 2.3062545431,-0.854509441,1.9128914633 \backslash \mathrm{H}$, $0.7423954734,1.5283347614,-3.3314649142 \backslash \mathrm{H}, 0.8330568571,1.6061470874,2$. $0947524163 \backslash \mathrm{H}, 0.5208649664,2.1089597342,-1.6768414995 \backslash \mathrm{H}, 0.5396963621,2$. $1233488695,0.4311507411 \backslash \mathrm{H}, 2.1007216437,1.4681589373,-2.1985441766 \backslash \mathrm{H}, 2$. $1386898207,1.4868146923,0.8943500317 \backslash 0,-1.1429310763,0.6925699519,2.94$ $50730704 \backslash 0,-2.9782957644,-0.0296790727,1.8563969389 \backslash \mathrm{H},-3.4027660136,0$. $2584439666,2.6888509701 \backslash \backslash$ Version=IA64L-G03RevC.02 $\backslash$ State $=1-A \backslash H F=-672.02$ $94089 \backslash \mathrm{S} 2=0 . \backslash \mathrm{S} 2-1=0 . \backslash \mathrm{S} 2 \mathrm{~A}=0 . \backslash \mathrm{RMSD}=7.407 \mathrm{e}-09 \backslash \mathrm{RMSF}=4.898 \mathrm{e}-06 \backslash \mathrm{Dipole}=0.1334$ $622,-0.0369757,-1.2938731 \backslash P G=C 01 \quad[X(C 10 H 18 N 103)] \backslash \backslash @$

\section{3-carboxy-TEMPO-}

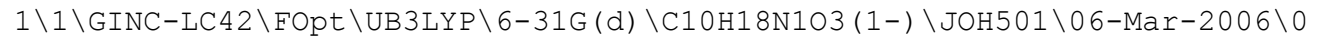
$\backslash \backslash \#$ UB3LYP/6-31G* OPT FREQ=NORAMAN MAXDISK=268435456\\sidectempo- $1 \backslash \backslash-$ $1,1 \backslash \mathrm{N},-1.1264930847,0.7889115241,-0.3892839578 \backslash 0,-1.7902518064,1 . \overline{8} 7652$ $87902,0.1928978542 \backslash \mathrm{C}, 0.3018390513,-1.7463944625,-0.1406303478 \backslash \mathrm{H}, 0.8289$ $061369,-2.6127286596,-0.5668178993 \backslash \mathrm{H}, 0.3222450959,-1.8855783469,0.9453$ $610039 \backslash \mathrm{C},-1.1333888779,-1.6799404257,-0.6605208643 \backslash \mathrm{C}, 1.0447075253,-0.4$ $55167959,-0.5080662821 \backslash \mathrm{C},-1.9037084345,-0.4616306656,-0.1196029028 \backslash \mathrm{C}, 0$ $.3202438636,0.8222433988,0.049794867 \backslash C,-3.2028721412,-0.2838929974,-0$. $9310988323 \backslash C, 0.8689844946,2.0884769777,-0.6383996372 \backslash C,-2.2963212754,-$ 
$0.630463421,1.3674725568 \backslash \mathrm{C}, 0.4844710182,0.9908544295,1.5776442399 \backslash \mathrm{H},-1$ $.6746913415,-2.6041460107,-0.4082802513 \backslash C, 2.4821281355,-0.5377652694,-$ $0.0626829738 \backslash \mathrm{H},-1.1051046804,-1.6167095394,-1.7587133136 \backslash \mathrm{H}, 1.037628836$ $1,-0.3470488637,-1.6010874495 \backslash \mathrm{H},-2.9631407364,-0.1594886676,-1.9945162$ $374 \backslash \mathrm{H}, 0.8340963937,1.9717395733,-1.7286117281 \backslash \mathrm{H},-3.8772827439,-1.14510$ $39181,-0.8145744422 \backslash \mathrm{H}, 1.8975542319,2.3212377209,-0.3392854306 \backslash \mathrm{H},-3.688$ $0446913,0.6319411962,-0.5858068907 \backslash \mathrm{H}, 0.1874863088,2.8961201982,-0.3602$ $910795 \backslash \mathrm{H},-3.1878891574,-1.2624317502,1.4873305302 \backslash \mathrm{H}, 1.4312794748,1.478$ $219448,1.8463990998 \backslash \mathrm{H},-1.4964904243,-1.0660456325,1.9769291548 \backslash \mathrm{H}, 0.435$ $4845993,0.0446295466,2.1255058768 \backslash \mathrm{H},-2.509945398,0.3865699275,1.718231$ $5909 \backslash \mathrm{H},-0.3574607905,1.6346692564,1.8633058416 \backslash 0,2.9235494861,-1.17196$ $25568,0.8806621316 \backslash 0,3.3375884456,0.183217159,-0.8555099506 \backslash \mathrm{H}, 4.207231$ $3191,0.0715890808,-0.4279498937 \backslash \backslash$ Version=IA32L-G03RevC .02 \State=1-A \HF $=-672.2797144 \backslash \mathrm{S} 2=0 . \backslash \mathrm{S} 2-1=0 . \backslash \mathrm{S} 2 \mathrm{~A}=0 . \backslash \mathrm{RMSD}=4.133 \mathrm{e}-09 \backslash \mathrm{RMSF}=9.682 \mathrm{e}-06 \backslash \mathrm{Dipol}$ $\mathrm{e}=1.7527898,-1.5804851,-0.4408496 \backslash \mathrm{PG}=\mathrm{C} 01 \quad[\mathrm{X}(\mathrm{C} 10 \mathrm{H} 18 \mathrm{N1O3})] \backslash \backslash \mathrm{Q}$

\section{3,4-dicarboxy-TEMPO• (9)}

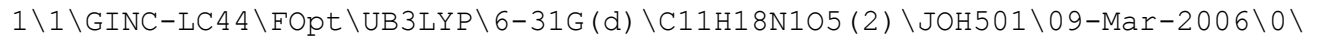

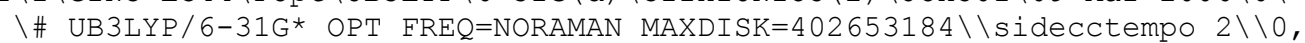
$2 \backslash \mathrm{N}, 1.801515265,-0.3007463064,-0.4949441636 \backslash 0,3.0856077618,-0.28 \overline{5} 00780$ $51,-0.5083746423 \backslash C,-1.0287684224,0.2091695771,-0.4652987918 \backslash C,-2.50750$ $45078,-0.1408722584,-0.4861511313 \backslash \mathrm{H},-0.9610126698,1.3010479657,-0.4706$ $46697 \backslash C,-0.3542652362,-0.3711059116,-1.7202738054 \backslash C,-0.3568107446,-0.3$ $62858093,0.7908895711 \backslash \mathrm{C}, 1.1427711581,-0.0356280065,-1.8149525886 \backslash \mathrm{C}, 1.1$ $765964333,-0.0532335894,0.8562235232 \backslash C, 1.8042304513,-0.9633826226,-2.8$ $47803043 \backslash \mathrm{C}, 1.8656734563,-1.0250711191,1.8307797368 \backslash \mathrm{C}, 1.3788676731,1.43$ $35601101,-2.2226242661 \backslash \mathrm{C}, 1.4740472818,1.4010012833,1.2816437374 \backslash \mathrm{H},-0.8$ $537064953,0.0020377058,-2.6203297993 \backslash C,-1.0762951909,0.1622926566,2.02$ $81291773 \backslash \mathrm{H},-0.4793988069,-1.4619410317,-1.7104308568 \backslash \mathrm{H},-0.4794143495,-$ $1.450589631,0.7808833323 \backslash \mathrm{H}, 1.7087900575,-2.0110624546,-2.5441763169 \backslash \mathrm{H}$, $1.7175374455,-2.0613319196,1.5092265583 \backslash \mathrm{H}, 1.3154273499,-0.8373335787,-$ $3.8201024389 \backslash \mathrm{H}, 1.4484611969,-0.91271283,2.8338130609 \backslash \mathrm{H}, 2.8656875374,-0$ $.7320396736,-2.9499987998 \backslash \mathrm{H}, 2.9375770425,-0.8249723175,1.8578754091 \backslash \mathrm{H}$, $1.0433420625,1.5952392466,-3.2530935976 \backslash \mathrm{H}, 1.2439963835,1.5473928998,2$. $3415560591 \backslash \mathrm{H}, 0.8426849786,2.1383756234,-1.580436635 \backslash \mathrm{H}, 0.8941317966,2.1$ $340355043,0.7146266077 \backslash \mathrm{H}, 2.4473055121,1.6589204668,-2.1640124839 \backslash \mathrm{H}, 2.5$ $381843733,1.5973830574,1.1277262041 \backslash 0,-1.6352034193,1.2361092908,2.098$ $6768773 \backslash 0,-1.0191799365,-0.6955732059,3.0712271585 \backslash \mathrm{H},-1.5093858147,-0$. $268385385,3.7998419708 \backslash 0,-3.0419774642,-0.9980425339,0.1835415762 \backslash 0,-3$ $.1809590787,0.6011297411,-1.3962653321 \backslash \mathrm{H},-4.1083714717,0.2990044417,-1$ $.361530251 \backslash \backslash$ Version=IA32L-G03RevC.02\State $=2-A \backslash H F=-860.8474055 \backslash S 2=0.75$ $3691 \backslash \mathrm{S} 2-1=0 . \backslash \mathrm{S} 2 \mathrm{~A}=0.750009 \backslash \mathrm{RMSD}=7.058 \mathrm{e}-09 \backslash \mathrm{RMSF}=2.955 e-06 \backslash \mathrm{Dipole}=-0.5941$ $715,0.1119977,-0.343216 \backslash P G=C 01 \quad[X(C 11 \mathrm{H} 18 \mathrm{~N} 105)] \backslash \backslash @$

\section{3,4-dicarboxy-TEMPO+}

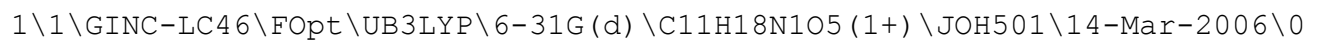
$\backslash \backslash \#$ UB3LYP/6-31G* OPT FREQ=NORAMAN MAXDISK=268435456\\sidecctempo+ $2 \backslash \backslash$ $1,1 \backslash \mathrm{N}, 1.8039998141,-0.507048698,-0.2954419804 \backslash 0,2.7518136775,-1.067272$ $1379,-0.7612957517 \backslash C,-0.6439099239,0.9386747599,0.1826540641 \backslash C,-1.9115$ $665997,1.690801799,-0.2326846816 \backslash \mathrm{H},-0.6300454466,0.9465463506,1.277474$ $5018 \backslash \mathrm{C}, 0.5905495438,1.6581004198,-0.3788441462 \backslash \mathrm{C},-0.7182126855,-0.5057$ $700959,-0.3328395953 \backslash C, 1.9334562203,0.9951406457,-0.0099606113 \backslash C, 0.549$ $1443696,-1.361947489,-0.0021465346 \backslash \mathrm{C}, 3.083039001,1.5353235458,-0.86537$ $0162 \backslash C, 0.6430331323,-2.6053812817,-0.8940058982 \backslash C, 2.2769406765,1.13799$ $18127,1.4958176159 \backslash \mathrm{C}, 0.6630192852,-1.7702115555,1.4931482187 \backslash \mathrm{H}, 0.62921$ $49187,2.6856745554,-0.0062165795 \backslash C,-1.9586222813,-1.1923211513,0.26344$ $12684 \backslash \mathrm{H}, 0.5167519577,1.7185547072,-1.4719682049 \backslash \mathrm{H},-0.8396776779,-0.490$ $3072813,-1.4203739031 \backslash \mathrm{H}, 2.9336835388,1.32106965,-1.9278311294 \backslash \mathrm{H}, 0.7478$ $952456,-2.3375906179,-1.9496904657 \backslash \mathrm{H}, 3.1048641087,2.6212975569,-0.7369$ $579009 \backslash \mathrm{H},-0.2871460603,-3.1658185625,-0.7815849334 \backslash \mathrm{H}, 4.0515730648,1.13$ $60806327,-0.5575897975 \backslash \mathrm{H}, 1.4755827663,-3.2504274348,-0.6074177052 \backslash \mathrm{H}, 2$. $5362216041,2.1889769048,1.6551160301 \backslash \mathrm{H},-0.0534860237,-2.5783876559,1.6$ $601220654 \backslash \mathrm{H}, 1.4445884822,0.891994239,2.1546235082 \backslash \mathrm{H}, 0.4153505585,-0.96$ 
$5586171,2.1841636274 \backslash \mathrm{H}, 3.1458100493,0.5303582046,1.7634960666 \backslash \mathrm{H}, 1.6621$ $774821,-2.1568203288,1.711672637 \backslash 0,-2.3556398521,-0.9777042817,1.38479$ $18956 \backslash 0,-2.5072060503,-2.0692411045,-0.5893516593 \backslash \mathrm{H},-3.3046050479,-2.4$ $430201239,-0.163372688 \backslash 0,-2.7833874613,1.2269678113,-0.9290050002 \backslash 0,-1$ $.914938899,2.9375445846,0.2681061993 \backslash \mathrm{H},-2.7431079678,3.3719788299,-0.0$ $167939621 \backslash \backslash$ Version=IA32L-G03RevC.02 \State $=1-A \backslash H F=-860.5902363 \backslash S 2=0 . \backslash S 2$ $-1=0 . \backslash \mathrm{S} 2 \mathrm{~A}=0 . \backslash \mathrm{RMSD}=3.967 \mathrm{e}-09 \backslash \mathrm{RMSF}=8.931 \mathrm{e}-06 \backslash \mathrm{Dipole}=1.976584,0.0906643,0$ $.0582788 \backslash \mathrm{PG}=\mathrm{C} 01 \quad[\mathrm{X}(\mathrm{C} 11 \mathrm{H} 18 \mathrm{~N} 105)] \backslash \backslash \mathrm{Q}$

\section{3,4-dicarboxy-TEMPO-}

$1 \backslash 1 \backslash G I N C-L C 105 \backslash F O p t \backslash U B 3 L Y P \backslash 6-31 G(d) \backslash C 11 H 18 N 105$ (1-) \JOH501\15-Mar-2006 $0 \backslash \backslash \#$ UB3LYP/6-31G* OPT FREQ=NORAMAN MAXDISK=268435456\\sidecctempo- 2\ $\backslash-1,1 \backslash \mathrm{N}, 1.7877570014,-0.5229081463,-0.4817076345 \backslash 0,2.9402739688,-1 . \overline{2} 02$ $2737891,-0.067911101 \backslash \mathrm{C},-0.6556026842,0.9463082948,0.1545485037 \backslash \mathrm{C},-1.88$ $78848392,1.7370011905,-0.2185590618 \backslash \mathrm{H},-0.5973734065,0.9422300786,1.246$ $051656 \backslash C, 0.5908053392,1.6479010457,-0.4232403225 \backslash C,-0.719759033,-0.494$ $0779017,-0.3737145724 \backslash \mathrm{C}, 1.8899808817,0.906437934,-0.0630761262 \backslash \mathrm{C}, 0.577$ $9891537,-1.3019393466,-0.0179644335 \backslash C, 3.035209856,1.4778291795,-0.9238$ $611818 \backslash \mathrm{C}, 0.6486016891,-2.5894831783,-0.8647485003 \backslash \mathrm{C}, 2.2789434368,1.088$ $3087408,1.422887957 \backslash \mathrm{C}, 0.6514254254,-1.6977979706,1.4749924628 \backslash \mathrm{H}, 0.6365$ $961158,2.6842061116,-0.0631507272 \backslash \mathrm{C},-1.9712415204,-1.1644537843,0.1407$ $404671 \backslash \mathrm{H}, 0.4891000854,1.6806764628,-1.5166398154 \backslash \mathrm{H},-0.7811285388,-0.45$ $80738285,-1.4671781334 \backslash \mathrm{H}, 2.7924575541,1.3686991353,-1.9879028248 \backslash \mathrm{H}, 0.5$ $108897807,-2.3521925452,-1.9266059779 \backslash \mathrm{H}, 3.2211011697,2.5391260919,-0.7$ $043277405 \backslash \mathrm{H},-0.0966766539,-3.3350835843,-0.5676233756 \backslash \mathrm{H}, 3.9270496947,0$ $.8813292929,-0.7187309011 \backslash \mathrm{H}, 1.6631199509,-2.9722724873,-0.7286630532 \backslash \mathrm{H}$ $, 2.763944601,2.057259302,1.6057011424 \backslash \mathrm{H}, 0.0751834329,-2.6059677148,1.6$ $962702194 \backslash \mathrm{H}, 1.4261243479,1.0101839464,2.1065926172 \backslash \mathrm{H}, 0.3013085789,-0.9$ $120376128,2.1525181406 \backslash \mathrm{H}, 2.9834677092,0.27255398,1.6258748087 \backslash \mathrm{H}, 1.7195$ $886684,-1.886559827,1.6434795452 \backslash 0,-2.5897956693,-0.8620043206,1.14767$ $48173 \backslash 0,-2.4014068293,-2.1920399632,-0.6459367994 \backslash \mathrm{H},-3.1887416688,-2.5$ $354765996,-0.1839193789 \backslash 0,-2.6250949166,1.5428162423,-1.1652768803 \backslash 0,-$ $2.0885622525,2.8003072694,0.6138467127 \backslash \mathrm{H},-2.8744310713,3.2511080884,0$. $2530020928 \backslash \backslash$ Version $=I A 32 L-G 03 R e v C .02 \backslash$ State $=1-A \backslash H F=-860.8516621 \backslash S 2=0 . \backslash S$ $2-1=0 . \backslash S 2 A=0 . \backslash R M S D=8.927 e-09 \backslash R M S F=1.093 e-05 \backslash D i p o l e=-2.6309414,1.256716$ $4,0.1549075 \backslash P G=C 01 \quad[X(C 11 \mathrm{H} 18 \mathrm{~N} 105)] \backslash \backslash @$

\section{3,5-dicarboxy-TEMPO•(10)}

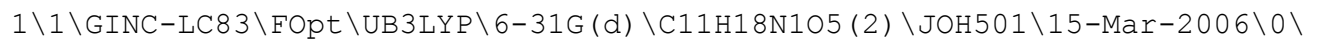
\\# UB3LYP/6-31G* OPT FREQ=NORAMAN MAXDISK=268435456\\oppcctempo_2s $\backslash \backslash 0$, $2 \backslash \mathrm{N}, 1.5174257229,-0.3403220046,0 . \backslash 0,2.8012107438,-0.3404748815, \overline{0} . \backslash \mathrm{C},-1$ $.2987356854,0.1554326902,0 . \backslash \mathrm{H},-1.2625264472,1.2470884118,0 . \backslash \mathrm{H},-2.36227$ $24555,-0.1085248937,0 . \backslash 0,-1.8882754998,1.1477115899,2.6292477624 \backslash 0,-1$. $8882754998,1.1477115899,-2.6292477624 \backslash \mathrm{C},-1.3848865103,0.0547487588,2.4$ $898462145 \backslash C,-1.3848865103,0.0547487588,-2.4898462145 \backslash 0,-1.4522966033,-$ $0.9012858142,3.4504581973 \backslash 0,-1.4522966033,-0.9012858142,-3.4504581973 \backslash$ $\mathrm{H},-1.9439380158,-0.5019249432,4.1940056849 \backslash \mathrm{H},-1.9439380158,-0.50192494$ $32,-4.1940056849 \backslash C,-0.6316657863,-0.4178394937,1.2552101991 \backslash C,-0.63166$ $57863,-0.4178394937,-1.2552101991 \backslash \mathrm{H},-0.7043018192,-1.5116489359,1.2287$ $687486 \backslash \mathrm{H},-0.7043018192,-1.5116489359,-1.2287687486 \backslash \mathrm{C}, 0.8873690552,-0.0$ $650635656,1.3419236281 \backslash \mathrm{C}, 0.8873690552,-0.0650635656,-1.3419236281 \backslash \mathrm{C}, 1$. $1460021395,1.4063077139,1.7284799555 \backslash C, 1.1460021395,1.4063077139,-1.72$ $84799555 \backslash C, 1.5876309733,-0.9917462993,2.3507844277 \backslash C, 1.5876309733,-0.9$ $917462993,-2.3507844277 \backslash \mathrm{H}, 0.5532490107,2.1087022792,1.1377759446 \backslash \mathrm{H}, 0.5$ $532490107,2.1087022792,-1.1377759446 \backslash \mathrm{H}, 0.9032176377,1.5759046475,2.781$ $6849354 \backslash \mathrm{H}, 0.9032176377,1.5759046475,-2.7816849354 \backslash \mathrm{H}, 2.2067042871,1.623$ $5263334,1.5771166893 \backslash \mathrm{H}, 2.2067042871,1.6235263334,-1.5771166893 \backslash \mathrm{H}, 1.145$ $0143113,-0.8676567601,3.34255214 \backslash \mathrm{H}, 1.1450143113,-0.8676567601,-3.34255$ $214 \backslash \mathrm{H}, 1.4787337312,-2.0401821085,2.0542466155 \backslash \mathrm{H}, 1.4787337312,-2.040182$ $1085,-2.0542466155 \backslash \mathrm{H}, 2.6519719551,-0.7591226901,2.3995129957 \backslash \mathrm{H}, 2.65197$ $19551,-0.7591226901,-2.3995129957 \backslash \backslash$ Version=IA32L-G03RevC.02 \State=2-A ' $\backslash \mathrm{HF}=-860.8430788 \backslash \mathrm{S} 2=0.753705 \backslash \mathrm{S} 2-1=0 . \backslash \mathrm{S} 2 \mathrm{~A}=0.750009 \backslash \mathrm{RMSD}=7.659 \mathrm{e}-09 \backslash \mathrm{RMSF}=$ $2.318 e-06 \backslash$ Dipole $=-0.5701541,-0.6840996,0 . \backslash P G=C S \quad[S G(C 1 H 2 N 1 O 1), X(C 10 H 16$ 
04) $] \backslash \backslash @$

\section{3,5-dicarboxy-TEMPO+}

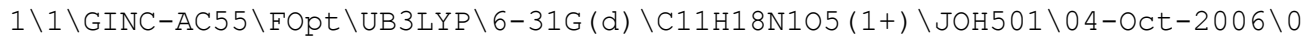
\\\# UB3LYP/6-31G* OPT FREQ=noraman maxdisk=536870912\\oppcctempo+_5s \\ $1,1 \backslash \mathrm{N},-1.6089401657,-0.1199221131,0 . \backslash 0,-2.7972548407,-0.001564975 \overline{9}, 0 . \backslash$ $\mathrm{C}, 1.2447937635,0.1468945135,0 . \backslash \mathrm{H}, 2.1763330526,0.7131028505,0 . \backslash \mathrm{H}, 1.5202$ $354951,-0.9137343583,0 . \backslash C, 0.4472743221,0.5120270957,-1.2637210079 \backslash \mathrm{C}, 0$. $4472743221,0.5120270957,1.2637210079 \backslash \mathrm{H}, 0.230687062,1.5892949674,-1.210$ $5339899 \backslash \mathrm{H}, 0.230687062,1.5892949674,1.2105339899 \backslash 0,2.4570318596,1.01230$ $74662,-2.3924666347 \backslash 0,2.4570318596,1.0123074662,2.3924666347 \backslash \mathrm{H}, 2.96213$ $1842,0.9102776193,-3.2235733624 \backslash \mathrm{H}, 2.962131842,0.9102776193,3.223573362$ $4 \backslash \mathrm{C}, 1.297704829,0.3500547857,-2.5342352067 \backslash \mathrm{C}, 1.297704829,0.3500547857$, $2.5342352067 \backslash 0,0.9844210602,-0.2541809696,-3.5338070093 \backslash 0,0.9844210602$ $,-0.2541809696,3.5338070093 \backslash \mathrm{C},-0.915224718,-0.2272003382,-1.3830104999$ $\backslash \mathrm{C},-0.915224718,-0.2272003382,1.3830104999 \backslash \mathrm{C},-0.8044939584,-1.75039682$ $43,-1.6673757811 \backslash \mathrm{C},-0.8044939584,-1.7503968243,1.6673757811 \backslash \mathrm{C},-1.84896$ $87287,0.4334097747,-2.4025416779 \backslash C,-1.8489687287,0.4334097747,2.402541$ $6779 \backslash \mathrm{H},-2.0701434908,1.471847997,-2.135736805 \backslash \mathrm{H},-2.0701434908,1.471847$ $997,2.135736805 \backslash \mathrm{H},-1.3352247596,0.4180894795,-3.3652918727 \backslash \mathrm{H},-1.335224$ $7596,0.4180894795,3.3652918727 \backslash \mathrm{H},-2.7893880444,-0.1104961922,-2.506666$ $2709 \backslash \mathrm{H},-2.7893880444,-0.1104961922,2.5066662709 \backslash \mathrm{H},-0.0518563923,-2.250$ $5085214,-1.0578094555 \backslash \mathrm{H},-0.0518563923,-2.2505085214,1.0578094555 \backslash \mathrm{H},-0$. $5139436004,-1.8442672809,-2.7145602761 \backslash \mathrm{H},-0.5139436004,-1.8442672809,2$ $.7145602761 \backslash \mathrm{H},-1.7722559347,-2.2377920171,-1.5220204563 \backslash \mathrm{H},-1.772255934$ $7,-2.2377920171,1.5220204563 \backslash \backslash$ Version=IA64L-G03RevD.01 $\backslash$ State=1-A ' $\backslash H F=-$ $860.5874568 \backslash \mathrm{S} 2=0 . \backslash \mathrm{S} 2-1=0 . \backslash \mathrm{S} 2 \mathrm{~A}=0 . \backslash \mathrm{RMSD}=2.481 \mathrm{e}-09 \backslash \mathrm{RMSF}=4.559 \mathrm{e}-06 \backslash \mathrm{Therma} 1$ $=0 . \backslash$ Dipole $=-0.2696797,0.1784954,0 . \backslash P G=C S \quad[S G(C 1 H 2 N 101), X(C 10 H 1604)] \backslash \backslash @$

\section{3,5-dicarboxy-TEMPO-}

$1 \backslash 1 \backslash G I N C-L C 61 \backslash F O p t \backslash U B 3 L Y P \backslash 6-31 G(d) \backslash C 11 H 18 N 105(1-) \backslash J O H 501 \backslash 17-M a r-2006 \backslash 0$ $\backslash \backslash \#$ UB3LYP/6-31G* OPT FREQ=NORAMAN MAXDISK=268435456\\oppcctempo- $2 \mathrm{~s} \backslash \backslash$ $-1,1 \backslash \mathrm{N}, 1.4956446147,-0.5563540262,0 . \backslash 0,2.8358052209,-0.1689986924,0 . \backslash \mathrm{C}$ $,-1.3323733321,0.1379020018,0 . \backslash \mathrm{H},-1.2889223875,1.2292552523,0 . \backslash \mathrm{H},-2.40$ $04993608,-0.1187110468,0 . \backslash 0,-1.9895608475,1.0962740209,2.6226034356 \backslash 0$, $-1.9895608475,1.0962740209,-2.6226034356 \backslash \mathrm{C},-1.3848874383,0.0478098682$, $2.486660804 \backslash \mathrm{C},-1.3848874383,0.0478098682,-2.486660804 \backslash 0,-1.3335890177$, $-0.8508024875,3.5196383715 \backslash 0,-1.3335890177,-0.8508024875,-3.5196383715$ $\backslash \mathrm{H},-1.800407288,-0.4026830632,4.2496316313 \backslash \mathrm{H},-1.800407288,-0.402683063$ $2,-4.2496316313 \backslash C,-0.6577224853,-0.4272678757,1.2534886462 \backslash C,-0.657722$ $4853,-0.4272678757,-1.2534886462 \backslash \mathrm{H},-0.7229484758,-1.5230221548,1.22925$ $21669 \backslash \mathrm{H},-0.7229484758,-1.5230221548,-1.2292521669 \backslash \mathrm{C}, 0.8719667362,-0.08$ $89738898,1.2959065137 \backslash \mathrm{C}, 0.8719667362,-0.0889738898,-1.2959065137 \backslash \mathrm{C}, 1.1$ $512084899,1.3968726002,1.6106022395 \backslash C, 1.1512084899,1.3968726002,-1.610$ $6022395 \backslash \mathrm{C}, 1.5777297961,-0.9518568166,2.3611501142 \backslash \mathrm{C}, 1.5777297961,-0.95$ $18568166,-2.3611501142 \backslash \mathrm{H}, 0.4540607381,2.081274508,1.1180418153 \backslash \mathrm{H}, 0.454$ $0607381,2.081274508,-1.1180418153 \backslash \mathrm{H}, 1.1138142539,1.6056423367,2.687710$ $6043 \backslash \mathrm{H}, 1.1138142539,1.6056423367,-2.6877106043 \backslash \mathrm{H}, 2.1678095303,1.567878$ $7798,1.2368531187 \backslash \mathrm{H}, 2.1678095303,1.5678787798,-1.2368531187 \backslash \mathrm{H}, 1.288308$ $7874,-0.677188576,3.3819985556 \backslash \mathrm{H}, 1.2883087874,-0.677188576,-3.38199855$ $56 \backslash \mathrm{H}, 1.3489653714,-2.012481128,2.2010313935 \backslash \mathrm{H}, 1.3489653714,-2.01248112$ $8,-2.2010313935 \backslash \mathrm{H}, 2.6496792489,-0.8084405338,2.2090569715 \backslash \mathrm{H}, 2.64967924$ $89,-0.8084405338,-2.2090569715 \backslash \backslash$ Version=IA32L-G03RevC.02 $\backslash$ State=1-A' $\backslash H F$ $=-860.8503244 \backslash \mathrm{S} 2=0 . \backslash \mathrm{S} 2-1=0 . \backslash \mathrm{S} 2 \mathrm{~A}=0 . \backslash \mathrm{RMSD}=4.602 \mathrm{e}-09 \backslash \mathrm{RMSF}=2.070 \mathrm{e}-05 \backslash \mathrm{Dipol}$ $\mathrm{e}=-2.2800283,-0.5166408,0 . \backslash \mathrm{PG}=\mathrm{CS} \quad[\mathrm{SG}(\mathrm{C} 1 \mathrm{H} 2 \mathrm{~N} 101), \mathrm{X}(\mathrm{C} 10 \mathrm{H} 1604)] \backslash \backslash @$

\section{4-NH ${ }_{3}{ }^{+}$-TEMPO• (11)}

$1 \backslash 1 \backslash G I N C-L C 7 \backslash F O p t \backslash U B 3 L Y P \backslash 6-31 G(d) \backslash C 9 H 20 N 2 O 1(1+, 2) \backslash J O H 501 \backslash 13-M a r-2006 \backslash 0$

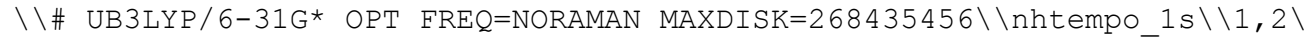
$0,2.3430208045,-0.2874769503,0 . \backslash N, 1.0613442067,-0.2851428718, \overline{0} . \backslash \mathrm{C},-1.7$ $301287997,0.2373023285,0 . \backslash \mathrm{H},-1.7169873146,1.330232757,0 . \backslash \mathrm{H},-3.36041393$ 
$68,-1.1380664407,0 . \backslash \mathrm{N},-3.231235941,-0.118106133,0 . \backslash \mathrm{H},-3.708714465,0.25$ $30383041,0.8309657447 \backslash \mathrm{H},-3.708714465,0.2530383041,-0.8309657447 \backslash \mathrm{C},-1.0$ $883724517,-0.3388400613,1.2546262555 \backslash \mathrm{C},-1.0883724517,-0.3388400613,-1$. $2546262555 \backslash C, 0.423255296,-0.0093354894,1.3315959 \backslash C, 0.423255296,-0.0093$ $354894,-1.3315959 \backslash \mathrm{C}, 0.6689305266,1.4562052817,1.7474864263 \backslash \mathrm{C}, 0.6689305$ $266,1.4562052817,-1.7474864263 \backslash C, 1.0777296947,-0.9481762873,2.35829346$ $65 \backslash \mathrm{C}, 1.0777296947,-0.9481762873,-2.3582934665 \backslash \mathrm{H}, 0.1412146612,2.1802431$ $39,1.1181055905 \backslash \mathrm{H}, 0.1412146612,2.180243139,-1.1181055905 \backslash \mathrm{H}, 0.352418253$ $4,1.6132273914,2.7841345347 \backslash \mathrm{H}, 0.3524182534,1.6132273914,-2.7841345347 \backslash$ $\mathrm{H}, 1.7387373582,1.6689062894,1.6793359634 \backslash \mathrm{H}, 1.7387373582,1.6689062894,-$ $1.6793359634 \backslash \mathrm{H}, 0.6100368986,-0.8125363936,3.3391147047 \backslash \mathrm{H}, 0.6100368986$, $-0.8125363936,-3.3391147047 \backslash \mathrm{H}, 2.142367359,-0.727395189,2.4424921766 \backslash \mathrm{H}$, $2.142367359,-0.727395189,-2.4424921766 \backslash \mathrm{H}, 0.969266626,-1.9949813465,2.0$ $57123877 \backslash \mathrm{H}, 0.969266626,-1.9949813465,-2.057123877 \backslash \mathrm{H},-1.5698394599,0.04$ $43956498,2.1630700801 \backslash \mathrm{H},-1.5698394599,0.0443956498,-2.1630700801 \backslash \mathrm{H},-1$. $2131207484,-1.4307293317,1.2451023615 \backslash \mathrm{H},-1.2131207484,-1.4307293317,-1$ $.2451023615 \backslash \backslash$ Version=IA32L-G03RevC.02\State $=2-A^{\prime} \backslash H F=-539.429651 \backslash S 2=0.7$ $53583 \backslash \mathrm{S} 2-1=0 . \backslash \mathrm{S} 2 \mathrm{~A}=0.750009 \backslash \mathrm{RMSD}=6.171 \mathrm{e}-09 \backslash \mathrm{RMSF}=7.318 \mathrm{e}-06 \backslash \mathrm{Dipole}=-5.321$ $498,0.0636527,0 . \backslash \mathrm{PG}=\mathrm{CS} \quad[\mathrm{SG}(\mathrm{C} 1 \mathrm{H} 2 \mathrm{~N} 2 \mathrm{O} 1), \mathrm{X}(\mathrm{C} 8 \mathrm{H} 18)] \backslash \backslash \mathrm{C}$

\section{4- $\mathrm{NH}_{3}{ }^{+}$-TEMPO+}

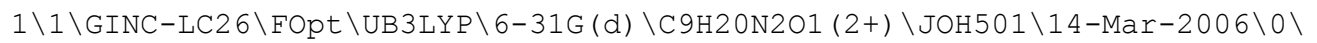

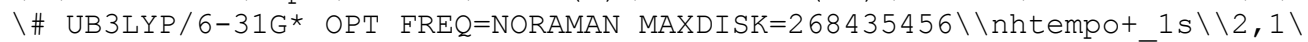
$0,2.2513170168,-0.5129780165,0 . \backslash \mathrm{N}, 1.0967647239,-0.2209299776, \overline{0} . \backslash \mathrm{C},-1.7$ $46936151,0.2450125793,0 . \backslash \mathrm{H},-1.7362679193,1.338186428,0 . \backslash \mathrm{H},-3.393911215$ $9,-1.13228569,0 . \backslash \mathrm{N},-3.238380805,-0.113638587,0 . \backslash \mathrm{H},-3.7220200388,0.2652$ $70092,0.8273998138 \backslash \mathrm{H},-3.7220200388,0.265270092,-0.8273998138 \backslash \mathrm{C},-1.0898$ $481003,-0.3279713108,1.2574939371 \backslash \mathrm{C},-1.0898481003,-0.3279713108,-1.257$ $4939371 \backslash \mathrm{C}, 0.4191384961,0.0024202309,1.3735571579 \backslash \mathrm{C}, 0.4191384961,0.0024$ $202309,-1.3735571579 \backslash C, 0.6908940517,1.4870976687,1.7383827132 \backslash C, 0.6908$ $940517,1.4870976687,-1.7383827132 \backslash \mathrm{C}, 1.1025604222,-0.9278332696,2.38580$ $11092 \backslash \mathrm{C}, 1.1025604222,-0.9278332696,-2.3858011092 \backslash \mathrm{H}, 0.1701836934,2.2036$ $263598,1.0999463034 \backslash \mathrm{H}, 0.1701836934,2.2036263598,-1.0999463034 \backslash \mathrm{H}, 0.3457$ $914227,1.6294744964,2.7675092075 \backslash \mathrm{H}, 0.3457914227,1.6294744964,-2.767509$ $2075 \backslash \mathrm{H}, 1.7626870372,1.7002672898,1.7135766695 \backslash \mathrm{H}, 1.7626870372,1.7002672$ $898,-1.7135766695 \backslash \mathrm{H}, 0.5779302481,-0.8276210215,3.3409040976 \backslash \mathrm{H}, 0.577930$ $2481,-0.8276210215,-3.3409040976 \backslash \mathrm{H}, 2.1463820694,-0.6531788627,2.546571$ $7249 \backslash \mathrm{H}, 2.1463820694,-0.6531788627,-2.5465717249 \backslash \mathrm{H}, 1.0527972085,-1.9760$ $385037,2.0753275643 \backslash \mathrm{H}, 1.0527972085,-1.9760385037,-2.0753275643 \backslash \mathrm{H},-1.55$ $98450955,0.0598539544,2.1686396705 \backslash \mathrm{H},-1.5598450955,0.0598539544,-2.168$ $6396705 \backslash \mathrm{H},-1.2140895251,-1.4190197845,1.2679309059 \backslash \mathrm{H},-1.2140895251,-1$. $4190197845,-1.2679309059 \backslash \backslash$ Version=IA32L-G03RevC.02 $\backslash$ State $=1-A^{\prime} \backslash H F=-539$. $0534155 \backslash S 2=0 . \backslash S 2-1=0 . \backslash S 2 A=0 . \backslash R M S D=2.126 e-09 \backslash R M S F=2.401 e-05 \backslash D i p o l e=-4.0$ $063691,0.0556127,0 . \backslash P G=C S \quad[S G(C 1 H 2 N 2 O 1), X(C 8 H 18)] \backslash \backslash @$

\section{4- $\mathrm{NH}_{3}{ }^{+}$-TEMPO-}

$1 \backslash 1 \backslash G I N C-L C 6 \backslash F O p t \backslash U B 3 L Y P \backslash 6-31 G(d) \backslash C 9 H 20 N 201 \backslash J O H 501 \backslash 13-M a r-2006 \backslash 0 \backslash \backslash \#$ UB

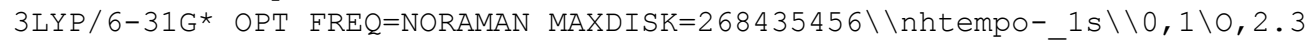
$741475549,-0.1917098411,0 . \backslash \mathrm{N}, 1.0155623078,-0.4704037225, \overline{0} . \backslash \mathrm{C},-1.730499$ $741,0.2157084979,0 . \backslash \mathrm{H},-1.7163492912,1.3085348218,0 . \backslash \mathrm{H},-3.3595143946,-1$ $.1540125493,0 . \backslash \mathrm{N},-3.2454795895,-0.1294404241,0 . \backslash \mathrm{H},-3.7187890106,0.2424$ $433091,0.8371634239 \backslash \mathrm{H},-3.7187890106,0.2424433091,-0.8371634239 \backslash \mathrm{C},-1.10$ $43280769,-0.3476387555,1.2625567302 \backslash C,-1.1043280769,-0.3476387555,-1.2$ $625567302 \backslash C, 0.411020106,-0.0246336464,1.2813022921 \backslash C, 0.411020106,-0.02$ $46336464,-1.2813022921 \backslash \mathrm{C}, 0.6932516078,1.4574856387,1.6173481546 \backslash \mathrm{C}, 0.69$ $32516078,1.4574856387,-1.6173481546 \backslash \mathrm{C}, 1.0750820402,-0.8926630852,2.367$ $8033791 \backslash \mathrm{C}, 1.0750820402,-0.8926630852,-2.3678033791 \backslash \mathrm{H}, 0.0338700813,2.16$ $74808962,1.103651728 \backslash \mathrm{H}, 0.0338700813,2.1674808962,-1.103651728 \backslash \mathrm{H}, 0.6257$ $69905,1.6584106851,2.693751792 \backslash \mathrm{H}, 0.625769905,1.6584106851,-2.693751792$ $\backslash \mathrm{H}, 1.7235588779,1.6191303837,1.2766524693 \backslash \mathrm{H}, 1.7235588779,1.6191303837$, $-1.2766524693 \backslash \mathrm{H}, 0.6881574588,-0.6564693201,3.367911344 \backslash \mathrm{H}, 0.6881574588$, $-0.6564693201,-3.367911344 \backslash \mathrm{H}, 2.1496779748,-0.7098482924,2.3213428252 \backslash \mathrm{H}$ $, 2.1496779748,-0.7098482924,-2.3213428252 \backslash \mathrm{H}, 0.9065316252,-1.9547962969$ 
$, 2.1556184245 \backslash \mathrm{H}, 0.9065316252,-1.9547962969,-2.1556184245 \backslash \mathrm{H},-1.59025549$ $01,0.0593905791,2.161334736 \backslash \mathrm{H},-1.5902554901,0.0593905791,-2.161334736 \backslash$ $\mathrm{H},-1.231124152,-1.4391356061,1.267672777 \backslash \mathrm{H},-1.231124152,-1.4391356061$, $-1.267672777 \backslash \backslash$ Version=IA32L-G03RevC.02 $\backslash$ State $=1-A^{\prime} \backslash H F=-539.5444735 \backslash \mathrm{S} 2=0$ $. \backslash S 2-1=0 . \backslash S 2 A=0 . \backslash R M S D=7.772 e-09 \backslash R M S F=1.314 e-05 \backslash D i p o l e=-6.1579629,0.130$ $7253,0 . \backslash P G=C S \quad[S G(C 1 H 2 N 2 O 1), X(C 8 H 18)] \backslash \backslash @$

\section{4-oxo-TEMPO•(12)}

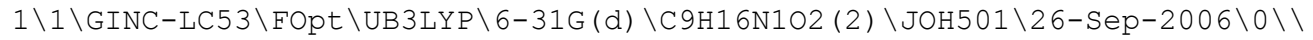
\# UB3LYP/6-31G* OPT FREQ=NORAMAN MAXDISK=402653184 \\otempo $3 \mathrm{~s} \backslash \backslash 0,2 \backslash \mathrm{N}, 0$ $.9191072282,-0.3663536961,0 . \backslash 0,2.2036919777,-0.3554966109, \overline{0} . \backslash \mathrm{C},-1.8771$ $63973,0.1075003976,0 . \backslash 0,-2.7869609694,0.9140447034,0 . \backslash C,-1.238218444,-$ $0.4377863931,-1.2616135577 \backslash C,-1.238218444,-0.4377863931,1.2616135577 \backslash \mathrm{C}$ $, 0.2732036846,-0.1045607091,-1.3331420765 \backslash C, 0.2732036846,-0.1045607091$ $, 1.3331420765 \backslash \mathrm{C}, 0.9564934451,-1.0226443169,-2.3581479987 \backslash \mathrm{C}, 0.956493445$ $1,-1.0226443169,2.3581479987 \backslash C, 0.486285866,1.3704718068,-1.7319311862 \backslash$ $\mathrm{C}, 0.486285866,1.3704718068,1.7319311862 \backslash \mathrm{H},-1.3622911742,-1.5293167601$, $-1.2644646317 \backslash \mathrm{H},-1.3622911742,-1.5293167601,1.2644646317 \backslash \mathrm{H},-1.74867896$ $25,-0.0311649256,-2.1388613365 \backslash \mathrm{H},-1.7486789625,-0.0311649256,2.1388613$ $365 \backslash \mathrm{H}, 0.8766253955,-2.0714105388,-2.0533469245 \backslash \mathrm{H}, 0.8766253955,-2.07141$ $05388,2.0533469245 \backslash \mathrm{H}, 0.472074045,-0.9056109101,-3.333485527 \backslash \mathrm{H}, 0.472074$ $045,-0.9056109101,3.333485527 \backslash \mathrm{H}, 2.0146527878,-0.7731650885,-2.45311276$ $28 \backslash \mathrm{H}, 2.0146527878,-0.7731650885,2.4531127628 \backslash \mathrm{H}, 0.1984926951,1.51851312$ $49,-2.7786470704 \backslash \mathrm{H}, 0.1984926951,1.5185131249,2.7786470704 \backslash \mathrm{H},-0.1126400$ $363,2.0548595931,-1.1238805812 \backslash \mathrm{H},-0.1126400363,2.0548595931,1.12388058$ $12 \backslash \mathrm{H}, 1.5428705262,1.6299575527,-1.6211005038 \backslash \mathrm{H}, 1.5428705262,1.62995755$ $27,1.6211005038 \backslash \backslash$ Version=IA32L-G03RevC.02 State $^{2}=2-\mathrm{A}^{\prime} \backslash \mathrm{HF}=-557.7286681 \backslash \mathrm{S}$ $2=0.753651 \backslash \mathrm{S} 2-1=0 . \backslash \mathrm{S} 2 \mathrm{~A}=0.750009 \backslash \mathrm{RMSD}=4.249 \mathrm{e}-09 \backslash \mathrm{RMSF}=1.013 \mathrm{e}-05 \backslash \mathrm{Dipole}=-$ $0.1051982,-0.4465692,0 . \backslash P G=C S \quad[S G(C 1 N 1 O 2), X(C 8 H 16)] \backslash \backslash @$

\section{4-OXO-TEMPO+}

$1 \backslash 1 \backslash G I N C-A C 27 \backslash F O p t \backslash U B 3 L Y P \backslash 6-31 G(d) \backslash C 9 H 16 N 102(1+) \backslash J O H 501 \backslash 03-O c t-2006 \backslash 0 \backslash$

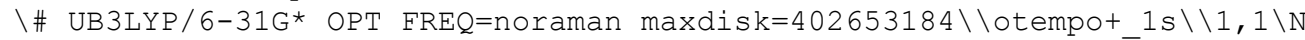
$,-0.9875952131,-0.1474217319,0 . \backslash 0,-2.1766938934,-0.258028273 \overline{3}, 0 . \backslash \mathrm{C}, 1.8$ $470197272,-0.3220946354,0 . \backslash 0,2.8971673684,0.2755407653,0 . \backslash \mathrm{C}, 1.10883710$ $96,-0.7206671566,1.2702834382 \backslash C, 1.1088371096,-0.7206671566,-1.27028343$ $82 \backslash \mathrm{C},-0.2822204215,-0.0515129677,1.3750686818 \backslash \mathrm{C},-0.2822204215,-0.05151$ $29677,-1.3750686818 \backslash C,-1.1800567046,-0.7445478389,2.4033700848 \backslash C,-1.18$ $00567046,-0.7445478389,-2.4033700848 \backslash C,-0.1626129132,1.4644111384,1.68$ $64217814 \backslash \mathrm{C},-0.1626129132,1.4644111384,-1.6864217814 \backslash \mathrm{H}, 0.9911973416,-1$. $8118372937,1.2931467069 \backslash \mathrm{H}, 0.9911973416,-1.8118372937,-1.2931467069 \backslash \mathrm{H}, 1$ $.6946879687,-0.4230984811,2.1436220168 \backslash \mathrm{H}, 1.6946879687,-0.4230984811,-2$ $.1436220168 \backslash \mathrm{H},-1.396820728,-1.7790610754,2.1201615666 \backslash \mathrm{H},-1.396820728,-$ $1.7790610754,-2.1201615666 \backslash \mathrm{H},-0.6368086503,-0.7589837918,3.352601257 \backslash \mathrm{H}$ $,-0.6368086503,-0.7589837918,-3.352601257 \backslash \mathrm{H},-2.1210347826,-0.212505370$ $9,2.5563675218 \backslash \mathrm{H},-2.1210347826,-0.2125053709,-2.5563675218 \backslash \mathrm{H}, 0.1472805$ $195,1.5396286748,2.7332477989 \backslash \mathrm{H}, 0.1472805195,1.5396286748,-2.733247798$ $9 \backslash \mathrm{H}, 0.5921102903,1.9657770051,1.0790940528 \backslash \mathrm{H}, 0.5921102903,1.9657770051$ $,-1.0790940528 \backslash \mathrm{H},-1.1257495244,1.9709555954,1.5807299073 \backslash \mathrm{H},-1.12574952$ $44,1.9709555954,-1.5807299073 \backslash \backslash$ Version=IA64L-G03RevD.01 $\backslash$ State=1 $-A^{\prime} \backslash H F=$ $-557.4653558 \backslash \mathrm{S} 2=0 . \backslash \mathrm{S} 2-1=0 . \backslash \mathrm{S} 2 \mathrm{~A}=0 . \backslash \mathrm{RMSD}=6.191 \mathrm{e}-09 \backslash \mathrm{RMSF}=7.511 \mathrm{e}-06 \backslash \mathrm{Therma}$ $l=0 . \backslash$ Dipole $=-1.1333652,-0.2655428,0 . \backslash P G=C S \quad[S G(C 1 N 1 O 2), X(C 8 H 16)] \backslash \backslash @$

\section{4-0X0-TEMPO-}

$1 \backslash 1 \backslash G I N C-A C 29 \backslash F O p t \backslash U B 3 L Y P \backslash 6-31 G(d) \backslash C 9 H 16 N 1 O 2(1-) \backslash J O H 501 \backslash 03-O c t-2006 \backslash 0 \backslash$

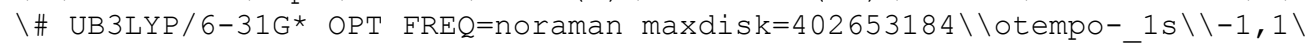
$\mathrm{N},-0.9979866338,-0.3649097363,0 . \backslash 0,-2.235864089,0.3061132251,0 . \backslash \mathrm{C}, 1.88$ $26821124,-0.3806669739,0 . \backslash 0,2.9819297766,0.159070541,0 . \backslash \mathrm{C}, 1.125254342$, $-0.7331821246,1.2596607824 \backslash \mathrm{C}, 1.125254342,-0.7331821246,-1.2596607824 \backslash \mathrm{C}$ $,-0.2799819574,-0.0692642842,1.2758941126 \backslash C,-0.2799819574,-0.069264284$ $2,-1.2758941126 \backslash C,-1.1388463414,-0.7354895624,2.368121644 \backslash C,-1.1388463$ 
$414,-0.7354895624,-2.368121644 \backslash C,-0.1815669535,1.4405106967,1.59573505$ $83 \backslash \mathrm{C},-0.1815669535,1.4405106967,-1.5957350583 \backslash \mathrm{H}, 0.9853421512,-1.823876$ $8659,1.2705206127 \backslash \mathrm{H}, 0.9853421512,-1.8238768659,-1.2705206127 \backslash \mathrm{H}, 1.71450$ $4606,-0.4460110461,2.1380052027 \backslash \mathrm{H}, 1.714504606,-0.4460110461,-2.1380052$ $027 \backslash \mathrm{H},-1.2090490024,-1.814588073,2.1819538417 \backslash \mathrm{H},-1.2090490024,-1.81458$ $8073,-2.1819538417 \backslash \mathrm{H},-0.7251892155,-0.5699378079,3.3732474649 \backslash \mathrm{H},-0.725$ $1892155,-0.5699378079,-3.3732474649 \backslash \mathrm{H},-2.1429951165,-0.3124997375,2.28$ $63381993 \backslash \mathrm{H},-2.1429951165,-0.3124997375,-2.2863381993 \backslash \mathrm{H},-0.0705896877,1$ $.6271101512,2.673261868 \backslash \mathrm{H},-0.0705896877,1.6271101512,-2.673261868 \backslash \mathrm{H}, 0$. $6574790286,1.9290054181,1.0883101967 \backslash \mathrm{H}, 0.6574790286,1.9290054181,-1.08$ $83101967 \backslash \mathrm{H},-1.1309839369,1.860976208,1.2410795355 \backslash \mathrm{H},-1.1309839369,1.86$ $0976208,-1.2410795355 \backslash \backslash$ Version=IA64L-G03RevD.01 \State=1-A ' \HF=-557.730 $3659 \backslash \mathrm{S} 2=0 . \backslash \mathrm{S} 2-1=0 . \backslash \mathrm{S} 2 \mathrm{~A}=0 . \backslash \mathrm{RMSD}=4.399 \mathrm{e}-09 \backslash \mathrm{RMSF}=1.515 \mathrm{e}-05 \backslash \mathrm{Thermal}=0 . \backslash \mathrm{Dip}$ ole $=1.2778709,-0.6135952,0 . \backslash \mathrm{PG}=\mathrm{CS} \quad[\mathrm{SG}(\mathrm{C} 1 \mathrm{~N} 1 \mathrm{O} 2), \mathrm{X}(\mathrm{C} 8 \mathrm{H} 16)] \backslash \backslash @$

\section{TEMPENE• (13)}

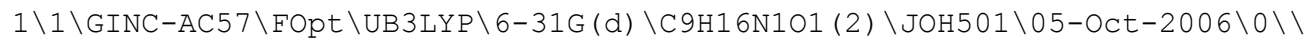

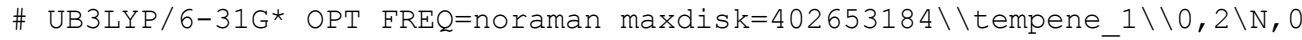
$.776893002,0.1151140043,0.0159710256 \backslash 0,2.0515832819,-0.0425 \overline{5} 02705,0.00$ $85932248 \backslash \mathrm{C},-2.0134392602,0.4955514738,-0.0321976849 \backslash \mathrm{C},-1.2588327505,0$. $8004477392,1.2321646567 \backslash C,-1.4052217911,0.0879877348,-1.1459283411 \backslash C, 0$ $.0784386285,0.0365703442,1.3358794892 \backslash \mathrm{C}, 0.0852659961,-0.0936531797,-1$. $2935158575 \backslash C, 0.9796219556,0.6982457181,2.3865875671 \backslash C, 0.640016752,0.96$ $54186412,-2.2719465892 \backslash \mathrm{C},-0.1562735936,-1.4440798185,1.7037756044 \backslash \mathrm{C}, 0$. $411861696,-1.5079778086,-1.8235344915 \backslash \mathrm{H},-1.8687520896,0.5539034365,2.1$ $106714564 \backslash \mathrm{H},-1.0658085156,1.881976629,1.2945420612 \backslash \mathrm{H}, 1.2255332135,1.72$ $49739166,2.0972395867 \backslash \mathrm{H}, 0.4004520142,1.9747008955,-1.9228967868 \backslash \mathrm{H}, 0.45$ $39104163,0.7237327809,3.3477703991 \backslash \mathrm{H}, 0.2021450837,0.8287589199,-3.2665$ $689775 \backslash \mathrm{H}, 1.9136177283,0.1472789913,2.5072548587 \backslash \mathrm{H}, 1.7260410749,0.86591$ $44501,-2.3431212977 \backslash \mathrm{H},-0.5312587157,-1.5226551176,2.7309275638 \backslash \mathrm{H},-0.01$ $3740499,-1.6427036477,-2.8241837983 \backslash \mathrm{H},-0.8878816879,-1.9135008936,1.03$ $91460711 \backslash \mathrm{H},-0.0005283382,-2.2828802584,-1.170398406 \backslash \mathrm{H}, 0.7866349353,-1$. $995622012,1.6371481392 \backslash \mathrm{H}, 1.4958560835,-1.6356873021,-1.8793281775 \backslash \mathrm{H},-3$ $.093174983,0.6332537648,-0.0248158902 \backslash \mathrm{H},-1.9820796374,-0.1135891316,-2$ $.0472354058 \backslash \backslash$ Version=IA64L-G03RevD.01 \State $=2-A \backslash H F=-482.4898936 \backslash S 2=0.7$ $53721 \backslash \mathrm{S} 2-1=0 . \backslash \mathrm{S} 2 \mathrm{~A}=0.750009 \backslash \mathrm{RMSD}=7.279 \mathrm{e}-09 \backslash \mathrm{RMSF}=1.452 \mathrm{e}-05 \backslash \mathrm{Thermal}=0 . \backslash \mathrm{Di}$ pole $=-1.0755966,-0.0053067,0.0575144 \backslash \mathrm{PG}=\mathrm{C01}[\mathrm{X}(\mathrm{C} 9 \mathrm{H} 16 \mathrm{~N} 1 \mathrm{O} 1)] \backslash \backslash @$

\section{TEMPENE+}

$1 \backslash 1 \backslash G I N C-A C 47 \backslash F O p t \backslash U B 3 L Y P \backslash 6-31 G(d) \backslash C 9 H 16 N 1 O 1(1+) \backslash J O H 501 \backslash 05-O c t-2006 \backslash 0 \backslash$

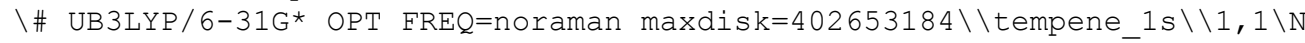
$, 0.7920158481,0.0694219753,0.0259143995 \backslash 0,1.9802440133,0.201 \overline{1} 098819,0$. $0021289343 \backslash \mathrm{C},-2.0066505386,0.4893022212,-0.028067933 \backslash \mathrm{C},-1.2290510747,0$ $.8670977069,1.2013068937 \backslash \mathrm{C},-1.4199029559,0.0126467295,-1.1246158022 \backslash \mathrm{C}$, $0.0789441674,0.0587123795,1.3686377677 \backslash C, 0.0697564963,-0.1247499288,-1$ $.313407989 \backslash C, 1.0087701293,0.6561951333,2.4224110804 \backslash C, 0.6107987706,0.9$ $705337016,-2.2630269967 \backslash C,-0.2088289098,-1.4412747442,1.6598491109 \backslash \mathrm{C}, 0$ $.4800543568,-1.5322676233,-1.8194878877 \backslash \mathrm{H},-1.8204970565,0.6827777001,2$ $.1051174391 \backslash \mathrm{H},-1.0045700637,1.943215817,1.2042627599 \backslash \mathrm{H}, 1.3226951112,1$. $6701142747,2.156232696 \backslash \mathrm{H}, 0.4005474777,1.9711806889,-1.8753414909 \backslash \mathrm{H}, 0.4$ $544223358,0.7072702603,3.363863057 \backslash \mathrm{H}, 0.0905769624,0.8628808837,-3.2186$ $09897 \backslash \mathrm{H}, 1.8986904185,0.0441497156,2.5842910749 \backslash \mathrm{H}, 1.6833449604,0.856822$ $4161,-2.4348445859 \backslash \mathrm{H},-0.6352816563,-1.4851342412,2.6668833717 \backslash \mathrm{H}, 0.0253$ $864768,-1.6675019042,-2.8050504281 \backslash \mathrm{H},-0.9292556635,-1.872653948,0.9634$ $275084 \backslash \mathrm{H}, 0.1165207364,-2.3276785342,-1.1649969616 \backslash \mathrm{H}, 0.7141012372,-2.02$ $76982708,1.6551855813 \backslash \mathrm{H}, 1.5656523355,-1.6048325533,-1.9251221157 \backslash \mathrm{H},-3$. $0852701146,0.6161638411,-0.0031629798 \backslash \mathrm{H},-2.0023337999,-0.2568735786,-2$ $.0017766073 \backslash \backslash$ Version=IA64L-G03RevD.01 \State=1-A \HF=-482.2388382 \S2=0. $\mathrm{S} 2-1=0 . \backslash \mathrm{S} 2 \mathrm{~A}=0 . \backslash \mathrm{RMSD}=6.302 \mathrm{e}-09 \backslash \mathrm{RMSF}=1.721 \mathrm{e}-05 \backslash \mathrm{Thermal}=0 . \backslash \mathrm{Dipole}=-0.3001$ $203,-0.1150083,0.0991915 \backslash \mathrm{PG}=\mathrm{CO} 1 \quad[\mathrm{X}(\mathrm{C} 9 \mathrm{H} 16 \mathrm{~N} 1 \mathrm{O} 1)] \backslash \backslash \mathrm{e}$ 


\section{TEMPENE-}

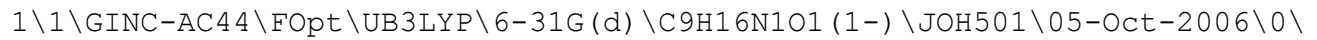

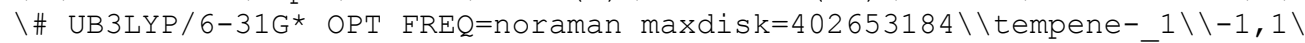
$\mathrm{N}, 0.7722510765,0.3793654176,0.0037140644 \backslash 0,2.0641800861,-0.1781024629$, $0.0104118648 \backslash C,-2.0540802397,0.5668782407,-0.0327474124 \backslash C,-1.321815830$ $4,0.7414542446,1.2704893708 \backslash \mathrm{C},-1.4258759093,0.2284898679,-1.1610763992$ $\backslash C, 0.070157207,0.0675043638,1.2787509442 \backslash C, 0.0560100354,-0.0275911407$, $-1.2493272104 \backslash \mathrm{C}, 0.9340743349,0.7029605002,2.3839599065 \backslash \mathrm{C}, 0.676680832,0$ $.8806115554,-2.337649686 \backslash \mathrm{C},-0.0314666874,-1.4506920536,1.5625151106 \backslash \mathrm{C}$, $0.3283600853,-1.50488265,-1.6433409431 \backslash \mathrm{H},-1.9275333137,0.3471443021,2$. $1032894102 \backslash \mathrm{H},-1.2039103892,1.8188267082,1.4718358568 \backslash \mathrm{H}, 1.0344090392,1$. $7806876866,2.2031692426 \backslash \mathrm{H}, 0.4591200113,1.9319488371,-2.1164982123 \backslash \mathrm{H}, 0$. $4939676621,0.5474753129,3.380674359 \backslash \mathrm{H}, 0.3110878661,0.6395011203,-3.347$ $0872261 \backslash \mathrm{H}, 1.9303655922,0.260062863,2.3251316378 \backslash \mathrm{H}, 1.7592439037,0.73181$ $96705,-2.2800331272 \backslash \mathrm{H},-0.1889679959,-1.6689664565,2.6296190392 \backslash \mathrm{H}, 0.275$ $7956698,-1.663175757,-2.7296086816 \backslash \mathrm{H},-0.8469737233,-1.9209531409,1.000$ $5885391 \backslash \mathrm{H},-0.3686595591,-2.2018863279,-1.1652889815 \backslash \mathrm{H}, 0.9296080756,-1$. $8702732112,1.2415582993 \backslash \mathrm{H}, 1.3489398429,-1.6790389684,-1.2696489041 \backslash \mathrm{H},-$ $3.1318875995,0.7492444257,-0.0398932584 \backslash \mathrm{H},-1.9922000727,0.1305170525,-$ $2.0915076029 \backslash \backslash$ Version=IA64L-G03RevD.01 \State $=1-A \backslash H F=-482.4771135 \backslash S 2=0$. $\backslash \mathrm{S} 2-1=0 . \backslash \mathrm{S} 2 \mathrm{~A}=0 . \backslash \mathrm{RMSD}=3.025 \mathrm{e}-09 \backslash \mathrm{RMSF}=1.605 \mathrm{e}-05 \backslash \mathrm{Thermal}=0 . \backslash \mathrm{Dipole}=-1.972$ $9693,0.1027274,0.055156 \backslash \mathrm{PG}=\mathrm{C} 01 \quad[\mathrm{X}(\mathrm{C} 9 \mathrm{H} 16 \mathrm{~N} 101)] \backslash \backslash @$

\section{PROXYL・(14)}

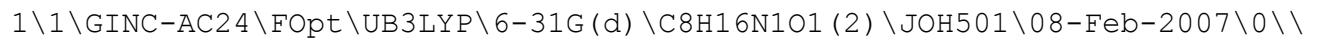

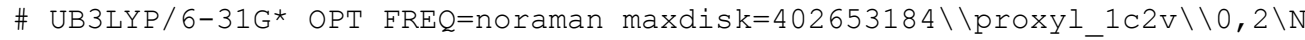
$, 0 ., 0 .,-0.0015115311 \backslash 0,0 ., 0 ., 1.2744073363 \backslash C, 1.2599403567,0.0001224556$, $-0.7968992085 \backslash C,-1.2599403567,-0.0001224556,-0.7968992085 \backslash C, 0.72038645$ $29,-0.2760312376,-2.2164817156 \backslash C,-0.7203864529,0.2760312376,-2.2164817$ $156 \backslash \mathrm{H}, 1.3431566966,0.1855603524,-2.989328976 \backslash \mathrm{H},-1.3431566966,-0.185560$ $3524,-2.989328976 \backslash \mathrm{H}, 0.7124168473,-1.3560796869,-2.4049191929 \backslash \mathrm{H},-0.7124$ $168473,1.3560796869,-2.4049191929 \backslash \mathrm{C}, 1.9248445118,1.3838144722,-0.67090$ $3006 \backslash C,-1.9248445118,-1.3838144722,-0.670903006 \backslash C, 2.1967095165,-1.0911$ $653876,-0.2686669487 \backslash \mathrm{C},-2.1967095165,1.0911653876,-0.2686669487 \backslash \mathrm{H}, 1.29$ $88224567,2.171099688,-1.1052610945 \backslash \mathrm{H},-1.2988224567,-2.171099688,-1.105$ $2610945 \backslash \mathrm{H}, 2.8939705963,1.3942338745,-1.1826742603 \backslash \mathrm{H},-2.8939705963,-1.3$ $942338745,-1.1826742603 \backslash \mathrm{H}, 2.0837389957,1.6191744517,0.3860982878 \backslash \mathrm{H},-2$. $0837389957,-1.6191744517,0.3860982878 \backslash \mathrm{H}, 1.7308083454,-2.0791274147,-0$. $3495400638 \backslash \mathrm{H},-1.7308083454,2.0791274147,-0.3495400638 \backslash \mathrm{H}, 3.1323786875,-$ $1.1009334462,-0.8388186756 \backslash \mathrm{H},-3.1323786875,1.1009334462,-0.8388186756 \backslash$ $\mathrm{H}, 2.4269928996,-0.9090076418,0.784862575 \backslash \mathrm{H},-2.4269928996,0.9090076418$, $0.784862575 \backslash \backslash$ Version=IA64L-G03RevD.01 \State $=2-B \backslash H F=-444.4105462 \backslash S 2=0.7$ $53779 \backslash \mathrm{S} 2-1=0 . \backslash \mathrm{S} 2 \mathrm{~A}=0.75001 \backslash \mathrm{RMSD}=7.253 \mathrm{e}-09 \backslash \mathrm{RMSF}=2.947 \mathrm{e}-05 \backslash$ Thermal=0 . $\backslash \mathrm{Dip}$ $\mathrm{ole}=0 ., 0 .,-1.2008263 \backslash \mathrm{PG}=\mathrm{C} 02 \quad[\mathrm{C} 2(\mathrm{~N} 1 \mathrm{O} 1), \mathrm{X}(\mathrm{C} 8 \mathrm{H} 16)] \backslash \backslash @$

\section{PROXYL+}

$1 \backslash 1 \backslash G I N C-A C 21 \backslash F O p t \backslash U B 3 L Y P \backslash 6-31 G(d) \backslash C 8 H 16 N 101(1+) \backslash J O H 501 \backslash 19-F e b-2007 \backslash 0 \backslash$

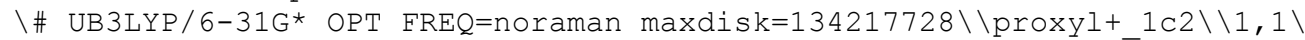
$\mathrm{N}, 0 ., 0.0 .6792086375 \backslash 0,0 ., 0 ., 1.8707914633 \backslash \mathrm{C}, 0.0403093513,1.2 \overline{7} 50506138$, $-0.1469533855 \backslash C,-0.0403093513,-1.2750506138,-0.1469533855 \backslash C, 0.30443993$ $28,0.7089249919,-1.5544588856 \backslash C,-0.3044399328,-0.7089249919,-1.5544588$ $856 \backslash \mathrm{H},-0.1445138635,1.3471231549,-2.318848104 \backslash \mathrm{H}, 0.1445138635,-1.347123$ $1549,-2.318848104 \backslash \mathrm{H}, 1.3816399095,0.6743749285,-1.7472589809 \backslash \mathrm{H},-1.38163$ $99095,-0.6743749285,-1.7472589809 \backslash \mathrm{C},-1.3806879113,1.8829143324,0.00028$ $36872 \backslash C, 1.3806879113,-1.8829143324,0.0002836872 \backslash C, 1.1106181739,2.21050$ $11628,0.4211659995 \backslash \mathrm{C},-1.1106181739,-2.2105011628,0.4211659995 \backslash \mathrm{H},-2.161$ $8511407,1.2241140186,-0.3889754544 \backslash \mathrm{H}, 2.1618511407,-1.2241140186,-0.388$ $9754544 \backslash \mathrm{H},-1.3950638899,2.8102393751,-0.5797072182 \backslash \mathrm{H}, 1.3950638899,-2.8$ $102393751,-0.5797072182 \backslash \mathrm{H},-1.59348554,2.1175414607,1.0466521293 \backslash \mathrm{H}, 1.59$ $348554,-2.1175414607,1.0466521293 \backslash \mathrm{H}, 2.1028485214,1.7503219838,0.389046$ $1151 \backslash \mathrm{H},-2.1028485214,-1.7503219838,0.3890461151 \backslash \mathrm{H}, 1.1339130431,3.11267$ $39198,-0.1971428675 \backslash \mathrm{H},-1.1339130431,-3.1126739198,-0.1971428675 \backslash \mathrm{H}, 0.88$ 
$24709396,2.5036100719,1.4490184146 \backslash \mathrm{H},-0.8824709396,-2.5036100719,1.449$ $0184146 \backslash \backslash$ Version=IA64L-G03RevD.01 \State $=1-A \backslash H F=-444.1617704 \backslash \mathrm{S} 2=0 . \backslash \mathrm{S} 2-1$ $=0 . \backslash \mathrm{S} 2 \mathrm{~A}=0 . \backslash \mathrm{RMSD}=8.386 \mathrm{e}-09 \backslash \mathrm{RMSF}=4.410 \mathrm{e}-05 \backslash \mathrm{Thermal}=0 . \backslash \mathrm{Dipole}=0,, 0 .,-0.48$ $28517 \backslash \mathrm{PG}=\mathrm{C} 02 \quad[\mathrm{C} 2(\mathrm{~N} 1 \mathrm{O} 1), \mathrm{X}(\mathrm{C} 8 \mathrm{H} 16)] \backslash \backslash @$

\section{PROXYL-}

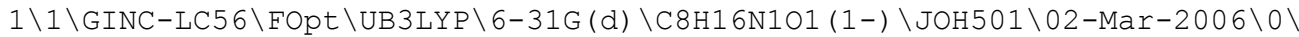
\\# UB3LYP/6-31G* OPT FREQ=NORAMAN MAXDISK=134217728\\proxyl- $1 \mathrm{C} \backslash \backslash-1,1 \backslash$ $\mathrm{N}, 0.0172319316,-0.5308275972,-0.5652065426 \backslash \mathrm{C}, 1.2120828,0.165653944,-0$. $0179148893 \backslash \mathrm{C}, 0.7658001609,1.634130275,-0.1090821895 \backslash \mathrm{C},-1.2085704922,0$. $1404176053,-0.0032741533 \backslash \mathrm{C},-0.7375077559,1.5961035881,0.2706364759 \backslash \mathrm{H},-$ $0.868739745,1.8422422177,1.3321225392 \backslash \mathrm{H},-1.3155550652,2.3418931897,-0$. $2936168888 \backslash \mathrm{C},-2.2673222859,0.0482588701,-1.1169606967 \backslash \mathrm{C}, 2.3791466542,-$ $0.1902276426,-0.9474274841 \backslash C,-1.7436986085,-0.5902067461,1.2476098534 \backslash$ $\mathrm{C}, 1.5558500779,-0.2959021503,1.4176154736 \backslash \mathrm{H}, 3.3368354126,0.2353819433$, $-0.6126091469 \backslash \mathrm{H}, 2.4438613826,-1.2839324587,-0.9728137435 \backslash \mathrm{H}, 2.168589002$ $, 0.1566883018,-1.9670056623 \backslash 0,0.0467259822,-1.9084610594,-0.3841190009$ $\backslash \mathrm{H}, 2.6155117339,-0.1538900275,1.6759285799 \backslash \mathrm{H}, 0.9548706823,0.2186407878$ $, 2.177852444 \backslash \mathrm{H}, 1.3052464263,-1.3670461105,1.4235359743 \backslash \mathrm{H},-2.377229085$, $-1.0100610295,-1.3813433674 \backslash \mathrm{H},-3.2457800355,0.4514735743,-0.8117901665$ $\backslash \mathrm{H},-1.9230637033,0.5828662354,-2.0108970255 \backslash \mathrm{H},-1.465270331,-1.64188866$ $51,1.0770784097 \backslash \mathrm{H},-1.2646432515,-0.2462196329,2.172562572 \backslash \mathrm{H},-2.8310629$ $186,-0.4845214399,1.3704902677 \backslash \mathrm{H}, 0.891507626,1.9895372523,-1.139874900$ $7 \backslash \mathrm{H}, 1.3458071881,2.3029510559,0.5425635792 \backslash \backslash$ Version=IA32L-G03RevC.02 \S tate $=1-\mathrm{A} \backslash \mathrm{HF}=-444.3907129 \backslash \mathrm{S} 2=0 . \backslash \mathrm{S} 2-1=0 . \backslash \mathrm{S} 2 \mathrm{~A}=0 . \backslash \mathrm{RMSD}=2.797 e-09 \backslash \mathrm{RMSF}=7.01$ $4 e-06 \backslash$ Dipole $=-0.0142267,1.9201228,0.4584936 \backslash P G=C 01 \quad[X(C 8 H 16 N 101)] \backslash \backslash @$

\section{3-carboxy-PROXYL・ (15)}

$1 \backslash 1 \backslash G I N C-L C 45 \backslash F O p t \backslash U B 3 L Y P \backslash 6-31 G(d) \backslash C 9 H 16 N 103(2) \backslash J O H 501 \backslash 24-F e b-2006 \backslash 0 \backslash \backslash$

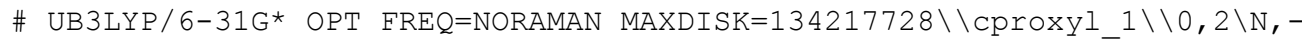
$0.9386281158,-0.7922993282,-0.6499716908 \backslash C, 0.3818744661,-0 . \overline{9} 189196302$, $0.0258225899 \backslash \mathrm{C}, 0.8393884235,0.5747315301,0.0321812775 \backslash \mathrm{C},-1.4773656507$, $0.5904186514,-0.7775393784 \backslash \mathrm{C},-0.4654722188,1.3934514186,0.0625540515 \backslash \mathrm{H}$ $,-0.8062947697,1.4893900322,1.0975924319 \backslash \mathrm{H},-0.3172805527,2.4054351206$, $-0.3265682978 \backslash \mathrm{C},-1.4681646273,0.9866878746,-2.265740366 \backslash \mathrm{C}, 1.2956542074$ $,-1.828799074,-0.8008424269 \backslash \mathrm{C},-2.9051509541,0.6366405061,-0.2212885597$ $\backslash \mathrm{C}, 0.1624665074,-1.509441505,1.4317835962 \backslash \mathrm{H}, 2.2775833561,-1.9138401819$ $,-0.3256098783 \backslash \mathrm{H}, 0.84793899,-2.8228965029,-0.8837312867 \backslash \mathrm{H}, 1.4315906585$ $,-1.4308919172,-1.8121757308 \backslash 0,-1.593566478,-1.8167833363,-1.033762672$ $2 \backslash \mathrm{H}, 1.1197974374,-1.7218062761,1.9200829593 \backslash \mathrm{H},-0.4085390663,-0.8322989$ $023,2.0744475847 \backslash \mathrm{H},-0.3939782702,-2.4461220569,1.3333292499 \backslash \mathrm{H},-2.03628$ $20151,0.2524807607,-2.8452260146 \backslash \mathrm{H},-1.9247653892,1.9731743461,-2.40432$ $24998 \backslash \mathrm{H},-0.4483932238,1.0213948112,-2.6653733894 \backslash \mathrm{H},-3.5430327448,-0.06$ $16474711,-0.7704436889 \backslash \mathrm{H},-2.9217941474,0.3562098939,0.8371510971 \backslash \mathrm{H},-3$. $3194431685,1.6461338655,-0.3184254153 \backslash \mathrm{C}, 1.7711440209,0.9124690316,1.17$ $68369876 \backslash 0,1.4658158149,1.4775593766,2.2036699333 \backslash \mathrm{H}, 1.385572139,0.7572$ $303,-0.89998134 \backslash 0,3.0356787459,0.4813859246,0.9339595089 \backslash \mathrm{H}, 3.558047869$ $3,0.7134209369,1.7255152645 \backslash \backslash$ Version=IA32L-G03RevC.02 $\backslash$ State $=2-A \backslash H F=-63$ $2.9754585 \backslash \mathrm{S} 2=0.753776 \backslash \mathrm{S} 2-1=0 . \backslash \mathrm{S} 2 \mathrm{~A}=0.75001 \backslash \mathrm{RMSD}=8.928 \mathrm{e}-09 \backslash \mathrm{RMSF}=4.993 e-0$ $6 \backslash$ Dipole $=0.7485791,0.6098897,-0.0282996 \backslash P G=C 01 \quad[X(C 9 H 16 \mathrm{~N} 103)] \backslash \backslash @$

\section{3-carboxy-PROXYL+}

$1 \backslash 1 \backslash G I N C-L C 32 \backslash F O p t \backslash U B 3 L Y P \backslash 6-31 G(d) \backslash C 9 H 16 N 103(1+) \backslash J O H 501 \backslash 09-M a r-2006 \backslash 0 \backslash$

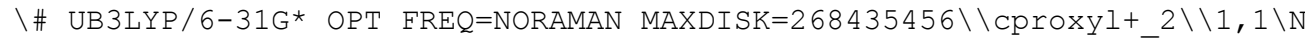
$,-1.0517405936,-0.8874821989,-0.3679292269 \backslash \mathrm{C}, 0.4622147074,-0 . \overline{8} 85339331$ $9,-0.3108390354 \backslash C, 0.7239711666,0.6365971656,-0.1942003947 \backslash C,-1.7004604$ $583,0.4182589416,0.0737068581 \backslash C,-0.4806032598,1.2042933016,0.588583019$ $8 \backslash \mathrm{H},-0.3541603677,1.0534286902,1.6652093936 \backslash \mathrm{H},-0.6024318735,2.27560972$ $26,0.4211459548 \backslash \mathrm{C},-2.3081098652,1.0230565891,-1.2180605205 \backslash \mathrm{C}, 1.0369168$ $581,-1.5695830757,-1.5508850351 \backslash \mathrm{C},-2.7865328232,0.1089811772,1.1101602$ $926 \backslash \mathrm{C}, 0.7852068301,-1.6901149219,0.9819146471 \backslash \mathrm{H}, 2.1258785356,-1.509930$ 
$4717,-1.4760854214 \backslash \mathrm{H}, 0.7430791019,-2.6207686365,-1.6000473082 \backslash \mathrm{H}, 0.7218$ $229755,-1.0649917725,-2.4697579343 \backslash 0,-1.6845400181,-1.8328492634,-0.72$ $13294781 \backslash \mathrm{H}, 1.8724251765,-1.7126990884,1.0755340472 \backslash \mathrm{H}, 0.3666959655,-1.2$ $31739969,1.8823651405 \backslash \mathrm{H}, 0.4040935764,-2.7099858326,0.8866470379 \backslash \mathrm{H},-3.0$ $555265612,0.3491911682,-1.645012532 \backslash \mathrm{H},-2.7973429663,1.9604279168,-0.93$ $82530417 \backslash \mathrm{H},-1.5500223568,1.24576493,-1.9745800218 \backslash \mathrm{H},-3.5759980267,-0.5$ $164104829,0.6857903939 \backslash \mathrm{H},-2.3705118786,-0.3838156732,1.9939318839 \backslash \mathrm{H},-3$ $.2305695217,1.0575019391,1.4264190203 \backslash \mathrm{C}, 2.1006278102,0.9822135945,0.36$ $43195839 \backslash 0,3.0551786336,0.2407469434,0.3384161308 \backslash \mathrm{H}, 0.7155109833,1.058$ $8662186,-1.2092486679 \backslash 0,2.1193129316,2.2368488771,0.8387866434 \backslash \mathrm{H}, 3.030$ $2432213,2.4337736369,1.1362637793 \backslash \backslash$ Version=IA32L-G03RevC.02 $\backslash$ State=1-A $\backslash$ $\mathrm{HF}=-632.7216205 \backslash \mathrm{S} 2=0 . \backslash \mathrm{S} 2-1=0 . \backslash \mathrm{S} 2 \mathrm{~A}=0 . \backslash \mathrm{RMSD}=9.244 \mathrm{e}-09 \backslash \mathrm{RMSF}=7.876 \mathrm{e}-06 \backslash \mathrm{Dip}$ $\mathrm{ole}=-1.1574248,0.3335607,-0.0004303 \backslash \mathrm{PG}=\mathrm{C} 01 \quad[\mathrm{X}(\mathrm{C} 9 \mathrm{H} 16 \mathrm{N1O3})] \backslash \backslash @$

\section{3-carboxy-PROXYL-}

$1 \backslash 1 \backslash G I N C-L C 28 \backslash F O p t \backslash U B 3 L Y P \backslash 6-31 G(d) \backslash C 9 H 16 N 103(1-) \backslash J O H 501 \backslash 27-F e b-2006 \backslash 0 \backslash$

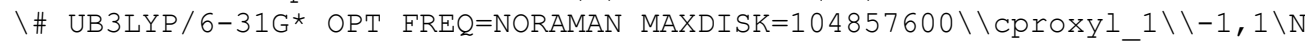
$, 1.1876159806,0.744162652,-0.4111995335 \backslash \mathrm{C},-0.2383292405,0.91 \overline{8} 7218921,0$ $.0133212051 \backslash \mathrm{C},-0.8495230052,-0.391059113,-0.5724863323 \backslash \mathrm{C}, 1.5727721475$, $-0.6948769162,-0.1712605534 \backslash C, 0.2325718768,-1.4630776747,-0.3093519427$ $\backslash \mathrm{H},-0.0102203577,-2.0031480243,0.6106516287 \backslash \mathrm{H}, 0.2479000146,-2.20702034$ $15,-1.1176909368 \backslash \mathrm{C}, 2.5781671321,-1.0356531894,-1.2851327645 \backslash \mathrm{C},-0.73843$ $52062,2.1906429134,-0.6776128823 \backslash C, 2.262354391,-0.8946770876,1.1944104$ $036 \backslash \mathrm{C},-0.3860560325,1.0712585128,1.5432092002 \backslash \mathrm{H},-1.7751293038,2.436908$ $7564,-0.416040081 \backslash \mathrm{H},-0.0657434084,2.9976962383,-0.3680291597 \backslash \mathrm{H},-0.6568$ $782064,2.085931211,-1.7664912031 \backslash 0,2.0294903179,1.66235082,0.184086784$ $\backslash \mathrm{H},-1.2807790441,1.6380302204,1.8353782242 \backslash \mathrm{H},-0.4151901961,0.108702585$ $2,2.064292453 \backslash \mathrm{H}, 0.5192509965,1.6230998975,1.835846245 \backslash \mathrm{H}, 3.4006650917,-$ $0.313529178,-1.2254488557 \backslash \mathrm{H}, 2.9832420633,-2.0549502952,-1.1939376999 \backslash \mathrm{H}$ $, 2.1060011795,-0.925115511,-2.2689025818 \backslash \mathrm{H}, 2.8139324443,0.0400201891,1$ $.366780473 \backslash \mathrm{H}, 1.5424870392,-1.0284671082,2.0097180804 \backslash \mathrm{H}, 2.9424323153,-1$ $.757884354,1.1984758357 \backslash \mathrm{C},-2.1962373469,-0.7367853841,-0.0079927399 \backslash 0$, $-2.4634560533,-1.5573766637,0.8540945352 \backslash \mathrm{H},-0.9701312318,-0.2221658478$ $,-1.6483579546 \backslash 0,-3.2053969495,0.0026772193,-0.5768185545 \backslash \mathrm{H},-4.0039580$ $783,-0.2754217261,-0.0913714133 \backslash \backslash$ Version=IA32L-G03RevC.02 $\backslash$ State=1-A $\backslash H F$ $=-632.967454 \backslash \mathrm{S} 2=0 . \backslash \mathrm{S} 2-1=0 . \backslash \mathrm{S} 2 \mathrm{~A}=0 . \backslash \mathrm{RMSD}=3.243 \mathrm{e}-09 \backslash \mathrm{RMSF}=1.981 \mathrm{e}-05 \backslash \mathrm{Dipole}$ $=-2.0839849,-1.2479011,-0.3968274 \backslash \mathrm{PG}=\mathrm{C} 01 \quad[\mathrm{X}(\mathrm{C} 9 \mathrm{H} 16 \mathrm{~N} 103)] \backslash \backslash @$

\section{3-amino-PROXYL・(16)}

$1 \backslash 1 \backslash G I N C-L C 108 \backslash F O p t \backslash U B 3 L Y P \backslash 6-31 G(d) \backslash C 8 H 17 N 201(2) \backslash J O H 501 \backslash 27-F e b-2006 \backslash 0 \backslash$ \\# UB3LYP/6-31G* OPT FREQ=NORAMAN MAXDISK=104857600 \nproxyl_2 $\backslash \backslash 0,2 \backslash N$, $-0.3495353695,-0.8589101749,-0.0700828306 \backslash C, 1.1389013038,-0 . \overline{8} 179943903$ $,-0.158823541 \backslash C, 1.402910719,0.7003991655,-0.168022312 \backslash C,-1.0142699442$, $0.4535670019,0.1310149654 \backslash \mathrm{C}, 0.2014522042,1.337968071,0.5554325684 \backslash \mathrm{H}, 0$. $3357463554,1.2018465311,1.6366004123 \backslash \mathrm{N}, 0.1386399402,2.7755360462,0.325$ $89098 \backslash \mathrm{C},-1.6585898649,0.8939211439,-1.1962771225 \backslash \mathrm{C}, 1.5944600428,-1.532$ $6124106,-1.4369497989 \backslash C,-2.0804237842,0.3291083517,1.2230058956 \backslash C, 1.72$ $61743439,-1.5171780602,1.0813331799 \backslash \mathrm{H}, 2.6875612766,-1.5233791344,-1.51$ $02979345 \backslash \mathrm{H}, 1.2495924369,-2.5704019131,-1.4283456647 \backslash \mathrm{H}, 1.1838764862,-1$. $0437903865,-2.327091886 \backslash 0,-1.007214035,-1.9435681922,-0.2131072667 \backslash \mathrm{H}, 2$ $.8180953359,-1.5749643044,1.0093281763 \backslash \mathrm{H}, 1.4711281166,-0.9812775598,2$. $0022142234 \backslash \mathrm{H}, 1.3245786125,-2.5325578731,1.1545543107 \backslash \mathrm{H},-2.3458675632,0$ $.1126689087,-1.534942101 \backslash \mathrm{H},-2.224939166,1.8238932889,-1.0730917448 \backslash \mathrm{H},-$ $0.9114446188,1.0469488521,-1.9837337012 \backslash \mathrm{H},-2.8424780775,-0.3979790436$, $0.9287394667 \backslash \mathrm{H},-1.6361818517,-0.0074762617,2.165983868 \backslash \mathrm{H},-2.5673293932$ $, 1.2965641627,1.3946794636 \backslash \mathrm{H}, 1.4452711927,1.0697867438,-1.2012309093 \backslash \mathrm{H}$ , $2.3481862616,0.9692455793,0.3116458177 \backslash \mathrm{H},-0.0301130769,2.9721234259,-$ $0.6593437621 \backslash \mathrm{H},-0.6353921634,3.1878401857,0.8442500436 \backslash \backslash$ Version=IA32LG03RevC.02 State $=2-A \backslash H F=-499.7528128 \backslash S 2=0.753773 \backslash S 2-1=0 . \backslash S 2 A=0.75001 \backslash R$ $\mathrm{MSD}=7.698 \mathrm{e}-09 \backslash \mathrm{RMSF}=1.309 \mathrm{e}-05 \backslash \mathrm{Dipole}=0.1652956,0.867629,-0.0375614 \backslash \mathrm{PG}=\mathrm{C}$ $01[\mathrm{X}(\mathrm{C} 8 \mathrm{H} 17 \mathrm{~N} 2 \mathrm{O} 1)] \backslash \backslash \mathrm{Q}$ 


\section{3-amino-PROXYL+}

$1 \backslash 1 \backslash G I N C-L C 139 \backslash$ FOpt \UB3LYP \6-31G (d) \C8H17N2O1 (1+) \JOH501\08-Mar-2006\0

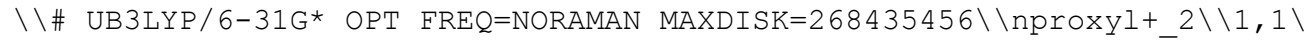
$\mathrm{N}, 0.4632290834,0.8273534504,-0.0528720568 \backslash \mathrm{C}, 1.3667120724,-0.4006677419$ $, 0.0604462232 \backslash C, 0.3328383582,-1.5244243672,0.2430636055 \backslash C,-0.998856341$ $, 0.5172315451,-0.0520825625 \backslash C,-0.9819087339,-1.0116129468,-0.400037671$ $8 \backslash \mathrm{H},-0.9001304268,-1.0844031134,-1.4907259667 \backslash \mathrm{N},-2.2181681405,-1.64760$ $48978,-0.0190821865 \backslash \mathrm{C},-1.4744812407,0.7727492839,1.4035998996 \backslash \mathrm{C}, 2.3374$ $810449,-0.1861810043,1.2299084597 \backslash C,-1.7409419777,1.4089225701,-1.0434$ $756796 \backslash \mathrm{C}, 2.1201814056,-0.4759199586,-1.2891373909 \backslash \mathrm{H}, 2.9801863362,-1.06$ $8330437,1.302760823 \backslash \mathrm{H}, 2.9702442068,0.6893728152,1.0626479889 \backslash \mathrm{H}, 1.80636$ $90556,-0.0715899688,2.179474529 \backslash 0,0.9021555817,1.9318553899,-0.1689774$ $39 \backslash \mathrm{H}, 2.7889430523,-1.3402183303,-1.2423733089 \backslash \mathrm{H}, 1.4428563406,-0.614439$ $2048,-2.1370491374 \backslash \mathrm{H}, 2.7197211423,0.4234743173,-1.4511436151 \backslash \mathrm{H},-1.3979$ $1513,1.8362507141,1.6436522248 \backslash \mathrm{H},-2.5231877544,0.4635752891,1.43607584$ $56 \backslash \mathrm{H},-0.9171978875,0.1982452494,2.1489152971 \backslash \mathrm{H},-1.7150438033,2.4598864$ $658,-0.7460165147 \backslash \mathrm{H},-1.3281493314,1.3134290185,-2.0528994534 \backslash \mathrm{H},-2.7818$ $253289,1.074100881,-1.0631142214 \backslash \mathrm{H}, 0.1755809871,-1.7281744196,1.308316$ $2902 \backslash \mathrm{H}, 0.6750627713,-2.4525613224,-0.2214753264 \backslash \mathrm{H},-2.4896709974,-2.386$ $2552885,-0.6591613878 \backslash \mathrm{H},-2.204662013,-2.026029935,0.9239058489 \backslash \backslash$ Versio $\mathrm{n}=\mathrm{IA} 32 \mathrm{~L}-\mathrm{G} 03 \mathrm{RevC} .02 \backslash \mathrm{State}=1-\mathrm{A} \backslash \mathrm{HF}=-499.5061071 \backslash \mathrm{S} 2=0 . \backslash \mathrm{S} 2-1=0 . \backslash \mathrm{S} 2 \mathrm{~A}=0 . \backslash \mathrm{RMSD}$ $=9.655 e-09 \backslash \mathrm{RMSF}=1.399 \mathrm{e}-05 \backslash \mathrm{Dipole}=0.2379476,-0.7164869,0.0828518 \backslash \mathrm{PG}=\mathrm{C} 01$ $[\mathrm{X}(\mathrm{C} 8 \mathrm{H} 17 \mathrm{~N} 2 \mathrm{O} 1)] \backslash \backslash \mathrm{C}$

\section{3-amino-PROXYL-}

$1 \backslash 1 \backslash G I N C-A C 33 \backslash F O p t \backslash U B 3 L Y P \backslash 6-31 G(d) \backslash C 8 H 17 N 2 O 1(1-) \backslash J O H 501 \backslash 28-F e b-2006 \backslash 0 \backslash$

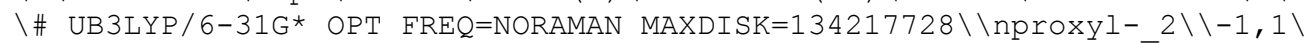
$\mathrm{N}, 0.4422430578,0.7766441264,-0.4740184313 \backslash \mathrm{C}, 1.3087677789,-0.3 \overline{8} 48893733$ $,-0.0427957931 \backslash \mathrm{C}, 0.3272233572,-1.5864918865,-0.0253224229 \backslash \mathrm{C},-0.9543794$ $425,0.4718275305,-0.0679072781 \backslash C,-1.0458288578,-1.0217776472,-0.453782$ $493 \backslash \mathrm{H},-1.1268932088,-1.0723003434,-1.5474124517 \backslash \mathrm{N},-2.1605745339,-1.831$ $5374346,0.0809921342 \backslash \mathrm{C},-1.2035147127,0.7684370627,1.4278056735 \backslash \mathrm{C}, 1.997$ $9700601,-0.1165370142,1.3119996023 \backslash C,-1.8582673142,1.3805023894,-0.910$ $0204513 \backslash \mathrm{C}, 2.3911557779,-0.5072362768,-1.1307028009 \backslash \mathrm{H}, 2.9721600306,-0.6$ $177240882,1.3993460244 \backslash \mathrm{H}, 2.1137083158,0.9782898225,1.3374727362 \backslash \mathrm{H}, 1.38$ $32455944,-0.425411146,2.1663561042 \backslash 0,0.8896144501,2.0166655306,-0.0341$ $022378 \backslash \mathrm{H}, 3.1339752286,-1.2882946619,-0.9054734261 \backslash \mathrm{H}, 1.9301358442,-0.72$ $2043915,-2.1025104164 \backslash \mathrm{H}, 2.897154025,0.4621805814,-1.2070860261 \backslash \mathrm{H},-0.57$ $83711984,1.655429062,1.6148014667 \backslash \mathrm{H},-2.2538928925,0.9896851693,1.66606$ $98702 \backslash \mathrm{H},-0.8722485537,-0.0433360478,2.088754062 \backslash \mathrm{H},-1.5412302857,2.4120$ $501343,-0.7201270067 \backslash \mathrm{H},-1.7097985101,1.1765421895,-1.9780159408 \backslash \mathrm{H},-2.9$ $263603164,1.2685582397,-0.667100061 \backslash \mathrm{H}, 0.2452130051,-2.0027982651,0.988$ $6254368 \backslash \mathrm{H}, 0.6380137256,-2.4166699856,-0.6739396713 \backslash \mathrm{H},-2.1370422309,-1$. $749598663,1.0995166915 \backslash \mathrm{H},-3.0351237223,-1.3766378778,-0.1909196292 \backslash \backslash \mathrm{Ve}$ rsion $=I A 64 \mathrm{~L}-\mathrm{G} 03 \mathrm{RevC} .02 \backslash$ State $=1-\mathrm{A} \backslash \mathrm{HF}=-499.7367436 \backslash \mathrm{S} 2=0 . \backslash \mathrm{S} 2-1=0 . \backslash \mathrm{S} 2 \mathrm{~A}=0 . \backslash$ $\mathrm{RMSD}=5.831 \mathrm{e}-09 \backslash \mathrm{RMSF}=8.345 \mathrm{e}-06 \backslash \mathrm{Dipole}=-1.0587951,-1.585227,0.2780373 \backslash \mathrm{PG}$ $=\mathrm{C} 01 \quad[\mathrm{X}(\mathrm{C} 8 \mathrm{H} 17 \mathrm{~N} 2 \mathrm{O} 1)] \backslash \backslash \mathrm{Q}$

\section{3-methoxy-PROXYL・(17)}

$1 \backslash 1 \backslash G I N C-L C 32 \backslash F O p t \backslash U B 3 L Y P \backslash 6-31 G(d) \backslash C 9 H 18 N 1 O 2(2) \backslash J O H 501 \backslash 27-F e b-2006 \backslash 0 \backslash \backslash$

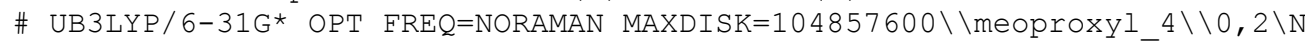
$,-0.6483395965,-0.9504459557,0.1199905662 \backslash \mathrm{C}, 0.8377463004,-0.8812272523$ $, 0.0856085387 \backslash \mathrm{C}, 1.0840291588,0.6467862371,0.1136756076 \backslash \mathrm{C},-1.3420220581$ $, 0.3240091367,0.4547771485 \backslash \mathrm{C},-0.1502420238,1.2218555475,0.8493912846 \backslash \mathrm{H}$ $, 0.0141692931,1.1766611439,1.9322501079 \backslash \mathrm{H},-0.3194734387,2.271464067,0$. $5909227023 \backslash \mathrm{C},-2.0834706014,0.8277432402,-0.7975136933 \backslash \mathrm{C}, 1.3608694607,-$ $1.5961145286,-1.1632017683 \backslash C,-2.3291909663,0.0823942529,1.6027191181 \backslash \mathrm{C}$ $, 1.3926372308,-1.5551783413,1.3570378207 \backslash \mathrm{H}, 2.4554777163,-1.5728536376$, $-1.1857127256 \backslash \mathrm{H}, 1.0272618761,-2.6372497405,-1.1416711314 \backslash \mathrm{H}, 0.986246784$ $9,-1.1234181546,-2.0721705259 \backslash 0,-1.278611543,-2.0487370521,-0.03385415$ $05 \backslash \mathrm{H}, 2.4867223133,-1.6057284008,1.3238904972 \backslash \mathrm{H}, 1.1003397991,-1.0167886$ $843,2.2654891537 \backslash \mathrm{H}, 0.9957917265,-2.5726101769,1.4235961425 \backslash \mathrm{H},-2.783150$ 
$6601,0.0594116756,-1.1417577005 \backslash \mathrm{H},-2.6495921364,1.7383014892,-0.568032$ $2878 \backslash \mathrm{H},-1.3771607088,1.0469438199,-1.6027095752 \backslash \mathrm{H},-3.0639840957,-0.672$ $4669188,1.3093833349 \backslash \mathrm{H},-1.8113718494,-0.2766617316,2.4988850656 \backslash \mathrm{H},-2.8$ $556150928,1.0097980924,1.8542644681 \backslash 0,1.1885237056,1.1374753288,-1.218$ $2932587 \backslash \mathrm{H}, 2.015680041,0.8756388696,0.6527100018 \backslash \mathrm{C}, 1.7709785577,2.42135$ $66501,-1.3158853238 \backslash \mathrm{H}, 1.8425081212,2.6559430127,-2.3809217929 \backslash \mathrm{H}, 1.1664$ $141569,3.198638143,-0.8247340512 \backslash \mathrm{H}, 2.7808056753,2.4384429543,-0.876088$ $7702 \backslash \backslash$ Version=IA32L-G03RevC.02 State $=2-A \backslash H F=-558.9253695 \backslash S 2=0.753778 \backslash S$ $2-1=0 . \backslash \mathrm{S} 2 \mathrm{~A}=0.75001 \backslash \mathrm{RMSD}=4.778 \mathrm{e}-09 \backslash \mathrm{RMSF}=1.904 \mathrm{e}-06 \backslash \mathrm{Dipole}=0.781984,1.328$ $4181,0.4398636 \backslash P G=C 01 \quad[X(C 9 H 18 \mathrm{~N} 102)] \backslash \backslash @$

\section{3-methoxy-PROXYL+}

$1 \backslash 1 \backslash G I N C-L C 23 \backslash F O p t \backslash U B 3 L Y P \backslash 6-31 G(d) \backslash C 9 H 18 N 102(1+) \backslash J O H 501 \backslash 09-M a r-2006 \backslash 0 \backslash$ \\# UB3LYP/6-31G* OPT FREQ=NORAMAN MAXDISK=134217728\\meoproxyl+_1 $\backslash \backslash 1,1$ $\backslash \mathrm{N}, 0.962487062,0.4830137728,-0.3887455024 \backslash \mathrm{C},-0.2716020497,1.199 \overline{9} 048316$ $, 0.1089771384 \backslash C,-1.1135054695,0.0080297815,0.6267506857 \backslash C, 1.1195066449$ $,-0.9353537736,0.1403062826 \backslash C,-0.0657135981,-1.0245861166,1.1181489279$ $\backslash \mathrm{H}, 0.2579790961,-0.7935313754,2.1377894618 \backslash \mathrm{H},-0.4891326737,-2.03121150$ $13,1.1301957391 \backslash \mathrm{C}, 0.9739727402,-1.857738769,-1.0955847859 \backslash \mathrm{C},-0.8768490$ $576,2.0377933646,-1.0167253599 \backslash \mathrm{C}, 2.5136317437,-1.0629536781,0.77196751$ $32 \backslash \mathrm{C}, 0.2301747307,2.0952987926,1.276565644 \backslash \mathrm{H},-1.7794069699,2.521556215$ $5,-0.6330092023 \backslash \mathrm{H},-0.1788586496,2.8141885813,-1.3392529204 \backslash \mathrm{H},-1.160356$ $2337,1.4160031326,-1.8665839961 \backslash 0,1.7524281215,0.987672893,-1.12516037$ $82 \backslash \mathrm{H},-0.6352662422,2.6428573283,1.6599867174 \backslash \mathrm{H}, 0.6678039439,1.52344466$ $57,2.0987474601 \backslash \mathrm{H}, 0.9652641058,2.8157885388,0.9082495943 \backslash \mathrm{H}, 1.759959051$ $6,-1.6503048895,-1.8267456886 \backslash \mathrm{H}, 1.0845639854,-2.8881323844,-0.74492529$ $05 \backslash \mathrm{H},-0.0086710378,-1.7467725617,-1.558032705 \backslash \mathrm{H}, 3.2996178252,-0.890155$ $9756,0.0323035656 \backslash \mathrm{H}, 2.6451408946,-0.3700627698,1.6085559181 \backslash \mathrm{H}, 2.617497$ $8829,-2.0824375312,1.1544417522 \backslash 0,-1.8772651536,-0.4766880947,-0.45164$ $32921 \backslash \mathrm{H},-1.7621529462,0.341272905,1.4477627086 \backslash \mathrm{C},-3.0200352164,-1.2511$ $481022,-0.0768425743 \backslash \mathrm{H},-3.5357716909,-1.5036603233,-1.0038987679 \backslash \mathrm{H},-2$. $7348524217,-2.1761381986,0.4408407213 \backslash \mathrm{H},-3.6895539069,-0.6671566373,0$. $5678419805 \backslash \backslash$ Version=IA32L-G03RevC.02\State $=1-A \backslash H F=-558.6805337 \backslash S 2=0 . \backslash S$ $2-1=0 . \backslash \mathrm{S} 2 \mathrm{~A}=0 . \backslash \mathrm{RMSD}=4.045 \mathrm{e}-09 \backslash \mathrm{RMSF}=1.163 \mathrm{e}-05 \backslash \mathrm{Dipole}=0.1324355,-0.101830$ $9,0.7492088 \backslash \mathrm{PG}=\mathrm{CO} 1 \quad[\mathrm{X}(\mathrm{C} 9 \mathrm{H} 18 \mathrm{~N} 102)] \backslash \backslash @$

\section{3-methoxy-PROXYL-}

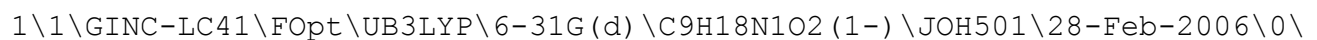

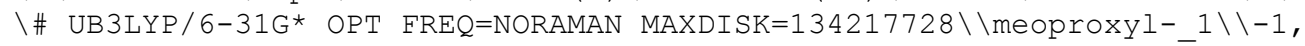
$1 \backslash N,-0.9294124677,0.6851597808,-0.479702944 \backslash \mathrm{C},-1.4878987696,-0 . \overline{6} 112699$ $355,-0.0054775836 \backslash C,-0.296795581,-1.5452592603,-0.2563512132 \backslash \mathrm{C}, 0.51888$ $83119,0.7741230008,-0.0180365102 \backslash C, 0.910066312,-0.718587912,0.20930795$ $94 \backslash \mathrm{H}, 1.0676485297,-0.8824030162,1.2870342302 \backslash 0,2.0881588359,-1.1979440$ $209,-0.4675828227 \backslash C, 1.2511140385,1.4483090401,-1.190690313 \backslash \mathrm{C},-2.706275$ $1045,-0.9068934235,-0.8896796172 \backslash C, 0.6504028303,1.6470769986,1.2460620$ $661 \backslash \mathrm{C},-1.9415492431,-0.5471604578,1.4706329113 \backslash \mathrm{H},-3.2185910257,-1.8381$ $517232,-0.6068201793 \backslash \mathrm{H},-3.3924608925,-0.0589843802,-0.7885443218 \backslash \mathrm{H},-2$. $4019242249,-0.9690301859,-1.9417290776 \backslash 0,-1.7070840498,1.7772646437,-0$ $.1384219902 \backslash \mathrm{H},-2.7175476593,-1.2855473912,1.7187450687 \backslash \mathrm{H},-1.1109676507$ $,-0.6860722399,2.1743968562 \backslash \mathrm{H},-2.3383322085,0.472972671,1.5819386012 \backslash \mathrm{H}$ $, 0.6974364258,2.368324001,-1.4115670142 \backslash \mathrm{H}, 2.2973780322,1.7057882289,-0$ $.9669994042 \backslash \mathrm{H}, 1.2263322393,0.8109075016,-2.0795279178 \backslash \mathrm{H},-0.2262911855$, $2.3150014907,1.1887246338 \backslash \mathrm{H}, 0.5863433987,1.0613042781,2.1726738278 \backslash \mathrm{H}, 1$ $.5836281566,2.2268190539,1.275182558 \backslash \mathrm{H},-0.1958657466,-1.7457949983,-1$. $3295784437 \backslash \mathrm{H},-0.3608218105,-2.5094805023,0.2654523393 \backslash \mathrm{C}, 3.2837821746,-$ $0.8763449922,0.1942900261 \backslash \mathrm{H}, 4.1081928156,-1.2957580452,-0.3956430943 \backslash \mathrm{H}$ , 3.4399840923,0.2087141536,0.292350366\H,3.3227378831,-1.3132506933, 1 . $2095237282 \backslash \backslash$ Version=IA32L-G03RevC.02\State $=1-A \backslash H F=-558.9091651 \backslash S 2=0 . \backslash S$ $2-1=0 . \backslash \mathrm{S} 2 \mathrm{~A}=0 . \backslash \mathrm{RMSD}=7.862 \mathrm{e}-09 \backslash \mathrm{RMSF}=2.878 \mathrm{e}-06 \backslash \mathrm{Dipole}=1.8966887,-1.666180$ $6,0.5103916 \backslash P G=C 01 \quad[X(C 9 H 18 \mathrm{~N} 102)] \backslash \backslash @$ 


\section{3-hydroxy-PROXYL・(18)}

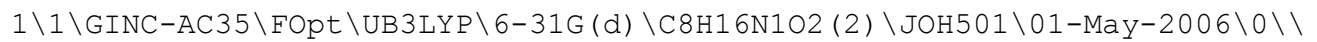
\# UB3LYP/6-31G* OPT FREQ=noraman maxdisk=268435456\\ohproxyl $9 \backslash \backslash 0,2 \backslash N$, $-0.0118882577,-0.0143080881,-0.016699883 \backslash C, 1.4742699315,-0.0 \overline{0} 48632165$, $-0.0351381308 \backslash \mathrm{C}, 1.7808301338,1.5185326032,-0.0282803196 \backslash \mathrm{C},-0.657489075$ $5,1.2936095353,0.2883535476 \backslash \mathrm{C}, 0.5675532503,2.1495944094,0.6765147349 \backslash \mathrm{H}$ $, 0.7188278674,2.1196983365,1.7612197574 \backslash \mathrm{H}, 0.4620571161,3.1964738466,0$. $3797267404 \backslash \mathrm{C},-1.3722643753,1.8018402903,-0.9772511376 \backslash \mathrm{C}, 1.972046383,-0$ $.7528725438,-1.2753606916 \backslash \mathrm{C},-1.6600839917,1.1110065469,1.4341198925 \backslash \mathrm{C}$, $1.9973268972,-0.6864222453,1.244394419 \backslash \mathrm{H}, 3.0682673706,-0.7879123207,-1$ $.2975231878 \backslash \mathrm{H}, 1.5942245561,-1.7782597205,-1.2522113045 \backslash \mathrm{H}, 1.6065426732$, $-0.2814236703,-2.1916994047 \backslash 0,-0.6836365695,-1.0857092288,-0.186338221$ $5 \backslash \mathrm{H}, 3.0895161499,-0.7755024461,1.2208416378 \backslash \mathrm{H}, 1.7195813954,-0.12606015$ $03,2.1438732519 \backslash \mathrm{H}, 1.5652508056,-1.6887095278,1.3214484539 \backslash \mathrm{H},-2.0951362$ $61,1.0517278424,-1.3139639751 \backslash \mathrm{H},-1.9088745213,2.733787873,-0.764479109$ $9 \backslash \mathrm{H},-0.653484259,1.9890403181,-1.7789555574 \backslash \mathrm{H},-2.427505052,0.386681863$ $8,1.1470346 \backslash \mathrm{H},-1.1637189259,0.7417416985,2.3383655603 \backslash \mathrm{H},-2.1454917199$, $2.0643751527,1.6705567971 \backslash 0,1.8566517546,2.0994914462,-1.328522882 \backslash \mathrm{H}, 2$ $.7115486951,1.7159770765,0.5233237029 \backslash \mathrm{H}, 2.6024280291,1.6937543189,-1.7$ $969792902 \backslash \backslash$ Version=IA64L-G03RevD.01 \State $=2-A \backslash H F=-519.6186544 \backslash S 2=0.753$ $784 \backslash \mathrm{S} 2-1=0 . \backslash \mathrm{S} 2 \mathrm{~A}=0.75001 \backslash \mathrm{RMSD}=7.337 \mathrm{e}-09 \backslash \mathrm{RMSF}=5.527 \mathrm{e}-06 \backslash \mathrm{Thermal}=0 . \backslash \mathrm{Dipol}$ $\mathrm{e}=1.0089545,0.5786473,0.3019848 \backslash \mathrm{PG}=\mathrm{C} 01 \quad[\mathrm{X}(\mathrm{C} 8 \mathrm{H} 16 \mathrm{~N} 1 \mathrm{O} 2)] \backslash \backslash @$

\section{3-hydroxy-PROXYL+}

$1 \backslash 1 \backslash G I N C-A C 6 \backslash F O p t \backslash U B 3 L Y P \backslash 6-31 G(d) \backslash C 8 H 16 N 102(1+) \backslash J O H 501 \backslash 01-M a y-2006 \backslash 0 \backslash \backslash$

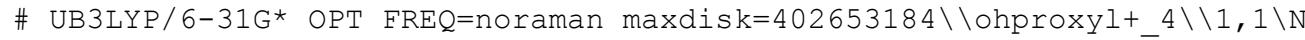
$,-0.189791995,-0.7139504739,-0.421674409 \backslash C, 1.1734366668,-0.2899907817$, $0.0840682266 \backslash C, 0.8549112813,1.1271256171,0.6151610447 \backslash C,-1.3452377628$, $0.1128595658,0.1256319369 \backslash \mathrm{C},-0.6093955044,1.0370666425,1.1133844738 \backslash \mathrm{H}$, $-0.6488550149,0.6264944181,2.1270921074 \backslash \mathrm{H},-1.0695432818,2.0276402875,1$ $.141445636 \backslash \mathrm{C},-1.9414774208,0.8568872153,-1.0955132743 \backslash \mathrm{C}, 2.2021884124,-$ $0.3909407544,-1.0415312624 \backslash \mathrm{C},-2.370506515,-0.8457795099,0.7495176899 \backslash \mathrm{C}$ $, 1.4950541672,-1.2802848855,1.2377102722 \backslash \mathrm{H}, 3.167860797,-0.0604860987,-$ $0.6489470094 \backslash \mathrm{H}, 2.3022818882,-1.4243202204,-1.382243828 \backslash \mathrm{H}, 1.9387640457$, $0.2540667343,-1.8805569185 \backslash 0,-0.3472614394,-1.6237128063,-1.1743088946$ $\backslash \mathrm{H}, 2.4794198703,-1.0107277086,1.6307351395 \backslash \mathrm{H}, 0.7733079144,-1.236449181$ $9,2.057297991 \backslash \mathrm{H}, 1.5405511851,-2.3024495259,0.8528999079 \backslash \mathrm{H},-2.315898305$ $2,0.1443572667,-1.8354859361 \backslash \mathrm{H},-2.781241966,1.4554128847,-0.7300658289$ $\backslash \mathrm{H},-1.2067872478,1.5227962164,-1.551939298 \backslash \mathrm{H},-2.7764560632,-1.53051116$ $15,0.0005039697 \backslash \mathrm{H},-1.93895783,-1.4233606837,1.5724521976 \backslash \mathrm{H},-3.19219981$ $82,-0.2442614281,1.1489075929 \backslash 0,0.993584889,2.0207310181,-0.4732761188$ $\backslash \mathrm{H}, 1.5420691016,1.3765493197,1.431822293 \backslash \mathrm{H}, 1.0371609455,2.9315950342,-$ $0.1404547013 \backslash \backslash$ Version=IA64L-G03RevD.01 \State=1-A \HF=-519.3709095 $\backslash \mathrm{S} 2=0$. $\backslash \mathrm{S} 2-1=0 . \backslash \mathrm{S} 2 \mathrm{~A}=0 . \backslash \mathrm{RMSD}=6.164 \mathrm{e}-09 \backslash \mathrm{RMSF}=2.152 \mathrm{e}-05 \backslash \mathrm{Thermal}=0 . \backslash \mathrm{Dipole}=-0.076$ $1213,0.3367467,0.8730067 \backslash \mathrm{PG}=\mathrm{C} 01 \quad[\mathrm{X}(\mathrm{C} 8 \mathrm{H} 16 \mathrm{~N} 1 \mathrm{O} 2)] \backslash \backslash @$

\section{3-hydroxy-PROXYL-}

$1 \backslash 1 \backslash G I N C-A C 37 \backslash F O p t \backslash U B 3 L Y P \backslash 6-31 G(d) \backslash C 8 H 16 N 102(1-) \backslash J O H 501 \backslash 01-M a y-2006 \backslash 0 \backslash$

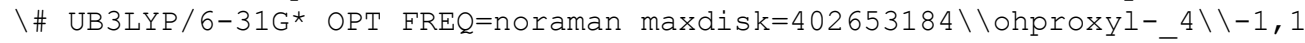
$\backslash \mathrm{N},-0.079768197,-0.2511541814,-0.7620328345 \backslash \mathrm{C}, 1.1450000107,-0 . \overline{1} 9580694$ $, 0.1060674218 \backslash \mathrm{C}, 0.9329219649,1.2242431403,0.6750091837 \backslash \mathrm{C},-1.2447172731$ $, 0.2371643534,0.0920247755 \backslash \mathrm{C},-0.5562450351,1.199372163,1.1000309943 \backslash \mathrm{H}$, $-0.6617226732,0.8277642909,2.1274870792 \backslash \mathrm{H},-0.9610867243,2.2190599025,1$ $.078895838 \backslash \mathrm{C},-2.1933292068,0.9535420526,-0.8843633777 \backslash \mathrm{C}, 2.3559183488,-$ $0.3605414013,-0.8186895362 \backslash C,-2.0061144225,-0.9380856548,0.7367548794 \backslash$ $\mathrm{C}, 1.1910533948,-1.3192823439,1.1622656307 \backslash \mathrm{H}, 3.2975731688,-0.4335625842$ $,-0.2570615309 \backslash \mathrm{H}, 2.1862153975,-1.2795793294,-1.3900807762 \backslash \mathrm{H}, 2.43627292$ $99,0.474191235,-1.5204735193 \backslash 0,-0.2917596972,-1.4773233499,-1.35707579$ $29 \backslash \mathrm{H}, 2.2114961327,-1.52665273,1.513594226 \backslash \mathrm{H}, 0.572579474,-1.1123701075$, $2.042038292 \backslash \mathrm{H}, 0.793042563,-2.2044634746,0.6468030219 \backslash \mathrm{H},-2.4278918686,0$ $.2520242465,-1.6936293237 \backslash \mathrm{H},-3.1282388159,1.2748105448,-0.4009292237 \backslash \mathrm{H}$ $,-1.7086618463,1.833365329,-1.3240436631 \backslash \mathrm{H},-1.8580481177,-1.7703685397$ 
$, 0.0308193473 \backslash \mathrm{H},-1.6017125528,-1.2255289446,1.7140264754 \backslash \mathrm{H},-3.07595041$ $62,-0.7241048201,0.8660541905 \backslash 0,1.1322207439,2.2022938367,-0.355272968$ $7 \backslash \mathrm{H}, 1.6085978838,1.4907059731,1.4982183106 \backslash \mathrm{H}, 0.6993358339,1.7466443336$ ,$-1.1138041194 \backslash \backslash$ Version=IA64L-G03RevD.01 \State $=1-\mathrm{A} \backslash \mathrm{HF}=-519.6119417 \backslash \mathrm{S} 2=$ $0 . \backslash \mathrm{S} 2-1=0 . \backslash \mathrm{S} 2 \mathrm{~A}=0 . \backslash \mathrm{RMSD}=3.429 \mathrm{e}-09 \backslash \mathrm{RMSF}=4.211 \mathrm{e}-06 \backslash \mathrm{Thermal}=0 . \backslash \mathrm{Dipole}=0.02$ $67185,0.8831751,1.3766157 \backslash \mathrm{PG}=\mathrm{C} 01 \quad[\mathrm{X}(\mathrm{C} 8 \mathrm{H} 16 \mathrm{N1O2})] \backslash \backslash \varrho$

\section{2,2,5,5-tetraethylpyrrolidin-1-yloxyl•(19)}

$1 \backslash 1 \backslash G I N C-L C 105 \backslash F O p t \backslash U B 3 L Y P \backslash 6-31 G(d) \backslash C 13 H 24 N 103$ (2) \JOH501\25-Mar-2006\0

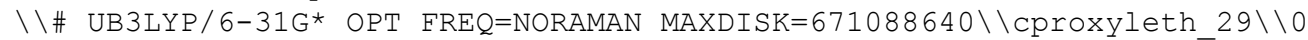
$, 2 \backslash \mathrm{N}, 0.7968701922,0.6111372606,0.0823382428 \backslash \mathrm{C},-0.6719303426,0.85 \overline{6} 96871$ $43,0.1178342852 \backslash C,-1.1842003679,-0.5048293723,-0.4544977914 \backslash C, 1.221707$ $7969,-0.8132888456,-0.0628584756 \backslash C,-0.1388712856,-1.5409772589,-0.0009$ $381109 \backslash \mathrm{H},-0.3667246361,-1.8642237033,1.019008139 \backslash \mathrm{H},-0.160579662,-2.433$ $0310695,-0.633675459 \backslash \mathrm{C}, 1.8887171932,-1.027714489,-1.4498466567 \backslash \mathrm{C},-1.02$ $81214136,2.0900044714,-0.7393279193 \backslash C, 2.1535801762,-1.1681717868,1.115$ $5910312 \backslash \mathrm{C},-1.15005946,1.055203895,1.5876233244 \backslash \mathrm{H},-2.1164019619,2.21592$ $01958,-0.6946651348 \backslash \mathrm{H},-0.5822940727,2.9631788003,-0.2543611212 \backslash \mathrm{C},-0.56$ $51323168,2.0688367508,-2.199237982 \backslash 0,1.635879773,1.5644086318,0.235096$ $2852 \backslash \mathrm{H},-2.2338800789,1.2328273007,1.556708489 \backslash \mathrm{H},-1.0136089843,0.112106$ $3948,2.1298925359 \backslash \mathrm{C},-0.4784449351,2.1836850948,2.3790974063 \backslash \mathrm{C}, 3.217223$ $2788,-0.3026848494,-1.6921570549 \backslash \mathrm{H}, 2.0319983026,-2.1081611255,-1.58120$ $97457 \backslash \mathrm{H}, 1.1673836047,-0.7248793619,-2.2201597238 \backslash \mathrm{H}, 2.9889663792,-0.460$ $6739646,1.1160989069 \backslash \mathrm{H}, 1.5981214101,-0.9846504284,2.0444937279 \backslash \mathrm{C}, 2.675$ $9817392,-2.6090939343,1.1141823373 \backslash \mathrm{C},-2.5911495579,-0.8705175058,-0.03$ $15123151 \backslash 0,-2.8976040711,-1.6968989669,0.7992646096 \backslash \mathrm{H},-1.1895719562,-0$ $.4315469067,-1.5460740195 \backslash 0,-3.5195563447,-0.1438624712,-0.7070876324 \backslash$ $\mathrm{H},-4.3881516542,-0.4287658117,-0.364221763 \backslash \mathrm{H},-0.8560352187,2.181946240$ $7,3.408076362 \backslash \mathrm{H}, 0.6071336735,2.0629070768,2.4084043438 \backslash \mathrm{H},-0.6905389748$ $, 3.1695729127,1.9537159178 \backslash \mathrm{H},-0.8292576886,3.0156764741,-2.6831056702 \backslash$ $\mathrm{H}, 0.5210675275,1.9549110027,-2.2655613933 \backslash \mathrm{H},-1.0364646114,1.2702868171$ $,-2.7834238215 \backslash \mathrm{H}, 3.5587334205,-0.481567291,-2.7182896793 \backslash \mathrm{H}, 3.116317497$ $5,0.7745554822,-1.5406483537 \backslash \mathrm{H}, 4.003158261,-0.6598086669,-1.0175539424$ $\backslash \mathrm{H}, 3.2735889242,-2.7952718958,2.0133371906 \backslash \mathrm{H}, 1.8583073538,-3.339849686$ $3,1.1065665581 \backslash \mathrm{H}, 3.3150839121,-2.8131224646,0.2483813859 \backslash \backslash$ Version=IA32 $\mathrm{L}-\mathrm{G} 03 \mathrm{RevC} .02 \backslash \mathrm{State}=2-\mathrm{A} \backslash \mathrm{HF}=-790.2211675 \backslash \mathrm{S} 2=0.754168 \backslash \mathrm{S} 2-1=0 . \backslash \mathrm{S} 2 \mathrm{~A}=0.75001$ $2 \backslash \mathrm{RMSD}=8.408 \mathrm{e}-09 \backslash \mathrm{RMSF}=2.849 \mathrm{e}-06 \backslash \mathrm{Dipole}=-0.5971661,-0.361735,-0.4130486$ $\backslash \mathrm{PG}=\mathrm{C} 01 \quad[\mathrm{X}(\mathrm{C} 13 \mathrm{H} 24 \mathrm{~N} 103)] \backslash \backslash \mathrm{Q}$

\section{2,2,5,5-tetraethylpyrrolidin-1-yloxyl+}

$1 \backslash 1 \backslash G I N C-L C 81 \backslash F O p t \backslash U B 3 L Y P \backslash 6-31 G(d) \backslash C 13 H 24 N 103(1+) \backslash J O H 501 \backslash 04-A p r-2006 \backslash 0$

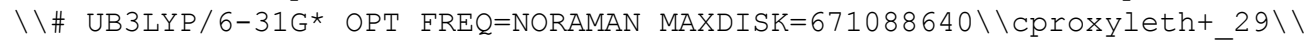
$1,1 \backslash \mathrm{N},-0.5792962195,-0.7270718456,0.3736763603 \backslash \mathrm{C}, 0.9021670349,-0 . \overline{5} 8103$ $52984,0.1951669636 \backslash \mathrm{C}, 0.9609375811,0.6590189288,-0.7432377503 \backslash \mathrm{C},-1.4010$ $296599,0.4463283472,-0.0867930915 \backslash C,-0.3052498952,1.4745013981,-0.4187$ $843329 \backslash \mathrm{H},-0.1127382197,2.1361592508,0.430160423 \backslash \mathrm{H},-0.5964160916,2.1072$ $694286,-1.2597131467 \backslash C,-2.1339572821,-0.0385045968,-1.3931909251 \backslash C, 1.5$ $243583576,-1.8906335872,-0.3347662522 \backslash C,-2.3701545059,0.8497210961,1.0$ $49999358 \backslash \mathrm{C}, 1.4480326102,-0.2260012632,1.6403645932 \backslash \mathrm{H}, 2.5975417637,-1.6$ $859576102,-0.412712794 \backslash \mathrm{H}, 1.4022512946,-2.6650834399,0.426998707 \backslash \mathrm{C}, 0.99$ $51139302,-2.4072029119,-1.6771283525 \backslash 0,-1.066374926,-1.704824659,0.867$ $1238388 \backslash \mathrm{H}, 2.5274786369,-0.1226159404,1.4778773098 \backslash \mathrm{H}, 1.0814091238,0.765$ $2765298,1.9247568275 \backslash C, 1.1905208129,-1.2276264424,2.7638641418 \backslash \mathrm{C},-3.19$ $30566783,-1.1277738378,-1.2436279978 \backslash \mathrm{H},-2.5870129924,0.8783115536,-1.7$ $872758798 \backslash \mathrm{H},-1.3786131791,-0.3412110758,-2.1274979817 \backslash \mathrm{H},-2.9919809607$, $-0.0101924199,1.3149533606 \backslash \mathrm{H},-1.7672361339,1.0947133536,1.9332252534 \backslash \mathrm{C}$ $,-3.2503754247,2.0512799447,0.6874929484 \backslash \mathrm{C}, 2.232854877,1.4872351413,-0$ $.5785641281 \backslash 0,2.2833687485,2.5723220422,-0.0536798597 \backslash \mathrm{H}, 0.9390982016,0$ $.3084868359,-1.7792380197 \backslash 0,3.2962092917,0.8385022049,-1.09522354 \backslash \mathrm{H}, 4$. $0806452347,1.4111067527,-0.978940933 \backslash \mathrm{H}, 1.6522913766,-0.8401854792,3.67$ $84044902 \backslash \mathrm{H}, 0.1257648325,-1.3647468845,2.9821428263 \backslash \mathrm{H}, 1.6297852219,-2.2$ $084953949,2.5664410654 \backslash \mathrm{H}, 1.5157680145,-3.3356556946,-1.9291753439 \backslash \mathrm{H},-0$ $.0746336091,-2.6421010142,-1.6423783791 \backslash \mathrm{H}, 1.1745465019,-1.7085361381$,- 
$2.5003195903 \backslash \mathrm{H},-3.6116123243,-1.3476010976,-2.2314071535 \backslash \mathrm{H},-2.78756379$ $76,-2.0671000754,-0.8520723109 \backslash \mathrm{H},-4.0184660203,-0.8180777832,-0.596813$ $9585 \backslash \mathrm{H},-3.855989372,2.3243088816,1.5567871506 \backslash \mathrm{H},-2.6568891088,2.929436$ $0666,0.4126047502 \backslash \mathrm{H},-3.9389503159,1.828156098,-0.1330757547 \backslash \backslash$ Version=I A32L-G03RevC.02 S State $=1-\mathrm{A} \backslash \mathrm{HF}=-789.9763035 \backslash \mathrm{S} 2=0 . \backslash \mathrm{S} 2-1=0 . \backslash \mathrm{S} 2 \mathrm{~A}=0 . \backslash \mathrm{RMSD}=6$. $206 e-09 \backslash \mathrm{RMSF}=8.432 \mathrm{e}-06 \backslash \mathrm{Dipole}=-0.2571267,-0.4742432,-0.1602499 \backslash \mathrm{PG}=\mathrm{C} 01$ $[\mathrm{X}(\mathrm{C} 13 \mathrm{H} 24 \mathrm{~N} 1 \mathrm{O} 3)] \backslash \backslash \mathrm{Q}$

\section{2,2,5,5-tetraethylpyrrolidin-1-yloxyl-}

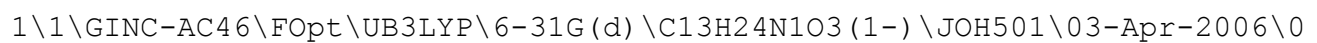

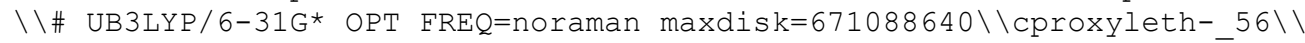
$-1,1 \backslash \mathrm{N}, 0.6965267352,0.9080000404,0.0995971765 \backslash \mathrm{C},-0.7532378883,0.6 \overline{0} 6014$ $7687,0.2098478707 \backslash \mathrm{C},-0.8972030621,-0.3979374552,-0.9827784715 \backslash \mathrm{C}, 1.4609$ $563154,-0.3384723153,-0.2319748308 \backslash \mathrm{C}, 0.3957492762,-1.2407100192,-0.910$ $488408 \backslash \mathrm{H}, 0.2033352385,-2.1404219992,-0.3175569655 \backslash \mathrm{H}, 0.6980216185,-1.58$ $08387312,-1.9104349736 \backslash \mathrm{C}, 2.5529744379,0.0819234107,-1.2540527289 \backslash \mathrm{C},-1$. $5134521058,1.9401787832,0.011986763 \backslash \mathrm{C}, 2.0817168896,-0.9939487116,1.026$ $534263 \backslash \mathrm{C},-1.1184975333,-0.0108378649,1.593445977 \backslash \mathrm{H},-2.5826559931,1.743$ $6355591,-0.1431838852 \backslash \mathrm{H},-1.4107405683,2.4889431962,0.9552751093 \backslash \mathrm{C},-0.9$ $664201035,2.842928196,-1.097323436 \backslash 0,1.1652481658,1.638150142,1.187707$ $2194 \backslash \mathrm{C},-2.558219962,0.1228752756,2.1129431339 \backslash \mathrm{H},-0.8574163678,-1.07578$ $8369,1.6005705742 \backslash \mathrm{H},-0.4189464911,0.5115162788,2.2640336614 \backslash \mathrm{C}, 3.550675$ $3555,1.1141306558,-0.707499289 \backslash \mathrm{H}, 3.0842030003,-0.8038484514,-1.6407613$ $553 \backslash \mathrm{H}, 2.017907547,0.5239317,-2.1049658442 \backslash \mathrm{H}, 2.4935297594,-0.1659370944$ $, 1.6145135968 \backslash \mathrm{H}, 1.2704894832,-1.4090898146,1.6360477692 \backslash \mathrm{C}, 3.116558697$, $-2.0986361672,0.7808046101 \backslash \mathrm{C},-2.1478818965,-1.2281436329,-0.9533276516$ $\backslash 0,-2.2806524431,-2.3713526362,-0.5525800108 \backslash \mathrm{H},-0.9192271385,0.1949650$ $329,-1.9024463696 \backslash 0,-3.2341704235,-0.5513888005,-1.4507453055 \backslash \mathrm{H},-3.977$ $9243086,-1.1706373318,-1.3304558519 \backslash \mathrm{H},-2.6533056471,-0.3632885043,3.09$ $48527099 \backslash \mathrm{H},-2.8556396515,1.1697335301,2.2412360695 \backslash \mathrm{H},-3.2956837735,-0$. $3489671648,1.4501854794 \backslash \mathrm{H},-1.5801463968,3.7522498131,-1.1816037954 \backslash \mathrm{H}, 0$ $.0608435062,3.1195010206,-0.8515496197 \backslash \mathrm{H},-0.9687563318,2.35648083,-2.0$ $812069859 \backslash \mathrm{H}, 4.1153379702,1.5918444915,-1.5215353706 \backslash \mathrm{H}, 2.9985847873,1.8$ $625809494,-0.1276501201 \backslash \mathrm{H}, 4.2813646109,0.6497174524,-0.031424234 \backslash \mathrm{H}, 3.4$ $521510223,-2.5398442865,1.7306343729 \backslash \mathrm{H}, 2.7080108451,-2.9179498636,0.17$ $14630694 \backslash \mathrm{H}, 4.0083648249,-1.7234079128,0.2648520775 \backslash \backslash$ Version=IA64L-G03R evD. $01 \backslash$ State $=1-A \backslash H F=-790.2173444 \backslash \mathrm{S} 2=0 . \backslash \mathrm{S} 2-1=0 . \backslash \mathrm{S} 2 \mathrm{~A}=0 . \backslash \mathrm{RMSD}=4.685 \mathrm{e}-09 \backslash \mathrm{R}$ $\mathrm{MSF}=6.191 \mathrm{e}-06 \backslash \mathrm{Thermal}=0 . \backslash \mathrm{Dipole}=-1.2798937,-1.1592807,-1.0757512 \backslash \mathrm{PG}=\mathrm{C} 0$ $1[\mathrm{X}(\mathrm{C} 13 \mathrm{H} 24 \mathrm{~N} 1 \mathrm{O} 3)] \backslash \backslash @$

\section{3,4-dicarboxy-PROXYL・(20)}

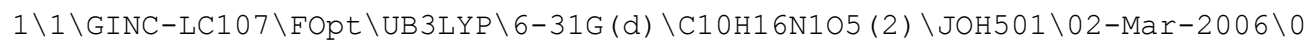

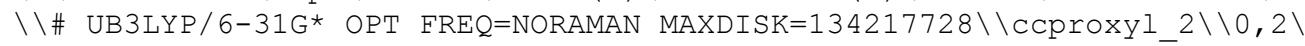
$\mathrm{N},-0.9088773745,-1.4624081311,-0.231795359 \backslash \mathrm{C}, 0.5791462747,-1.453166108$ $7,-0.2637137309 \backslash C, 0.8557624594,0.0798859925,-0.2827131429 \backslash C,-1.5787174$ $27,-0.1551647484,0.0081790645 \backslash C,-0.356554164,0.7232701034,0.4106033826$ $\backslash \mathrm{H},-0.2251757167,0.6692727354,1.4957892957 \backslash \mathrm{C},-0.4976840898,2.188351191$ $6,0.0515887794 \backslash C,-2.2660036744,0.2922538626,-1.2958193119 \backslash C, 1.07140299$ $07,-2.1667676444,-1.5271536747 \backslash C,-2.6073133648,-0.3029269606,1.1342257$ $531 \backslash \mathrm{C}, 1.1025179219,-2.1657862656,0.9977170639 \backslash \mathrm{H}, 2.1639815353,-2.139429$ $5071,-1.5739512936 \backslash \mathrm{H}, 0.7362484617,-3.2074320671,-1.5178489403 \backslash \mathrm{H}, 0.6721$ $016542,-1.6866605213,-2.4267786775 \backslash 0,-1.5755800723,-2.5349240599,-0.40$ $27913305 \backslash \mathrm{H}, 2.1922990237,-2.2680761135,0.9621978886 \backslash \mathrm{H}, 0.8359486667,-1.6$ $247582163,1.9114554645 \backslash \mathrm{H}, 0.6593394753,-3.1646236233,1.0459525585 \backslash \mathrm{H},-2$. $9381346937,-0.5036651524,-1.6292265278 \backslash \mathrm{H},-2.8543747927,1.2014051601,-1$ $.1326854982 \backslash \mathrm{H},-1.5406787714,0.4885031521,-2.0921002939 \backslash \mathrm{H},-3.3707924184$ $,-1.0297262029,0.843939946 \backslash \mathrm{H},-2.1301398248,-0.6567850883,2.0542008267 \backslash$ $\mathrm{H},-3.0842262938,0.6602671475,1.3378859724 \backslash \mathrm{C}, 2.1596531162,0.485796514,0$ $.373351659 \backslash 0,2.2696820995,1.0401732477,1.4448559756 \backslash \mathrm{H}, 0.9004579896,0.4$ $176382789,-1.3228042254 \backslash 0,3.2287601829,0.140730117,-0.3834592174 \backslash \mathrm{H}, 4.0$ $190080349,0.4292015193,0.1120324506 \backslash 0,0.0888072369,2.7570530439,-0.842$ $9252778 \backslash 0,-1.3894955833,2.8161532305,0.8549027231 \backslash \mathrm{H},-1.4243718744,3.74$ $37651646,0.5522505303 \backslash \backslash$ Version=IA32L-G03RevC.02 $\backslash$ State $=2-A \backslash H F=-821.5407$ 
$522 \backslash \mathrm{S} 2=0.753778 \backslash \mathrm{S} 2-1=0 . \backslash \mathrm{S} 2 \mathrm{~A}=0.75001 \backslash \mathrm{RMSD}=6.918 \mathrm{e}-09 \backslash \mathrm{RMSF}=1.015 \mathrm{e}-05 \backslash \mathrm{Dipo}$ $l e=0.2974317,0.4773418,0.0762367 \backslash P G=C 01 \quad[X(C 10 H 16 \mathrm{~N} 105)] \backslash \backslash @$

\section{3,4-dicarboxy-PROXYL+}

$1 \backslash 1 \backslash G I N C-L C 140 \backslash$ FOpt \UB3LYP \6-31G(d) \C10H16N105 (1+) \JOH501\10-Mar-2006 $0 \backslash \backslash \#$ UB3LYP/6-31G* OPT FREQ=NORAMAN MAXDISK=268435456\\ccproxyl+ $1 \backslash \backslash 1$, $1 \backslash \mathrm{N}, 0.00108042,1.7514386696,-0.0002899987 \backslash \mathrm{C}, 1.275797004,0.935036 \overline{4} 25,0$. $1184619623 \backslash \mathrm{C}, 0.6918102094,-0.4845689651,0.3353225694 \backslash \mathrm{C},-1.274615695,0$. $9365513225,-0.118688855 \backslash \mathrm{C},-0.6923592682,-0.4838101094,-0.335327262 \backslash \mathrm{H},-$ $0.5824587604,-0.671698053,-1.4083601077 \backslash C,-1.5770779183,-1.5961928915$, $0.2264555932 \backslash C,-1.9908529525,1.1238171611,1.2462321962 \backslash C, 2.1188005875$, $1.4841485513,1.2742551653 \backslash \mathrm{C},-2.11715735,1.486380659,-1.2744721695 \backslash \mathrm{C}, 1$. $9924557042,1.1211572199,-1.2463842862 \backslash \mathrm{H}, 2.9949876912,0.8386417387,1.37$ $68980524 \backslash \mathrm{H}, 2.4517389756,2.5053861369,1.0739449417 \backslash \mathrm{H}, 1.5660667462,1.460$ $9973054,2.2182979071 \backslash 0,0.0018051709,2.9423113217,-0.0004992341 \backslash \mathrm{H}, 2.941$ $0506235,0.5803664821,-1.189502838 \backslash \mathrm{H}, 1.4166234783,0.7186812668,-2.08419$ $01817 \backslash \mathrm{H}, 2.2032894133,2.1793145633,-1.4202445435 \backslash \mathrm{H},-2.2003616914,2.1822$ $688565,1.4199015343 \backslash \mathrm{H},-2.9401265512,0.5841899666,1.1895850702 \backslash \mathrm{H},-1.415$ $4243899,0.7207747707,2.0840378983 \backslash \mathrm{H},-2.4489655476,2.5080133476,-1.0743$ $041322 \backslash \mathrm{H},-1.5645624575,1.4624691106,-2.2185770342 \backslash \mathrm{H},-2.9940596162,0.84$ $1810614,-1.3769148152 \backslash \mathrm{C}, 1.5750952698,-1.5982686118,-0.2260911196 \backslash 0,1.2$ $909267011,-2.2699939513,-1.1869858884 \backslash \mathrm{H}, 0.5815994852,-0.6721268109,1.4$ $083905429 \backslash 0,2.7098525473,-1.7132057688,0.4864897101 \backslash \mathrm{H}, 3.2314835828,-2$. $449457473,0.1084774562 \backslash 0,-1.294378188,-2.2670766324,1.1883777859 \backslash 0,-2$. $71141797,-1.7106897517,-0.4868302548 \backslash \mathrm{H},-3.2341235581,-2.4459688156,-0$. $1084094739 \backslash \backslash$ Version=IA32L-G03RevC.02\State=1-A \HF=-821.282296\S2=0.\S2 $-1=0 . \backslash \mathrm{S} 2 \mathrm{~A}=0 . \backslash \mathrm{RMSD}=7.953 \mathrm{e}-09 \backslash \mathrm{RMSF}=1.495 \mathrm{e}-05 \backslash \mathrm{Dipole}=0.0012549,1.6072544$, $-0.0004873 \backslash \mathrm{PG}=\mathrm{C} 01 \quad[\mathrm{X}(\mathrm{C} 10 \mathrm{H} 16 \mathrm{~N} 105)] \backslash \backslash \mathrm{Q}$

\section{3,4-dicarboxy-PROXYL-}

$1 \backslash 1 \backslash$ GINC-LC1 05 $\backslash$ FOpt \UB3LYP \6-31G (d) \C10H16N105 (1-) \JOH501 09 -Mar-2006

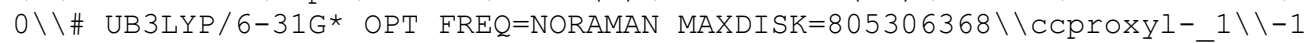
$, 1 \backslash \mathrm{N}, 0.0401925717,1.6478829368,-0.5182127391 \backslash \mathrm{C}, 1.2259522139,0.93 \overline{7} 82668$ $17,0.0884387062 \backslash C, 0.7092456656,-0.5436159826,0.2445234306 \backslash C,-1.2061222$ $313,0.9263009518,-0.1143395941 \backslash C,-0.7313131603,-0.5419680818,-0.301851$ $3324 \backslash \mathrm{H},-0.7150574025,-0.7493896679,-1.3759300822 \backslash \mathrm{C},-1.6046802033,-1.56$ $85038813,0.3603781285 \backslash C,-1.6591522731,1.2806638211,1.3191906449 \backslash \mathrm{C}, 1.66$ $47105733,1.5466887334,1.4359375905 \backslash C,-2.2870353986,1.3514533513,-1.112$ $5792049 \backslash \mathrm{C}, 2.3630662125,1.1290208819,-0.9308402981 \backslash \mathrm{H}, 2.7137380661,1.323$ $0481191,1.6687342034 \backslash \mathrm{H}, 1.5046856719,2.6258855715,1.3214257444 \backslash \mathrm{H}, 1.0486$ $633479,1.1977126347,2.2719115319 \backslash 0,-0.0011008952,2.9920437179,-0.21601$ $36731 \backslash \mathrm{H}, 3.3262416452,0.7360331711,-0.5778674582 \backslash \mathrm{H}, 2.1084262702,0.66089$ $39558,-1.8877096989 \backslash \mathrm{H}, 2.4477155304,2.2083043619,-1.0977118022 \backslash \mathrm{H},-1.381$ $0216909,2.3393381435,1.4247140364 \backslash \mathrm{H},-2.7403935529,1.1608266897,1.46930$ $70278 \backslash \mathrm{H},-1.147939775,0.6897904186,2.0870319195 \backslash \mathrm{H},-2.3504037123,2.44349$ $97446,-1.0572063947 \backslash \mathrm{H},-1.9856471613,1.0834150901,-2.1321479581 \backslash \mathrm{H},-3.26$ $34623466,0.9026251401,-0.8945654985 \backslash \mathrm{C}, 1.570213354,-1.5893347328,-0.412$ $0105034 \backslash 0,1.3830507319,-2.1454360445,-1.4783694927 \backslash \mathrm{H}, 0.6903776408,-0.7$ $904810252,1.3088939592 \backslash 0,2.6806351092,-1.8820113907,0.3311708404 \backslash \mathrm{H}, 3.1$ $729020602,-2.5260960075,-0.2107594182 \backslash 0,-1.3706003137,-2.2006370731,1$. $3770041697 \backslash 0,-2.7815730139,-1.7635803492,-0.3116937005 \backslash \mathrm{H},-3.2627740543$ $,-2.4148082375,0.2314985053 \backslash \backslash$ Version=IA32L-G03RevC.02 $\backslash$ State $=1-A \backslash H F=-82$ $1.5401154 \backslash \mathrm{S} 2=0 . \backslash \mathrm{S} 2-1=0 . \backslash \mathrm{S} 2 \mathrm{~A}=0 . \backslash \mathrm{RMSD}=3.188 \mathrm{e}-09 \backslash \mathrm{RMSF}=4.056 \mathrm{e}-06 \backslash \mathrm{Dipole}=0$. $0088997,-2.568054,0.3182666 \backslash P G=C 01 \quad[X(C 10 H 16 N 105)] \backslash \backslash 0$

\section{3- $\mathrm{NH}_{3}{ }^{+}$-PROXYL $\bullet(21)$}

$1 \backslash 1 \backslash G I N C-A C 30 \backslash F O p t \backslash U B 3 L Y P \backslash 6-31 G(d) \backslash C 8 H 18 N 201(1+, 2) \backslash J O H 501 \backslash 28-A p r-2006 \backslash$

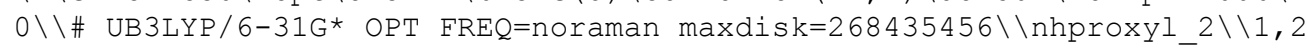
$\backslash \mathrm{N}, 0.3164579013,0.4535938328,0.0906905941 \backslash \mathrm{C}, 1.1176126632,-0.810 \overline{9} 731553$ $, 0.1925030804 \backslash \mathrm{C},-0.004061242,-1.870852846,0.3792678012 \backslash \mathrm{C},-1.1244363891$ $, 0.3122595885,0.4446918143 \backslash C,-1.3366259361,-1.1660926828,0.0588412778 \backslash$ 
$\mathrm{N},-1.6097036885,-1.2781242,-1.4517528653 \backslash \mathrm{C},-1.3513744302,0.5014445066$, $1.9596039345 \backslash \mathrm{C}, 2.0766674815,-0.7370500274,1.3897475409 \backslash \mathrm{C},-1.9468615261$ $, 1.3479837458,-0.3321889279 \backslash \mathrm{C}, 1.9206271101,-0.9839672427,-1.1071617547$ $\backslash \mathrm{H}, 2.6675849874,-1.6560351711,1.459937347 \backslash \mathrm{H}, 2.7587984113,0.1076762704$, $1.2630530684 \backslash \mathrm{H}, 1.5364527376,-0.601464571,2.3316345197 \backslash 0,0.8753539615,1$ $.5963191235,0.0756348641 \backslash \mathrm{H}, 2.5630858459,-1.8681151133,-1.0504783973 \backslash \mathrm{H}$, $1.2778418497,-1.0970926345,-1.9923696851 \backslash H, 2.5488970353,-0.1040653954$, $-1.268956114 \backslash \mathrm{H},-0.9404526202,1.4712115858,2.2529139175 \backslash \mathrm{H},-2.4206567149$ $, 0.4910204399,2.1952159039 \backslash \mathrm{H},-0.8589842502,-0.2704788432,2.5574897445 \backslash$ $\mathrm{H},-1.6590344926,2.3459248759,0.0056541895 \backslash \mathrm{H},-1.7528478723,1.3295319601$ $,-1.4111672364 \backslash \mathrm{H},-3.0195186168,1.2223922781,-0.1472857445 \backslash \mathrm{H},-0.0469392$ $795,-2.2128443299,1.4168392594 \backslash \mathrm{H}, 0.1559078192,-2.7663620239,-0.2324561$ $764 \backslash \mathrm{H},-2.4817481636,-0.8056445607,-1.7172017813 \backslash \mathrm{H},-0.8444778786,-0.837$ $0939757,-1.97948337 \backslash \mathrm{H},-1.6728382716,-2.2591889632,-1.7474518915 \backslash \mathrm{H},-2.2$ $058664318,-1.6213724713,0.5369950873 \backslash \backslash$ Version=IA64L-G03RevD.01 \State=2 $-\mathrm{A} \backslash \mathrm{HF}=-500.116714 \backslash \mathrm{S} 2=0.75351 \backslash \mathrm{S} 2-1=0 . \backslash \mathrm{S} 2 \mathrm{~A}=0.750009 \backslash \mathrm{RMSD}=7.019 \mathrm{e}-09 \backslash \mathrm{RMSF}=$ $4.231 e-06 \backslash$ Thermal $=0 . \backslash$ Dipole $=-2.3840946,-2.3091811,-1.5964995 \backslash P G=C 01 \quad[X$ $(\mathrm{C} 8 \mathrm{H} 18 \mathrm{~N} 2 \mathrm{O} 1)] \backslash \backslash 0$

\section{3-NH ${ }^{+}$-PROXYL+}

$1 \backslash 1 \backslash G I N C-L C 92 \backslash F O p t \backslash U B 3 L Y P \backslash 6-31 G(d) \backslash C 8 H 18 N 2 O 1(2+) \backslash J O H 501 \backslash 14-M a r-2006 \backslash 0 \backslash$

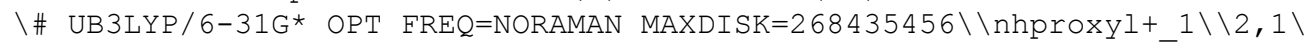
$\mathrm{N}, 0.5219378397,0.823336254,0.0905718733 \backslash \mathrm{C}, 1.398681584,-0.41554 \overline{1} 9801,0$. $0293991223 \backslash \mathrm{C}, 0.3360487415,-1.5286042531,0.1688937718 \backslash \mathrm{C},-0.9836501867,0$ $.5666601491,-0.0617603063 \backslash C,-0.9453657048,-0.9416484311,-0.4516171757 \backslash$ $\mathrm{H},-0.9427185032,-1.0434172173,-1.5399506803 \backslash \mathrm{N},-2.1839049996,-1.7035540$ $835,-0.0016602319 \backslash \mathrm{C},-1.5928314253,0.8653025595,1.3308150297 \backslash \mathrm{C}, 2.446387$ $6337,-0.3628228464,1.1481003127 \backslash C,-1.5401046382,1.5059123834,-1.140673$ $0181 \backslash \mathrm{C}, 2.0637642394,-0.3740401803,-1.3771071198 \backslash \mathrm{H}, 3.0452002341,-1.2773$ $047127,1.0933648492 \backslash \mathrm{H}, 3.1173724588,0.4897539827,1.0181899265 \backslash \mathrm{H}, 1.98306$ $87611,-0.3119588597,2.1380498574 \backslash 0,0.9486199639,1.9198807783,0.2395219$ $454 \backslash \mathrm{H}, 2.6945048149,-1.2650210151,-1.4579466838 \backslash \mathrm{H}, 1.3419095474,-0.38779$ $43734,-2.1994668221 \backslash \mathrm{H}, 2.6972982965,0.5115941652,-1.4704514924 \backslash \mathrm{H},-1.377$ $0439514,1.9012394437,1.6073065638 \backslash \mathrm{H},-2.6833488763,0.7765880531,1.27596$ $10981 \backslash \mathrm{H},-1.2104510145,0.2163408463,2.1264629734 \backslash \mathrm{H},-1.4059881377,2.5505$ $024066,-0.8476760869 \backslash \mathrm{H},-1.0635639309,1.3442633621,-2.1122880496 \backslash \mathrm{H},-2.6$ $16268966,1.3321427827,-1.2490035647 \backslash \mathrm{H}, 0.1859067061,-1.7652342782,1.229$ $7458042 \backslash \mathrm{H}, 0.6615270455,-2.4415320484,-0.3370287405 \backslash \mathrm{H},-2.3236450631,-1$. $6737801148,1.018340163 \backslash \mathrm{H},-3.045331735,-1.3472167908,-0.4418752454 \backslash \mathrm{H},-2$ $.1111987395,-2.6979914581,-0.2665946216 \backslash \backslash$ Version=IA32L-G03RevC.02 $\backslash$ Stat $\mathrm{e}=1-\mathrm{A} \backslash \mathrm{HF}=-499.7253585 \backslash \mathrm{S} 2=0 . \backslash \mathrm{S} 2-1=0 . \backslash \mathrm{S} 2 \mathrm{~A}=0 . \backslash \mathrm{RMSD}=8.315 \mathrm{e}-09 \backslash \mathrm{RMSF}=1.288 \mathrm{e}-$ $05 \backslash \mathrm{Dipole}=-2.5464527,-2.3393753,-0.087912 \backslash \mathrm{PG}=\mathrm{C} 01 \quad[\mathrm{X}(\mathrm{C} 8 \mathrm{H} 18 \mathrm{~N} 2 \mathrm{O} 1)] \backslash \backslash \mathrm{Q}$

\section{2,2,5,5-tetramethyl-3-oxazolidin-1-yloxyl• (22)}

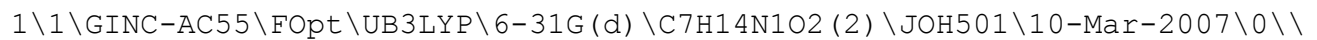

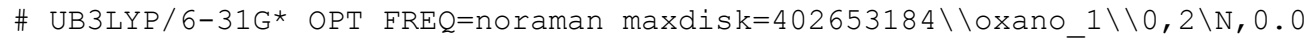
$625615518,-0.0237800102,0.1329503414 \backslash 0,1.3363991188,-0.0103072548,0.12$ $93719984 \backslash \mathrm{C},-0.7621236776,1.2114520936,0.050252412 \backslash \mathrm{C},-0.7540151059,-1.2$ $594747098,0.0276471552 \backslash 0,-2.0663210546,0.6938712128,-0.1972436862 \backslash \mathrm{C},-2$ $.1330632589,-0.6416481684,0.3144212264 \backslash \mathrm{H},-2.9521006821,-1.1476073389,-$ $0.2025996455 \backslash \mathrm{H},-2.3427809184,-0.6374484672,1.3945542337 \backslash \mathrm{C},-0.688788940$ $4,1.9695898603,1.3805041538 \backslash \mathrm{C},-0.6463924209,-1.8260225548,-1.398480509$ $7 \backslash C,-0.3310857425,2.0733004717,-1.1313540736 \backslash C,-0.2918200007,-2.277647$ $3466,1.0705712311 \backslash \mathrm{H},-1.0810720008,1.3581215437,2.1991458541 \backslash \mathrm{H},-1.06826$ $78414,-1.1264559256,-2.1272682316 \backslash \mathrm{H},-1.2736671783,2.8928266114,1.32223$ $5284 \backslash \mathrm{H},-1.1843095004,-2.7776276995,-1.4783178807 \backslash \mathrm{H}, 0.3531792147,2.2179$ $50936,1.6047658854 \backslash \mathrm{H}, 0.405523327,-1.9971690604,-1.6477346027 \backslash \mathrm{H},-0.4502$ $441679,1.5186044725,-2.0659714199 \backslash \mathrm{H},-0.3939596934,-1.8782634007,2.0851$ $482338 \backslash \mathrm{H},-0.9416640628,2.9800789304,-1.1786608182 \backslash \mathrm{H},-0.879562425,-3.19$ $9099033,0.996671485 \backslash \mathrm{H}, 0.7199326086,2.3515233726,-1.0151817175 \backslash \mathrm{H}, 0.7619$ $728512,-2.5202385353,0.9052930913 \backslash \backslash$ Version=IA64L-G03RevD.01 \State=2-A \ $\mathrm{HF}=-480.3070521 \backslash \mathrm{S} 2=0.753716 \backslash \mathrm{S} 2-1=0 . \backslash \mathrm{S} 2 \mathrm{~A}=0.75001 \backslash \mathrm{RMSD}=2.620 \mathrm{e}-09 \backslash \mathrm{RMSF}=4$. $131 \mathrm{e}-05 \backslash$ Thermal $=0 . \backslash \mathrm{Dipole}=-0.7305178,-0.3130759,0.1298462 \backslash \mathrm{PG}=\mathrm{C} 01 \quad[\mathrm{X}(\mathrm{C} 7$ 
$\mathrm{H} 14 \mathrm{~N} 1 \mathrm{O} 2)] \backslash \backslash \mathrm{Q}$

\section{2,2,5,5-tetramethyl-3-oxazolidin-1-yloxyl+}

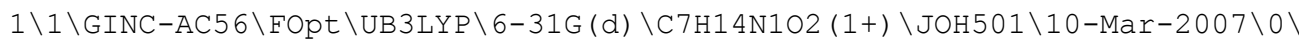

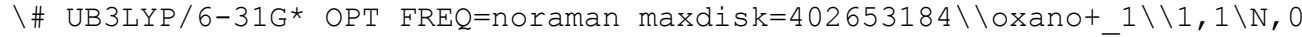
$.092579369,-0.0347513574,0.0666248647 \backslash 0,1.278684826,-0.0253870862,0.13$ $80405048 \backslash \mathrm{C},-0.7861707958,1.2366889086,-0.0104132228 \backslash \mathrm{C},-0.7600234559,-1$ $.2777410266,0.0536242121 \backslash 0,-2.0297982434,0.6733010897,-0.3028171043 \backslash \mathrm{C}$, $-2.133264407,-0.6188126354,0.3119284394 \backslash \mathrm{H},-2.9472652633,-1.1490161336$, $-0.1823166369 \backslash \mathrm{H},-2.3492670323,-0.5389914505,1.3842172094 \backslash \mathrm{C},-0.69678524$ $67,1.8918457716,1.3796288613 \backslash C,-0.6486775126,-1.8329420759,-1.39232456$ $21 \backslash C,-0.3091269534,2.1327242534,-1.1450346755 \backslash C,-0.2636234162,-2.26941$ $96486,1.1042713652 \backslash \mathrm{H},-1.0138135158,1.2201007808,2.1816256437 \backslash \mathrm{H},-1.0422$ $63558,-1.1273583548,-2.1281702466 \backslash \mathrm{H},-1.373222498,2.7514348879,1.365582$ $6078 \backslash \mathrm{H},-1.246886008,-2.7482546626,-1.432152475 \backslash \mathrm{H}, 0.3203197367,2.238364$ $3898,1.579861763 \backslash \mathrm{H}, 0.3893179821,-2.0801026231,-1.6306828298 \backslash \mathrm{H},-0.37880$ $43393,1.6159051763,-2.1051142682 \backslash \mathrm{H},-0.2836576951,-1.8380797871,2.10964$ $09585 \backslash \mathrm{H},-0.9627770375,3.0088051566,-1.1752000609 \backslash \mathrm{H},-0.9251493536,-3.14$ $08687247,1.0915831429 \backslash \mathrm{H}, 0.7171317625,2.4654119063,-0.9709857463 \backslash \mathrm{H}, 0.75$ $08726558,-2.6083267545,0.8793022557 \backslash \backslash$ Version=IA64L-G03RevD.01 \State=1$\mathrm{A} \backslash \mathrm{HF}=-480.0467524 \backslash \mathrm{S} 2=0 . \backslash \mathrm{S} 2-1=0 . \backslash \mathrm{S} 2 \mathrm{~A}=0 . \backslash \mathrm{RMSD}=7.671 \mathrm{e}-09 \backslash \mathrm{RMSF}=3.557 \mathrm{e}-06 \backslash \mathrm{T}$ hermal $=0 . \backslash$ Dipole $=0.0356274,-0.3805908,0.2358717 \backslash \mathrm{PG}=\mathrm{C} 01 \quad[\mathrm{X}(\mathrm{C} 7 \mathrm{H} 14 \mathrm{~N} 1 \mathrm{O} 2)] \backslash$ $\backslash$ @

\section{2,2,5,5-tetramethyl-3-oxazolidin-1-yloxyl-}

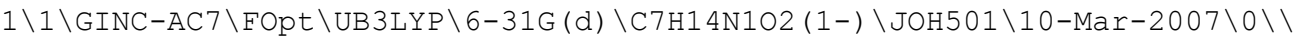
\# UB3LYP/6-31G* OPT FREQ=noraman maxdisk=402653184 \\oxano- $1 \backslash \backslash-1,1 \backslash \mathrm{N}$, $0.007360846,-0.0426982733,0.5840682751 \backslash 0,1.3122977606,-0.0 \overline{3} 7503662,0.1$ $433584682 \backslash C,-0.7956711097,1.1567382866,0.1770833795 \backslash C,-0.7975392662,-1$ $.2163798623,0.1077548858 \backslash 0,-2.1691717998,0.702335695,0.0963057293 \backslash \mathrm{C},-2$ $.2040531149,-0.6952092716,0.3984619989 \backslash \mathrm{H},-2.9851155672,-1.1565167524,-$ $0.2232325616 \backslash \mathrm{H},-2.4685927041,-0.8558111287,1.4583994603 \backslash \mathrm{C},-0.665776212$ $1,2.1861129375,1.3049307456 \backslash \mathrm{C},-0.5427234757,-1.5334629792,-1.380315941$ $8 \backslash \mathrm{C},-0.3401431261,1.7579852961,-1.1613841054 \backslash \mathrm{C},-0.3703165931,-2.405808$ $7676,0.9723589661 \backslash \mathrm{H},-1.0240582339,1.7484726438,2.2425096444 \backslash \mathrm{H},-1.16431$ $67362,-0.9200274369,-2.0424694666 \backslash \mathrm{H},-1.222750034,3.1098361552,1.092920$ $6842 \backslash \mathrm{H},-0.7155634152,-2.5898883942,-1.6306132098 \backslash \mathrm{H}, 0.3989033105,2.4206$ $259427,1.4216701152 \backslash \mathrm{H}, 0.5156947556,-1.2732397019,-1.5308487203 \backslash \mathrm{H},-0.87$ $63279649,1.3008011729,-1.9983713358 \backslash \mathrm{H},-0.5801276236,-2.2035066263,2.02$ $97004027 \backslash \mathrm{H},-0.4844123396,2.8455345352,-1.2108832304 \backslash \mathrm{H},-0.8576147497,-3$ $.3472297229,0.6787475685 \backslash \mathrm{H}, 0.7272748754,1.4955490358,-1.2181458849 \backslash \mathrm{H}, 0$ $.71579421,-2.5021791217,0.8587141327 \backslash \backslash$ Version=IA64L-G03RevD.01 \State $=1$ $-\mathrm{A} \backslash \mathrm{HF}=-480.2978821 \backslash \mathrm{S} 2=0 . \backslash \mathrm{S} 2-1=0 . \backslash \mathrm{S} 2 \mathrm{~A}=0 . \backslash \mathrm{RMSD}=2.467 \mathrm{e}-09 \backslash \mathrm{RMSF}=1.875 \mathrm{e}-06 \backslash$ Thermal $=0 . \backslash$ Dipole $=-1.5763278,-0.2596968,-0.0622258 \backslash P G=C 01 \quad[X(C 7 H 14 N 102$ )$] \backslash \backslash 0$

\section{TMIO•(23)}

$1 \backslash 1 \backslash G I N C-A C 35 \backslash F O p t \backslash U B 3 L Y P \backslash 6-31 G(d) \backslash C 12 H 16 N 1 O 1(2) \backslash J O H 501 \backslash 28-F e b-2006 \backslash 0 \backslash$

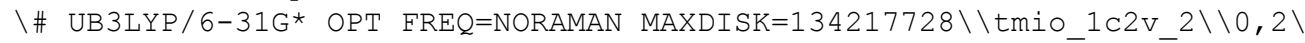
$0,0 ., 0 .,-2.7800581119 \backslash \mathrm{N}, 0 ., 0 .,-1.5057552508 \backslash \mathrm{C}, 1.262504022 \overline{8}, 0 .,-0.71307$ $32792 \backslash C,-1.2625040228,0 .,-0.7130732792 \backslash C, 0.7000380857,0 ., 0.6928829269 \backslash$ $\mathrm{C},-0.7000380857,0 ., 0.6928829269 \backslash \mathrm{C}, 1.4056709073,0 ., 1.8948649635 \backslash \mathrm{C},-1.40$ $56709073,0 ., 1.8948649635 \backslash \mathrm{H}, 2.492899722,0 ., 1.8992572854 \backslash \mathrm{H},-2.492899722$, $0 ., 1.8992572854 \backslash \mathrm{C}, 0.6990894526,0 ., 3.1001372598 \backslash \mathrm{C},-0.6990894526,0 ., 3.10$ $01372598 \backslash \mathrm{H}, 1.2399009592,0 ., 4.0426547966 \backslash \mathrm{H},-1.2399009592,0 ., 4.042654796$ $6 \backslash C,-2.0733777389,-1.2649410868,-1.048711587 \backslash C,-2.0733777389,1.2649410$ $868,-1.048711587 \backslash \mathrm{C}, 2.0733777389,1.2649410868,-1.048711587 \backslash \mathrm{C}, 2.07337773$ $89,-1.2649410868,-1.048711587 \backslash \mathrm{H},-2.2816000136,-1.2877418305,-2.1227719$ $232 \backslash \mathrm{H},-2.2816000136,1.2877418305,-2.1227719232 \backslash \mathrm{H}, 2.2816000136,1.287741$ $8305,-2.1227719232 \backslash \mathrm{H}, 2.2816000136,-1.2877418305,-2.1227719232 \backslash \mathrm{H},-3.023$ $8476994,-1.2687194109,-0.5041274401 \backslash \mathrm{H},-3.0238476994,1.2687194109,-0.50$ 
$41274401 \backslash \mathrm{H}, 3.0238476994,1.2687194109,-0.5041274401 \backslash \mathrm{H}, 3.0238476994,-1.2$ $687194109,-0.5041274401 \backslash \mathrm{H},-1.517448926,-2.168884498,-0.7810348559 \backslash \mathrm{H},-1$ $.517448926,2.168884498,-0.7810348559 \backslash \mathrm{H}, 1.517448926,2.168884498,-0.7810$ $348559 \backslash \mathrm{H}, 1.517448926,-2.168884498,-0.7810348559 \backslash \backslash$ Version=IA64L-G03RevC $.02 \backslash \mathrm{State}=2-\mathrm{B} 1 \backslash \mathrm{HF}=-596.8384326 \backslash \mathrm{S} 2=0.753786 \backslash \mathrm{S} 2-1=0 . \backslash \mathrm{S} 2 \mathrm{~A}=0.750011 \backslash \mathrm{RMSD}=7$ $.719 e-09 \backslash \mathrm{RMSF}=2.533 e-05 \backslash \mathrm{Dipole}=0 ., 0 ., 1.1308772 \backslash \mathrm{PG}=\mathrm{C} 02 \mathrm{~V} \quad[\mathrm{C} 2(\mathrm{~N} 101), \mathrm{SGV}(\mathrm{C}$ $8 \mathrm{H} 4), \mathrm{X}(\mathrm{C} 4 \mathrm{H} 12)] \backslash \backslash \mathrm{C}$

\section{TMIO+}

$1 \backslash 1 \backslash G I N C-L C 26 \backslash$ FOpt \UB3LYP \6-31G (d) \C12H16N1O1 (1+) \JOH501\09-Mar-2006\0

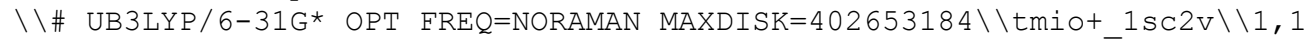
$\backslash 0,0 ., 0 .,-2.7092800943 \backslash \mathrm{N}, 0 ., 0 .,-1.518641855 \backslash \mathrm{C}, 1.2798468357, \overline{0} .,-0.69472$ $16034 \backslash C,-1.2798468357,0 .,-0.6947216034 \backslash C, 0.6992916558,0 ., 0.6990069409 \backslash$ $\mathrm{C},-0.6992916558,0 ., 0.6990069409 \backslash \mathrm{C}, 1.4104694561,0 ., 1.897156315 \backslash \mathrm{C},-1.410$ $4694561,0 ., 1.897156315 \backslash \mathrm{H}, 2.4967794342,0 ., 1.9055739227 \backslash \mathrm{H},-2.4967794342$, $0 ., 1.9055739227 \backslash \mathrm{C}, 0.6993477692,0 ., 3.0988591682 \backslash \mathrm{C},-0.6993477692,0 ., 3.09$ $88591682 \backslash \mathrm{H}, 1.2390046367,0 ., 4.0405425606 \backslash \mathrm{H},-1.2390046367,0 ., 4.040542560$ $6 \backslash \mathrm{C},-2.0639223492,-1.2768540451,-1.0739763506 \backslash \mathrm{C},-2.0639223492,1.276854$ $0451,-1.0739763506 \backslash \mathrm{C}, 2.0639223492,1.2768540451,-1.0739763506 \backslash \mathrm{C}, 2.06392$ $23492,-1.2768540451,-1.0739763506 \backslash \mathrm{H},-2.338931127,-1.2641439429,-2.1321$ $359468 \backslash \mathrm{H},-2.338931127,1.2641439429,-2.1321359468 \backslash \mathrm{H}, 2.338931127,1.26414$ $39429,-2.1321359468 \backslash \mathrm{H}, 2.338931127,-1.2641439429,-2.1321359468 \backslash \mathrm{H},-2.977$ $6275281,-1.2927083897,-0.4733578647 \backslash \mathrm{H},-2.9776275281,1.2927083897,-0.47$ $33578647 \backslash \mathrm{H}, 2.9776275281,1.2927083897,-0.4733578647 \backslash \mathrm{H}, 2.9776275281,-1.2$ $927083897,-0.4733578647 \backslash \mathrm{H},-1.4926417092,-2.1811719974,-0.8484253537 \backslash \mathrm{H}$, $-1.4926417092,2.1811719974,-0.8484253537 \backslash \mathrm{H}, 1.4926417092,2.1811719974,-$ $0.8484253537 \backslash \mathrm{H}, 1.4926417092,-2.1811719974,-0.8484253537 \backslash \backslash$ Version=IA32L $-\mathrm{G} 03 \mathrm{RevC} .02 \backslash \mathrm{State}=1-\mathrm{A} 1 \backslash \mathrm{HF}=-596.5876311 \backslash \mathrm{S} 2=0 . \backslash \mathrm{S} 2-1=0 . \backslash \mathrm{S} 2 \mathrm{~A}=0 . \backslash \mathrm{RMSD}=7.245$ $e-09 \backslash \mathrm{RMSF}=2.489 \mathrm{e}-05 \backslash \mathrm{Dipole}=0 ., 0 .,-0.4990142 \backslash \mathrm{PG}=\mathrm{C} 02 \mathrm{~V} \quad[\mathrm{C} 2(\mathrm{~N} 1 \mathrm{O} 1), \mathrm{SGV}(\mathrm{C} 8 \mathrm{H} 4$ )$, \mathrm{X}(\mathrm{C} 4 \mathrm{H} 12)] \backslash \backslash @$

\section{TMIO-}

$1 \backslash 1 \backslash$ GINC-LC50 \FOpt \UB3LYP \6-31G (d) \C12H16N1O1 (1-) \JOH501\01-Mar-2006\0

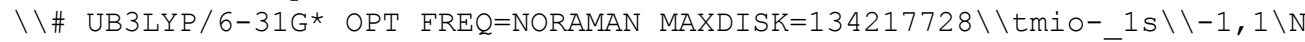
$,-1.2959709722,0.815365982,0 . \backslash 0,-2.6721222399,0.8153731662, \overline{0} . \backslash \mathrm{C}, 3.0427$ $016672,-0.7425144269,0.6981314018 \backslash \mathrm{C}, 3.0427016672,-0.7425144269,-0.6981$ $314018 \backslash \mathrm{C}, 1.8724394054,-0.4272652771,1.4046137864 \backslash \mathrm{C}, 1.8724394054,-0.427$ $2652771,-1.4046137864 \backslash \mathrm{C}, 0.7095568392,-0.1137160947,0.7023079663 \backslash \mathrm{C}, 0.70$ $95568392,-0.1137160947,-0.7023079663 \backslash C,-0.6761697967,0.1967445458,1.21$ $88550328 \backslash C,-0.6761697967,0.1967445458,-1.2188550328 \backslash C,-1.4478464799,-1$ $.059760925,1.6962171938 \backslash \mathrm{C},-1.4478464799,-1.059760925,-1.6962171938 \backslash \mathrm{C},-$ $0.7265493403,1.2686169314,2.3208293555 \backslash C,-0.7265493403,1.2686169314,-2$ $.3208293555 \backslash \mathrm{H}, 3.9537794805,-0.9920916113,1.2405046741 \backslash \mathrm{H}, 3.9537794805,-$ $0.9920916113,-1.2405046741 \backslash \mathrm{H}, 1.8740475674,-0.4326839102,2.4933701152 \backslash \mathrm{H}$ $, 1.8740475674,-0.4326839102,-2.4933701152 \backslash \mathrm{H},-1.1421940808,-1.962618697$ $7,1.1558504559 \backslash \mathrm{H},-1.1421940808,-1.9626186977,-1.1558504559 \backslash \mathrm{H},-1.334238$ $7695,-1.25047431,2.7719189061 \backslash \mathrm{H},-1.3342387695,-1.25047431,-2.771918906$ $1 \backslash \mathrm{H},-2.495878317,-0.8316286182,1.4506194775 \backslash \mathrm{H},-2.495878317,-0.83162861$ $82,-1.4506194775 \backslash \mathrm{H},-0.3440613909,0.9066091102,3.2863251608 \backslash \mathrm{H},-0.344061$ $3909,0.9066091102,-3.2863251608 \backslash \mathrm{H},-0.1557947961,2.1520739016,2.0141856$ $166 \backslash \mathrm{H},-0.1557947961,2.1520739016,-2.0141856166 \backslash \mathrm{H},-1.7760661017,1.56291$ $20117,2.4332985733 \backslash \mathrm{H},-1.7760661017,1.5629120117,-2.4332985733 \backslash \backslash$ Version $=I A 32 \mathrm{~L}-\mathrm{G} 03 \mathrm{RevC} .02 \backslash$ State $=1-\mathrm{A}^{\prime} \backslash \mathrm{HF}=-596.828569 \backslash \mathrm{S} 2=0 . \backslash \mathrm{S} 2-1=0 . \backslash \mathrm{S} 2 \mathrm{~A}=0 . \backslash \mathrm{RMSD}=$ $7.733 e-09 \backslash \mathrm{RMSF}=3.382 \mathrm{e}-06 \backslash \mathrm{Dipole}=2.4519024,-0.8595516,0 . \backslash \mathrm{PG}=\mathrm{CS} \quad[\mathrm{SG}(\mathrm{N} 101$ )$, \mathrm{X}(\mathrm{C} 12 \mathrm{H} 16)] \backslash \backslash \mathrm{Q}$

\section{5-carboxy-TMIO•(24)}

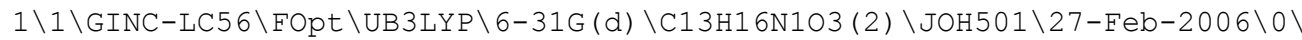
\\# UB3LYP/6-31G* OPT FREQ=NORAMAN MAXDISK=134217728 \\ctmio $2 \mathrm{~s} \backslash \backslash 0,2 \backslash 0,3$ $.5900321109,0.6674141869,0 . \backslash \mathrm{N}, 2.3596694848,0.3378055878,0 . \backslash \mathrm{C}, 1.9235466$ $458,-1.0876967636,0 . \backslash C, 1.2660515236,1.3517449981,0 . \backslash C, 0.420797207,-0.9$ 
$118670152,0 . \backslash C, 0.0557764666,0.4417539608,0 . \backslash C,-0.554699225,-1.90800140$ $53,0 . \backslash \mathrm{C},-1.2840939033,0.8075207817,0 . \backslash \mathrm{H},-0.2755335333,-2.9583137019,0$. $\backslash \mathrm{H},-1.5973305032,1.8469802159,0 . \backslash \mathrm{C},-1.901224334,-1.5459238372,0 . \backslash \mathrm{C},-2$. $2687451835,-0.1914879337,0 . \backslash \mathrm{H},-2.6722702593,-2.3080371105,0 . \backslash \mathrm{C},-3.6906$ $06606,0.241153948,0 . \backslash 0,-4.0665005426,1.396800117,0 . \backslash 0,-4.5648584779,-0$ $.7986986914,0 . \backslash \mathrm{H},-5.4522138468,-0.3946079754,0 . \backslash \mathrm{C}, 1.3767478747,2.22135$ $68571,-1.2653399541 \backslash \mathrm{C}, 1.3767478747,2.2213568571,1.2653399541 \backslash \mathrm{C}, 2.45646$ $78292,-1.7842609218,-1.2656556718 \backslash \mathrm{C}, 2.4564678292,-1.7842609218,1.26565$ $56718 \backslash \mathrm{H}, 3.5472704102,-1.7023533411,-1.2887829709 \backslash \mathrm{H}, 3.5472704102,-1.702$ $3533411,1.2887829709 \backslash \mathrm{H}, 2.0520963069,-1.3187022603,-2.1698338616 \backslash \mathrm{H}, 2.05$ $20963069,-1.3187022603,2.1698338616 \backslash \mathrm{H}, 2.1809192738,-2.8443363009,-1.26$ $77612742 \backslash \mathrm{H}, 2.1809192738,-2.8443363009,1.2677612742 \backslash \mathrm{H}, 2.3581189554,2.70$ $46980352,-1.2870383179 \backslash \mathrm{H}, 2.3581189554,2.7046980352,1.2870383179 \backslash \mathrm{H}, 0.60$ $09948696,2.9944373621,-1.2694534236 \backslash \mathrm{H}, 0.6009948696,2.9944373621,1.2694$ $534236 \backslash \mathrm{H}, 1.2660367004,1.6146979705,-2.1695826133 \backslash \mathrm{H}, 1.2660367004,1.6146$ $979705,2.1695826133 \backslash \backslash$ Version=IA32L-G03RevC.02 State=2-A" $\backslash H F=-785.41165$ $62 \backslash \mathrm{S} 2=0.753783 \backslash \mathrm{S} 2-1=0 . \backslash \mathrm{S} 2 \mathrm{~A}=0.750011 \backslash \mathrm{RMSD}=9.949 \mathrm{e}-09 \backslash \mathrm{RMSF}=7.999 \mathrm{e}-06 \backslash \mathrm{Dipo}$ $l e=-0.6150363,-0.8933109,0 . \backslash P G=C S \quad[S G(C 9 H 4 N 1 O 3), X(C 4 H 12)] \backslash \backslash @$

\section{5-carboxy-TMIO+}

$1 \backslash 1 \backslash G I N C-L C 74 \backslash F O p t \backslash U B 3 L Y P \backslash 6-31 G(d) \backslash C 13 H 16 N 103(1+) \backslash J O H 501 \backslash 10-M a r-2006 \backslash 0$ $\backslash \backslash \#$ UB3LYP/6-31G* OPT FREQ=NORAMAN MAXDISK=268435456\\Ctmio+2s $\backslash \backslash 1,1 \backslash 0$ $, 3.5671799831,0.2878222736,0 . \backslash \mathrm{N}, 2.3766384828,0.2886862345,0 .{ }^{\mathrm{C}}, 1.55481$ $94485,1.571562363,0 . \backslash \mathrm{C}, 1.5505965404,-0.9891663745,0 . \backslash \mathrm{C}, 0.1599087866,0$. $9949186272,0 . \backslash \mathrm{C}, 0.1586343795,-0.4051535582,0 . \backslash \mathrm{C},-1.0372665709,1.707015$ $6119,0 . \backslash \mathrm{C},-1.0362084859,-1.1127126233,0 . \backslash \mathrm{H},-1.0463014924,2.7931027284$, $0 . \backslash \mathrm{H},-1.0756474894,-2.1977338986,0 . \backslash \mathrm{C},-2.2410399789,1.0004164745,0 . \backslash \mathrm{C}$, $-2.2418909941,-0.401218794,0 . \backslash \mathrm{H},-3.1843922594,1.5341610697,0 . \backslash \mathrm{C},-3.505$ $9725407,-1.2058721483,0.10,-3.5255319456,-2.417188735,0.10,-4.60878024$ $44,-0.4335651352,0 . \backslash \mathrm{H},-5.3802981772,-1.032065153,0 . \backslash \mathrm{C}, 1.9261302454,-1$. $7753880648,1.2770757136 \backslash \mathrm{C}, 1.9261302454,-1.7753880648,-1.2770757136 \backslash \mathrm{C}, 1$ $.9346484955,2.354808838,1.2773440552 \backslash C, 1.9346484955,2.354808838,-1.277$ $3440552 \backslash \mathrm{H}, 2.9937510077,2.6256302113,1.2645624579 \backslash \mathrm{H}, 2.9937510077,2.6256$ $302113,-1.2645624579 \backslash \mathrm{H}, 1.7068208338,1.7845747921,2.181765972 \backslash \mathrm{H}, 1.70682$ $08338,1.7845747921,-2.181765972 \backslash \mathrm{H}, 1.337316652,3.2707111727,1.292460910$ $4 \backslash \mathrm{H}, 1.337316652,3.2707111727,-1.2924609104 \backslash \mathrm{H}, 2.9824793455,-2.056562010$ $7,1.262534982 \backslash \mathrm{H}, 2.9824793455,-2.0565620107,-1.262534982 \backslash \mathrm{H}, 1.3189030753$ $,-2.6848189456,1.2927527349 \backslash \mathrm{H}, 1.3189030753,-2.6848189456,-1.2927527349$ $\backslash \mathrm{H}, 1.704928733,-1.2028364014,2.181690485 \backslash \mathrm{H}, 1.704928733,-1.2028364014,-$ $2.181690485 \backslash \backslash$ Version=IA32L-G03RevC.02\State=1-A $\backslash$ SHF $=-785.1562727 \backslash S 2=0$. $\backslash \mathrm{S} 2-1=0 . \backslash \mathrm{S} 2 \mathrm{~A}=0 . \backslash \mathrm{RMSD}=6.515 \mathrm{e}-09 \backslash \mathrm{RMSF}=9.880 \mathrm{e}-05 \backslash \mathrm{Dipole}=2.0212135,1.10830$ $87,0 . \backslash P G=C S \quad[S G(C 9 H 4 N 103), X(C 4 H 12)] \backslash \backslash @$

\section{5-carboxy-TMIO-}

$1 \backslash 1 \backslash$ GINC-LC1 45 FOpt \UB3LYP \6-31G (d) \C13H16N1O3 (1-) \JOH501\02-Mar-2006

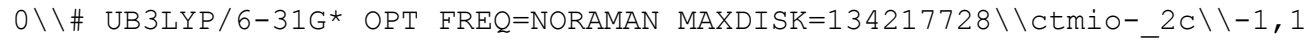
$\backslash 0,-3.5777888801,0.719332154,-0.1437205901 \backslash \mathrm{N},-2.3035026871,0 . \overline{3} 46858869$ $,-0.418420978 \backslash \mathrm{C},-1.9311756128,-1.0396670576,-0.0275917812 \backslash \mathrm{C},-1.2415997$ $695,1.3151832859,-0.0264060641 \backslash C,-0.4259019654,-0.9208194097,-0.086498$ $5953 \backslash C,-0.0257747831,0.4251853985,-0.0770048365 \backslash C, 0.5397448238,-1.9378$ $594737,-0.0451758616 \backslash \mathrm{C}, 1.3174957541,0.7705345667,-0.0521633603 \backslash \mathrm{H}, 0.238$ $9067946,-2.9835136751,-0.0341481955 \backslash \mathrm{H}, 1.6272992768,1.8104951118,-0.041$ $7770934 \backslash C, 1.8895649835,-1.6021943587,-0.019969994 \backslash C, 2.2981347023,-0.25$ $44686016,-0.0241651285 \backslash \mathrm{H}, 2.6573212646,-2.3701444751,0.0062668185 \backslash \mathrm{C}, 3.7$ $336846848,0.0266425801,0.0071717721 \backslash 0,4.6442991019,-0.7959121883,0.021$ $3143514 \backslash 0,4.0360388563,1.369397817,0.0172738837 \backslash \mathrm{H}, 5.0094087605,1.38377$ $65868,0.0355920996 \backslash \mathrm{C},-1.4990607617,1.9164758984,1.3780828231 \backslash \mathrm{C},-1.2525$ $274605,2.4421842499,-1.0736573913 \backslash \mathrm{C},-2.4649877679,-1.4420483165,1.3766$ $140931 \backslash \mathrm{C},-2.5482025437,-1.9874706204,-1.0745892492 \backslash \mathrm{H},-3.4816919347,-1$. $0382970762,1.4355024807 \backslash \mathrm{H},-3.6298731775,-1.8143341648,-1.0815841022 \backslash \mathrm{H}$, $-1.8659491121,-0.9986743908,2.1795963695 \backslash \mathrm{H},-2.1574333844,-1.752228319$, $-2.0703192641 \backslash \mathrm{H},-2.4833668618,-2.5296862921,1.5248790509 \backslash \mathrm{H},-2.35321305$ $19,-3.0456390839,-0.8510355187 \backslash \mathrm{H},-2.5867349158,2.0583534033,1.42994547$ 
$54 \backslash \mathrm{H},-2.2660030381,2.8575564944,-1.0955843336 \backslash \mathrm{H},-0.9821520546,2.872107$ $7279,1.5334129792 \backslash \mathrm{H},-0.5365474821,3.2413629804,-0.8384105151 \backslash \mathrm{H},-1.1948$ $402517,1.2306079405,2.1761193491 \backslash \mathrm{H},-1.0273703499,2.0376340397,-2.06632$ $84708 \backslash \backslash$ Version=IA32L-G03RevC.02 $\backslash$ State $=1-A \backslash H F=-785.4130243 \backslash \mathrm{S} 2=0 . \backslash \mathrm{S} 2-1=0$ $. \backslash \mathrm{S} 2 \mathrm{~A}=0 . \backslash \mathrm{RMSD}=4.688 \mathrm{e}-09 \backslash \mathrm{RMSF}=1.417 \mathrm{e}-05 \backslash \mathrm{Dipole}=2.0767355,-0.1906292,0.2$ $316813 \backslash P G=C 01 \quad[X(C 13 H 16 \mathrm{~N} 103)] \backslash \backslash @$

\section{5-amino-TMIO•(25)}

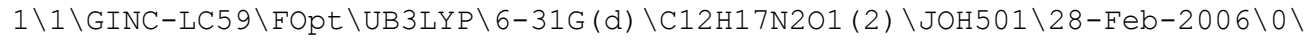
\\# UB3LYP/6-31G* OPT FREQ=NORAMAN MAXDISK=134217728\\ntmio $1 \backslash \backslash 0,2 \backslash \mathrm{N},-0$ $.8899144218,-1.5985026031,0.0098194426 \backslash \mathrm{C}, 0.6013834377,-1.6 \overline{1} 36800846,0$. $018876285 \backslash \mathrm{C}, 0.8833055148,-0.1266443285,0.0075173825 \backslash \mathrm{C},-0.2928762406,0$. $631561867,-0.0040949223 \backslash \mathrm{C},-0.2529419804,2.0212415923,-0.0178249773 \backslash \mathrm{C}, 0$ $.988499235,2.6816557621,-0.0189219634 \backslash C, 2.1164293205,0.5222503454,0.00$ $55603475 \backslash \mathrm{C}, 2.1688771546,1.9146077086,-0.0081982449 \backslash \mathrm{C},-1.5247523031,-0$. $2494483522,-0.0019734495 \backslash \mathrm{H},-1.1715907948,2.6046926482,-0.0229582819 \backslash \mathrm{N}$, $1.0498222299,4.0777622512,0.029371088 \backslash \mathrm{H}, 3.0430592044,-0.0468270622,0.0$ $117807441 \backslash \mathrm{H}, 3.131683384,2.4206345371,-0.0041676704 \backslash 0,-1.5801153465,-2$. $6698983297,0.0139280892 \backslash \mathrm{C},-2.3844489897,-0.1062477908,-1.2709772286 \backslash \mathrm{C}$, $-2.3925851527,-0.0870952156,1.2590914313 \backslash \mathrm{C}, 1.0927142658,-2.3256784958$, $1.2926606191 \backslash \mathrm{C}, 1.1075982872,-2.3487648504,-1.2356831544 \backslash \mathrm{H},-2.903202607$ $3,0.8820395307,1.2544381464 \backslash \mathrm{H},-3.1436259541,-0.8824922958,1.2870534957$ $\backslash \mathrm{H},-1.7811306322,-0.1508572209,2.1644739051 \backslash \mathrm{H},-2.8960714049,0.86233467$ $03,-1.2836617112 \backslash \mathrm{H},-1.7672290916,-0.1835286827,-2.1715187726 \backslash \mathrm{H},-3.1348$ $437517,-0.9024298415,-1.2920171211 \backslash \mathrm{H}, 2.1870844787,-2.3770285033,1.3060$ $655146 \backslash \mathrm{H}, 0.7610999591,-1.7935169495,2.1896446036 \backslash \mathrm{H}, 0.6917357395,-3.343$ $6302727,1.3209546648 \backslash \mathrm{H}, 2.2020488065,-2.400171019,-1.2352892695 \backslash \mathrm{H}, 0.707$ $0267057,-3.3671453381,-1.2499253058 \backslash \mathrm{H}, 0.786242691,-1.8334852035,-2.146$ $2390415 \backslash \mathrm{H}, 1.9063676376,4.4796875922,-0.3310799957 \backslash \mathrm{H}, 0.235698452,4.5535$ $435672,-0.3395050831 \backslash \backslash$ Version $=I A 32 \mathrm{~L}-\mathrm{G} 03$ RevC $.02 \backslash$ State $=2-A \backslash H F=-652.19152$ $66 \backslash S 2=0.753793 \backslash S 2-1=0 . \backslash S 2 A=0.750011 \backslash R M S D=5.343 e-09 \backslash R M S F=2.935 e-05 \backslash D i p o$ $l e=0.6781939,1.532314,-0.4438283 \backslash P G=C 01 \quad[X(C 12 \mathrm{H} 17 \mathrm{~N} 201)] \backslash \backslash @$

\section{5-amino-TMIO+}

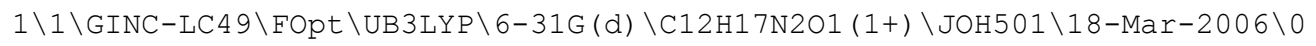
$\backslash \backslash \#$ UB3LYP/6-31G* OPT FREQ=NORAMAN MAXDISK=268435456\\ntmio+ $3 \backslash \backslash 1,1 \backslash \mathrm{N}$, $1.8096003321,0.2741197492,-0.0157100653 \backslash \mathrm{C}, 1.2910582497,-1.1658131501,-$ $0.0112580828 \backslash C,-0.1916363896,-0.8666658047,0.002080121 \backslash C,-0.4913908214$ $, 0.4997238375,0.0052433211 \backslash \mathrm{C},-1.7983254584,0.960378453,0.0167480228 \backslash \mathrm{C}$, $-2.8595074505,0.0251082331,0.0241834656 \backslash \mathrm{C},-1.2359223943,-1.7983089947$, $0.0104962166 \backslash \mathrm{C},-2.5496758781,-1.3604562214,0.0213354074 \backslash \mathrm{C}, 0.7470296363$ $, 1.3625929355,-0.0054049719 \backslash \mathrm{H},-2.0193158456,2.02435409,0.0177005932 \backslash \mathrm{N}$, $-4.1558516638,0.4397383543,0.0185628862 \backslash \mathrm{H},-1.0307803859,-2.8651865227$, $0.0087559794 \backslash \mathrm{H},-3.3597708122,-2.0839747369,0.0260591004 \backslash 0,2.9772370399$ $, 0.5285730338,-0.0269041231 \backslash \mathrm{C}, 0.9623945588,2.2083210092,1.2673720069 \backslash \mathrm{C}$ $, 0.9389697431,2.2096572064,-1.2810519685 \backslash C, 1.8114875401,-1.8540606114$, $-1.2880562618 \backslash \mathrm{C}, 1.8347499026,-1.8545414301,1.2555626285 \backslash \mathrm{H}, 0.1754417155$ $, 2.9923265453,-1.2870692904 \backslash \mathrm{H}, 1.9238611161,2.6848197161,-1.2852469893 \backslash$ $\mathrm{H}, 0.8164209387,1.6060180355,-2.1843432539 \backslash \mathrm{H}, 0.1995375676,2.9913966575$, $1.2879347224 \backslash \mathrm{H}, 0.8561681819,1.6038665182,2.1721978598 \backslash \mathrm{H}, 1.9474006626,2$ $.6830877801,1.2541198421 \backslash \mathrm{H}, 1.4059081379,-2.8702792937,-1.309505562 \backslash \mathrm{H}, 1$ $.4729475392,-1.3362234837,-2.1897157121 \backslash \mathrm{H}, 2.9031589115,-1.9192166666,-$ $1.2874441755 \backslash \mathrm{H}, 1.4296458913,-2.8707744442,1.2840669818 \backslash \mathrm{H}, 2.9262260776$, $-1.9197246127,1.2349378768 \backslash \mathrm{H}, 1.5128568999,-1.3370183061,2.1634867104 \backslash \mathrm{H}$ $,-4.9140958119,-0.2216147971,0.0936773701 \backslash \mathrm{H},-4.3951352114,1.4169397532$ , $0.0921517558 \backslash \backslash$ Version=IA32L-G03RevC.02 $\backslash$ State $=1-A \backslash H F=-651.9486289 \backslash \mathrm{S} 2=0$ $. \backslash \mathrm{S} 2-1=0 . \backslash \mathrm{S} 2 \mathrm{~A}=0 . \backslash \mathrm{RMSD}=4.350 \mathrm{e}-09 \backslash \mathrm{RMSF}=1.975 \mathrm{e}-05 \backslash \mathrm{Dipole}=-0.587576,0.2676$ $043,0.0940653 \backslash P G=C 01 \quad[X(C 12 \mathrm{H} 17 \mathrm{~N} 201)] \backslash \backslash @$

\section{5-amino-TMIO-}

$1 \backslash 1 \backslash G I N C-A C 28 \backslash F O p t \backslash U B 3 L Y P \backslash 6-31 G(d) \backslash C 12 H 17 N 2 O 1$ (1-) \JOH501\01-Mar-2006\0 
\\\# UB3LYP/6-31G* OPT FREQ=NORAMAN MAXDISK=134217728\\ntmio- $1 \backslash \backslash-1,1 \backslash \mathrm{N}$ $, 1.7531052019,0.2576265168,0.5022910141 \backslash \mathrm{C}, 1.280646606,-1.091 \overline{5} 962134,0$. $0394360994 \backslash \mathrm{C},-0.2155895657,-0.8803924643,0.0442498597 \backslash \mathrm{C},-0.5072028086$, $0.4933575252,0.0409490079 \backslash \mathrm{C},-1.8250379796,0.9407677475,0.0042676251 \backslash \mathrm{C}$, $-2.8797946671,0.0107576625,-0.0246903005 \backslash C,-1.2571269209,-1.8025080221$ $, 0.0106361413 \backslash \mathrm{C},-2.5876872821,-1.3585355944,-0.0224359522 \backslash \mathrm{C}, 0.77458869$ $82,1.2941618383,0.0315377862 \backslash \mathrm{H},-2.0485848529,2.0075139434,-0.003974802$ $3 \backslash \mathrm{N},-4.2280715673,0.4614377193,-0.1115847132 \backslash \mathrm{H},-1.0498808488,-2.871372$ $4836,0.0119457658 \backslash \mathrm{H},-3.4051316508,-2.0792564414,-0.053013557 \backslash 0,3.06575$ $28848,0.5375845258,0.1966805631 \backslash C, 0.8288249055,2.4367094736,1.06012619$ $9 \backslash C, 1.1375207995,1.8542974569,-1.3675980764 \backslash C, 1.8496734958,-1.46274920$ $01,-1.3529039525 \backslash \mathrm{C}, 1.7898139785,-2.1054074647,1.0778947964 \backslash \mathrm{H}, 0.7681544$ $854,2.8757998935,-1.5308984254 \backslash \mathrm{H}, 2.2375881935,1.8325213055,-1.37821503$ $86 \backslash \mathrm{H}, 0.7581573741,1.2213164149,-2.1773294279 \backslash \mathrm{H}, 0.1827012505,3.28526963$ $07,0.791050258 \backslash \mathrm{H}, 0.5456875895,2.0657092288,2.0514283815 \backslash \mathrm{H}, 1.8702900256$ $, 2.7742113937,1.1075401414 \backslash \mathrm{H}, 1.9312515071,-2.5471766294,-1.5082988199 \backslash$ $\mathrm{H}, 1.2490452229,-1.0464954196,-2.1692142601 \backslash \mathrm{H}, 2.8452181095,-0.993414808$ $9,-1.3620421416 \backslash \mathrm{H}, 1.5398693343,-3.1443071808,0.8168473091 \backslash \mathrm{H}, 2.87912912$ $68,-1.9961859929,1.1276975777 \backslash \mathrm{H}, 1.378286333,-1.8719978239,2.0660956201$ $\backslash \mathrm{H},-4.8710658325,-0.1907247356,0.3279676642 \backslash \mathrm{H},-4.3437494451,1.37128737$ $75,0.3252097442 \backslash \backslash$ Version=IA64L-G03RevC.02\State $=1-\mathrm{A} \backslash \mathrm{HF}=-652.1780357 \backslash \mathrm{S} 2$ $=0 . \backslash \mathrm{S} 2-1=0 . \backslash \mathrm{S} 2 \mathrm{~A}=0 . \backslash \mathrm{RMSD}=6.789 \mathrm{e}-09 \backslash \mathrm{RMSF}=5.746 \mathrm{e}-06 \backslash \mathrm{Dipole}=-3.3996729,-0$. $3968302,0.2197464 \backslash \mathrm{PG}=\mathrm{C} 01 \quad[\mathrm{X}(\mathrm{C} 12 \mathrm{H} 17 \mathrm{~N} 2 \mathrm{O} 1)] \backslash \backslash @$

\section{5-methoxy-TMIO•(26)}

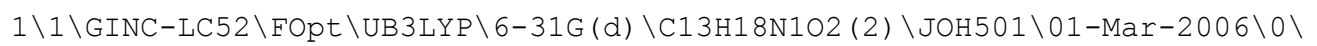

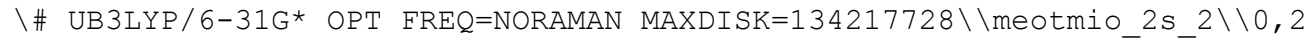
$\backslash 0,3.2480505761,0.9497056561,0 . \backslash \mathrm{N}, 2.0615187213,0.4848143163, \overline{0} . \backslash \overline{\mathrm{C}}, 0.864$ $0302552,1.3727753266,0 . \backslash \mathrm{C}, 1.7813655289,-0.9800299322,0 . \backslash \mathrm{C}, 0.2679640078$ $,-0.9623403047,0 . \backslash C,-0.2410987348,0.3369903861,0 . \backslash C,-0.6099282809,-2.0$ $487756712,0 . \backslash C,-1.6161761643,0.5790025992,0 . \backslash C,-1.9820940688,-1.825531$ $2167,0 . \backslash C,-2.4903567411,-0.5151547274,0 . \backslash 0,-3.8523738426,-0.4167991445$ $, 0 . \backslash \mathrm{C},-4.4339854339,0.8767129151,0 . \backslash \mathrm{H},-5.5140840164,0.7192436285,0 . \backslash \mathrm{H}$, $-2.6878275605,-2.6501450349,0 . \backslash \mathrm{H},-1.9913718216,1.5964233049,0 . \backslash \mathrm{H},-0.23$ $26930145,-3.0684410922,0 . \backslash \mathrm{H},-4.1513852796,1.4474590922,-0.8947224811 \backslash \mathrm{H}$ $,-4.1513852796,1.4474590922,0.8947224811 \backslash \mathrm{C}, 0.8826672675,2.2502277829,1$ $.2649282639 \backslash C, 0.8826672675,2.2502277829,-1.2649282639 \backslash C, 2.3905101165,-$ $1.6129171912,1.2644315541 \backslash \mathrm{C}, 2.3905101165,-1.6129171912,-1.2644315541 \backslash \mathrm{H}$ $, 0.0316554787,2.9401581581,1.2686736199 \backslash \mathrm{H}, 0.0316554787,2.9401581581,-1$ $.2686736199 \backslash \mathrm{H}, 1.8089839821,2.8322995874,1.2882781627 \backslash \mathrm{H}, 1.8089839821,2$. $8322995874,-1.2882781627 \backslash \mathrm{H}, 0.8335146408,1.6348179727,2.1686336826 \backslash \mathrm{H}, 0$. $8335146408,1.6348179727,-2.1686336826 \backslash \mathrm{H}, 3.4676119195,-1.4205061073,1.2$ $860313531 \backslash \mathrm{H}, 3.4676119195,-1.4205061073,-1.2860313531 \backslash \mathrm{H}, 1.941170951,-1$. $190158933,2.1684640874 \backslash \mathrm{H}, 1.941170951,-1.190158933,-2.1684640874 \backslash \mathrm{H}, 2.22$ $51886473,-2.6958980015,1.2699915667 \backslash \mathrm{H}, 2.2251886473,-2.6958980015,-1.26$ 99915667 \Version=IA32L-G03RevC.02 \State $=2-A " \backslash H F=-711.3607472 \backslash S 2=0.753$ $789 \backslash \mathrm{S} 2-1=0 . \backslash \mathrm{S} 2 \mathrm{~A}=0.750011 \backslash \mathrm{RMSD}=4.076 \mathrm{e}-09 \backslash \mathrm{RMSF}=6.094 \mathrm{e}-06 \backslash \mathrm{Dipole}=-1.24033$ $79,0.0708254,0 . \backslash \mathrm{PG}=\mathrm{CS} \quad[\mathrm{SG}(\mathrm{C} 9 \mathrm{H} 4 \mathrm{N1O}), \mathrm{X}(\mathrm{C} 4 \mathrm{H} 14)] \backslash \backslash \mathrm{e}$

\section{5-methoxy-TMIO+}

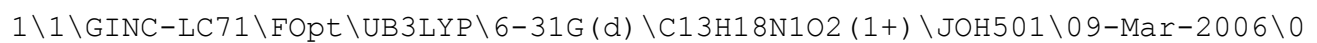

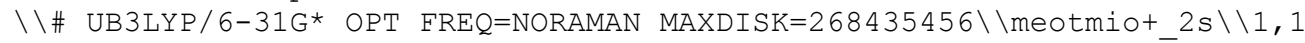
$\backslash 0,-3.3819530893,-0.056867773,0 . \backslash N,-2.1922368083,-0.0929283807,0 . \backslash \mathrm{C},-1$ $.3343660072,1.1660240864,0 . \backslash C,-1.4089571588,-1.4028573741,0 . \backslash C,-0.0020$ $744117,-0.8553950039,0 . \backslash \mathrm{C}, 0.04119584,0.5451668786,0 . \backslash \mathrm{C}, 1.1855064306,-1$ $.5814791847,0 . \backslash C, 1.2443648376,1.2299751571,0 . \backslash C, 2.4087039977,-0.910384$ $8725,0 . \backslash \mathrm{C}, 2.4465068815,0.4961366523,0 . \backslash 0,3.5711262626,1.235623897,0 . \backslash \mathrm{C}$ $, 4.8428375889,0.5789931338,0 . \backslash \mathrm{H}, 5.5823104949,1.3792868188,0 . \backslash \mathrm{H}, 3.32615$ $94298,-1.4859768559,0 . \backslash \mathrm{H}, 1.302017078,2.3138904408,0 . \backslash \mathrm{H}, 1.1778749155,-2$ $.6680429464,0 . \backslash \mathrm{H}, 4.9676786843,-0.0365848701,-0.8979563701 \backslash \mathrm{H}, 4.96767868$ $43,-0.0365848701,0.8979563701 \backslash \mathrm{C},-1.6910303774,1.9599704505,-1.27635923$ $93 \backslash \mathrm{C},-1.6910303774,1.9599704505,1.2763592393 \backslash \mathrm{C},-1.8149183718,-2.174557$ $3483,-1.2749908451 \backslash \mathrm{C},-1.8149183718,-2.1745573483,1.2749908451 \backslash \mathrm{H},-1.066$ 
$7558357,2.8575654052,-1.2918267637 \backslash \mathrm{H},-1.0667558357,2.8575654052,1.2918$ $267637 \backslash \mathrm{H},-2.7417408838,2.2623399577,-1.2653734457 \backslash \mathrm{H},-2.7417408838,2.26$ $23399577,1.2653734457 \backslash \mathrm{H},-1.4792044031,1.3827402947,-2.1802823578 \backslash \mathrm{H},-1$. $4792044031,1.3827402947,2.1802823578 \backslash \mathrm{H},-2.8790812433,-2.4256717924,-1$. $2610060799 \backslash \mathrm{H},-2.8790812433,-2.4256717924,1.2610060799 \backslash \mathrm{H},-1.5778128778$, $-1.6079948805,-2.1794351939 \backslash \mathrm{H},-1.5778128778,-1.6079948805,2.1794351939$ $\backslash \mathrm{H},-1.2365897646,-3.102765039,-1.2950848634 \backslash \mathrm{H},-1.2365897646,-3.1027650$ $39,1.2950848634 \backslash \backslash$ Version=IA32L-G03RevC.02\State=1-A' $\backslash H F=-711.1139801 \backslash S$ $2=0 . \backslash S 2-1=0 . \backslash S 2 A=0 . \backslash R M S D=9.476 e-09 \backslash R M S F=8.112 e-05 \backslash D i p o l e=-0.896004,-0$. $4445228,0 . \backslash \mathrm{PG}=\mathrm{CS} \quad[\mathrm{SG}(\mathrm{C} 9 \mathrm{H} 4 \mathrm{~N} 1 \mathrm{O} 2), \mathrm{X}(\mathrm{C} 4 \mathrm{H} 14)] \backslash \backslash \mathrm{C}$

\section{5-methoxy-TMIO-}

$1 \backslash 1 \backslash G I N C-L C 38 \backslash F O p t \backslash U B 3 L Y P \backslash 6-31 G(d) \backslash C 13 H 18 N 102$ (1-) \JOH501\01-Mar-2006\0 $\backslash \backslash \#$ UB3LYP/6-31G* OPT FREQ=NORAMAN MAXDISK=134217728\\meotmio- $3 \backslash \backslash-1,1$ $\backslash \mathrm{N},-0.8804411473,-1.8987401943,0.3777082056 \backslash \mathrm{C}, 0.6213024833,-1 . \overline{8} 6023151$ $42,0.3781207244 \backslash C, 0.8645426873,-0.3692959543,0.3672409652 \backslash C,-0.2914244$ $889,0.3026734805,-0.0491173609 \backslash \mathrm{C},-0.3057038739,1.6928635669,-0.1922051$ $04 \backslash C, 0.863181781,2.41347381,0.0922691446 \backslash C, 2.0214245647,0.357857189,0$. $6472155907 \backslash C, 2.0220604725,1.7503811253,0.5100093454 \backslash C,-1.3905673956,-0$ $.690021169,-0.3505508806 \backslash \mathrm{H},-1.2109002243,2.1926757386,-0.5220658085 \backslash 0$, $0.9725112079,3.7916429623,-0.0140493794 \backslash \mathrm{H}, 2.9296253409,-0.1491042546,0$ $.9681940003 \backslash \mathrm{H}, 2.9103990471,2.3414792573,0.7179663326 \backslash 0,-1.4199223857,-$ $3.0892944644,-0.0529269742 \backslash C,-1.5801096087,-0.9523635132,-1.866740801 \backslash$ $C,-2.754205528,-0.3513811078,0.2761377465 \backslash C, 1.069921071,-2.5746443058$, $1.6640559067 \backslash \mathrm{C}, 1.2233058048,-2.5916946204,-0.8476908342 \backslash \mathrm{H},-3.254568197$ $7,0.4939438825,-0.2189892475 \backslash \mathrm{H},-3.3792477945,-1.2466900184,0.184127166$ $2 \backslash \mathrm{H},-2.6343408204,-0.1274193345,1.3418320829 \backslash \mathrm{H},-2.3478575548,-0.311820$ $7983,-2.321551018 \backslash \mathrm{H},-0.6488434825,-0.8202063112,-2.4286064923 \backslash \mathrm{H},-1.880$ $22041,-2.0099913474,-1.9116710731 \backslash \mathrm{H}, 2.1637002326,-2.6535476461,1.74850$ $67817 \backslash \mathrm{H}, 0.676170182,-2.0516705557,2.5425203713 \backslash \mathrm{H}, 0.6344283377,-3.58001$ $51599,1.6418460538 \backslash \mathrm{H}, 2.2294011769,-2.9899746556,-0.658798874 \backslash \mathrm{H}, 0.51159$ $39406,-3.4089375837,-1.0400421714 \backslash \mathrm{H}, 1.2735275432,-1.9448974703,-1.7307$ $390194 \backslash \mathrm{C},-0.1695387517,4.5049814474,-0.4292610227 \backslash \mathrm{H}, 0.1178888759,5.560$ $7439025,-0.4449838697 \backslash \mathrm{H},-0.4994553905,4.2085849075,-1.4360341072 \backslash \mathrm{H},-1$. $0140586562,4.3736502177,0.2634417613 \backslash \backslash$ Version=IA32L-G03RevC.02\State=1 $-\mathrm{A} \backslash \mathrm{HF}=-711.3502242 \backslash \mathrm{S} 2=0 . \backslash \mathrm{S} 2-1=0 . \backslash \mathrm{S} 2 \mathrm{~A}=0 . \backslash \mathrm{RMSD}=9.779 \mathrm{e}-09 \backslash \mathrm{RMSF}=2.610 \mathrm{e}-05 \backslash$ Dipole $=1.0045194,3.4150794,-0.1969767 \backslash P G=C 01 \quad[X(C 13 H 18 N 102)] \backslash \backslash @$

\section{5-hydroxy-TMIO• $(27)$}

$1 \backslash 1 \backslash G I N C-L C 114 \backslash$ FOpt \UB3LYP \6-31G (d) \C12H16N1O2 (2) \JOH501\28-Feb-2006

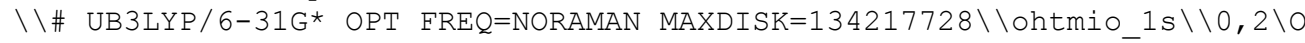
$, 3.0547598101,0.4996395769,0 . \backslash N, 1.8050783539,0.2506505939,0 . \backslash \mathrm{C}, 1.27405$ $4548,-1.1429417852,0 . \backslash C, 0.7819865716,1.3355667895,0 . \backslash C,-0.4882554899,0$ $.5114060059,0 . \backslash C,-0.2136708229,-0.8627378512,0 . \backslash C,-1.7957286122,0.9831$ $79849,0 . \backslash C,-1.2632027885,-1.7772029494,0 . \backslash C,-2.5816263515,-1.316989951$ $8,0 . \backslash \mathrm{C},-2.8468502243,0.0584786605,0 . \backslash 0,-4.1192390057,0.5613710751,0 . \backslash \mathrm{H}$ $,-4.7500329003,-0.1751873202,0 . \backslash \mathrm{H},-2.028399694,2.0438318927,0 . \backslash \mathrm{H},-3.40$ $69224305,-2.0268350745,0 . \backslash \mathrm{H},-1.0701132916,-2.8470606541,0 . \backslash \mathrm{C}, 1.7640186$ $018,-1.8718308142,1.2644962615 \backslash \mathrm{C}, 1.7640186018,-1.8718308142,-1.2644962$ $615 \backslash \mathrm{C}, 0.9530330247,2.1957054546,1.2651485596 \backslash \mathrm{C}, 0.9530330247,2.19570545$ $46,-1.2651485596 \backslash \mathrm{H}, 0.2346673087,3.0225374486,1.268804416 \backslash \mathrm{H}, 0.234667308$ $7,3.0225374486,-1.268804416 \backslash \mathrm{H}, 1.9666428092,2.6072883868,1.2883276139 \backslash \mathrm{H}$ $, 1.9666428092,2.6072883868,-1.2883276139 \backslash \mathrm{H}, 0.7974301664,1.5980577519,2$ $.1687535994 \backslash \mathrm{H}, 0.7974301664,1.5980577519,-2.1687535994 \backslash \mathrm{H}, 2.8581252473,-$ $1.8676699998,1.2867331789 \backslash \mathrm{H}, 2.8581252473,-1.8676699998,-1.2867331789 \backslash \mathrm{H}$ $, 1.3937721697,-1.3781579786,2.1684154624 \backslash \mathrm{H}, 1.3937721697,-1.3781579786$, $-2.1684154624 \backslash \mathrm{H}, 1.4148087519,-2.910273862,1.2695030042 \backslash \mathrm{H}, 1.4148087519$, $-2.910273862,-1.2695030042 \backslash \backslash$ Version=IA32L-G03RevC.02 $\backslash$ State $=2-A " \backslash H F=-67$ $2.0542768 \backslash \mathrm{S} 2=0.75379 \backslash \mathrm{S} 2-1=0 . \backslash \mathrm{S} 2 \mathrm{~A}=0.750011 \backslash \mathrm{RMSD}=4.346 \mathrm{e}-09 \backslash \mathrm{RMSF}=1.745 e-0$ $5 \backslash$ Dipole $=-1.3326066,-0.6990021,0 . \backslash \mathrm{PG}=\mathrm{CS}[\mathrm{SG}(\mathrm{C} 8 \mathrm{H} 4 \mathrm{~N} 102), \mathrm{X}(\mathrm{C} 4 \mathrm{H} 12)] \backslash \backslash \mathrm{Q}$ 


\section{5-hydroxy-TMIO+}

$1 \backslash 1 \backslash G I N C-L C 38 \backslash F O p t \backslash U B 3 L Y P \backslash 6-31 G(d) \backslash C 12 H 16 N 1 O 2(1+) \backslash J O H 501 \backslash 09-M a r-2006 \backslash 0$

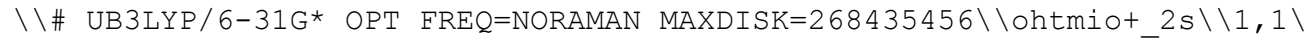
$0,-2.9806704069,0.4953885002,0 . \backslash N,-1.8143613571,0.2581610758, \overline{0} . \backslash \mathrm{C},-1.2$ $632850084,-1.1635721462,0 . \backslash \mathrm{C},-0.7547575177,1.351460461,0 . \backslash \mathrm{C}, 0.21624695$ $3,-0.8647825545,0 . \backslash C, 0.4961859752,0.5063567034,0 . \backslash C, 1.2625356637,-1.78$ $59079238,0 . \backslash C, 1.7998005506,0.9809267601,0 . \backslash C, 2.5774598123,-1.327050763$ $6,0 . \backslash \mathrm{C}, 2.8514629132,0.0512747501,0 . \backslash \mathrm{H}, 1.0704859753,-2.8551453531,0 . \backslash \mathrm{H}$, $3.3974501384,-2.0405812123,0 . \backslash \mathrm{H}, 2.0381739931,2.0397265892,0 . \backslash 0,4.10392$ $46618,0.5573339964,0 . \backslash \mathrm{H}, 4.7638318627,-0.1551300506,0 . \backslash \mathrm{C},-1.794116811,-$ $1.8551135723,1.2755929422 \backslash \mathrm{C},-1.794116811,-1.8551135723,-1.2755929422 \backslash \mathrm{C}$ $,-0.9703132975,2.1946141527,1.2767526375 \backslash C,-0.9703132975,2.1946141527$, $-1.2767526375 \backslash \mathrm{H},-0.2017413192,2.9722566808,1.2918741221 \backslash \mathrm{H},-0.201741319$ $2,2.9722566808,-1.2918741221 \backslash \mathrm{H},-1.9538647806,2.6720946961,1.265561156 \backslash$ $\mathrm{H},-1.9538647806,2.6720946961,-1.265561156 \backslash \mathrm{H},-0.8602234089,1.5898369583$ , $2.1807502574 \backslash \mathrm{H},-0.8602234089,1.5898369583,-2.1807502574 \backslash \mathrm{H},-1.46290498$ $44,-1.3377983359,2.1799204496 \backslash \mathrm{H},-1.4629049844,-1.3377983359,-2.1799204$ $496 \backslash \mathrm{H},-2.8856170421,-1.9196256879,1.261787373 \backslash \mathrm{H},-2.8856170421,-1.91962$ $56879,-1.261787373 \backslash \mathrm{H},-1.3837390947,-2.8687723909,1.2946751552 \backslash \mathrm{H},-1.383$ $7390947,-2.8687723909,-1.2946751552 \backslash \backslash$ Version=IA32L-G03RevC.02\State=1$A^{\prime} \backslash H F=-671.8056477 \backslash S 2=0 . \backslash S 2-1=0 . \backslash S 2 A=0 . \backslash R M S D=7.034 e-09 \backslash R M S F=1.855 e-05 \backslash$ Dipole $=-0.679181,-0.4214241,0 . \backslash P G=C S \quad[S G(C 8 H 4 N 1 O 2), X(C 4 H 12)] \backslash \backslash @$

\section{5-hydroxy-TMIO-}

$1 \backslash 1 \backslash G I N C-L C 135 \backslash F O p t \backslash U B 3 L Y P \backslash 6-31 G(d) \backslash C 12 H 16 N 1 O 2(1-) \backslash J O H 501 \backslash 01-M a r-2006 \backslash$

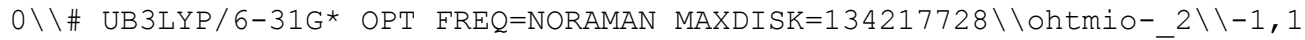
$\backslash \mathrm{N}, 1.7506388781,0.2523826329,-0.49035214 \backslash \mathrm{C}, 1.2669398888,-1.095 \overline{3} 622293$, $-0.0347190633 \backslash \mathrm{C},-0.2285608189,-0.8789719886,-0.0572943432 \backslash \mathrm{C},-0.5126659$ $952,0.4953878271,-0.0596826361 \backslash C,-1.8314394302,0.9455050085,-0.0284573$ $785 \backslash \mathrm{C},-2.8742855499,0.0093691522,0.0028704541 \backslash \mathrm{C},-1.2722540035,-1.80096$ $49895,-0.0253600497 \backslash \mathrm{C},-2.6034849713,-1.3591026798,0.0051559827 \backslash \mathrm{C}, 0.770$ $0531368,1.2929860737,-0.0347594125 \backslash \mathrm{H},-2.0569840249,2.0131513868,-0.025$ $0974729 \backslash 0,-4.2027870576,0.4051999313,0.0361475303 \backslash \mathrm{H},-1.0641081834,-2.8$ $693461583,-0.0195927574 \backslash \mathrm{H},-3.433950549,-2.0598667421,0.033662093 \backslash 0,3.0$ $589736493,0.5282143621,-0.1665803523 \backslash C, 1.1155742054,1.8542036503,1.368$ $629527 \backslash C, 0.840817099,2.4338759967,-1.0642094271 \backslash C, 1.7845498109,-2.1107$ $967649,-1.0671758873 \backslash \mathrm{C}, 1.8158419238,-1.4692041969,1.3647619174 \backslash \mathrm{H}, 0.194$ $3317661,3.2852081114,-0.8044098574 \backslash \mathrm{H}, 1.8837448606,2.7681710083,-1.0985$ $94356 \backslash \mathrm{H}, 0.569160585,2.0625872357,-2.0584612612 \backslash \mathrm{H}, 0.7488275281,2.877699$ $3612,1.5255244597 \backslash \mathrm{H}, 0.723714914,1.2236622716,2.1743921622 \backslash \mathrm{H}, 2.21533080$ $92,1.8278670173,1.3936199675 \backslash \mathrm{H}, 1.5264282898,-3.1484173033,-0.809624721$ $1 \backslash \mathrm{H}, 1.3867705114,-1.8751876434,-2.0603796673 \backslash \mathrm{H}, 2.8748678943,-2.0068318$ $609,-1.1029784951 \backslash \mathrm{H}, 1.8893804777,-2.5539058792,1.5212104079 \backslash \mathrm{H}, 2.813694$ $0446,-1.0054106091,1.3870354329 \backslash \mathrm{H}, 1.2073052675,-1.0494055051,2.1734442$ $379 \backslash \mathrm{H},-4.2089908457,1.3744833753,0.0276192831 \backslash \backslash$ Version=IA32L-G03RevC. 0 $2 \backslash \mathrm{State}=1-\mathrm{A} \backslash \mathrm{HF}=-672.0448344 \backslash \mathrm{S} 2=0 . \backslash \mathrm{S} 2-1=0 . \backslash \mathrm{S} 2 \mathrm{~A}=0 . \backslash \mathrm{RMSD}=8.417 \mathrm{e}-09 \backslash \mathrm{RMSF}=9$ $.770 e-06 \backslash$ Dipole $=-2.8532093,-0.0151798,0.2486366 \backslash \mathrm{PG}=\mathrm{C} 01 \quad[\mathrm{X}(\mathrm{C} 12 \mathrm{H} 16 \mathrm{~N} 102)]$ $11 @$

\section{1,1,3,3-tetraethylisoindoline-2-yloxyl• (28)}

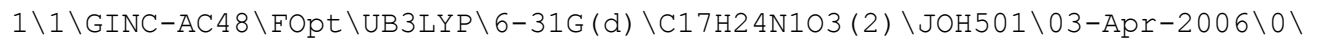

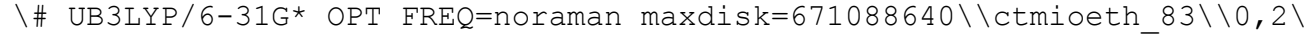
$0,3.6037739866,0.2639574447,-0.1685795621 \backslash N, 2.3375892691,0.29 \overline{3} 1813687$, $-0.0103873277 \backslash C, 1.5537148529,1.5740264057,-0.0019980241 \backslash C, 1.5347724444$ $,-0.9645355607,-0.0305665525 \backslash C, 0.1429580213,1.0134338816,0.0139638664 \backslash$ $C, 0.1366825858,-0.3899018985,-0.0107978744 \backslash C,-1.0618541924,1.716054122$ $6,0.0813805962 \backslash \mathrm{C},-1.0623929245,-1.0910892282,0.0047881754 \backslash \mathrm{H},-1.0720169$ $159,2.8000349365,0.1333713158 \backslash \mathrm{H},-1.092769289,-2.176204628,-0.013664966$ $6 \backslash C,-2.2681645514,1.0162980576,0.0942341663 \backslash C,-2.2734361645,-0.3855593$ $259,0.0513682285 \backslash \mathrm{H},-3.2090101963,1.5529158512,0.1419774881 \backslash \mathrm{C},-3.532762$ $8207,-1.1742422542,0.064935109 \backslash 0,-3.594330807,-2.3876273592,0.03569692$ $3 \backslash 0,-4.6475030077,-0.3993412557,0.1147454395 \backslash \mathrm{H},-5.3984535852,-1.021230$ 
$1964,0.1200542919 \backslash \mathrm{C}, 1.8722152345,-1.8362615681,1.2100393738 \backslash \mathrm{C}, 1.858773$ $0843,-1.8005899414,-1.2977135796 \backslash C, 1.8379459797,2.3871013879,1.2914454$ $572 \backslash C, 1.9121635151,2.3578835134,-1.2947356783 \backslash C, 3.2695470512,2.9087499$ $536,1.4620793219 \backslash \mathrm{H}, 3.0054647616,2.4082220668,-1.3333985942 \backslash \mathrm{H}, 1.5750297$ $532,1.7550188398,2.1467402761 \backslash \mathrm{H}, 1.6008270396,1.7505587196,-2.151117716$ $\backslash \mathrm{H}, 1.1328967943,3.2280837537,1.3128218824 \backslash \mathrm{C}, 1.3182911677,3.763052793,-$ $1.4383615439 \backslash \mathrm{H}, 2.924054366,-2.1301582607,1.1127722227 \backslash \mathrm{H}, 2.9073440586,-$ $2.1076849913,-1.2166654461 \backslash \mathrm{H}, 1.2787120429,-2.7564891366,1.1344686906 \backslash \mathrm{H}$ $, 1.254541817,-2.7152523393,-1.2420222481 \backslash C, 1.6512656046,-1.1931821574$, $2.5801967936 \backslash \mathrm{C}, 1.6368791023,-1.1185911994,-2.6485242271 \backslash \mathrm{H}, 1.8299321835$ $,-1.8279651029,-3.460947025 \backslash \mathrm{H}, 0.6094138214,-0.7556074943,-2.7628476271$ $\backslash \mathrm{H}, 2.3206796535,-0.2747773148,-2.7803172859 \backslash \mathrm{H}, 1.886380223,-1.911693528$ $9,3.3731805331 \backslash \mathrm{H}, 2.3027579325,-0.324907046,2.720325086 \backslash \mathrm{H}, 0.6135377191$, $-0.8715358084,2.7208495992 \backslash \mathrm{H}, 3.3652720602,3.4165455335,2.428676864 \backslash \mathrm{H}, 3$ $.9944055872,2.0924913499,1.4229105132 \backslash \mathrm{H}, 3.5398097988,3.6296722892,0.68$ $2979132 \backslash \mathrm{H}, 1.6649863474,4.2124548314,-2.3757116504 \backslash \mathrm{H}, 0.2238183741,3.747$ $8859557,-1.4747535728 \backslash \mathrm{H}, 1.6223882213,4.4305145401,-0.6254008442 \backslash \backslash$ Versi on $=I A 64 L-G 03 R e v D .01 \backslash$ State $=2-A \backslash H F=-942.6580319 \backslash S 2=0.754047 \backslash S 2-1=0 . \backslash S 2 A=$ $0.750012 \backslash \mathrm{RMSD}=8.117 \mathrm{e}-09 \backslash \mathrm{RMSF}=3.296 \mathrm{e}-06 \backslash \mathrm{Thermal}=0 . \backslash \mathrm{Dipole}=-0.7399572,0$. $6947129,0.0668274 \backslash P G=C 01 \quad[X(\mathrm{C} 17 \mathrm{H} 24 \mathrm{~N} 103)] \backslash \backslash \Theta$

\section{1,1,3,3-tetraethylisoindoline-2-yloxyl+}

$1 \backslash 1 \backslash G I N C-L C 120 \backslash F O p t \backslash U B 3 L Y P \backslash 6-31 G(d) \backslash C 17 H 24 N 103(1+) \backslash J O H 501 \backslash 04-A p r-2006 \backslash$

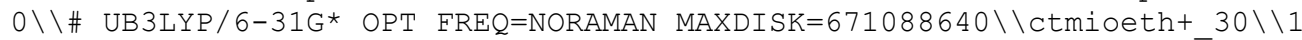
$, 1 \backslash 0,3.056192016,0.2308883786,-0.0087448423 \backslash \mathrm{N}, 1.8580737187,0.230 \overline{6} 25323$ $1,-0.01473102 \backslash \mathrm{C}, 1.0477975038,1.5007027761,-0.1134068924 \backslash \mathrm{C}, 1.0447550496$ $,-1.0366122378,0.0730591856 \backslash \mathrm{C},-0.3496014005,0.9282419126,-0.1238930007$ $\backslash C,-0.3512661231,-0.4596301995,0.0595804145 \backslash C,-1.5467543651,1.63294231$ $06,-0.2296542283 \backslash \mathrm{C},-1.5466319203,-1.1618017855,0.1400321964 \backslash \mathrm{H},-1.55531$ $7197,2.7101476298,-0.3692650362 \backslash \mathrm{H},-1.5865394516,-2.237953016,0.2789163$ $233 \backslash \mathrm{C},-2.7512904504,0.9314132675,-0.1524350528 \backslash \mathrm{C},-2.7527811052,-0.4576$ $382562,0.0357989655 \backslash \mathrm{H},-3.6942382443,1.4594456204,-0.2351694025 \backslash \mathrm{C},-4.01$ $65123352,-1.2549163201,0.1292747444 \backslash 0,-4.0400801739,-2.4556323156,0.29$ $11926707 \backslash 0,-5.1201751313,-0.4906778668,0.0128823087 \backslash \mathrm{H},-5.8902928943,-1$ $.0865707915,0.0841969111 \backslash \mathrm{C}, 1.4673456233,-1.8565929943,1.3206570993 \backslash \mathrm{C}, 1$ $.3080927287,-1.84668602,-1.259214744 \backslash C, 1.2921439629,2.3062511262,1.225$ $1020485 \backslash \mathrm{C}, 1.4927733317,2.318840611,-1.3539608668 \backslash \mathrm{C}, 2.6957744993,2.8427$ $401536,1.4956360669 \backslash \mathrm{H}, 2.5516698674,2.5670413864,-1.2371600977 \backslash \mathrm{H}, 0.9483$ $606092,1.6861915503,2.0585913068 \backslash C, 1.2481135814,1.6664570454,-2.716678$ $9695 \backslash \mathrm{H}, 0.5767355388,3.1338285476,1.1478697435 \backslash \mathrm{H}, 0.9402916162,3.2631475$ $378,-1.2850091381 \backslash \mathrm{H}, 2.5276532727,-2.1064019154,1.2215244725 \backslash \mathrm{C}, 2.712309$ $089,-2.3928691257,-1.5043646027 \backslash \mathrm{H}, 0.913999666,-2.7995519796,1.23954584$ $67 \backslash \mathrm{H}, 0.5850699284,-2.6684764829,-1.1913071061 \backslash \mathrm{C}, 1.1991175942,-1.209376$ $9054,2.6811937504 \backslash \mathrm{H}, 0.98269467,-1.2271637285,-2.1005432488 \backslash \mathrm{H}, 2.7117535$ $029,-2.9210174088,-2.4640523606 \backslash \mathrm{H}, 3.4687637236,-1.6039468092,-1.574620$ $5083 \backslash \mathrm{H}, 3.0289339315,-3.105317549,-0.7381776948 \backslash \mathrm{H}, 1.4384121288,-1.92600$ $84668,3.4724894029 \backslash \mathrm{H}, 1.8273737635,-0.327782881,2.8502106001 \backslash \mathrm{H}, 0.150338$ $9757,-0.9215406228,2.8007202161 \backslash \mathrm{H}, 2.6808705406,3.3742165481,2.45336449$ $46 \backslash \mathrm{H}, 3.4447666458,2.0486514322,1.5833006629 \backslash \mathrm{H}, 3.0318718612,3.550014118$ $2,0.7330788398 \backslash \mathrm{H}, 1.5030304777,2.3781196475,-3.507528939 \backslash \mathrm{H}, 1.8780238795$ $, 0.7830395867,-2.8682394952 \backslash \mathrm{H}, 0.2014518883,1.378093065,-2.8526164357 \backslash \backslash$ Version=IA32L-G03RevC.02\State $=1-A \backslash H F=-942.4109637 \backslash S 2=0 . \backslash S 2-1=0 . \backslash S 2 A=0$ $. \backslash \mathrm{RMSD}=5.320 \mathrm{e}-09 \backslash \mathrm{RMSF}=1.085 \mathrm{e}-05 \backslash \mathrm{Dipole}=1.3650771,0.9907315,-0.1209074 \backslash$ $\mathrm{PG}=\mathrm{C} 01 \quad[\mathrm{X}(\mathrm{C} 17 \mathrm{H} 24 \mathrm{~N} 1 \mathrm{O} 3)] \backslash \backslash \mathrm{Q}$

\section{1,1,3,3-tetraethylisoindoline-2-yloxyl-}

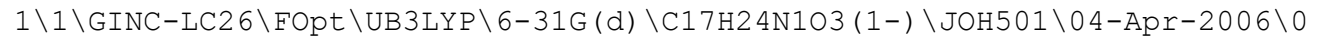
$\backslash \backslash \#$ UB3LYP/6-31G* OPT FREQ=NORAMAN MAXDISK=671088640 \Ctmioeth- $21 \backslash \backslash-1$ $, 1 \backslash 0,3.1851004306,0.2521959774,-0.0611919442 \backslash \mathrm{N}, 1.8493946271,0.2 \overline{6} 607180$ $92,-0.3554110307 \backslash \mathrm{C}, 1.1287915315,1.5145859259,-0.0220947014 \backslash \mathrm{C}, 1.0667612$ $974,-0.9551242895,-0.0001595078 \backslash C,-0.2964296766,1.0045824698,-0.068538$ $1582 \backslash C,-0.3384865345,-0.3999999775,-0.0338986558 \backslash C,-1.4901945883,1.739$ $9523606,-0.0301193347 \backslash \mathrm{C},-1.5519310568,-1.069493984,-0.0042777838 \backslash \mathrm{H},-1$. 
$4653889448,2.8279113053,-0.0350648056 \backslash \mathrm{H},-1.6077014536,-2.1533284758,0$. $0047712862 \backslash \mathrm{C},-2.7123537828,1.0762536795,0.0095045563 \backslash \mathrm{C},-2.7614283545,-$ $0.3319070891,0.0219067474 \backslash \mathrm{H},-3.6401247462,1.6392588749,0.0316056583 \backslash \mathrm{C}$, $-4.0278677346,-1.0635588004,0.0634934147 \backslash 0,-4.1687202123,-2.2804969014$ $, 0.0851225992 \backslash 0,-5.1428443294,-0.2525545532,0.0741077425 \backslash \mathrm{H},-5.88240786$ $36,-0.8853388943,0.0980300644 \backslash C, 1.4694729972,-1.4781204411,1.409578933$ $6 \backslash C, 1.2779349212,-2.016371484,-1.1138882223 \backslash C, 1.4112523792,2.126026698$ $5,1.4012426094 \backslash C, 1.4825858463,2.5747860373,-1.1021810828 \backslash C, 2.803320179$ $2,2.7285784478,1.6374004171 \backslash \mathrm{H}, 2.5738076279,2.667054272,-1.0782001431 \backslash \mathrm{H}$ $, 1.2403528025,1.3362525271,2.1417908992 \backslash C, 1.0482503432,2.2112948811,-2$ $.5235819717 \backslash \mathrm{H}, 0.640828503,2.8906231838,1.591960055 \backslash \mathrm{H}, 1.0643621775,3.55$ $20829381,-0.814578947 \backslash \mathrm{H}, 2.5616333384,-1.3717776349,1.4346258803 \backslash \mathrm{C}, 2.73$ $02769249,-2.4829314408,-1.2889389856 \backslash C, 1.0321673553,-2.893976783,1.803$ $0623075 \backslash \mathrm{H}, 0.6178981078,-2.8795096782,-0.9372042566 \backslash \mathrm{H}, 1.076361208,-0.77$ $68028114,2.1559917961 \backslash \mathrm{H}, 0.9403075094,-1.550694179,-2.0480937445 \backslash \mathrm{H}, 2.84$ $02400555,-3.0669406497,-2.2138306506 \backslash \mathrm{H}, 3.3861337327,-1.6075370595,-1.3$ $038231567 \backslash \mathrm{H}, 3.0561385357,-3.1206041104,-0.4570691899 \backslash \mathrm{H}, 1.3760866854,-3$ $.1315136752,2.8199340411 \backslash \mathrm{H},-0.0593358167,-3.0058783795,1.7954642985 \backslash \mathrm{H}$, $1.4468876466,-3.6575302902,1.1352217493 \backslash \mathrm{H}, 2.9127415846,3.0463085323,2$. $6852859145 \backslash \mathrm{H}, 3.5504753028,1.972700783,1.3886691755 \backslash \mathrm{H}, 2.9793563275,3.61$ $13687078,1.0099012683 \backslash \mathrm{H}, 1.4271844162,2.9428865325,-3.2501691938 \backslash \mathrm{H}, 1.44$ $11167795,1.2236199417,-2.7816323628 \backslash \mathrm{H},-0.0437353007,2.1742721263,-2.62$ $10790935 \backslash \backslash$ Version=IA32L-G03RevC.02 \State=1-A \HF=-942.6621638\S2=0. $\backslash \mathrm{S} 2-$ $1=0 . \backslash \mathrm{S} 2 \mathrm{~A}=0 . \backslash \mathrm{RMSD}=4.976 \mathrm{e}-09 \backslash \mathrm{RMSF}=3.291 \mathrm{e}-06 \backslash \mathrm{Dipole}=-1.8733083,0.469541,0$ $.2138175 \backslash \mathrm{PG}=\mathrm{C} 01 \quad[\mathrm{X}(\mathrm{C} 17 \mathrm{H} 24 \mathrm{~N} 1 \mathrm{O} 3)] \backslash \backslash \mathrm{Q}$

\section{4-carboxy-TMIO•(29)}

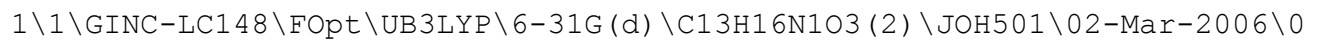

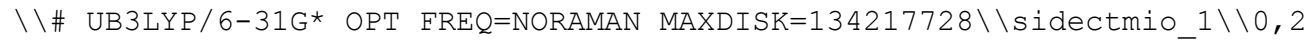
$\backslash 0,-3.1623362634,0.6606757573,-0.0975193111 \backslash \mathrm{N},-1.8898202736,0.6 \overline{2} 488956$ $63,-0.0609776274 \backslash C,-1.0743457199,1.8590648082,-0.0492192825 \backslash C,-1.13419$ $52656,-0.667783494,-0.0261214608 \backslash C, 0.3133109534,1.2612564044,-0.003525$ $0496 \backslash \mathrm{C}, 0.3012253503,-0.143650755,0.0098716722 \backslash \mathrm{C}, 1.5015104946,1.9867999$ $149,0.0235206772 \backslash \mathrm{C}, 1.5356788087,-0.8311090645,0.0521301884 \backslash \mathrm{H}, 1.4796334$ $242,3.0736069386,0.012249306 \backslash C, 1.6427000255,-2.3173978443,0.0699891801$ $\backslash C, 2.7171307256,1.3066922109,0.0651783085 \backslash C, 2.7287609006,-0.0839262903$ $, 0.0791846033 \backslash \mathrm{H}, 3.65366459,1.8561967676,0.0867490367 \backslash \mathrm{C},-1.4934832474,-$ $1.453437304,-1.3018127662 \backslash \mathrm{C},-1.5664263168,-1.4276304454,1.242596538 \backslash \mathrm{C}$, $-1.3483429171,2.6687439804,-1.3309371926 \backslash C,-1.4211626205,2.6928886112$, $1.1989368038 \backslash \mathrm{H},-2.4166103597,2.8976645745,-1.3885392883 \backslash \mathrm{H},-2.490869456$ $5,2.9221724374,1.1906854483 \backslash \mathrm{H},-1.0650175347,2.0995804929,-2.2217197971$ $\backslash \mathrm{H},-1.1897115609,2.1410642628,2.1152528204 \backslash \mathrm{H},-0.7855407021,3.608558616$ $7,-1.3249917643 \backslash \mathrm{H},-0.8585140093,3.6327740823,1.2073422994 \backslash \mathrm{H},-2.5828154$ $51,-1.5410575091,-1.3556146847 \backslash \mathrm{H},-2.657062819,-1.5152551113,1.23551594$ $42 \backslash \mathrm{H},-1.0466817029,-2.4472754506,-1.2804677995 \backslash \mathrm{H},-1.1192960871,-2.4212$ $467288,1.2671683918 \backslash \mathrm{H},-1.1468296489,-0.922167235,-2.194882568 \backslash \mathrm{H},-1.271$ $5733422,-0.8779168463,2.1430687197 \backslash 0,0.7263374597,-3.1172337615,0.0515$ $635897 \backslash 0,2.9316359001,-2.7495035536,0.1115810132 \backslash \mathrm{H}, 2.8736282017,-3.722$ $6756076,0.1196665252 \backslash \mathrm{H}, 3.6730745741,-0.6128225803,0.1116051487 \backslash \backslash$ Versio $\mathrm{n}=\mathrm{IA} 32 \mathrm{~L}-\mathrm{G} 03 \mathrm{RevC} .02 \backslash \mathrm{State}=2-\mathrm{A} \backslash \mathrm{HF}=-785.4061726 \backslash \mathrm{S} 2=0.753797 \backslash \mathrm{S} 2-1=0 . \backslash \mathrm{S} 2 \mathrm{~A}=0$ $.750011 \backslash \mathrm{RMSD}=3.561 \mathrm{e}-09 \backslash \mathrm{RMSF}=8.477 \mathrm{e}-06 \backslash \mathrm{Dipole}=1.5952857,0.4515618,0.041$ $7375 \backslash P G=C 01 \quad[X(C 13 \mathrm{H} 16 \mathrm{~N} 103)] \backslash \backslash @$

\section{4-carboxy-TMIO+}

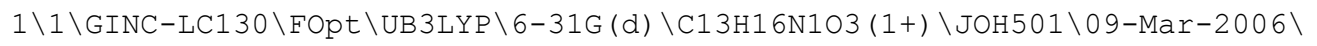
$0 \backslash \backslash \#$ UB3LYP/6-31G* OPT FREQ=NORAMAN MAXDISK=268435456\\sidectmio+ $1 \backslash \backslash 1$ $, 1 \backslash 0,-2.6308262601,-1.7389236088,0.0097729998 \backslash \mathrm{N},-1.7723082297,-0 . \overline{9} 1306$ $19924,0.0114049493 \backslash C,-2.0716104134,0.5662296484,0.0438767259 \backslash C,-0.2916$ $452677,-1.2652647131,-0.0189628049 \backslash C,-0.668811616,1.1211101604,0.03173$ $70301 \backslash C, 0.3205075058,0.1298993754,-0.0027215832 \backslash C,-0.354680218,2.47929$ $39221,0.0521015196 \backslash \mathrm{C}, 1.6779859605,0.5167010978,-0.017320145 \backslash \mathrm{H},-1.13852$ $23509,3.2310528181,0.0787633751 \backslash \mathrm{C}, 2.7982222412,-0.4734477009,-0.053874$ $4751 \backslash C, 0.984189144,2.8589303991,0.0376854456 \backslash C, 1.9829047671,1.88696321$ 
$2,0.0034297205 \backslash \mathrm{H}, 1.2533855942,3.9099271867,0.053035493 \backslash \mathrm{C},-0.0862208677$ $,-2.0770661614,-1.3223610454 \backslash C,-0.0449881896,-2.1249558169,1.246042515$ $8 \backslash \mathrm{C},-2.9084999251,0.8944411504,-1.2140778037 \backslash \mathrm{C},-2.8675207504,0.8466944$ $545,1.3394097061 \backslash \mathrm{H},-3.8579450057,0.352689148,-1.196771027 \backslash \mathrm{H},-3.8174734$ $241,0.3056117391,1.3322495109 \backslash \mathrm{H},-2.3635162325,0.6582835287,-2.13179298$ $47 \backslash \mathrm{H},-2.2936312933,0.5764684049,2.2297033992 \backslash \mathrm{H},-3.1165650064,1.9678607$ $714,-1.2060276477 \backslash \mathrm{H},-3.0747839264,1.9195917063,1.3783228846 \backslash \mathrm{H},-0.71275$ $58429,-2.9727824179,-1.3077009713 \backslash \mathrm{H},-0.6721471957,-3.0199216627,1.2180$ $807833 \backslash \mathrm{H}, 0.9637312301,-2.3675296931,-1.355926616 \backslash \mathrm{H}, 1.0053434707,-2.415$ $7737943,1.2350662594 \backslash \mathrm{H},-0.321436447,-1.4764538723,-2.2056654163 \backslash \mathrm{H},-0.2$ $51478048,-1.5578467472,2.1582001404 \backslash 0,2.6617338579,-1.6834769481,-0.07$ $42619972 \backslash 0,4.0050814343,0.1142126385,-0.0623865735 \backslash \mathrm{H}, 4.6733325269,-0.5$ $976172695,-0.0864515748 \backslash \mathrm{H}, 3.023713077,2.1862032817,-0.0077045224 \backslash \backslash$ Vers ion $=I A 32 L-G 03 R e v C .02 \backslash$ State $=1-A \backslash H F=-785.1578603 \backslash S 2=0 . \backslash S 2-1=0 . \backslash S 2 A=0 . \backslash R M$ $\mathrm{SD}=4.913 \mathrm{e}-09 \backslash \mathrm{RMSF}=3.856 \mathrm{e}-06 \backslash \mathrm{Dipole}=-1.2728805,0.5638087,0.0310071 \backslash \mathrm{PG}=\mathrm{C}$ $01[\mathrm{X}(\mathrm{C} 13 \mathrm{H} 16 \mathrm{~N} 103)] \backslash \backslash @$

\section{4-carboxy-TMIO-}

$1 \backslash 1 \backslash G I N C-L C 28 \backslash F O p t \backslash U B 3 L Y P \backslash 6-31 G(d) \backslash C 13 H 16 N 103(1-) \backslash J O H 501 \backslash 06-M a r-2006 \backslash 0$

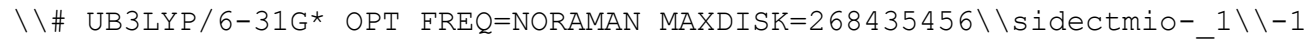
$, 1 \backslash 0,-3.1873853958,0.594228879,-0.0423010508 \backslash N,-1.876675504,0.55098379$ $49,-0.3197332653 \backslash C,-1.1172233663,1.7989219245,-0.1671990491 \backslash C,-1.11934$ $09725,-0.657239475,0.1022608597 \backslash C, 0.2844662628,1.2567054423,-0.2479226$ $142 \backslash \mathrm{C}, 0.3011972744,-0.1358452184,-0.0765134536 \backslash \mathrm{C}, 1.4522541012,2.003847$ $435,-0.3863814913 \backslash \mathrm{C}, 1.5537570118,-0.8097543176,-0.0211617224 \backslash \mathrm{H}, 1.40454$ $30333,3.0838911894,-0.5040627488 \backslash C, 1.6965701119,-2.245244017,0.2245799$ $309 \backslash \mathrm{C}, 2.6902898789,1.3367667013,-0.36358673 \backslash \mathrm{C}, 2.7387656094,-0.03382863$ $64,-0.1887208557 \backslash \mathrm{H}, 3.6166995244,1.8989706826,-0.4711496669 \backslash \mathrm{C},-1.547979$ $7052,-1.78062238,-0.8656753164 \backslash \mathrm{C},-1.4469836435,-1.0775415135,1.5579790$ $013 \backslash \mathrm{C},-1.5117172281,2.7324104629,-1.3302632979 \backslash \mathrm{C},-1.4222795699,2.51585$ $42698,1.1772811909 \backslash \mathrm{H},-2.6004999263,2.8489195742,-1.3034817502 \backslash \mathrm{H},-2.508$ $410811,2.4541857075,1.3119060028 \backslash \mathrm{H},-1.2390803816,2.2772740706,-2.28855$ $84961 \backslash \mathrm{H},-0.9374946615,2.0087436249,2.0183319738 \backslash \mathrm{H},-1.0400162801,3.7214$ $964366,-1.254226614 \backslash \mathrm{H},-1.1054925329,3.5668211138,1.1799716 \backslash \mathrm{H},-2.642809$ $1647,-1.8151284946,-0.8457272312 \backslash \mathrm{H},-2.5367702233,-1.0539518715,1.65899$ $73802 \backslash \mathrm{H},-1.1329666206,-2.7511550423,-0.5858816482 \backslash \mathrm{H},-1.0595876315,-2.0$ $746201352,1.7825357054 \backslash \mathrm{H},-1.2380479439,-1.5295804516,-1.8870190023 \backslash \mathrm{H},-$ $1.0220814553,-0.3674022389,2.2777396614 \backslash 0,0.8503990219,-3.0617640555,0$ $.5778176396 \backslash 0,2.9936009654,-2.7031801137,0.0337662358 \backslash \mathrm{H}, 2.9181894491$, $3.6468230228,0.2601668085 \backslash \mathrm{H}, 3.6969828329,-0.539389453,-0.1537304276 \backslash \backslash \mathrm{V}$ ersion=IA32L-G03RevC.02 \State $=1-A \backslash H F=-785.4048972 \backslash \mathrm{S} 2=0 . \backslash \mathrm{S} 2-1=0 . \backslash \mathrm{S} 2 \mathrm{~A}=0$. $\backslash \mathrm{RMSD}=2.459 \mathrm{e}-09 \backslash \mathrm{RMSF}=8.390 \mathrm{e}-06 \backslash \mathrm{Dipole}=2.330781,0.1175545,0.0355819 \backslash \mathrm{PG}=$ $\mathrm{C} 01[\mathrm{X}(\mathrm{C} 13 \mathrm{H} 16 \mathrm{~N} 103)] \backslash \backslash \mathrm{e}$

\section{4,7-dicarboxy-TMIO•(30)}

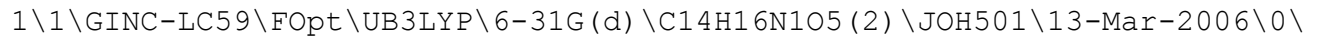

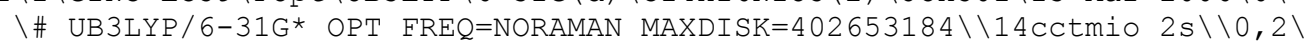
$\mathrm{N}, 0 ., 0 ., 0 . \backslash 0,1.2704007871,-0.079245675,0 . \backslash \mathrm{C},-4.6043755622,0.2 \overline{9} 62915761$ $, 0.69212 \backslash C,-4.6043755622,0.2962915761,-0.69212 \backslash 0,-4.7274280231,-0.0484$ $662763,3.34605 \backslash 0,-4.7274280231,-0.0484662763,-3.34605 \backslash \mathrm{H},-5.5438146873$, $0.3038934327,1.23034 \backslash \mathrm{H},-5.5438146873,0.3038934327,-1.23034 \backslash \mathrm{H},-4.691454$ $7183,0.0360781223,4.31713 \backslash \mathrm{H},-4.6914547183,0.0360781223,-4.31713 \backslash \mathrm{C},-3.4$ $958075685,0.3185781628,2.90602 \backslash \mathrm{C},-3.4958075685,0.3185781628,-2.90602 \backslash \mathrm{C}$ $,-3.4005898774,0.2898744679,1.41392 \backslash C,-3.4005898774,0.2898744679,-1.41$ $392 \backslash 0,-2.6238856112,0.6369420887,3.68922 \backslash 0,-2.6238856112,0.6369420887$, $-3.68922 \backslash C,-2.1803744526,0.2737857237,0.70683 \backslash C,-2.1803744526,0.273785$ $7237,-0.70683 \backslash \mathrm{C},-0.7599933922,0.2117159745,1.2619 \backslash \mathrm{C},-0.7599933922,0.21$ $17159745,-1.2619 \backslash C,-0.2763621954,1.5320876767,1.90072 \backslash C,-0.2763621954$, $1.5320876767,-1.90072 \backslash C,-0.4696883873,-0.9906801849,2.17925 \backslash C,-0.46968$ $83873,-0.9906801849,-2.17925 \backslash \mathrm{H}, 0.8095086982,1.4730495676,2.02118 \backslash \mathrm{H}, 0.8$ $095086982,1.4730495676,-2.02118 \backslash \mathrm{H},-0.7484196912,1.6852939871,2.87063 \backslash \mathrm{H}$ $,-0.7484196912,1.6852939871,-2.87063 \backslash \mathrm{H},-0.504493105,2.3858266814,-1.25$ $336 \backslash \mathrm{H},-0.504493105,2.3858266814,1.25336 \backslash \mathrm{H}, 0.6159246666,-1.1046087224,2$ 
$.24877 \backslash \mathrm{H}, 0.6159246666,-1.1046087224,-2.24877 \backslash \mathrm{H},-0.8788334042,-0.834254$ $4014,3.17717 \backslash \mathrm{H},-0.8788334042,-0.8342544014,-3.17717 \backslash \mathrm{H},-0.884431791,-1$. $9118362376,-1.75655 \backslash \mathrm{H},-0.884431791,-1.9118362376,1.75655 \backslash \backslash$ Version=IA32 $\mathrm{L}-\mathrm{G} 03 \mathrm{RevC} .02 \backslash \mathrm{State}=2-\mathrm{A} \cdot \backslash \mathrm{HF}=-973.9696301 \backslash \mathrm{S} 2=0.753764 \backslash \mathrm{S} 2-1=0 . \backslash \mathrm{S} 2 \mathrm{~A}=0.7500$ $1 \backslash \mathrm{RMSD}=1.744 \mathrm{e}-09 \backslash \mathrm{RMSF}=6.655 \mathrm{e}-05 \backslash \mathrm{Dipole}=2.016019,-0.1842596,0 . \backslash \mathrm{PG}=\mathrm{CS} \quad[\mathrm{S}$ $\mathrm{G}(\mathrm{N} 1 \mathrm{O} 1), \mathrm{X}(\mathrm{C} 14 \mathrm{H} 16 \mathrm{O} 4)] \backslash \backslash \mathrm{e}$

\section{4,7-dicarboxy-TMIO+}

$1 \backslash 1 \backslash G I N C-L C 144 \backslash$ FOpt \UB3LYP \6-31G (d) \C14H16N105 (1+) \JOH501 \18-Mar-2006\

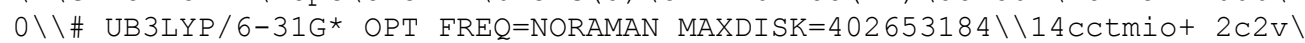
$\backslash 1,1 \backslash 0,0 ., 0 .,-3.3465759344 \backslash \mathrm{N}, 0 ., 0 .,-2.1543298384 \backslash \mathrm{C}, 1.2741120676, \overline{0},,-1$. $3475414981 \backslash C,-1.2741120676,0 .,-1.3475414981 \backslash C, 0.7038223458,0 ., 0.064848$ $9672 \backslash C,-0.7038223458,0 ., 0.0648489672 \backslash C, 1.4191983124,0 ., 1.283230105 \backslash C,-$ $1.4191983124,0 ., 1.283230105 \backslash C, 2.9179609495,0 ., 1.3620931702 \backslash C,-2.917960$ $9495,0 ., 1.3620931702 \backslash \mathrm{C}, 0.6942044499,0 ., 2.4799687986 \backslash \mathrm{C},-0.6942044499,0$. $, 2.4799687986 \backslash \mathrm{H}, 1.2304303813,0 ., 3.4203426596 \backslash \mathrm{H},-1.2304303813,0 ., 3.4203$ $426596 \backslash C,-2.0298443001,-1.286678546,-1.7713460627 \backslash C,-2.0298443001,1.28$ $6678546,-1.7713460627 \backslash \mathrm{C}, 2.0298443001,1.286678546,-1.7713460627 \backslash \mathrm{C}, 2.029$ $8443001,-1.286678546,-1.7713460627 \backslash \mathrm{H},-2.2194034485,-1.2637282292,-2.84$ $76011409 \backslash \mathrm{H},-2.2194034485,1.2637282292,-2.8476011409 \backslash \mathrm{H}, 2.2194034485,1.2$ $637282292,-2.8476011409 \backslash \mathrm{H}, 2.2194034485,-1.2637282292,-2.8476011409 \backslash \mathrm{H},-$ $2.9778499733,-1.3004852844,-1.2356860149 \backslash \mathrm{H},-2.9778499733,1.3004852844$, $-1.2356860149 \backslash \mathrm{H}, 2.9778499733,1.3004852844,-1.2356860149 \backslash \mathrm{H}, 2.9778499733$ $,-1.3004852844,-1.2356860149 \backslash \mathrm{H},-1.4571387422,-2.1834206095,-1.51813947$ $64 \backslash \mathrm{H},-1.4571387422,2.1834206095,-1.5181394764 \backslash \mathrm{H}, 1.4571387422,2.1834206$ $095,-1.5181394764 \backslash \mathrm{H}, 1.4571387422,-2.1834206095,-1.5181394764 \backslash 0,3.67442$ $9085,0 ., 0.4094382203 \backslash 0,-3.674429085,0 ., 0.4094382203 \backslash 0,3.3586938406,0 .$, $2.6296258014 \backslash 0,-3.3586938406,0 ., 2.6296258014 \backslash \mathrm{H}, 4.3349494219,0 ., 2.59701$ $2098 \backslash H,-4.3349494219,0 ., 2.597012098 \backslash \backslash$ Version=IA32L-G03RevC.02 State=1$\mathrm{A} 1 \backslash \mathrm{HF}=-973.7245342 \backslash \mathrm{S} 2=0 . \backslash \mathrm{S} 2-1=0 . \backslash \mathrm{S} 2 \mathrm{~A}=0 . \backslash \mathrm{RMSD}=9.209 \mathrm{e}-09 \backslash \mathrm{RMSF}=6.549 \mathrm{e}-05 \backslash$ Dipole $=0 ., 0 .,-0.2566693 \backslash \mathrm{PG}=\mathrm{C} 02 \mathrm{~V} \quad[\mathrm{C} 2(\mathrm{~N} 1 \mathrm{O} 1), \mathrm{SGV}(\mathrm{C} 10 \mathrm{H} 4 \mathrm{O} 4), \mathrm{X}(\mathrm{C} 4 \mathrm{H} 12)] \backslash \backslash \mathrm{e}$

\section{4,7-dicarboxy-TMIO-}

$1 \backslash 1 \backslash G I N C-L C 146 \backslash$ FOpt \UB3LYP \6-31G (d) \C14H16N1O5 (1-) \JOH501 \21-Mar-2006 $0 \backslash \backslash \#$ UB3LYP/6-31G* OPT FREQ=NORAMAN MAXDISK=402653184\\14cctmio-_4c2v $\backslash-1,1 \backslash 0,0 ., 0 ., 3.4255307575 \backslash \mathrm{N}, 0 ., 0 ., 2.1308743748 \backslash \mathrm{C}, 0 ., 1.259633700 \overline{3}, 1.37$ $73504398 \backslash \mathrm{C}, 0 \ldots,-1.2596337003,1.3773504398 \backslash \mathrm{C}, 0 ., 0.6999875323,-0.03495149$ $45 \backslash \mathrm{C}, 0 .,-0.6999875323,-0.0349514945 \backslash \mathrm{C}, 0 ., 1.4417850422,-1.25535038 \backslash \mathrm{C}, 0$. $,-1.4417850422,-1.25535038 \backslash \mathrm{C}, 0 ., 2.891734056,-1.3502171137 \backslash \mathrm{C}, 0 .,-2.8917$ $34056,-1.3502171137 \backslash \mathrm{C}, 0 ., 0.6838777237,-2.4685014795 \backslash \mathrm{C}, 0 .,-0.6838777237$ $,-2.4685014795 \backslash \mathrm{H}, 0 ., 1.2173420279,-3.4115164706 \backslash \mathrm{H}, 0 .,-1.2173420279,-3.4$ $115164706 \backslash \mathrm{C},-1.2675496184,-2.0656071539,1.7534223746 \backslash \mathrm{C}, 1.2675496184,-2$ $.0656071539,1.7534223746 \backslash C, 1.2675496184,2.0656071539,1.7534223746 \backslash \mathrm{C},-1$ $.2675496184,2.0656071539,1.7534223746 \backslash \mathrm{H},-1.2977699587,-2.1588671235,2$. $8441499458 \backslash \mathrm{H}, 1.2977699587,-2.1588671235,2.8441499458 \backslash \mathrm{H}, 1.2977699587,2$. $1588671235,2.8441499458 \backslash \mathrm{H},-1.2977699587,2.1588671235,2.8441499458 \backslash \mathrm{H},-1$ $.2534957193,-3.0539604084,1.2907492156 \backslash \mathrm{H}, 1.2534957193,-3.0539604084,1$. $2907492156 \backslash \mathrm{H}, 1.2534957193,3.0539604084,1.2907492156 \backslash \mathrm{H},-1.2534957193,3$. $0539604084,1.2907492156 \backslash \mathrm{H},-2.16564744,-1.5254332433,1.4311291642 \backslash \mathrm{H}, 2.1$ $6564744,-1.5254332433,1.4311291642 \backslash \mathrm{H}, 2.16564744,1.5254332433,1.4311291$ $642 \backslash \mathrm{H},-2.16564744,1.5254332433,1.4311291642 \backslash 0,0 ., 3.7463349186,-0.46015$ $27931 \backslash 0,0 .,-3.7463349186,-0.4601527931 \backslash 0,0 ., 3.3504250485,-2.6633604109$ $\backslash 0,0 .,-3.3504250485,-2.6633604109 \backslash \mathrm{H}, 0 ., 4.3160208841,-2.5436662185 \backslash \mathrm{H}, 0$. $,-4.3160208841,-2.5436662185 \backslash \backslash$ Version=IA32L-G03RevC.02 $\backslash$ State $=1-A 1 \backslash H F=-$ $973.986741 \backslash S 2=0 . \backslash S 2-1=0 . \backslash S 2 A=0 . \backslash R M S D=7.634 e-09 \backslash R M S F=3.914 e-05 \backslash \mathrm{Dipole}=0$ ., $0 .,-1.4887701 \backslash \mathrm{PG}=\mathrm{C} 02 \mathrm{~V} \quad[\mathrm{C} 2(\mathrm{~N} 1 \mathrm{O} 1), \mathrm{SGV}(\mathrm{C} 10 \mathrm{H} 4 \mathrm{O} 4), \mathrm{X}(\mathrm{C} 4 \mathrm{H} 12)] \backslash \backslash \mathrm{e}$

\section{4,6-dicarboxy-TMIO•(31)}

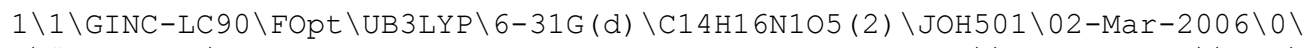

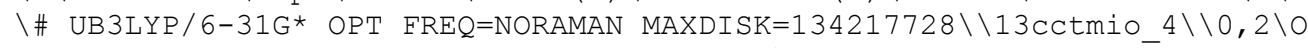
$,-3.8334066345,-0.334397062,-0.0121839578 \backslash N,-2.5609573881,-0 . \overline{2} 95337249$ 
$1,-0.0073480672 \backslash \mathrm{C},-1.8103238316,1.0006053171,-0.0179246053 \backslash \mathrm{C},-1.741689$ $7481,-1.5269659453,0.0090376883 \backslash C,-0.3729340047,0.4839106133,-0.006580$ $7763 \backslash \mathrm{C},-0.356082729,-0.9229721101,0.0083072143 \backslash \mathrm{C}, 0.859265165,1.1777799$ $199,-0.0088966545 \backslash C, 0.8313587103,-1.6409604831,0.0206748148 \backslash C, 0.961341$ $2442,2.6664689387,-0.0240853941 \backslash \mathrm{C}, 2.0553834837,0.4403545273,0.00368469$ $18 \backslash \mathrm{C}, 2.050232902,-0.9549790823,0.0183630134 \backslash \mathrm{H}, 2.9994834081,0.967522409$ $2,0.001926545 \backslash \mathrm{C},-2.0418243383,-2.3633358427,-1.2492310086 \backslash \mathrm{C},-2.0521351$ $38,-2.3365083502,1.2822816244 \backslash \mathrm{C},-2.2036293815,1.7588183207,-1.30082951$ $97 \backslash \mathrm{C},-2.2141702524,1.7856933252,1.2453800253 \backslash \mathrm{H},-3.2943847087,1.8392894$ $37,-1.3263905879 \backslash \mathrm{H},-3.3050980945,1.8665120842,1.2602279135 \backslash \mathrm{H},-1.878852$ $899,1.2105482566,-2.1917681185 \backslash \mathrm{H},-1.8967755745,1.2564187888,2.15035850$ $19 \backslash \mathrm{H},-1.762199632,2.7550885639,-1.3109325978 \backslash \mathrm{H},-1.7728160899,2.7820206$ $815,1.2380963728 \backslash \mathrm{H},-3.1086679709,-2.604282207,-1.2724417947 \backslash \mathrm{H},-3.11914$ $43034,-2.5770449644,1.3018936499 \backslash \mathrm{H},-1.4684426705,-3.2964394604,-1.2410$ $612961 \backslash \mathrm{H},-1.4787458258,-3.2695010061,1.2985768084 \backslash \mathrm{H},-1.7900138059,-1.8$ $084284703,-2.1583236363 \backslash \mathrm{H},-1.807720601,-1.7624083404,2.1814180056 \backslash \mathrm{C}, 3$. $3036119598,-1.7560889905,0.0318648898 \backslash 0,0.0399539424,3.4595145602,-0.0$ $361528624 \backslash 0,2.2484158347,3.0999061797,-0.023388026 \backslash \mathrm{H}, 2.1932907227,4.07$ $33311722,-0.0338231529 \backslash 0,3.3455662396,-2.9698927987,0.044871518 \backslash 0,4.42$ $36667591,-0.9917643983,0.0282733652 \backslash \mathrm{H}, 5.1722403258,-1.616669648,0.0378$ $771667 \backslash \mathrm{H}, 0.8405560586,-2.7264493481,0.0321663742 \backslash \backslash$ Version=IA32L-G03Rev C. $02 \backslash$ State $=2-A \backslash H F=-973.9781336 \backslash \mathrm{S} 2=0.753799 \backslash \mathrm{S} 2-1=0 . \backslash \mathrm{S} 2 \mathrm{~A}=0.750011 \backslash \mathrm{RMSD}=6$ $.260 e-09 \backslash \mathrm{RMSF}=1.437 \mathrm{e}-06 \backslash \mathrm{Dipole}=1.3871458,0.2844673,0.0026622 \backslash \mathrm{PG}=\mathrm{C} 01 \quad[\mathrm{X}$ (C14H16N105) ] \\@

\section{4,6-dicarboxy-TMIO+}

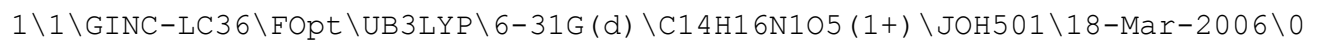

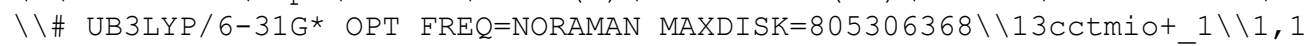
$\backslash 0,-3.6475803607,-0.9503110154,-0.0000784601 \backslash \mathrm{N},-2.4798875614,-0.715775$ $2691,0.0000784849 \backslash \mathrm{C},-1.9274154217,0.7041218075,-0.0000177008 \backslash \mathrm{C},-1.4344$ $840702,-1.8039496823,0.000038323 \backslash \mathrm{C},-0.4316830988,0.4144945576,0.000022$ $5967 \backslash \mathrm{C},-0.1806298883,-0.9652576277,0.0000498807 \backslash \mathrm{C}, 0.6591535833,1.30984$ $17479,0.0000133827 \backslash \mathrm{C}, 1.1102984123,-1.478948077,0.0000693916 \backslash \mathrm{C}, 0.484006$ $8108,2.7962452432,-0.0000250052 \backslash C, 1.9598007024,0.7829604941,0.00003904$ $65 \backslash \mathrm{C}, 2.1896125447,-0.5947189517,0.0000681724 \backslash \mathrm{H}, 2.8018845221,1.46260481$ $87,0.000032509 \backslash \mathrm{C},-1.6458857179,-2.6495555436,-1.2771909136 \backslash \mathrm{C},-1.645870$ $5813,-2.6496090268,1.2772356252 \backslash \mathrm{C},-2.4855905295,1.3656341249,-1.284920$ $4151 \backslash \mathrm{C},-2.4856285488,1.3658127751,1.28477531 \backslash \mathrm{H},-3.5784753214,1.3569616$ $802,-1.2627661246 \backslash \mathrm{H},-3.5785126086,1.3571467336,1.2625857087 \backslash \mathrm{H},-2.13095$ $01444,0.8524594272,-2.1833803942 \backslash \mathrm{H},-2.1310218719,0.8527580671,2.183317$ $0559 \backslash \mathrm{H},-2.1296403396,2.3956703263,-1.2959086235 \backslash \mathrm{H},-2.1296688396,2.3958$ $468911,1.2956331065 \backslash \mathrm{H},-2.6281992316,-3.1290584269,-1.2619601559 \backslash \mathrm{H},-2.6$ $281809834,-3.1291178848,1.2619936765 \backslash \mathrm{H},-0.8754511395,-3.4253441276,-1$. $2937703171 \backslash \mathrm{H},-0.8754298137,-3.4253924066,1.2937742106 \backslash \mathrm{H},-1.5423717859$, $-2.044629875,-2.1818499086 \backslash \mathrm{H},-1.5423518887,-2.0447195651,2.1819181928 \backslash$ $C, 3.5660471779,-1.1876805108,0.0000940343 \backslash 0,-0.5885824702,3.3722428645$ $,-0.0001403249 \backslash 0,1.6549753112,3.4508054222,-0.0001404968 \backslash \mathrm{H}, 1.454220358$ $5,4.4067093268,-0.0002422114 \backslash 0,3.7732601715,-2.3809387414,0.0000958811$ $\backslash 0,4.53069248,-0.2500822191,0.0000649867 \backslash \mathrm{H}, 5.3892293391,-0.7155733148$, $0.0000648912 \backslash \mathrm{H}, 1.3116233751,-2.5459732554,0.0000859305 \backslash \backslash$ Version=IA32LG03RevC.02 State $=1-\mathrm{A} \backslash \mathrm{HF}=-973.7259819 \backslash \mathrm{S} 2=0 . \backslash \mathrm{S} 2-1=0 . \backslash \mathrm{S} 2 \mathrm{~A}=0 . \backslash \mathrm{RMSD}=9.335 \mathrm{e}-$ $09 \backslash \mathrm{RMSF}=1.672 e-05 \backslash \mathrm{Dipole}=-1.5698077,-0.3657219,-0.0000026 \backslash \mathrm{PG}=\mathrm{C} 01 \quad[\mathrm{X}(\mathrm{C} 1$ $4 \mathrm{H} 16 \mathrm{~N} 105)] \backslash \backslash 0$

\section{4,6-dicarboxy-TMIO-}

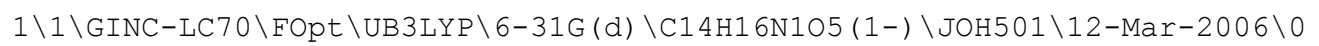
\\\# UB3LYP/6-31G* OPT FREQ=NORAMAN MAXDISK=805306368\\13cctmio-_2\\-1, $1 \backslash 0,-3.6711017454,-1.1196300811,-0.214722774 \backslash \mathrm{N},-2.391495266,-0 . \overline{7} 949557$ $199,-0.3550687928 \backslash C,-1.9764859962,0.5980688967,-0.0474483049 \backslash C,-1.3853$ $040801,-1.8140182331,-0.0389782627 \backslash C,-0.4665147813,0.3971076947,-0.072$ $6994929 \backslash \mathrm{C},-0.1432377154,-0.9691773776,-0.076015572 \backslash \mathrm{C}, 0.6001652083,1.35$ $09416627,-0.0069764662 \backslash \mathrm{C}, 1.1612774252,-1.4371722208,-0.0484427455 \backslash \mathrm{C}, 0$. $3987354539,2.8014535756,0.0811224871 \backslash C, 1.9262841319,0.8674018596,-0.00$ 
$17910464 \backslash \mathrm{C}, 2.2233957993,-0.4930668126,-0.0196210895 \backslash \mathrm{H}, 2.7501779978,1.5$ $68951909,0.0347875215 \backslash \mathrm{C},-1.448305113,-2.9011003421,-1.1308847799 \backslash \mathrm{C},-1$. $6398081993,-2.4669121479,1.3460566279 \backslash \mathrm{C},-2.5586075119,1.4828659789,-1$. $1716634476 \backslash \mathrm{C},-2.524729319,1.0863318632,1.3200527707 \backslash \mathrm{H},-3.6319729145,1$. $271841687,-1.2260873499 \backslash \mathrm{H},-3.6000157386,0.8842674961,1.3397212928 \backslash \mathrm{H},-2$ $.1106783451,1.2074766109,-2.1336506661 \backslash \mathrm{H},-2.0597180461,0.5353195945,2$. $1464708614 \backslash \mathrm{H},-2.3932880993,2.5455878033,-0.9837910553 \backslash \mathrm{H},-2.3378450225$, $2.1538189477,1.4608225568 \backslash \mathrm{H},-2.4771704844,-3.2742628433,-1.1692342374 \backslash$ $\mathrm{H},-2.7138181106,-2.6800137644,1.3941765636 \backslash \mathrm{H},-0.7675683727,-3.73678438$ $18,-0.92477168 \backslash \mathrm{H},-1.0714683096,-3.3946814754,1.4859176384 \backslash \mathrm{H},-1.2020904$ $227,-2.4714903617,-2.1077644392 \backslash \mathrm{H},-1.3842701016,-1.7800227117,2.159936$ $3445 \backslash \mathrm{C}, 3.6360914464,-0.8866890916,0.0054872752 \backslash 0,-0.6429529504,3.42035$ $85628,0.269841959 \backslash 0,1.5690217816,3.5229648906,-0.0613651111 \backslash \mathrm{H}, 1.272708$ $9723,4.4434717352,0.0495222993 \backslash 0,4.6095412393,-0.1439759473,0.03229577$ $56 \backslash 0,3.8212986411,-2.2490047764,-0.0060307034 \backslash \mathrm{H}, 4.7896499785,-2.350556$ $5568,0.0118946822 \backslash \mathrm{H}, 1.3736376577,-2.5001466735,-0.0418096717 \backslash \backslash$ Version $=$ IA32L-G03RevC. 02 \State $=1-A \backslash H F=-973.9877275 \backslash S 2=0 . \backslash S 2-1=0 . \backslash S 2 A=0 . \backslash R M S D=6$ $.156 \mathrm{e}-09 \backslash \mathrm{RMSF}=1.409 \mathrm{e}-05 \backslash \mathrm{Dipole}=1.9504132,-0.0266701,0.1946589 \backslash \mathrm{PG}=\mathrm{C} 01 \quad[$ $\mathrm{X}(\mathrm{C} 14 \mathrm{H} 16 \mathrm{~N} 105)] \backslash \backslash \mathrm{C}$

\section{4,5-dicarboxy-TMIO• (32)}

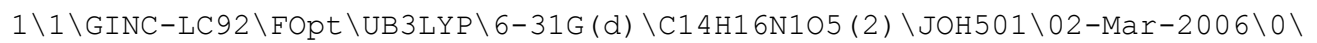
$\backslash \#$ UB3LYP/6-31G* OPT FREQ=NORAMAN MAXDISK=134217728\\12cctmio $1 \backslash \backslash 0,2 \backslash 0$ $,-3.7938244404,-0.7841118103,-0.0679172527 \backslash N,-2.5208093533,-0.76159401$ $88,-0.0841439715 \backslash \mathrm{C},-1.7539793098,0.5124034927,0.0784232988 \backslash \mathrm{C},-1.718762$ $0643,-2.0100344633,0.0020340058 \backslash C,-0.3301952796,-0.0281010833,0.038324$ $7601 \backslash \mathrm{C},-0.3221362797,-1.4320354241,0.0178609492 \backslash \mathrm{C}, 0.8771050523,0.67803$ $66835,0.0076794671 \backslash \mathrm{C}, 0.8732826655,-2.1437158052,-0.0015582685 \backslash \mathrm{C}, 0.8705$ $164246,2.1856930756,-0.0384728037 \backslash \mathrm{C}, 2.0901375953,-0.0449049832,-0.0018$ $996365 \backslash \mathrm{C}, 2.0772293664,-1.4462617216,-0.0012043996 \backslash \mathrm{C}, 3.3825821139,0.690$ $7169065,0.0456781699 \backslash \mathrm{C},-2.0071634914,-2.8801307871,-1.2339704967 \backslash \mathrm{C},-2$. $0732006023,-2.7711563806,1.2948890351 \backslash \mathrm{C},-2.1226274782,1.4509080419,-1$. $0827257241 \backslash \mathrm{C},-2.1293026204,1.1441112789,1.4349955214 \backslash \mathrm{H},-3.2114739167,1$ $.5518511484,-1.1123130607 \backslash \mathrm{H},-3.2100566132,1.3137212476,1.4548188873 \backslash \mathrm{H}$, $-1.782501537,1.0477407861,-2.0416373956 \backslash \mathrm{H},-1.867825117,0.4755534696,2$. $2616902263 \backslash \mathrm{H},-1.6845096873,2.4422660492,-0.9416389199 \backslash \mathrm{H},-1.6019617231$, $2.0891266201,1.5775655753 \backslash \mathrm{H},-3.0787444838,-3.0963338731,-1.2757723983 \backslash$ $\mathrm{H},-3.1450796557,-2.9899092904,1.2983985306 \backslash \mathrm{H},-1.4586558533,-3.82657574$ $35,-1.1802176658 \backslash \mathrm{H},-1.5184222757,-3.7138555345,1.3516580382 \backslash \mathrm{H},-1.72012$ $90153,-2.3610422747,-2.1535292134 \backslash \mathrm{H},-1.8357210513,-2.1760721832,2.1820$ $195207 \backslash 0,0.5412559016,2.9089153788,0.874132166 \backslash 0,1.1861711246,2.640577$ $1458,-1.2707156079 \backslash \mathrm{H}, 1.2040725816,3.6146682191,-1.2027862721 \backslash \mathrm{H}, 0.87312$ $42415,-3.2300198653,-0.0153597144 \backslash 0,3.501967906,1.8820446894,0.2510231$ $654 \backslash 0,4.4576130105,-0.1135868116,-0.1493524979 \backslash \mathrm{H}, 5.2372847136,0.466794$ $0026,-0.0704969398 \backslash \mathrm{H}, 3.0198802937,-1.9806363667,-0.0010744508 \backslash \backslash$ Version $=I A 32 \mathrm{~L}-\mathrm{G} 03 \mathrm{RevC} .02 \backslash$ State $=2-\mathrm{A} \backslash \mathrm{HF}=-973.9683107 \backslash \mathrm{S} 2=0.753756 \backslash \mathrm{S} 2-1=0 . \backslash \mathrm{S} 2 \mathrm{~A}=0$. $75001 \backslash \mathrm{RMSD}=4.760 \mathrm{e}-09 \backslash \mathrm{RMSF}=9.022 \mathrm{e}-06 \backslash \mathrm{Dipole}=1.0950288,-0.9361721,-0.458$ $3813 \backslash \mathrm{PG}=\mathrm{C} 01 \quad[\mathrm{X}(\mathrm{C} 14 \mathrm{H} 16 \mathrm{~N} 105)] \backslash \backslash \mathrm{e}$

\section{4,5-dicarboxy-TMIO+}

$1 \backslash 1 \backslash G I N C-L C 79 \backslash F O p t \backslash U B 3 L Y P \backslash 6-31 G(d) \backslash C 14 H 16 N 105$ (1+) \JOH501\18-Mar-2006\0

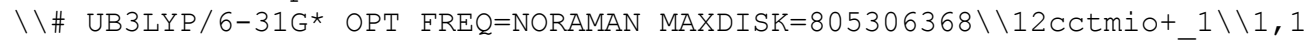
$\backslash 0,-3.6990265933,0.8793091769,-0.175379677 \backslash \mathrm{N},-2.6176633141,0.39 \overline{1} 442175$ $,-0.0702463593 \backslash C,-1.3492788033,1.2163076507,0.0514904648 \backslash C,-2.40312289$ $79,-1.1088939644,-0.0195129031 \backslash C,-0.3060201482,0.1115851702,0.01070605$ $48 \backslash C,-0.895081793,-1.1601000935,0.0126678301 \backslash C, 1.0877417356,0.24449785$ $74,-0.0133942059 \backslash \mathrm{C},-0.1205008952,-2.3150148524,0.0185109699 \backslash \mathrm{C}, 1.715733$ $3019,1.6136846463,-0.1175789426 \backslash \mathrm{C}, 1.8716208447,-0.926295888,0.02908335$ $79 \backslash C, 1.2663601168,-2.1886405116,0.0406873904 \backslash C, 3.3596729554,-0.8073566$ $184,0.1828177359 \backslash \mathrm{C},-3.0396410329,-1.7063119501,-1.293996113 \backslash \mathrm{C},-3.10010$ $68951,-1.6324886035,1.2580967527 \backslash C,-1.3386813638,2.2424410395,-1.09734$ $52821 \backslash \mathrm{C},-1.4650626602,1.9146790687,1.4390998797 \backslash \mathrm{H},-2.2570488864,2.8349$ $975024,-1.083616725 \backslash \mathrm{H},-2.3114417003,2.6068195385,1.4395450829 \backslash \mathrm{H},-1.228$ 
$6457218,1.7537575271,-2.0692865839 \backslash \mathrm{H},-1.5784341164,1.186896572,2.24723$ $93278 \backslash \mathrm{H},-0.4949705568,2.9161055463,-0.9391412072 \backslash \mathrm{H},-0.5281948063,2.456$ $2285588,1.5885722353 \backslash \mathrm{H},-4.1166752662,-1.5202313711,-1.3096811025 \backslash \mathrm{H},-4$. $174170352,-1.4313379488,1.2191675685 \backslash \mathrm{H},-2.8704798667,-2.7863769455,-1$. $2769211118 \backslash \mathrm{H},-2.9470864399,-2.7144772955,1.2993513528 \backslash \mathrm{H},-2.5804151018$, $-1.3019273798,-2.1999499777 \backslash \mathrm{H},-2.6723762088,-1.1917763958,2.1621885315$ $\backslash 0,1.4913610515,2.5237605382,0.6516312635 \backslash 0,2.4485913281,1.7309293272$, $-1.2329942753 \backslash \mathrm{H}, 2.8809239352,2.6077223487,-1.21183076 \backslash \mathrm{H},-0.5772515054$, $-3.300211852,0.0156037508 \backslash 0,3.9133736309,0.1866230551,0.5970885666 \backslash 0,3$ $.9999082556,-1.9424885518,-0.1513516744 \backslash \mathrm{H}, 4.9509632312,-1.7980677983,0$ $.0182515198 \backslash \mathrm{H}, 1.8934903908,-3.0718419009,0.07228105 \backslash \backslash$ Version=IA32L-G03 RevC.02\State $=1-\mathrm{A} \backslash \mathrm{HF}=-973.7138424 \backslash \mathrm{S} 2=0 . \backslash \mathrm{S} 2-1=0 . \backslash \mathrm{S} 2 \mathrm{~A}=0 . \backslash \mathrm{RMSD}=8.953 e-09 \backslash$ $\mathrm{RMSF}=8.192 \mathrm{e}-06 \backslash \mathrm{Dipole}=-2.4622919,-1.0487593,-0.4931441 \backslash \mathrm{PG}=\mathrm{C} 01 \quad[\mathrm{X}(\mathrm{C} 14 \mathrm{H} 1$ $6 \mathrm{~N} 105)] \backslash \backslash @$

\section{4,5-dicarboxy-TMIO-}

$1 \backslash 1 \backslash G I N C-L C 21 \backslash F O p t \backslash U B 3 L Y P \backslash 6-31 G(d) \backslash C 14 H 16 N 105$ (1-) \JOH501 \09-Mar-2006\0

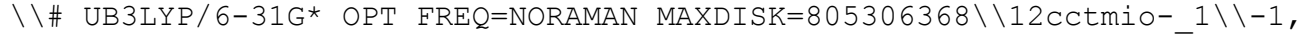
$1 \backslash 0,-3.7850554031,0.8974129461,-0.1667381947 \backslash \mathrm{N},-2.5718786425,0 . \overline{3} 703964$ $627,-0.3549906262 \backslash \mathrm{C},-1.3977817381,1.1937581474,0.0347738301 \backslash \mathrm{C},-2.40479$ $79165,-1.0627355032,-0.0586718717 \backslash \mathrm{C},-0.3110902775,0.1365077205,-0.0267$ $579701 \backslash C,-0.9000126485,-1.1349654841,-0.1029688374 \backslash C, 1.0845975833,0.27$ $3269654,-0.0120546055 \backslash \mathrm{C},-0.1092608372,-2.2919314619,-0.1273419228 \backslash \mathrm{C}, 1$. $7112473725,1.6192630484,-0.0828973982 \backslash \mathrm{C}, 1.8955498793,-0.9133405802,-0$. $0111779817 \backslash \mathrm{C}, 1.2742382196,-2.1720639634,-0.0788602489 \backslash \mathrm{C}, 3.3387183056,-$ $0.8377902684,0.2233775972 \backslash \mathrm{C},-3.1230463884,-1.861145903,-1.1673585027 \backslash \mathrm{C}$ $,-3.008872367,-1.4785299687,1.3144238031 \backslash C,-1.2928058417,2.318698721,-$ $1.0147146569 \backslash \mathrm{C},-1.5721805949,1.8134311559,1.4445078471 \backslash \mathrm{H},-2.2868243565$ $, 2.7736534435,-1.0821879564 \backslash \mathrm{H},-2.585158097,2.2283834093,1.4805888127 \backslash \mathrm{H}$ $,-1.0414613679,1.9028037105,-1.9970874082 \backslash \mathrm{H},-1.4865027488,1.0475699593$ $, 2.2235471937 \backslash \mathrm{H},-0.557737426,3.0809099903,-0.7406863284 \backslash \mathrm{H},-0.826309335$ $3,2.5866764631,1.6420261583 \backslash \mathrm{H},-4.1694622189,-1.53904558,-1.1881924478 \backslash$ $\mathrm{H},-4.0057584182,-1.0304001591,1.3741024303 \backslash \mathrm{H},-3.084334084,-2.944752551$ $3,-0.9927848647 \backslash \mathrm{H},-3.0894223398,-2.5681044652,1.4203977966 \backslash \mathrm{H},-2.675548$ $5466,-1.639011146,-2.1419806152 \backslash \mathrm{H},-2.4068601867,-1.0973848758,2.146025$ $958 \backslash 0,1.5098243106,2.5660277562,0.6573868128 \backslash 0,2.5350940533,1.74674432$ $82,-1.1656720042 \backslash \mathrm{H}, 2.9447652071,2.6242378964,-1.0539938108 \backslash \mathrm{H},-0.571923$ $0217,-3.2750209105,-0.1710737663 \backslash 0,3.9753759783,0.1256193135,0.6320586$ $777 \backslash 0,4.0045934244,-2.0210500262,-0.031931252 \backslash \mathrm{H}, 4.9221452057,-1.816099$ $6462,0.2208773996 \backslash \mathrm{H}, 1.8978668216,-3.059777206,-0.0611509737 \backslash \backslash$ Version=I A32L-G03RevC.02 $\backslash$ State $=1-A \backslash H F=-973.9756686 \backslash S 2=0 . \backslash S 2-1=0 . \backslash S 2 A=0 . \backslash R M S D=4$. $858 e-09 \backslash \mathrm{RMSF}=5.277 e-06 \backslash \mathrm{Dipole}=2.063891,-1.2934343,-0.2302914 \backslash \mathrm{PG}=\mathrm{C} 01 \quad[\mathrm{X}$ (C14H16N105) ] \\@

\section{5,6-dicarboxy-TMIO•(33)}

$1 \backslash 1 \backslash G I N C-L C 98 \backslash F O p t \backslash U B 3 L Y P \backslash 6-31 G(d) \backslash C 14 H 16 N 105$ (2) \JOH501\02-Mar-2006\0 $\backslash \#$ UB3LYP/6-31G* OPT FREQ=NORAMAN MAXDISK=134217728\\23cctmio $2 \backslash \backslash 0,2 \backslash 0$ $,-4.2119810438,0.0056010636,0.0239083059 \backslash N,-2.9386598425,0.00 \overline{6} 9217773$, $0.0170227933 \backslash C,-2.1477152902,1.2706843577,0.0601648437 \backslash C,-2.1459812049$ $,-1.2557674745,-0.0219667066 \backslash C,-0.7436773756,0.7080652758,0.0317669926$ $\backslash C,-0.7423201417,-0.690040303,-0.0124015981 \backslash C, 0.4574046912,1.410835169$ $5,0.0430245087 \backslash \mathrm{C}, 0.4594373955,-1.390290388,-0.0257501656 \backslash \mathrm{C}, 1.671425534$ $7,0.7184558204,0.0018508827 \backslash C, 1.6751780586,-0.6927749349,-0.0091523353$ $\backslash \mathrm{C}, 2.9084290901,1.5631901316,-0.0695048226 \backslash \mathrm{C},-2.4823101213,-2.03033217$ $43,-1.3091971983 \backslash \mathrm{C},-2.4726517011,-2.1027754414,1.2214396613 \backslash \mathrm{C},-2.48764$ $66464,2.128205821,-1.1723886015 \backslash \mathrm{C},-2.4708241938,2.0337941902,1.3578005$ $226 \backslash \mathrm{H},-3.5622768666,2.3331760606,-1.1812399399 \backslash \mathrm{H},-3.5441358297,2.24285$ $25803,1.3936251979 \backslash \mathrm{H},-2.2239411205,1.608172966,-2.0986337876 \backslash \mathrm{H},-2.2000$ $691397,1.443696957,2.2387746738 \backslash \mathrm{H},-1.9463400012,3.0797646603,-1.141329$ $6968 \backslash \mathrm{H},-1.9236273995,2.9816724423,1.3938263827 \backslash \mathrm{H},-3.5559909191,-2.2392$ $079827,-1.3330000861 \backslash \mathrm{H},-3.5465631642,-2.3107234508,1.2424340654 \backslash \mathrm{H},-1.9$ $36241456,-2.9791653364,-1.3419526911 \backslash \mathrm{H},-1.929048942,-3.0532644214,1.19$ $43612953 \backslash \mathrm{H},-2.2200276561,-1.4481587297,-2.198042173 \backslash \mathrm{H},-2.201505959,-1$. 
$5736690091,2.1403159816 \backslash \mathrm{H}, 0.4740196869,-2.4749041961,-0.0281162069 \backslash 0,3$ $.0993152093,2.5439525262,0.6158948003 \backslash 0,3.7409943011,1.1810261984,-1.0$ $626876426 \backslash \mathrm{H}, 4.5173977726,1.7696375745,-1.0058234034 \backslash \mathrm{C}, 2.9511812574,-1$. $4508950095,0.1424661583 \backslash 0,3.9514414176,-1.0337359536,0.6857833055 \backslash 0,2$. $8684394943,-2.7114511698,-0.3554414229 \backslash \mathrm{H}, 3.7268119053,-3.1295578556,-0$ $.1551628114 \backslash \mathrm{H}, 0.4769068437,2.4959537387,0.0722320261 \backslash \backslash$ Version=IA32L-G0 $3 R e v C .02 \backslash$ State $=2-A \backslash H F=-973.97042 \backslash \mathrm{S} 2=0.75378 \backslash \mathrm{S} 2-1=0 . \backslash \mathrm{S} 2 \mathrm{~A}=0.750011 \backslash \mathrm{RMSD}=$ $7.030 \mathrm{e}-09 \backslash \mathrm{RMSF}=1.624 \mathrm{e}-05 \backslash \mathrm{Dipole}=0.2392904,-0.7063043,-0.5764904 \backslash \mathrm{PG}=\mathrm{C} 01$

$[\mathrm{X}(\mathrm{C} 14 \mathrm{H} 16 \mathrm{~N} 105)] \backslash \backslash \mathrm{Q}$

\section{5,6-dicarboxy-TMIO+}

$1 \backslash 1 \backslash$ GINC-LC112\FOpt \UB3LYP \6-31G (d) \C14H16N1O5 (1+) \JOH501\18-Mar-2006\

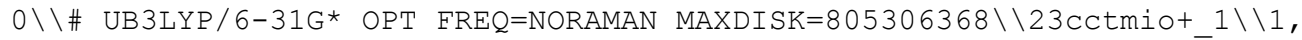
$1 \backslash 0,-4.1456406511,-0.000005545,-0.0000263193 \backslash N,-2.9555954893,-0.000003$ $6725,-0.000013776 \backslash \mathrm{C},-2.1319227256,-1.2814933321,-0.0331522137 \backslash \mathrm{C},-2.131$ $927413,1.2814883808,0.0331439015 \backslash \mathrm{C},-0.7395974498,-0.6989013867,-0.0139$ $439768 \backslash C,-0.739599999,0.6989014388,0.013935033 \backslash C, 0.4573789203,-1.40261$ $20614,-0.013980265 \backslash C, 0.4573739425,1.4026162036,0.0139764959 \backslash C, 1.671662$ $785,-0.7045274392,0.0109860116 \backslash \mathrm{C}, 1.6716603501,0.704535734,-0.010986323$ $4 \backslash \mathrm{C}, 2.8998009398,-1.5699828567,0.1108183226 \backslash \mathrm{C},-2.5019256871,2.02757773$ $52,1.3354594694 \backslash \mathrm{C},-2.5128713013,2.1034029679,-1.2185065113 \backslash \mathrm{C},-2.512866$ $3523,-2.1033936189,1.2185091585 \backslash C,-2.5019157506,-2.027600137,-1.335457$ $7571 \backslash \mathrm{H},-3.5716917698,-2.3748026374,1.1952268736 \backslash \mathrm{H},-3.5584283521,-2.308$ $8952671,-1.3330641372 \backslash \mathrm{H},-2.2880850372,-1.5612614604,2.1408673844 \backslash \mathrm{H},-2$. $2782259069,-1.427549264,-2.2214392813 \backslash \mathrm{H},-1.9153440246,-3.0192508861,1$. $2062308928 \backslash \mathrm{H},-1.8948463705,-2.9362669511,-1.3775657673 \backslash \mathrm{H},-3.5584386141$ $, 2.3088718625,1.3330653976 \backslash \mathrm{H},-3.571698478,2.3748049869,-1.1952253581 \backslash \mathrm{H}$ $,-1.8948571989,2.9362444715,1.3775825447 \backslash \mathrm{H},-1.9153549298,3.0192639096$, $-1.2062123533 \backslash \mathrm{H},-2.2782388266,1.4275150258,2.2214336851 \backslash \mathrm{H},-2.288082303$ $3,1.5612850833,-2.1408713043 \backslash \mathrm{H}, 0.4883005494,2.4876347646,0.025525466 \backslash 0$ $, 2.9832471549,-2.6533353926,-0.4211458057 \backslash 0,3.830269251,-1.0490541862$, $0.9279961661 \backslash \mathrm{H}, 4.58217827,-1.6729003709,0.9450135313 \backslash \mathrm{C}, 2.8997955117,1$. $5699953234,-0.1108165155 \backslash 0,2.9832397932,2.6533461925,0.4211515926 \backslash 0,3$. $8302846295,1.0490521983,-0.9279613671 \backslash \mathrm{H}, 4.5821966307,1.6728951117,-0.9$ $449658361 \backslash \mathrm{H}, 0.4883087425,-2.4876305178,-0.0255284178 \backslash \backslash$ Version=IA32L-G0 $3 R e v C .02 \backslash$ State $=1-A \backslash H F=-973.7116083 \backslash S 2=0 . \backslash S 2-1=0 . \backslash S 2 A=0 . \backslash R M S D=6.755 e-09$ $\backslash \mathrm{RMSF}=7.703 e-06 \backslash \mathrm{Dipole}=-3.0036384,-0.000005,0.0000076 \backslash \mathrm{PG}=\mathrm{C} 01 \quad[\mathrm{X}(\mathrm{C} 14 \mathrm{H} 16$ $\mathrm{N} 105)] \backslash \backslash @$

\section{5,6-dicarboxy-TMIO-}

$1 \backslash 1 \backslash G I N C-L C 119 \backslash$ FOpt \UB3LYP \6-31G (d) \C14H16N1O5 (1-) \JOH501\12-Mar-2006\ $0 \backslash \backslash \#$ UB3LYP/6-31G* OPT FREQ=NORAMAN MAXDISK=805306368\\23cctmio- $2 \backslash \backslash-1$ $, 1 \backslash 0,-4.2189840418,0.0065045775,-0.1393483071 \backslash \mathrm{N},-2.8991122058,0 . \overline{0} 04688$ $0962,-0.4240497829 \backslash \mathrm{C},-2.1536761448,-1.2366175846,-0.0753268253 \backslash \mathrm{C},-2.14$ $04455516,1.2223631844,-0.0177264559 \backslash C,-0.7413403745,-0.6965685272,-0.1$ $245614143 \backslash C,-0.7280606834,0.706440329,-0.0784006979 \backslash C, 0.46894786,-1.39$ $86999828,-0.0908400216 \backslash \mathrm{C}, 0.4663904111,1.4074816264,-0.0608343934 \backslash \mathrm{C}, 1.6$ $827202885,-0.7093218461,-0.0341191096 \backslash C, 1.7019574383,0.7097031302,-0.0$ $499855769 \backslash \mathrm{C}, 2.9049514918,-1.5507539333,0.1225845875 \backslash \mathrm{C},-2.5478880931,1$. $6990944901,1.3979946278 \backslash \mathrm{C},-2.4610204206,2.3194214147,-1.0464789124 \backslash \mathrm{C},-$ $2.5434474898,-1.8163195783,1.3134669551 \backslash C,-2.4806665268,-2.2876258535$, $-1.1533308811 \backslash \mathrm{H},-3.6356831154,-1.7799792173,1.3718940616 \backslash \mathrm{H},-3.56587535$ $93,-2.4363176688,-1.1601776957 \backslash \mathrm{H},-2.1328356206,-1.2134087721,2.1307728$ $438 \backslash \mathrm{H},-2.1783347729,-1.9204685817,-2.1395748551 \backslash \mathrm{H},-2.1964771423,-2.849$ $5652906,1.4434689296 \backslash \mathrm{H},-1.9869813073,-3.2500393512,-0.9613325889 \backslash \mathrm{H},-3$. $6309713002,1.5254206882,1.453002504 \backslash \mathrm{H},-3.5491183291,2.4443327671,-1.06$ $31607338 \backslash \mathrm{H},-2.3212490061,2.7585996937,1.5698138563 \backslash \mathrm{H},-1.9885614019,3.2$ $784935202,-0.795853313 \backslash \mathrm{H},-2.0566093252,1.1132405334,2.1821075028 \backslash \mathrm{H},-2$. $13712051,2.0082824651,-2.0452988872 \backslash \mathrm{H}, 0.4753924933,2.4920481207,-0.066$ $2395594 \backslash 0,3.1962141434,-2.5084019353,-0.5686293317 \backslash 0,3.6338723881,-1.2$ $266768058,1.2248640576 \backslash \mathrm{H}, 4.4098104668,-1.8158763731,1.1890872921 \backslash \mathrm{C}, 2.9$ $688670978,1.4325916654,-0.1820566202 \backslash 0,4.0544380999,0.9666902978,-0.50$ $8204167 \backslash 0,2.8557195105,2.7829725588,0.0591640219 \backslash \mathrm{H}, 3.7506128143,3.1218$ 
$034968,-0.1208900234 \backslash \mathrm{H}, 0.4839702387,-2.485223456,-0.0843526136 \backslash \backslash$ Versio $\mathrm{n}=\mathrm{IA} 32 \mathrm{~L}-\mathrm{G} 03 \mathrm{RevC} .02 \backslash \mathrm{State}=1-\mathrm{A} \backslash \mathrm{HF}=-973.977825 \backslash \mathrm{S} 2=0 . \backslash \mathrm{S} 2-1=0 . \backslash \mathrm{S} 2 \mathrm{~A}=0 . \backslash \mathrm{RMSD}=$ $9.454 \mathrm{e}-09 \backslash \mathrm{RMSF}=4.848 \mathrm{e}-06 \backslash \mathrm{Dipole}=2.4451825,0.5067347,0.7358672 \backslash \mathrm{PG}=\mathrm{C} 01 \quad[$ $\mathrm{X}(\mathrm{C} 14 \mathrm{H} 16 \mathrm{~N} 105)] \backslash \backslash \mathrm{e}$

\section{5-CH $\mathrm{CN}-\mathrm{TMIO} \bullet(34)$}

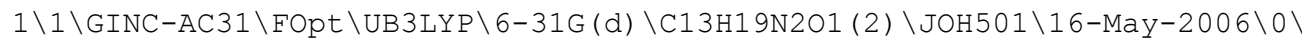
\\# UB3LYP/6-31G* OPT FREQ=noraman maxdisk=402653184 \\cntmio $1 \backslash \backslash 0,2 \backslash \mathrm{N}, 1$ $.8011772921,0.2669044124,0.0536265892 \backslash \mathrm{C}, 1.2921795458,-1.133 \overline{4} 998573,-0$. $0019849373 \backslash \mathrm{C},-0.2001182516,-0.8784017527,-0.0067623138 \backslash \mathrm{C},-0.4959811371$ $, 0.4901466642,0.0148197331 \backslash \mathrm{C},-1.8153996777,0.9338956072,0.0140973368 \backslash \mathrm{C}$ $,-2.8625896641,0.0021941208,-0.0081608353 \backslash \mathrm{C},-1.2331013638,-1.812387569$ $8,-0.0249848511 \backslash \mathrm{C},-2.5564270012,-1.3654143912,-0.0211536607 \backslash \mathrm{C}, 0.760842$ $2272,1.3351185787,0.0376446322 \backslash \mathrm{H},-2.0576660038,1.992258317,0.042614194$ $6 \backslash \mathrm{C},-4.3076153987,0.4651229827,-0.0481491101 \backslash \mathrm{H},-1.0205669325,-2.878778$ $5784,-0.0336002712 \backslash \mathrm{H},-3.3661676744,-2.0921151093,-0.0264787629 \backslash 0,3.047$ $2928474,0.5340889624,0.0589814425 \backslash \mathrm{C}, 0.8991398016,2.1966298711,1.305262$ $407 \backslash \mathrm{C}, 0.9380534165,2.1995026406,-1.2241209018 \backslash \mathrm{C}, 1.8015161653,-1.809187$ $4683,-1.2886273688 \backslash \mathrm{C}, 1.7828634258,-1.9001952729,1.2391302625 \backslash \mathrm{H}, 0.20344$ $50607,3.0117562693,-1.2391974347 \backslash \mathrm{H}, 1.9437932549,2.6306725033,-1.230765$ $9706 \backslash \mathrm{H}, 0.8088227559,1.5996917686,-2.1305741443 \backslash \mathrm{H}, 0.1651120899,3.009545$ $2444,1.2996135068 \backslash \mathrm{H}, 0.7420907571,1.5949255041,2.20600127 \backslash \mathrm{H}, 1.904346945$ $4,2.6275059816,1.3438174386 \backslash \mathrm{H}, 1.4701730307,-2.8524369375,-1.3324433933$ $\backslash \mathrm{H}, 1.4283571838,-1.2903599474,-2.1772011445 \backslash \mathrm{H}, 2.895451697,-1.784453953$ $1,-1.3028946851 \backslash \mathrm{H}, 1.4516889398,-2.9437503174,1.2029510283 \backslash \mathrm{H}, 2.87646032$ $27,-1.8779155196,1.2706863016 \backslash \mathrm{H}, 1.397186131,-1.4460573204,2.1571916174$ $\backslash \mathrm{H},-4.9561180428,-0.3789827186,0.2464628943 \backslash \mathrm{N},-4.5097330485,1.68480234$ $64,0.7422132628 \backslash \mathrm{H},-4.5794399346,0.7089624886,-1.085112519 \backslash \mathrm{H},-5.4684675$ $698,2.0103136056,0.6332543709 \backslash \mathrm{H},-4.38331119,1.4765788452,1.731824016 \backslash \backslash$ Version=IA $64 \mathrm{~L}-\mathrm{G} 03$ RevD. $01 \backslash$ State $=2-\mathrm{A} \backslash \mathrm{HF}=-691.4962299 \backslash \mathrm{S} 2=0.753787 \backslash \mathrm{S} 2-1=0$. $\backslash \mathrm{S} 2 \mathrm{~A}=0.75001 \backslash \mathrm{RMSD}=6.437 \mathrm{e}-09 \backslash \mathrm{RMSF}=3.558 \mathrm{e}-06 \backslash \mathrm{Thermal}=0 . \backslash \mathrm{Dipole}=-1.664381$ $4,-0.4208384,0.1743476 \backslash \mathrm{PG}=\mathrm{C} 01 \quad[\mathrm{X}(\mathrm{C} 13 \mathrm{H} 19 \mathrm{~N} 2 \mathrm{O} 1)] \backslash \backslash \mathrm{C}$

\section{5- $\mathrm{CH}_{2} \mathrm{CN}-\mathrm{TMIO}+$}

$1 \backslash 1 \backslash G I N C-A C 27 \backslash F O p t \backslash U B 3 L Y P \backslash 6-31 G(d) \backslash C 13 H 19 N 2 O 1(1+) \backslash J O H 501 \backslash 16-M a y-2006 \backslash 0$ $\backslash \backslash \#$ UB3LYP/6-31G* OPT FREQ=noraman maxdisk=402653184 \\cntmio+ $3 \backslash \backslash 1,1 \backslash \mathrm{N}$ $,-0.0139989835,-0.0175104749,0.0005908108 \backslash \mathrm{C}, 1.5087661941,-0.0 \overline{3} 14724809$ $, 0.048645222 \backslash C, 1.7875609057,1.4506735203,-0.0126773675 \backslash C, 0.6207265312$, $2.2179891073,-0.071800487 \backslash C, 0.6748159053,3.6069656515,-0.133357739 \backslash C, 1$ $.920863279,4.2450678306,-0.1341161472 \backslash C, 3.0339946424,2.0741236838,-0.0$ $179898813 \backslash \mathrm{C}, 3.0877188024,3.4663869837,-0.0825729088 \backslash \mathrm{C},-0.6300233162,1$. $3723880113,-0.0600455333 \backslash \mathrm{H},-0.2170629849,4.224315724,-0.1886055069 \backslash \mathrm{C}, 2$ $.0063993603,5.7634673021,-0.1541294704 \backslash \mathrm{H}, 3.9533531784,1.4963378196,0.0$ $206527846 \backslash \mathrm{H}, 4.056972691,3.958135046,-0.0948402587 \backslash 0,-0.6672588485,-1.0$ $13042927,0.0112125114 \backslash \mathrm{C},-1.4777920766,1.4422296537,-1.3492477106 \backslash \mathrm{C},-1$. $5085617489,1.5328499572,1.2017398285 \backslash \mathrm{C}, 1.910730934,-0.7148395488,1.375$ $8076266 \backslash \mathrm{C}, 1.9959967608,-0.843000473,-1.1718157673 \backslash \mathrm{H},-1.933924159,2.539$ $9281546,1.1770329083 \backslash \mathrm{H},-2.3236330219,0.8039595253,1.2046362195 \backslash \mathrm{H},-0.91$ $84622173,1.4356647908,2.1168838522 \backslash \mathrm{H},-1.90372771,2.4476877871,-1.40686$ $13563 \backslash \mathrm{H},-0.8658128481,1.280335365,-2.2405663502 \backslash \mathrm{H},-2.2934479338,0.7146$ $414886,-1.3205141157 \backslash \mathrm{H}, 3.0031131716,-0.7156273441,1.4268558704 \backslash \mathrm{H}, 1.530$ $4878763,-0.1656321516,2.241144966 \backslash \mathrm{H}, 1.5561737887,-1.7488712322,1.40533$ $95177 \backslash \mathrm{H}, 3.0893265933,-0.8455195029,-1.1502602862 \backslash \mathrm{H}, 1.641618859,-1.8761$ $230536,-1.1207276465 \backslash \mathrm{H}, 1.6753516583,-0.3855485386,-2.1114993019 \backslash \mathrm{H}, 2.94$ $38246533,6.0575930153,-0.6543257687 \backslash \mathrm{N}, 0.8011933473,6.3710151573,-0.717$ $0547677 \backslash \mathrm{H}, 2.0976750754,6.1102227114,0.883968309 \backslash \mathrm{H}, 0.7432559818,7.34932$ $70029,-0.4455082717 \backslash \mathrm{H}, 0.8365656592,6.3539224379,-1.7348237842 \backslash \backslash$ Version $=I A 64 \mathrm{~L}-\mathrm{G} 03$ RevD. $01 \backslash \mathrm{State}=1-\mathrm{A} \backslash \mathrm{HF}=-691.2507181 \backslash \mathrm{S} 2=0 . \backslash \mathrm{S} 2-1=0 . \backslash \mathrm{S} 2 \mathrm{~A}=0 . \backslash \mathrm{RMSD}=$ $5.433 e-09 \backslash \mathrm{RMSF}=1.564 \mathrm{e}-05 \backslash \mathrm{Thermal}=0 . \backslash \mathrm{Dipole}=-0.0908975,-0.7891553,-0.09$ $11895 \backslash P G=C 01 \quad[X(C 13 H 19 N 201)] \backslash \backslash @$ 


\section{5-CH $2 \mathrm{CN}-\mathrm{TMIO}-$}

$1 \backslash 1 \backslash G I N C-A C 30 \backslash F O p t \backslash U B 3 L Y P \backslash 6-31 G(d) \backslash C 13 H 19 N 2 O 1$ (1-) \JOH501\16-May-2006\0 $\backslash \backslash \#$ UB3LYP/6-31G* OPT FREQ=noraman maxdisk=402653184 \\cntmio- $2 \backslash \backslash-1,1 \backslash$ $\mathrm{N}, 0.0008934611,0.0639492101,-0.5313248841 \backslash \mathrm{C}, 1.4468864296,0.07 \overline{2} 3652144$, $-0.1346094637 \backslash \mathrm{C}, 1.7208095412,1.5577219725,-0.1210005611 \backslash \mathrm{C}, 0.5119944898$ $, 2.2703268816,-0.0506031883 \backslash \mathrm{C}, 0.5207308834,3.6601346484,0.0341887593 \backslash \mathrm{C}$ $, 1.7408624215,4.3646184977,0.0455910446 \backslash \mathrm{C}, 2.934840288,2.2415314844,-0$. $1023586384 \backslash C, 2.9379439076,3.6416627544,-0.0193075359 \backslash C,-0.6526916804,1$ $.3090446884,-0.0105082404 \backslash \mathrm{H},-0.4175279502,4.2115052679,0.1035618461 \backslash \mathrm{C}$, $1.7521020552,5.8797711981,0.0612771367 \backslash \mathrm{H}, 3.8764664915,1.6967235923,-0$. $1419388293 \backslash \mathrm{H}, 3.886179812,4.179944884,0.0035362338 \backslash 0,-0.6661887431,-1.0$ $989062761,-0.2310999489 \backslash \mathrm{C},-1.7936561901,1.6409698024,-0.9874672192 \backslash \mathrm{C},-$ $1.2409951359,1.1147329615,1.410409837 \backslash C, 1.6852577545,-0.6086130957,1.2$ $374755157 \backslash \mathrm{C}, 2.2003216962,-0.7112060397,-1.2232200619 \backslash \mathrm{H},-2.0862692759,1$ $.7835337014,1.6206334684 \backslash \mathrm{H},-1.5682097208,0.0643487873,1.4160472584 \backslash \mathrm{H},-$ $0.4885505004,1.2598634155,2.1934093499 \backslash \mathrm{H},-2.3801039664,2.5174227927,-0$ $.675702169 \backslash \mathrm{H},-1.3919087365,1.8126999436,-1.9922258944 \backslash \mathrm{H},-2.4471684633$, $0.7624144037,-1.0278055495 \backslash \mathrm{H}, 2.699627766,-1.0157595469,1.3441504541 \backslash \mathrm{H}$, $1.5021125528,0.0743185197,2.0744761129 \backslash \mathrm{H}, 0.9388198278,-1.4159716287,1$. $2640705976 \backslash \mathrm{H}, 3.2738186275,-0.8146940445,-1.0084675805 \backslash \mathrm{H}, 1.7431725404,-$ $1.705560529,-1.2768023538 \backslash \mathrm{H}, 2.0715205077,-0.2244618645,-2.1962602201 \backslash \mathrm{H}$ $, 2.6988374122,6.2456101439,0.4796809591 \backslash \mathrm{N}, 1.5796825841,6.5354447942,-1$ $.2593019401 \backslash \mathrm{H}, 0.9520512682,6.2599839983,0.709817682 \backslash \mathrm{H}, 2.2888063459,6.1$ $440474491,-1.8827461463 \backslash \mathrm{H}, 0.6984616988,6.1974720178,-1.6513458305 \backslash \backslash$ Ver sion=IA 64L-G03RevD. $01 \backslash$ State $=1-A \backslash H F=-691.489172 \backslash \mathrm{S} 2=0 . \backslash \mathrm{S} 2-1=0 . \backslash \mathrm{S} 2 \mathrm{~A}=0 . \backslash \mathrm{RM}$ $\mathrm{SD}=7.855 \mathrm{e}-09 \backslash \mathrm{RMSF}=4.633 \mathrm{e}-06 \backslash \mathrm{Thermal}=0 . \backslash \mathrm{Dipole}=1.4650687,2.5790244,0.09$ $11618 \backslash \mathrm{PG}=\mathrm{C} 01 \quad[\mathrm{X}(\mathrm{C} 13 \mathrm{H} 19 \mathrm{~N} 2 \mathrm{O} 1)] \backslash \backslash \mathrm{Q}$

\section{$5-\mathrm{NH}_{3}{ }^{+}-\mathrm{TMIO} \bullet(35)$}

$1 \backslash 1 \backslash$ GINC-LC10 \FOpt \UB3LYP \6-31G(d) \C12H18N2O1 $(1+, 2) \backslash J O H 501 \backslash 13-M a r-2006$

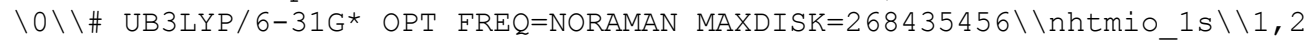
$\backslash 0,-3.131883732,-0.1292732738,0 . \backslash \mathrm{N},-1.8616494876,-0.1258116785,0 . \backslash \mathrm{C},-1$ $.0683365931,-1.3884331333,0 . \backslash C,-1.0826256814,1.1447027602,0 . \backslash C, 0.32369$ $97254,0.5861502804,0 . \backslash C, 0.3333366086,-0.8191659072,0 . \backslash C, 1.5348832232,-$ $1.5237455028,0 . \backslash C, 2.7449053056,-0.8239082157,0 . \backslash C, 2.7003640291,0.56484$ $72469,0 . \backslash \mathrm{C}, 1.5179449816,1.3009290189,0 . \backslash \mathrm{N}, 3.9978765656,1.3157092005,0$. $\backslash \mathrm{H}, 4.7958996333,0.6681711517,0 . \backslash \mathrm{H}, 3.6892005924,-1.3632804854,0 . \backslash \mathrm{H}, 1.54$ $64240013,-2.609130917,0 . \backslash \mathrm{H}, 1.5214058147,2.3886278818,0 . \backslash \mathrm{C},-1.416828366$ $, 1.953512482,-1.2675904148 \backslash \mathrm{C},-1.416828366,1.953512482,1.2675904148 \backslash \mathrm{C},-$ $1.3922841854,-2.2011560643,-1.2676774582 \backslash C,-1.3922841854,-2.2011560643$ $, 1.2676774582 \backslash \mathrm{H}, 4.0955727707,1.9179313587,-0.8287881039 \backslash \mathrm{H}, 4.0955727707$ $, 1.9179313587,0.8287881039 \backslash \mathrm{H},-2.4940290086,2.1398971666,-1.2907688293 \backslash$ $\mathrm{H},-2.4940290086,2.1398971666,1.2907688293 \backslash \mathrm{H},-0.8966089392,2.9176025829$ $,-1.2654454867 \backslash \mathrm{H},-0.8966089392,2.9176025829,1.2654454867 \backslash \mathrm{H},-1.14133684$ $56,1.4055126691,-2.1739240968 \backslash \mathrm{H},-1.1413368456,1.4055126691,2.173924096$ $8 \backslash \mathrm{H},-0.8545865464,-3.1549917385,-1.2662990731 \backslash \mathrm{H},-0.8545865464,-3.15499$ $17385,1.2662990731 \backslash \mathrm{H},-2.466327572,-2.4052353421,-1.2893040306 \backslash \mathrm{H},-2.466$ $327572,-2.4052353421,1.2893040306 \backslash \mathrm{H},-1.1262482138,-1.6487268924,-2.174$ $1930417 \backslash \mathrm{H},-1.1262482138,-1.6487268924,2.1741930417 \backslash \backslash$ Version=IA32L-G03R evC.02 \State $=2-\mathrm{A} " \backslash \mathrm{HF}=-652.5437117 \backslash \mathrm{S} 2=0.753771 \backslash \mathrm{S} 2-1=0 . \backslash \mathrm{S} 2 \mathrm{~A}=0.750011 \backslash \mathrm{RMS}$ $\mathrm{D}=8.508 \mathrm{e}-09 \backslash \mathrm{RMSF}=1.154 \mathrm{e}-05 \backslash \mathrm{Dipole}=6.5009127,1.6300993,0 . \backslash \mathrm{PG}=\mathrm{CS} \quad[\mathrm{SG}(\mathrm{C} 8 \mathrm{H}$ $4 \mathrm{~N} 2 \mathrm{O} 1), \mathrm{X}(\mathrm{C} 4 \mathrm{H} 14)] \backslash \backslash \mathrm{Q}$

\section{$5-\mathrm{NH}_{3}{ }^{+}$-TMIO+}

$1 \backslash 1 \backslash G I N C-L C 91 \backslash$ FOpt \UB3LYP \6-31G(d) \C12H18N2O1 (2+) \JOH501\13-Mar-2006\0 $\backslash \backslash \#$ UB3LYP/6-31G* OPT FREQ=NORAMAN MAXDISK=268435456\\nhtmio+_1s $\backslash \backslash 2,1 \backslash$ $0,-3.0673756641,-0.1328194533,0 . \backslash \mathrm{N},-1.8798654715,-0.128132858,0 . \backslash \mathrm{C},-1$. $0519510471,-1.4063944047,0 . \backslash \mathrm{C},-1.0673038467,1.1640812639,0 . \backslash \mathrm{C}, 0.332540$ $4729,0.5868258362,0 . \backslash C, 0.3402298341,-0.8147311938,0 . \backslash C, 1.53929366,-1.5$ $236429388,0 . \backslash C, 2.7467046964,-0.8203472356,0 . \backslash C, 2.7120925362,0.57044774$ $83,0 . \backslash \mathrm{C}, 1.5271501522,1.3052929949,0 . \backslash \mathrm{N}, 4.0111151166,1.313548013,0 . \backslash \mathrm{H}, 4$ $.8119998861,0.6657245305,0 . \backslash \mathrm{H}, 3.6865107911,-1.3664693434,0 . \backslash \mathrm{H}, 1.556486$ 
$946,-2.608931645,0 . \backslash \mathrm{H}, 1.5319616944,2.3923498023,0 . \backslash \mathrm{C},-1.4500615531,1.9$ $446700795,-1.2792979077 \backslash C_{,}-1.4500615531,1.9446700795,1.2792979077 \backslash C_{,}-1$ $.4207979806,-2.1952085873,-1.2795318015 \backslash C,-1.4207979806,-2.1952085873$, $1.2795318015 \backslash \mathrm{H}, 4.1185606845,1.9151138482,-0.8305266061 \backslash \mathrm{H}, 4.1185606845$, $1.9151138482,0.8305266061 \backslash \mathrm{H},-2.5148397961,2.1917503145,-1.2668379354 \backslash \mathrm{H}$ $,-2.5148397961,2.1917503145,1.2668379354 \backslash \mathrm{H},-0.8814755276,2.8791713105$, $-1.2883189446 \backslash \mathrm{H},-0.8814755276,2.8791713105,1.2883189446 \backslash \mathrm{H},-1.212250275$ $5,1.3842361894,-2.1873729003 \backslash \mathrm{H},-1.2122502755,1.3842361894,2.1873729003$ $\backslash \mathrm{H},-0.8288536252,-3.1150065531,-1.288197977 \backslash \mathrm{H},-0.8288536252,-3.1150065$ $531,1.288197977 \backslash \mathrm{H},-2.4797682772,-2.4656265364,-1.2644316708 \backslash \mathrm{H},-2.47976$ $82772,-2.4656265364,1.2644316708 \backslash \mathrm{H},-1.1958361145,-1.630015639,-2.18788$ $59857 \backslash \mathrm{H},-1.1958361145,-1.630015639,2.1878859857 \backslash \backslash$ Version=IA32L-G03RevC $.02 \backslash$ State $=1-A^{\prime} \backslash H F=-652.1881828 \backslash \mathrm{S} 2=0 . \backslash \mathrm{S} 2-1=0 . \backslash \mathrm{S} 2 \mathrm{~A}=0 . \backslash \mathrm{RMSD}=7.588 \mathrm{e}-09 \backslash \mathrm{RMS}$ $\mathrm{F}=9.442 e-05 \backslash \mathrm{Dipole}=4.3601215,1.4493274,0 . \backslash \mathrm{PG}=\mathrm{CS} \quad[\mathrm{SG}(\mathrm{C} 8 \mathrm{H} 4 \mathrm{~N} 2 \mathrm{O} 1), \mathrm{X}(\mathrm{C} 4 \mathrm{H} 14)$ ]$\backslash \backslash 0$

\section{5- $\mathrm{NH}_{3}{ }^{+}$-TMIO-}

$1 \backslash 1 \backslash G I N C-L C 51 \backslash F O p t \backslash U B 3 L Y P \backslash 6-31 G(d) \backslash C 12 H 18 N 201 \backslash J O H 501 \backslash 14-M a r-2006 \backslash 0 \backslash \backslash \#$

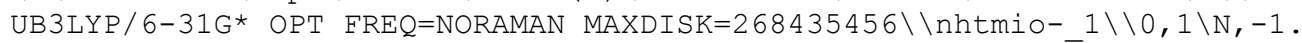
$792778825,0.264174344,-0.3525713456 \backslash C,-1.3306398375,-1.10 \overline{4} 6444299,-0.0$ $254544427 \backslash \mathrm{C}, 0.1558966039,-0.8655997652,-0.0978296584 \backslash \mathrm{C}, 0.4498201316,0$. $5016743052,-0.0947739773 \backslash C, 1.7689646861,0.9601576471,-0.0667861904 \backslash C, 2$ $.7729354586,-0.0302478137,-0.0456115663 \backslash C, 1.1846092519,-1.8252296714,-$ $0.0474194448 \backslash \mathrm{C}, 2.51536777,-1.3929995815,-0.0058381877 \backslash \mathrm{C},-0.807849467,1$ $.321307672,-0.0318844649 \backslash \mathrm{H}, 2.0056846987,2.0208072916,-0.0857037439 \backslash \mathrm{N}, 4$ $.1788367841,0.429598989,0.1100336279 \backslash \mathrm{H}, 0.9589081834,-2.8864974421,-0.0$ $362898215 \backslash \mathrm{H}, 3.3299833363,-2.1148772767,0.0531472618 \backslash 0,-3.0731107355,0$. $5454314605,-0.1605316628 \backslash \mathrm{C},-0.9160573973,2.4120189522,-1.1150274303 \backslash \mathrm{C}$, $-1.058759335,1.9573021642,1.361962192 \backslash \mathrm{C},-1.8118993996,-1.5873164695,1$. $370637826 \backslash \mathrm{C},-1.8834462806,-2.0625469861,-1.099311718 \backslash \mathrm{H},-0.5319209291,2$ $.9099066017,1.4940484825 \backslash \mathrm{H},-2.1388444672,2.1250392261,1.429284431 \backslash \mathrm{H},-0$ $.7636193839,1.28140328,2.1718735362 \backslash \mathrm{H},-0.2563047169,3.2661182716,-0.91$ $44943814 \backslash \mathrm{H},-0.6819027455,1.996386238,-2.1007167597 \backslash \mathrm{H},-1.9535226158,2.7$ $599929916,-1.1301811528 \backslash \mathrm{H},-1.6563212453,-2.6631378457,1.5150057891 \backslash \mathrm{H},-$ $1.299697793,-1.0532338224,2.1784548355 \backslash \mathrm{H},-2.8811265014,-1.3646902203,1$ $.4357559363 \backslash \mathrm{H},-1.6131231046,-3.1071666027,-0.8996606613 \backslash \mathrm{H},-2.974150566$ $6,-1.9730467589,-1.1020174469 \backslash \mathrm{H},-1.5144481289,-1.7783994443,-2.0903062$ $136 \backslash \mathrm{H}, 4.8291123546,-0.3642733753,0.0833232855 \backslash \mathrm{H}, 4.4529903279,1.0758797$ $174,-0.6404010394 \backslash \mathrm{H}, 4.3371303566,0.9466680144,1.0049173659 \backslash \backslash$ Version=IA $32 \mathrm{~L}-\mathrm{G} 03$ RevC. 02 $\backslash$ State $=1-\mathrm{A} \backslash \mathrm{HF}=-652.6500087 \backslash \mathrm{S} 2=0 . \backslash \mathrm{S} 2-1=0 . \backslash \mathrm{S} 2 \mathrm{~A}=0 . \backslash \mathrm{RMSD}=9.2$ $48 \mathrm{e}-09 \backslash \mathrm{RMSF}=1.101 \mathrm{e}-05 \backslash \mathrm{Dipole}=6.8948759,0.1006908,0.340183 \backslash \mathrm{PG}=\mathrm{C} 01 \quad[\mathrm{X}(\mathrm{C} 1$ $2 \mathrm{H} 18 \mathrm{~N} 2 \mathrm{O} 1)] \backslash \backslash \mathrm{C}$

\section{TMAO• $(36)$}

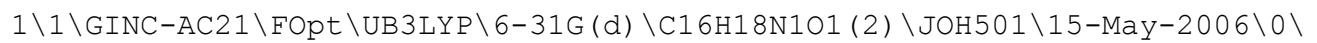

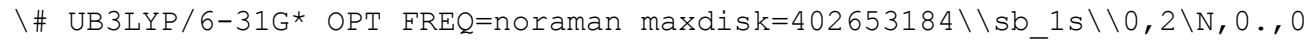
$., 0 . \backslash 0,-1.28471,0 ., 0 . \backslash C, 2.84232,0.05404,0 . \backslash C, 4.28001,-0.02859,0 . \backslash C, 4.9$ $8026,-0.06307,1.23256 \backslash \mathrm{C}, 4.98026,-0.06307,-1.23256 \backslash \mathrm{H}, 6.06538,-0.12765,1$ $.21559 \backslash \mathrm{H}, 6.06538,-0.12765,-1.21559 \backslash \mathrm{C}, 4.29669,-0.00822,2.42279 \backslash \mathrm{C}, 4.2966$ $9,-0.00822,-2.42279 \backslash \mathrm{H}, 4.83192,-0.02874,3.36811 \backslash \mathrm{H}, 4.83192,-0.02874,-3.3$ $6811 \backslash \mathrm{C}, 2.88815,0.07447,2.42451 \backslash \mathrm{C}, 2.88815,0.07447,-2.42451 \backslash \mathrm{H}, 2.37477,0$. $1211,3.37977 \backslash \mathrm{H}, 2.37477,0.1211,-3.37977 \backslash \mathrm{C}, 2.15598,0.09855,1.25082 \backslash \mathrm{C}, 2.1$ $5598,0.09855,-1.25082 \backslash \mathrm{C}, 0.63808,0.21062,1.33335 \backslash \mathrm{C}, 0.63808,0.21062,-1.3$ $3335 \backslash C, 0.22928,1.61328,1.84735 \backslash C, 0.22928,1.61328,-1.84735 \backslash C, 0.05263,-0$ $.87948,2.2592 \backslash \mathrm{C}, 0.05263,-0.87948,-2.2592 \backslash \mathrm{H}, 0.3764,-1.87299,1.93365 \backslash \mathrm{H}, 0$ $.3764,-1.87299,-1.93365 \backslash \mathrm{H}, 0.37893,-0.72949,3.29167 \backslash \mathrm{H}, 0.37893,-0.72949$, $-3.29167 \backslash \mathrm{H},-1.03708,-0.83473,2.22308 \backslash \mathrm{H},-1.03708,-0.83473,-2.22308 \backslash \mathrm{H}, 0$. $66665,2.4034,1.22867 \backslash \mathrm{H}, 0.66665,2.4034,-1.22867 \backslash \mathrm{H},-0.85996,1.70352,1.82$ $116 \backslash \mathrm{H},-0.85996,1.70352,-1.82116 \backslash \mathrm{H}, 0.57665,1.75848,2.875 \backslash \mathrm{H}, 0.57665,1.75$ $848,-2.875 \backslash \backslash$ Version=IA64L-G03RevD.01 \State $=2-A^{\prime} \backslash H F=-750.4707755 \backslash S 2=0.7$ $53764 \backslash \mathrm{S} 2-1=0 . \backslash \mathrm{S} 2 \mathrm{~A}=0.75001 \backslash \mathrm{RMSD}=5.032 \mathrm{e}-09 \backslash \mathrm{RMSF}=1.800 \mathrm{e}-05 \backslash \mathrm{Thermal}=0 . \backslash \mathrm{Dip}$ ole $=-0.2387135,0.9807209,0 . \backslash P G=C S \quad[S G(C 2 N 1 O 1), x(C 14 H 18)] \backslash \backslash @$ 


\section{TMAO+}

$1 \backslash 1 \backslash G I N C-L C 34 \backslash F O p t \backslash U B 3 L Y P \backslash 6-31 G(d) \backslash C 16 H 18 N 101(1+) \backslash J O H 501 \backslash 13-M a y-2006 \backslash 0$

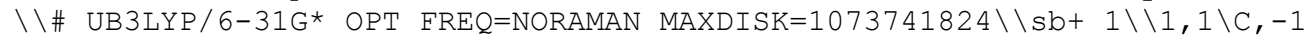
$.2349658943,-3.0249389344,-0.3664299618 \backslash \mathrm{C},-0.0193482127,-3.3677436309$, $0.1839830506 \backslash \mathrm{C},-1.5686282197,-1.6629146622,-0.5844678586 \backslash \mathrm{C},-0.63228379$ $35,-0.649485613,-0.2040475546 \backslash C,-1.0157150593,0.7206391512,-0.29855929$ $67 \backslash C,-2.2018524525,1.0662638843,-0.9269718695 \backslash C,-2.7969087043,-1.26919$ $7589,-1.1738672036 \backslash \mathrm{C},-3.0902981965,0.0647923074,-1.3719893141 \backslash \mathrm{C}, 0.6591$ $523371,-1.0313628943,0.2487837313 \backslash \mathrm{C},-0.1971562675,1.7498171796,0.48859$ $3234 \backslash \mathrm{H},-2.4935449502,2.105152848,-1.0296493564 \backslash \mathrm{H},-3.5036661005,-2.0365$ $791749,-1.4767616657 \backslash \mathrm{H},-4.0277915888,0.356060597,-1.8345647051 \backslash \mathrm{C}, 0.938$ $318069,-2.3708276594,0.4690357949 \backslash \mathrm{H},-1.9535988809,-3.792811393,-0.6379$ $591005 \backslash \mathrm{H}, 0.2278122996,-4.4088280915,0.3643801871 \backslash \mathrm{H}, 1.9183036204,-2.678$ $4013297,0.8218103564 \backslash \mathrm{C}, 1.7895680066,-0.01397358,0.2570224458 \backslash \mathrm{N}, 1.23470$ $04667,1.3936498183,0.1154361794 \backslash 0,1.9530635723,2.242456297,-0.34887748$ $54 \backslash \mathrm{C}, 2.6238184497,0.0082933101,1.5701081209 \backslash \mathrm{C}, 2.7251609875,-0.24290512$ $32,-0.9546308212 \backslash C,-0.4374994942,3.2058264073,0.0982930209 \backslash C,-0.369913$ $9107,1.5554042424,2.0126516985 \backslash \mathrm{H}, 3.5376839652,0.4853027562,-0.97149527$ $45 \backslash \mathrm{H}, 3.1500860521,-1.2447819678,-0.8709767487 \backslash \mathrm{H}, 2.1652814946,-0.185344$ $0704,-1.8922573525 \backslash \mathrm{H}, 3.373026992,0.8031478519,1.5210864534 \backslash \mathrm{H}, 2.0055209$ $24,0.1389209592,2.4593405065 \backslash \mathrm{H}, 3.1465149894,-0.9474471179,1.6517314505$ $\backslash \mathrm{H}, 0.3029275422,2.2127267173,2.5724709244 \backslash \mathrm{H},-1.4008668495,1.830078618$, $2.2549687054 \backslash \mathrm{H},-0.2221611136,0.5203409414,2.3237154144 \backslash \mathrm{H}, 0.2600138017$, $3.8663692693,0.6184176809 \backslash \mathrm{H},-0.3328261441,3.3687846419,-0.977451185 \backslash \mathrm{H}$, $-1.4488137681,3.485988066,0.4011170361 \backslash \backslash$ Version=IA32L-G03RevC.02\State $=1-\mathrm{A} \backslash \mathrm{HF}=-750.2258986 \backslash \mathrm{S} 2=0 . \backslash \mathrm{S} 2-1=0 . \backslash \mathrm{S} 2 \mathrm{~A}=0 . \backslash \mathrm{RMSD}=6.486 \mathrm{e}-09 \backslash \mathrm{RMSF}=8.601 \mathrm{e}-0$ $6 \backslash$ Dipole $=0.2355138,0.2096579,0.4275173 \backslash P G=C 01 \quad[\mathrm{X}(\mathrm{C} 16 \mathrm{H} 18 \mathrm{~N} 101)] \backslash \backslash @$

\section{TMAO-}

$1 \backslash 1 \backslash G I N C-A C 27 \backslash F O p t \backslash U B 3 L Y P \backslash 6-31 G(d) \backslash C 16 H 18 N 101$ (1-) \JOH501 \15-May-2006\0

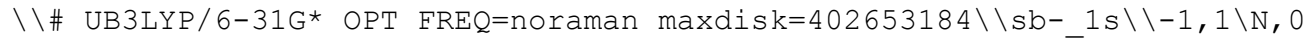
$.0000759714,0.0000881307,0 . \backslash 0,1.399501705,0.000075069,0 . \backslash \bar{C},-2.70807522$ $79,-0.8195172222,0 . \backslash \mathrm{C},-4.1374202227,-1.0102713708,0 . \backslash \mathrm{C},-4.8272104746,-$ $1.1097062119,1.2360387037 \backslash C,-4.8272104746,-1.1097062119,-1.2360387037 \backslash$ $C,-4.1371251432,-1.0340937249,2.4235765945 \backslash C,-4.1371251432,-1.03409372$ $49,-2.4235765945 \backslash C,-2.7355984319,-0.8497200099,2.4223063565 \backslash C,-2.73559$ $84319,-0.8497200099,-2.4223063565 \backslash \mathrm{C},-2.0118614118,-0.7404403465,1.2466$ $207268 \backslash \mathrm{C},-2.0118614118,-0.7404403465,-1.2466207268 \backslash \mathrm{H},-5.9068091153,-1$. $2531155696,1.2241897678 \backslash \mathrm{H},-5.9068091153,-1.2531155696,-1.2241897678 \backslash \mathrm{H}$, $-4.6659658509,-1.1174020539,3.3717457042 \backslash \mathrm{H},-4.6659658509,-1.1174020539$ $,-3.3717457042 \backslash \mathrm{H},-2.213674027,-0.7942551664,3.3728725566 \backslash \mathrm{H},-2.21367402$ $7,-0.7942551664,-3.3728725566 \backslash C,-0.5000768681,-0.5802639716,1.27552243$ $1 \backslash \mathrm{C},-0.5000768681,-0.5802639716,-1.275522431 \backslash \mathrm{C}, 0.1805532238,-1.9328035$ $293,1.6296319888 \backslash C, 0.1805532238,-1.9328035293,-1.6296319888 \backslash C,-0.07178$ $28627,0.4659487467,2.3353034678 \backslash \mathrm{C},-0.0717828627,0.4659487467,-2.335303$ $4678 \backslash \mathrm{H},-0.6362105217,1.3951788628,2.1980777514 \backslash \mathrm{H},-0.6362105217,1.39517$ $88628,-2.1980777514 \backslash \mathrm{H},-0.2051207737,0.1131361561,3.3659821743 \backslash \mathrm{H},-0.205$ $1207737,0.1131361561,-3.3659821743 \backslash \mathrm{H}, 0.9841895227,0.6639042107,2.13503$ $61879 \backslash \mathrm{H}, 0.9841895227,0.6639042107,-2.1350361879 \backslash \mathrm{H},-0.2794604048,-2.777$ $7148462,1.1062084826 \backslash \mathrm{H},-0.2794604048,-2.7777148462,-1.1062084826 \backslash \mathrm{H}, 0.1$ $45235347,-2.1452330736,2.7062297116 \backslash \mathrm{H}, 0.145235347,-2.1452330736,-2.706$ $2297116 \backslash \mathrm{H}, 1.2194416794,-1.8096317758,1.2978288912 \backslash \mathrm{H}, 1.2194416794,-1.80$ $96317758,-1.2978288912 \backslash \backslash$ Version=IA64 L-G03RevD.01 $\backslash$ State $=1-A^{\prime} \backslash \mathrm{HF}=-750.47$ $14771 \backslash \mathrm{S} 2=0 . \backslash \mathrm{S} 2-1=0 . \backslash \mathrm{S} 2 \mathrm{~A}=0 . \backslash \mathrm{RMSD}=8.450 \mathrm{e}-09 \backslash \mathrm{RMSF}=8.439 \mathrm{e}-06 \backslash \mathrm{Thermal}=0 . \backslash \mathrm{Di}$ pole $=-2.8204639,-0.8266547,0 . \backslash \mathrm{PG}=\mathrm{CS} \quad[\mathrm{SG}(\mathrm{C} 2 \mathrm{N1O} 1), \mathrm{X}(\mathrm{C} 14 \mathrm{H} 18)] \backslash \backslash @$

\section{4-carboxy-TMAO•(37)}

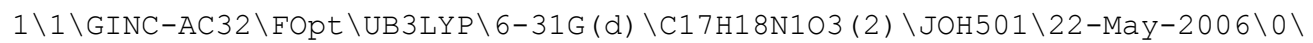

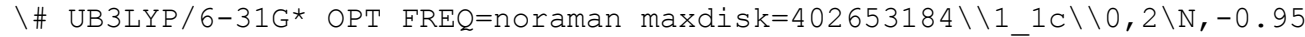
$54030528,-1.7711635486,-0.3333542315 \backslash 0,-1.1928472975,-\overline{3} .0247850926,-0$. $478720141 \backslash \mathrm{C},-0.4425333484,0.9919953844,0.002761959 \backslash \mathrm{C},-0.2220100784,2.4$ $161090091,0.0094781017 \backslash C, 1.098642791,2.9182259338,-0.04920734 \backslash C,-1.315$ 
$8586101,3.3140278379,0.0723804143 \backslash \mathrm{H}, 1.2551503347,3.9934469325,-0.06735$ $62657 \backslash \mathrm{H},-1.1124064082,4.3816494016,0.0806179857 \backslash \mathrm{C}, 2.1597182231,2.05819$ $52289,-0.0820720803 \backslash \mathrm{C},-2.6020575957,2.8386871642,0.1283609598 \backslash \mathrm{H}, 3.1731$ $63514,2.4400765089,-0.1352833181 \backslash \mathrm{H},-3.4447673452,3.5216437453,0.188452$ $9688 \backslash \mathrm{C}, 1.9653752676,0.650735885,-0.0160190808 \backslash \mathrm{C},-2.8312637557,1.449577$ $4279,0.0883877739 \backslash \mathrm{C}, 3.2561651637,-0.1057161147,0.0961239858 \backslash \mathrm{H},-3.85693$ $86473,1.0955911342,0.1106405997 \backslash \mathrm{C}, 0.6817202336,0.0958839557,0.00945659$ $01 \backslash C,-1.7975803956,0.532425442,0.0079303403 \backslash C, 0.4545383323,-1.42231324$ $76,0.0615429801 \backslash C,-2.1629543869,-0.9399440695,-0.0864036559 \backslash C, 0.674767$ $7696,-1.9599730329,1.4985218411 \backslash \mathrm{C},-2.8490973576,-1.4279519028,1.213453$ $9555 \backslash C, 1.3400523153,-2.1802290419,-0.9545275471 \backslash C,-3.0978631255,-1.188$ $6386192,-1.2942770681 \backslash \mathrm{H}, 1.2971677655,-1.7006185509,-1.9377405839 \backslash \mathrm{H},-2$. $6408890684,-0.8134992134,-2.2152821337 \backslash H, 2.3747064609,-2.2320226541,-0$ $.6216365895 \backslash \mathrm{H},-4.0582498091,-0.6859210299,-1.1536639201 \backslash \mathrm{H}, 0.9606502607$ $,-3.1978210137,-1.0464941384 \backslash \mathrm{H},-3.270405481,-2.2611700633,-1.398589501$ $8 \backslash \mathrm{H}, 0.0546861608,-1.4153345319,2.2178793722 \backslash \mathrm{H},-2.234838562,-1.21431253$ $53,2.0928420753 \backslash \mathrm{H}, 0.3945288776,-3.017102911,1.5220012777 \backslash \mathrm{H},-3.01238722$ $76,-2.5066140308,1.1479086911 \backslash \mathrm{H}, 1.7195124366,-1.8465775621,1.788145564$ $4 \backslash \mathrm{H},-3.8143662634,-0.9297676633,1.3461999906 \backslash 0,3.5757853873,-0.8913364$ $662,0.9650322337 \backslash 0,4.1276634251,0.2609361868,-0.8782123208 \backslash \mathrm{H}, 4.9583130$ $975,-0.2131242825,-0.6807497442 \backslash \backslash$ Version=IA64L-G03RevD.01 \State=2-A \HF $=-939.0276368 \backslash \mathrm{S} 2=0.753736 \backslash \mathrm{S} 2-1=0 . \backslash \mathrm{S} 2 \mathrm{~A}=0.75001 \backslash \mathrm{RMSD}=6.704 \mathrm{e}-09 \backslash \mathrm{RMSF}=5.92$ $8 e-06 \backslash$ Thermal $=0 . \backslash$ Dipole $=-0.0737846,1.3834498,-0.0640909 \backslash P G=C 01 \quad[X(C 17 \mathrm{H}$ $18 \mathrm{~N} 103)] \backslash \backslash 0$

\section{4-carboxy-TMAO+}

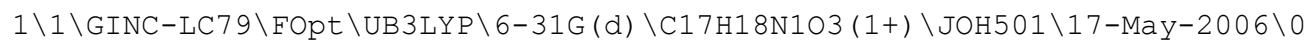
$\backslash \backslash \#$ UB3LYP/6-31G* OPT FREQ=NORAMAN MAXDISK $=671088640 \backslash \backslash 1+1 \backslash \backslash 1,1 \backslash \mathrm{N}, 0.45$ $47451759,-1.8617237678,-0.743453137 \backslash 0,1.1868878253,-2.8008247746,-0.91$ $46916058 \backslash \mathrm{C}, 0.1489007199,0.8747790434,-0.5902224788 \backslash \mathrm{C}, 0.2458102812,2.30$ $37806141,-0.6011236819 \backslash \mathrm{C},-0.0577232524,3.0174566034,0.5805772034 \backslash \mathrm{C}, 0.6$ $300037452,2.9725153233,-1.7920350387 \backslash \mathrm{H},-0.0106383156,4.1023248907,0.57$ $60120746 \backslash \mathrm{H}, 0.7210372766,4.0547456807,-1.775174243 \backslash \mathrm{C},-0.4314102901,2.34$ $23893461,1.7137983969 \backslash \mathrm{C}, 0.8854399559,2.2672771931,-2.9469989609 \backslash \mathrm{H},-0.7$ $075339428,2.8904863923,2.6057057731 \backslash \mathrm{H}, 1.1895364531,2.7824301711,-3.852$ $3863266 \backslash \mathrm{C},-0.4001914026,0.9219027241,1.7784506136 \backslash \mathrm{C}, 0.6916451361,0.870$ $6761691,-2.9755781093 \backslash \mathrm{C},-0.6763485104,0.3578507079,3.1458526499 \backslash \mathrm{H}, 0.81$ $24535389,0.3466751616,-3.9163335936 \backslash \mathrm{C},-0.0619730725,0.1724650577,0.639$ $4547358 \backslash \mathrm{C}, 0.2756722851,0.1931625836,-1.8417855897 \backslash \mathrm{C}, 0.2967322347,-1.32$ $28463507,0.6780231082 \backslash \mathrm{C},-0.2418252376,-1.2436170079,-1.9339544523 \backslash \mathrm{C},-0$ $.7684360881,-2.2932762775,1.2789294727 \backslash C,-1.7739765544,-1.3069807099,-$ $1.742677844 \backslash \mathrm{C}, 1.6704222834,-1.5214920695,1.3604097464 \backslash \mathrm{C}, 0.174416634,-2$ $.0128271099,-3.1854439082 \backslash \mathrm{H}, 2.4454916375,-0.9580713389,0.832656713 \backslash \mathrm{H}, 1$ $.2513173508,-1.9667401184,-3.364331577 \backslash \mathrm{H}, 1.586176665,-1.1574705213,2.3$ $827600183 \backslash \mathrm{H},-0.346851997,-1.5893381046,-4.0468526711 \backslash \mathrm{H}, 1.9443961026,-2$ $.5774318555,1.3831367595 \backslash \mathrm{H},-0.116325376,-3.0628766917,-3.1033635887 \backslash \mathrm{H}$, $-1.7698509443,-2.1099878801,0.8866495031 \backslash \mathrm{H},-2.1151431165,-0.7539426709$ $,-0.8671322261 \backslash \mathrm{H},-0.4767388241,-3.3257406274,1.0690473535 \backslash \mathrm{H},-2.1195806$ $707,-2.3435380149,-1.6846864656 \backslash \mathrm{H},-0.7721889728,-2.1530487602,2.358223$ $044 \backslash \mathrm{H},-2.224116178,-0.8423191682,-2.6248291035 \backslash 0,-0.1361915578,-0.6054$ $948959,3.6539080946 \backslash 0,-1.6037370278,1.0853363707,3.7935072508 \backslash \mathrm{H},-1.693$ $2840397,0.7044811868,4.6892254197 \backslash \backslash$ Version=IA32L-G03RevC.02 \State=1-A $\backslash$ $\mathrm{HF}=-938.7855365 \backslash \mathrm{S} 2=0 . \backslash \mathrm{S} 2-1=0 . \backslash \mathrm{S} 2 \mathrm{~A}=0 . \backslash \mathrm{RMSD}=4.944 \mathrm{e}-09 \backslash \mathrm{RMSF}=7.700 \mathrm{e}-06 \backslash \mathrm{Dip}$ ole $=-0.4305922,0.0020218,-1.1338551 \backslash \mathrm{PG}=\mathrm{C} 01 \quad[\mathrm{X}(\mathrm{C} 17 \mathrm{H} 18 \mathrm{~N} 1 \mathrm{O} 3)] \backslash \backslash \mathrm{e}$

\section{4-carboxy-TMAO-}

$1 \backslash 1 \backslash G I N C-A C 44 \backslash F O p t \backslash U B 3 L Y P \backslash 6-31 G(d) \backslash C 17 H 18 N 103$ (1-) \JOH501 \30-Nov-2006\0

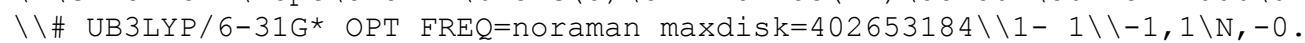
$0388646855,-0.0522342443,-0.000074809 \backslash 0,1.3354625135,-0 . \overline{2} 14393533,0.06$ $27892632 \backslash \mathrm{C},-2.6719352175,0.8911429246,-0.0253584154 \backslash \mathrm{C},-4.1015791429,1$. $1005698975,-0.019871733 \backslash \mathrm{C},-4.7841701202,1.2417495251,-1.2553849363 \backslash \mathrm{C},-$ $4.813195103,1.1660208771,1.1997375484 \backslash \mathrm{H},-5.8890858438,1.330120583,1.16$ $56498347 \backslash \mathrm{C},-4.0886224166,1.2267666304,-2.4288940001 \backslash \mathrm{C},-4.1538690416,1$. 
$0323563291,2.4018893931 \backslash \mathrm{H},-4.6097905155,1.3412510439,-3.3736219947 \backslash \mathrm{H},-$ $4.6970651189,1.0957682115,3.3427209195 \backslash \mathrm{C},-2.6645496424,1.1033742339,-2$ $.4492076887 \backslash \mathrm{C},-2.7638676891,0.7982094746,2.4076710309 \backslash \mathrm{H},-2.2579780856$, $0.6717588173,3.3599629655 \backslash \mathrm{C},-1.9381437343,0.9014811903,-1.2613962623 \backslash \mathrm{C}$ $,-2.0223385972,0.7149965306,1.2407992736 \backslash C,-0.4172927582,0.6840309743$, $-1.2436263344 \backslash \mathrm{C},-0.5400215185,0.4251682994,1.3062535637 \backslash \mathrm{C}, 0.3562152251$ $, 2.0242745311,-1.4019579523 \backslash \mathrm{C}, 0.2414884267,1.6623621283,1.8280537723 \backslash \mathrm{C}$ $, 0.0370719602,-0.3026889477,-2.3554789249 \backslash C,-0.2637649869,-0.765530722$ $7,2.2619856856 \backslash \mathrm{H},-0.6437690178,-1.1609138159,-2.4055067975 \backslash \mathrm{H},-0.901381$ $6761,-1.6158704879,1.9963160289 \backslash \mathrm{H}, 0.1211435667,0.1595513836,-3.3397715$ $005 \backslash \mathrm{H},-0.4253739895,-0.5118058422,3.3166479046 \backslash \mathrm{H}, 1.016625825,-0.655432$ $5943,-2.0238921277 \backslash \mathrm{H}, 0.778964313,-1.0434055909,2.0931408044 \backslash \mathrm{H},-0.08407$ $25119,2.8183385233,-0.7882563163 \backslash \mathrm{H},-0.1028064996,2.5940850245,1.369163$ $4144 \backslash \mathrm{H}, 1.3721402835,1.8166016803,-1.0512192268 \backslash \mathrm{H}, 1.2841202222,1.472875$ $8897,1.5442171729 \backslash \mathrm{H}, 0.3668034867,2.3668719474,-2.4377373935 \backslash \mathrm{H}, 0.156348$ $8041,1.7712415648,2.917014878 \backslash \mathrm{C},-2.0847568323,1.2713182521,-3.80795920$ $96 \backslash \mathrm{H},-5.8648898879,1.3721977054,-1.2505968089 \backslash 0,-1.1663987182,1.985531$ $5146,-4.168734615 \backslash 0,-2.7962566698,0.5689086208,-4.7549853874 \backslash \mathrm{H},-2.3734$ $44606,0.8244914703,-5.5956410194 \backslash \backslash$ Version=IA64L-G03RevD.01 $\backslash$ State $=1-A \backslash H$ $\mathrm{F}=-939.0328511 \backslash \mathrm{S} 2=0 . \backslash \mathrm{S} 2-1=0 . \backslash \mathrm{S} 2 \mathrm{~A}=0 . \backslash \mathrm{RMSD}=8.488 \mathrm{e}-09 \backslash \mathrm{RMSF}=8.122 \mathrm{e}-06 \backslash \mathrm{Ther}$ $\mathrm{mal}=0 . \backslash \mathrm{Dipole}=-2.850086,0.7723049,-0.3966681 \backslash \mathrm{PG}=\mathrm{C} 01 \quad[\mathrm{X}(\mathrm{C} 17 \mathrm{H} 18 \mathrm{~N} 103)] \backslash \backslash @$

\section{5-carboxy-TMAO• (38)}

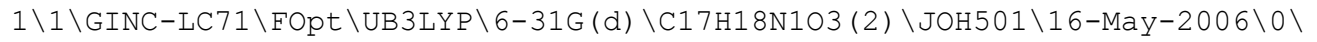
$\backslash \#$ UB3LYP/6-31G* OPT FREQ=NORAMAN MAXDISK=671088640 \2 $1 \backslash \backslash 0,2 \backslash N,-0.041$ $1107755,-2.3888677021,0.586606692 \backslash 0,-0.0063548875,-3.6 \overline{7} 27308265,0.5797$ $860719 \backslash C,-0.0692089973,0.4513731779,0.6070424857 \backslash C,-0.20024643,1.88531$ $18587,0.6171963542 \backslash \mathrm{C},-0.2559377496,2.5794016885,1.8527864732 \backslash \mathrm{C},-0.2611$ $603308,2.5942376971,-0.6057116467 \backslash \mathrm{H},-0.356539676,3.6615442613,1.840739$ $6892 \backslash \mathrm{H},-0.3623512794,3.6742949074,-0.5884095827 \backslash \mathrm{C},-0.1758077338,1.8904$ $766173,3.0383452175 \backslash \mathrm{C},-0.1852131368,1.9193804599,-1.8062112886 \backslash \mathrm{H},-0.21$ $16344173,2.4178955537,3.9873342667 \backslash \mathrm{C},-0.2347950825,2.6153504802,-3.117$ $3497837 \backslash \mathrm{C},-0.0478932226,0.4854753725,3.0293615717 \backslash \mathrm{C},-0.0527314148,0.50$ $95742486,-1.815596075 \backslash \mathrm{H}, 0.0159021732,-0.0317053143,3.9815367268 \backslash \mathrm{H}, 0.00$ $90352413,0.0286527134,-2.7850570116 \backslash C,-0.002059405,-0.2408674208,1.851$ $5199672 \backslash \mathrm{C},-0.0015519186,-0.2256059962,-0.6499593174 \backslash \mathrm{C}, 0.1708443938,-1$. $7371797565,-0.7408327105 \backslash C, 0.1543448626,-1.7549455809,1.9247534926 \backslash C, 1$ $.597797346,-2.0857485514,-1.2316440433 \backslash \mathrm{C}, 1.5684326483,-2.1260630178,2$. $436272564 \backslash \mathrm{C},-0.8766739301,-2.3580867938,-1.6913406147 \backslash \mathrm{C},-0.917624461,-$ $2.3769163577,2.8480906243 \backslash \mathrm{H},-1.8889630621,-2.0804554318,-1.3815127245 \backslash$ $\mathrm{H},-1.9206951615,-2.0810397725,2.5254370475 \backslash \mathrm{H},-0.7211202647,-2.01579930$ $36,-2.7173910226 \backslash \mathrm{H},-0.7760897834,-2.0536073255,3.8826984636 \backslash \mathrm{H},-0.78587$ $28124,-3.4449591584,-1.6636634723 \backslash \mathrm{H},-0.8407571312,-3.4644219249,2.8048$ $983859 \backslash \mathrm{H}, 2.3601004084,-1.6212214152,-0.5980767969 \backslash \mathrm{H}, 2.3461600771,-1.66$ $01959876,1.8230515441 \backslash \mathrm{H}, 1.7304650835,-3.1707843691,-1.2092709714 \backslash \mathrm{H}, 1.6$ $904920667,-3.211861559,2.3998217087 \backslash \mathrm{H}, 1.7449897501,-1.7276778724,-2.25$ $51704668 \backslash \mathrm{H}, 1.7026558385,-1.7856608106,3.4678060638 \backslash 0,-0.1638111952,2.0$ $651173209,-4.198842609 \backslash 0,-0.3699707063,3.9622564567,-3.0038721704 \backslash \mathrm{H},-0$ $.3899999376,4.3009243625,-3.9179286544 \backslash \backslash$ Version=IA32L-G03RevC.02\State $=2-\mathrm{A} \backslash \mathrm{HF}=-939.0442933 \backslash \mathrm{S} 2=0.75375 \backslash \mathrm{S} 2-1=0 . \backslash \mathrm{S} 2 \mathrm{~A}=0.75001 \backslash \mathrm{RMSD}=8.694 \mathrm{e}-09 \backslash \mathrm{RMS}$ $\mathrm{F}=4.189 \mathrm{e}-06 \backslash \mathrm{Dipole}=0.1454676,1.1380775,0.8468046 \backslash \mathrm{PG}=\mathrm{C} 01 \quad[\mathrm{X}(\mathrm{C} 17 \mathrm{H} 18 \mathrm{~N} 103)$ ]$\backslash \backslash @$

\section{5-carboxy-TMAO+}

$1 \backslash 1 \backslash G I N C-L C 73 \backslash F O p t \backslash U B 3 L Y P \backslash 6-31 G(d) \backslash C 17 H 18 N 103(1+) \backslash J O H 501 \backslash 16-M a y-2006 \backslash 0$

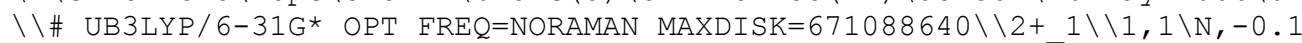
$811845506,-2.3736715401,0.6497401451 \backslash 0,-0.7473798369,-3 . \overline{4} 316646746,0.7$ $435009721 \backslash C,-0.2002109412,0.4151932617,0.6015010643 \backslash C,-0.4280523942,1$. $8282128156,0.5926805445 \backslash \mathrm{C},-0.8790487717,2.4559988446,1.7816907513 \backslash \mathrm{C},-0$ $.2006546261,2.5573275212,-0.60222415 \backslash \mathrm{H},-1.0648652989,3.5261662272,1.77$ $24319255 \backslash \mathrm{H},-0.3524141279,3.6314950767,-0.6103653942 \backslash \mathrm{C},-1.0888530369,1$. $7208789838,2.9305412771 \backslash \mathrm{C}, 0.2159677653,1.9078314251,-1.7474413847 \backslash \mathrm{H},-1$ $.4460742951,2.2056000904,3.8333221516 \backslash C, 0.4950947988,2.6213629296,-3.0$ 
$314361058 \backslash \mathrm{C},-0.7917451491,0.3421037462,2.960517238 \backslash \mathrm{C}, 0.3475831912,0.49$ $84685835,-1.7575489295 \backslash \mathrm{H},-0.8986232213,-0.1931494687,3.8970465743 \backslash \mathrm{H}, 0$. $5983636854,0.0367694166,-2.7072906521 \backslash \mathrm{C},-0.3013414573,-0.2958676949,1$. $8313466006 \backslash \mathrm{C}, 0.111838827,-0.2460334565,-0.6193751074 \backslash \mathrm{C},-0.045846593,-1$ $.7544153679,-0.7313929669 \backslash \mathrm{C}, 0.332025759,-1.6895440229,1.9105690998 \backslash \mathrm{C}, 1$ $.1664367245,-2.4783914044,-1.3860088509 \backslash \mathrm{C}, 1.8745293145,-1.6061478896,1$ $.8247088127 \backslash \mathrm{C},-1.3423044134,-2.1031783086,-1.5013765489 \backslash \mathrm{C},-0.098237000$ $3,-2.5300426782,3.1099832939 \backslash \mathrm{H},-2.2147279476,-1.6620319413,-1.01118509$ $18 \backslash \mathrm{H},-1.1856953533,-2.5948536569,3.2007436645 \backslash \mathrm{H},-1.2634064752,-1.68150$ $34588,-2.5050373435 \backslash \mathrm{H}, 0.3049949727,-2.0753501739,4.0174990125 \backslash \mathrm{H},-1.477$ $2801009,-3.1830332596,-1.581795866 \backslash \mathrm{H}, 0.3048828184,-3.542732534,3.03914$ $38323 \backslash \mathrm{H}, 2.1113536925,-2.246043476,-0.8930140828 \backslash \mathrm{H}, 2.2174428916,-0.9979$ $25549,0.9865316106 \backslash \mathrm{H}, 1.0007622642,-3.5590563363,-1.3791927355 \backslash \mathrm{H}, 2.3209$ $004206,-2.6039935064,1.7683530849 \backslash \mathrm{H}, 1.2301976531,-2.1483119339,-2.4253$ $421385 \backslash \mathrm{H}, 2.2189510381,-1.1260358679,2.7454955911 \backslash 0,0.8335881573,2.0561$ $408455,-4.0490880409 \backslash 0,0.3300535887,3.9544261396,-2.9305667295 \backslash \mathrm{H}, 0.530$ $3419818,4.335928918,-3.806702601 \backslash \backslash$ Version=IA32L-G03RevC .02 $\backslash$ State $=1-A \backslash H$ $\mathrm{F}=-938.795625 \backslash \mathrm{S} 2=0 . \backslash \mathrm{S} 2-1=0 . \backslash \mathrm{S} 2 \mathrm{~A}=0 . \backslash \mathrm{RMSD}=9.212 \mathrm{e}-09 \backslash \mathrm{RMSF}=5.676 \mathrm{e}-06 \backslash \mathrm{Dipol}$ $e=0.0405576,-1.0255334,1.5355877 \backslash P G=C 01 \quad[X(C 17 H 18 N 103)] \backslash \backslash @$

\section{5-carboxy-TMAO-}

$1 \backslash 1 \backslash G I N C-A C 27 \backslash F O p t \backslash U B 3 L Y P \backslash 6-31 G(d) \backslash C 17 H 18 N 103(1-) \backslash J O H 501 \backslash 29-N o v-2006 \backslash 0$ \\\# UB3LYP/6-31G* OPT FREQ=noraman maxdisk $=402653184 \backslash \backslash 2-1 \backslash \backslash-1,1 \backslash \mathrm{N},-0$. $0512728641,0.3166254366,-0.0137244101 \backslash 0,1.3122627306,0.1 \overline{4} 66428607,-0.0$ $195155829 \backslash \mathrm{C},-2.8471832691,-0.0158388674,0.0128896938 \backslash \mathrm{C},-4.2928097851,0$ $.021396771,0.0255124139 \backslash \mathrm{C},-4.9999846145,0.0264354456,-1.2116467046 \backslash \mathrm{C},-$ $4.9805102906,0.0330507191,1.2514514722 \backslash \mathrm{H},-6.0877795257,0.0563515335,-1$ $.1908652804 \backslash \mathrm{H},-6.0664266915,0.0637071624,1.2654150527 \backslash \mathrm{C},-4.3148853991$, $-0.0205797957,-2.3993671198 \backslash \mathrm{C},-4.288171029,-0.0055793289,2.4582966563 \backslash$ $\mathrm{H},-4.8551792862,-0.0263100995,-3.3445109955 \backslash \mathrm{C},-5.0583243134,-0.0029245$ $647,3.7043538235 \backslash C,-2.8996031425,-0.0621436043,-2.4078581487 \backslash C,-2.8656$ $551817,-0.0492733,2.4409843719 \backslash \mathrm{H},-2.3843604565,-0.0997964174,-3.362533$ $1998 \backslash \mathrm{H},-2.3425541063,-0.0807959248,3.3890368755 \backslash \mathrm{C},-2.1599788156,-0.053$ $1142723,-1.2348653163 \backslash C,-2.1484555846,-0.0570225462,1.2628552037 \backslash C,-0$. $6333897022,-0.1373586304,1.2775065872 \backslash C,-0.6425561527,-0.1452592626,-1$ $.2935888064 \backslash C,-0.1743260696,-1.5765521379,1.6351134714 \backslash C,-0.1919800565$ $,-1.5847156139,-1.6795601688 \backslash C,-0.0397550997,0.845257229,2.3160414502 \backslash$ $\mathrm{C},-0.0662697038,0.8370446879,-2.344873693 \backslash \mathrm{H},-0.444878166,1.8505961253$, $2.1572414998 \backslash \mathrm{H},-0.458922812,1.8452647614,-2.1728596396 \backslash \mathrm{H},-0.2400604648$ $, 0.5386869353,3.3491449969 \backslash \mathrm{H},-0.2918604357,0.5353050904,-3.3746965688 \backslash$ $\mathrm{H}, 1.0367165717,0.8691052808,2.1323668034 \backslash \mathrm{H}, 1.0144703064,0.8523808101,-$ $2.1888531187 \backslash \mathrm{H},-0.7493327067,-2.3352493285,1.0941138477 \backslash \mathrm{H},-0.758564140$ $6,-2.348852568,-1.1370559786 \backslash \mathrm{H}, 0.8785611654,-1.6282442812,1.3318496815$ $\backslash \mathrm{H}, 0.8656018641,-1.6500369003,-1.4029852876 \backslash \mathrm{H},-0.2709569023,-1.7833957$ $9,2.7080417551 \backslash \mathrm{H},-0.3094709642,-1.7786563814,-2.7530635267 \backslash 0,-6.279220$ $8933,0.0433314963,3.8075631973 \backslash 0,-4.2814814304,-0.058128862,4.83705042$ $64 \backslash \mathrm{H},-4.9300425822,-0.0464838678,5.5632342662 \backslash \backslash$ Version=IA64L-G03RevD. 0 $1 \backslash \mathrm{State}=1-\mathrm{A} \backslash \mathrm{HF}=-939.0547968 \backslash \mathrm{S} 2=0 . \backslash \mathrm{S} 2-1=0 . \backslash \mathrm{S} 2 \mathrm{~A}=0 . \backslash \mathrm{RMSD}=4.473 \mathrm{e}-09 \backslash \mathrm{RMSF}=5$ $.645 e-06 \backslash$ Thermal=0. $\backslash$ Dipole $=-1.9177578,-0.3822648,0.0929447 \backslash \mathrm{PG}=\mathrm{C} 01 \quad[\mathrm{X}(\mathrm{C}$ $17 \mathrm{H} 18 \mathrm{~N} 103)] \backslash \backslash \mathrm{Q}$

\section{6-carboxy-TMAO•(39)}

$1 \backslash 1 \backslash$ GINC-LC6 $\backslash$ FOpt \UB3LYP \6-31G (d) \C17H18N103 (2) \JOH501\16-May-2006\0 $\backslash \#$ UB3LYP/6-31G* OPT FREQ=NORAMAN MAXDISK $=671088640 \backslash \backslash 3 \_2 \backslash \backslash 0,2 \backslash N, 2.6231$ $585006,0.1888042792,0.2052495098 \backslash 0,3.9066262757,0.2371 \overline{5} 66292,0.1919025$ $963 \backslash C,-0.2325333371,0.0293337783,0.2284055823 \backslash C,-1.6799621535,0.065331$ $461,0.2413415826 \backslash \mathrm{C},-2.3862330731,0.0740067268,-1.0126473455 \backslash \mathrm{C},-2.34833$ $33685,0.0782245381,1.4933644313 \backslash \mathrm{C},-3.8724151905,0.1217652287,-1.099611$ $2626 \backslash \mathrm{H},-3.4279066583,0.108739266,1.5056844844 \backslash \mathrm{C},-1.6666125878,0.032420$ $2267,-2.1950480183 \backslash \mathrm{C},-1.6340768621,0.0456724077,2.6678591392 \backslash \mathrm{H},-2.1952$ $587803,0.0320111258,-3.1400671716 \backslash \mathrm{H},-2.1592909294,0.0488748319,3.61917$ $51602 \backslash \mathrm{C},-0.2660914165,-0.0062858934,-2.1956566191 \backslash \mathrm{C},-0.2286684997,0.01$ $00877235,2.6547030608 \backslash \mathrm{H}, 0.2464152057,-0.0420985052,-3.1508483409 \backslash \mathrm{H}, 0.3$ 
$01100483,-0.018990457,3.601464193 \backslash C, 0.4608294858,0.0009650898,-1.01941$ $74097 \backslash \mathrm{C}, 0.4795100398,0.0105755625,1.4676836759 \backslash \mathrm{C}, 2.0020525827,-0.04894$ $65458,1.5393687974 \backslash \mathrm{C}, 1.9800354671,-0.0647434959,-1.1141253563 \backslash \mathrm{C}, 2.4631$ $738713,-1.4376003269,2.0475236484 \backslash C, 2.4221203405,-1.4649771188,-1.6099$ $383185 \backslash C, 2.5557671653,1.0586258091,2.4646480331 \backslash C, 2.5220973801,1.02594$ $05373,-2.0665064541 \backslash \mathrm{H}, 2.1885691516,2.0405192608,2.1504148518 \backslash \mathrm{H}, 2.16626$ $38126,2.0146310049,-1.7605607752 \backslash \mathrm{H}, 2.2521618915,0.8900203435,3.5008295$ $154 \backslash \mathrm{H}, 2.2013962146,0.8437336717,-3.0952027748 \backslash \mathrm{H}, 3.6456999892,1.0567294$ $455,2.4125639612 \backslash \mathrm{H}, 3.6124380738,1.0184645345,-2.0306882355 \backslash \mathrm{H}, 2.0487141$ $406,-2.2416175607,1.4313170338 \backslash \mathrm{H}, 2.0104811154,-2.25726741,-0.976924597$ $\backslash \mathrm{H}, 3.5547387135,-1.489621794,2.0132903547 \backslash \mathrm{H}, 3.5135755446,-1.5224734641$ $,-1.5859348625 \backslash \mathrm{H}, 2.1294485795,-1.596395165,3.0774452067 \backslash \mathrm{H}, 2.0768110992$ $,-1.6363787242,-2.6341067619 \backslash 0,-4.6700208273,0.1770502051,-0.180846431$ $3 \backslash 0,-4.3192200895,0.0994753341,-2.387413172 \backslash \mathrm{H},-5.2905090839,0.13765804$ $18,-2.3154247557 \backslash \backslash$ Version $=I A 32 \mathrm{~L}-\mathrm{G} 03$ RevC.02 $\backslash$ State $=2-\mathrm{A} \backslash \mathrm{HF}=-939.039068 \backslash \mathrm{S} 2$ $=0.753743 \backslash \mathrm{S} 2-1=0 . \backslash \mathrm{S} 2 \mathrm{~A}=0.75001 \backslash \mathrm{RMSD}=4.827 \mathrm{e}-09 \backslash \mathrm{RMSF}=3.998 \mathrm{e}-06 \backslash \mathrm{Dipole}=-0$. $2949615,-0.268335,-0.3330159 \backslash P G=C 01 \quad[X(C 17 H 18 \mathrm{~N} 103)] \backslash \backslash @$

\section{6-carboxy-TMAO+}

$1 \backslash 1 \backslash G I N C-A C 39 \backslash F O p t \backslash U B 3 L Y P \backslash 6-31 G(d) \backslash C 17 H 18 N 103(1+) \backslash J O H 501 \backslash 23-M a y-2006 \backslash 0$ \\\# UB3LYP/6-31G* OPT FREQ=noraman maxdisk $=1073741824 \backslash \backslash 3+7 \backslash \backslash 1,1 \backslash \mathrm{N}, 0.0$ $090135003,-0.0470079641,-0.0110844044 \backslash 0,1.2121729474,-0.1082276937,0.0$ $108247167 \backslash C,-2.3393134235,1.4707606669,-0.0595104642 \backslash C,-3.4070845192,2$ $.4309592949,-0.0788537778 \backslash \mathrm{C},-4.2949464201,2.4833575137,1.0497150669 \backslash \mathrm{C}$, $-3.5152160538,3.2780583785,-1.2166612929 \backslash \mathrm{C},-5.4585893355,3.4319362944$, $1.1040289315 \backslash \mathrm{H},-4.2998724279,4.0217847953,-1.2341186096 \backslash \mathrm{C},-4.079628830$ $8,1.6391022607,2.1269215709 \backslash \mathrm{C},-2.6308536446,3.1681739699,-2.2714298277$ $\backslash \mathrm{H},-4.7600318877,1.6682845147,2.9686134994 \backslash \mathrm{H},-2.7252527724,3.834445982$ $3,-3.1230661678 \backslash C,-2.9635899201,0.785010103,2.1782548296 \backslash C,-1.64088667$ $47,2.1677820964,-2.2886995448 \backslash \mathrm{H},-1.0158755816,2.0601945764,-3.16755647$ $91 \backslash \mathrm{C},-2.0773044529,0.7203436988,1.1192834171 \backslash \mathrm{C},-1.5331570963,1.2888909$ $537,-1.2242173068 \backslash \mathrm{C},-0.7278224519,0.0448481133,1.3127194278 \backslash \mathrm{C},-0.71733$ $29213,-0.0047911582,-1.3431629727 \backslash \mathrm{C},-0.8089069064,-1.4178738228,1.8349$ $684323 \backslash C,-1.6354267144,-1.2432516933,-1.4538853893 \backslash C, 0.1583857758,0.89$ $23454512,2.2571770759 \backslash \mathrm{C}, 0.3318481306,-0.0110507795,-2.4521330212 \backslash \mathrm{H}, 0.2$ $870069585,1.9044522405,1.8634312226 \backslash \mathrm{H}, 0.9861913899,0.8633091481,-2.409$ $6858512 \backslash \mathrm{H},-0.3419307746,0.9621545553,3.2247561971 \backslash \mathrm{H},-0.1787912107,-0.0$ $242379852,-3.4175157117 \backslash \mathrm{H}, 1.1370335653,0.4310012812,2.3993647663 \backslash \mathrm{H}, 0.9$ $511065643,-0.909015896,-2.3911522862 \backslash \mathrm{H},-1.4503880779,-2.0500102377,1.2$ $19183845 \backslash \mathrm{H},-2.4080385936,-1.2665520265,-0.6842961489 \backslash \mathrm{H}, 0.194578906,-1$. $848986502,1.8849906049 \backslash \mathrm{H},-1.0525396193,-2.1692426824,-1.4250418311 \backslash \mathrm{H},-$ $1.2180314077,-1.3934180395,2.8474442282 \backslash \mathrm{H},-2.1375635368,-1.1818814103$, $-2.4239521002 \backslash \mathrm{H},-2.7913581542,0.2139975457,3.0853335108 \backslash 0,-5.706125674$ $4,4.3036739028,0.2969864496 \backslash 0,-6.234792382,3.2130822333,2.186509539 \backslash \mathrm{H}$, $-6.9557962715,3.87032832,2.1497858559 \backslash \backslash$ Version=IA64L-G03RevD.01 $\backslash$ State $=$ $1-\mathrm{A} \backslash \mathrm{HF}=-938.7903753 \backslash \mathrm{S} 2=0 . \backslash \mathrm{S} 2-1=0 . \backslash \mathrm{S} 2 \mathrm{~A}=0 . \backslash \mathrm{RMSD}=4.589 \mathrm{e}-09 \backslash \mathrm{RMSF}=2.417 \mathrm{e}-06$ $\backslash$ Thermal $=0 . \backslash$ Dipole $=1.2859028,-1.6345276,0.0628427 \backslash \mathrm{PG}=\mathrm{C} 01 \quad[\mathrm{X}(\mathrm{C} 17 \mathrm{H} 18 \mathrm{~N} 103$ )$] \backslash \backslash 0$

\section{6-carboxy-TMAO-}

$1 \backslash 1 \backslash$ GINC-AC5 4 \FOpt \UB3LYP \6-31G(d) \C17H18N1O3 (1-) \JOH501 \29-Nov-2006\0

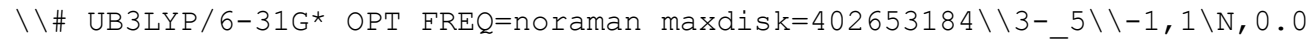
$813031913,0.0860330546,0.1117351131 \backslash 0,1.0431831038,-0.9051281016,0.159$ $9529446 \backslash \mathrm{C},-2.4067149201,1.4841558158,-0.0335669408 \backslash \mathrm{C},-3.5431466213,2.3$ $803366464,-0.0929176008 \backslash \mathrm{C},-4.2187072473,2.7335280552,1.1318926376 \backslash \mathrm{C},-3$ $.9502180228,2.8691727186,-1.3620970467 \backslash \mathrm{C},-5.3744311419,3.6363434234,1$. $1690423503 \backslash \mathrm{H},-4.7975632827,3.5377681183,-1.4143522566 \backslash \mathrm{C},-3.7572741649$, $2.1932841808,2.3380946965 \backslash \mathrm{C},-3.2783661658,2.4898481126,-2.5036161851 \backslash \mathrm{H}$ $,-4.2650868987,2.4519626546,3.2596657302 \backslash \mathrm{H},-3.6076768094,2.8698750261$, $-3.469663808 \backslash C,-2.6632339625,1.3293329475,2.3782024965 \backslash C,-2.1728904481$ $, 1.6247696788,-2.4424993651 \backslash \mathrm{H},-1.6665683075,1.3475609195,-3.3617034766$ $\backslash \mathrm{C},-1.9825404736,0.9525367169,1.2273454323 \backslash \mathrm{C},-1.7163807918,1.122887338$ $8,-1.2330413851 \backslash \mathrm{C},-0.8132892181,-0.001790359,1.2988127652 \backslash \mathrm{C},-0.5427212$ 
$644,0.1448539859,-1.2332547692 \backslash C,-1.3415351988,-1.4482036874,1.4885718$ $029 \backslash \mathrm{C},-0.9975324797,-1.2488110699,-1.7625385072 \backslash \mathrm{C}, 0.1200751856,0.35004$ $29703,2.4833265764 \backslash \mathrm{C}, 0.6061623272,0.6445275224,-2.1458711896 \backslash \mathrm{H}, 0.39217$ $13199,1.4106675404,2.4492191049 \backslash \mathrm{H}, 0.875058594,1.673603058,-1.883385672$ $6 \backslash \mathrm{H},-0.3260326122,0.1262603139,3.4595306224 \backslash \mathrm{H}, 0.355767426,0.6003436812$ $,-3.2126828472 \backslash \mathrm{H}, 1.0213181,-0.2494472485,2.3336141639 \backslash \mathrm{H}, 1.4609934807,-$ $0.0031581496,-1.9388942082 \backslash \mathrm{H},-2.1935694113,-1.6672130682,0.8377532236 \backslash$ $\mathrm{H},-1.9600635783,-1.5551476114,-1.3398170456 \backslash \mathrm{H},-0.4876698424,-2.0835431$ $541,1.2121791288 \backslash \mathrm{H},-0.2209202083,-1.9551510461,-1.4534977555 \backslash \mathrm{H},-1.6499$ $433292,-1.6389718555,2.5241033216 \backslash \mathrm{H},-1.0967709499,-1.254835881,-2.8553$ $17945 \backslash \mathrm{H},-2.3441218667,0.9359586541,3.3382077938 \backslash 0,-5.9329352003,4.2173$ $646338,0.2431054518 \backslash 0,-5.8710456776,3.831177476,2.4432071238 \backslash \mathrm{H},-6.6161$ $926329,4.4399359882,2.2954255245 \backslash \backslash$ Version=IA64L-G03RevD.01 \State=1-A $\backslash H$ $\mathrm{F}=-939.0488007 \backslash \mathrm{S} 2=0 . \backslash \mathrm{S} 2-1=0 . \backslash \mathrm{S} 2 \mathrm{~A}=0 . \backslash \mathrm{RMSD}=3.687 \mathrm{e}-09 \backslash \mathrm{RMSF}=1.210 \mathrm{e}-05 \backslash \mathrm{Ther}$ $\mathrm{mal}=0 . \backslash \mathrm{Dipole}=-2.1684548,1.1171189,0.3009595 \backslash \mathrm{PG}=\mathrm{C} 01 \quad[\mathrm{X}(\mathrm{C} 17 \mathrm{H} 18 \mathrm{~N} 103)] \backslash \backslash @$

\section{4,5-dicarboxy-TMAO• (40)}

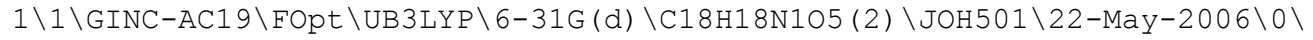

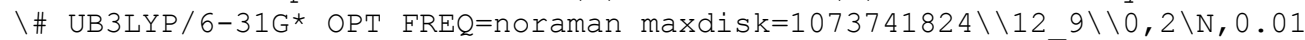
$59898863,-0.0156131994,0.0097144069 \backslash 0,1.299528387,-0.0400064827,0.0299$ $01689 \backslash C,-2.8149794838,0.0243928866,0.0370533538 \backslash C,-4.2468596853,-0.020$ $6093803,-0.079123274 \backslash C,-4.9582116601,1.1609121803,-0.3684085662 \backslash C,-4.9$ $546102411,-1.2338209802,0.1085219224 \backslash \mathrm{H},-6.0381814092,1.1196874225,-0.4$ $522065115 \backslash \mathrm{H},-6.0372104871,-1.225019997,0.0152237949 \backslash \mathrm{C},-4.3060309496,2$. $3611968256,-0.5236603659 \backslash \mathrm{C},-4.2769071338,-2.3885070142,0.4095516646 \backslash \mathrm{C}$, $-5.1098813461,3.5959700703,-0.7455863632 \backslash \mathrm{H},-4.8091028835,-3.3223716025$ $, 0.5654618636 \backslash \mathrm{C},-2.8808015684,2.4263254012,-0.3894484257 \backslash \mathrm{C},-2.87235064$ $12,-2.360579572,0.5029282467 \backslash \mathrm{C},-2.2612226748,3.7967609267,-0.548805850$ $2 \backslash \mathrm{H},-2.3535106757,-3.2874416743,0.7252772416 \backslash \mathrm{C},-2.1317074839,1.2823051$ $122,-0.1156383853 \backslash C,-2.1367121473,-1.2020881716,0.3103944884 \backslash C,-0.6101$ $966295,1.3513725574,0.1090060103 \backslash C,-0.6233122178,-1.3032656745,0.38927$ $19787 \backslash C,-0.3167547875,1.8933372791,1.5317540249 \backslash C,-0.167862658,-1.6837$ $854116,1.8200212941 \backslash \mathrm{C}, 0.1285249372,2.1964810399,-0.9552609367 \backslash \mathrm{C},-0.096$ $9192171,-2.3485902185,-0.6232054824 \backslash \mathrm{H},-0.2595130932,1.9984638536,-1.95$ $85201976 \backslash \mathrm{H},-0.4462478243,-2.1131276801,-1.6333446059 \backslash \mathrm{H}, 0.0497395024,3$. $2604141763,-0.741686788 \backslash \mathrm{H},-0.4412041167,-3.3527982436,-0.3622903895 \backslash \mathrm{H}$, $1.1833250893,1.9248494642,-0.9234090013 \backslash \mathrm{H}, 0.9943166589,-2.3346569955,-$ $0.6160296898 \backslash \mathrm{H},-0.8245050875,1.2921115128,2.2928032383 \backslash \mathrm{H},-0.5748588818$ $,-0.9930977914,2.5642386078 \backslash \mathrm{H}, 0.7617687056,1.8488622926,1.7094643752 \backslash \mathrm{H}$ $, 0.923288858,-1.6526246599,1.8664996108 \backslash \mathrm{H},-0.6636107461,2.9241833959,1$ $.6208430317 \backslash \mathrm{H},-0.5059639514,-2.6930764995,2.0740931971 \backslash 0,-1.8213673482$ $, 4.4795734536,0.3485539731 \backslash 0,-2.2320550382,4.1698284602,-1.8470144374 \backslash$ $\mathrm{H},-1.9137452849,5.0930543886,-1.8550283303 \backslash 0,-4.7088807431,4.735798802$ $6,-0.6246235403 \backslash 0,-6.3968087587,3.329484875,-1.0886827551 \backslash \mathrm{H},-6.8284251$ $694,4.2000548712,-1.1698141181 \backslash \backslash$ Version=IA64L-G03RevD.01 \State=2-A \HF= $-1127.5930955 \backslash \mathrm{S} 2=0.753735 \backslash \mathrm{S} 2-1=0 . \backslash \mathrm{S} 2 \mathrm{~A}=0.75001 \backslash \mathrm{RMSD}=8.046 \mathrm{e}-09 \backslash \mathrm{RMSF}=3.37$ $6 \mathrm{e}-06 \backslash$ Thermal $=0 . \backslash$ Dipole $=-1.388591,-1.0664564,-0.2187582 \backslash \mathrm{PG}=\mathrm{C} 01 \quad[\mathrm{X}(\mathrm{C} 18 \mathrm{H}$ $18 \mathrm{~N} 105)] \backslash \backslash 0$

\section{4,5-dicarboxy-TMAO+}

$1 \backslash 1 \backslash G I N C-A C 48 \backslash F O p t \backslash U B 3 L Y P \backslash 6-31 G(d) \backslash C 18 H 18 N 105(1+) \backslash J O H 501 \backslash 22-M a y-2006 \backslash 0$

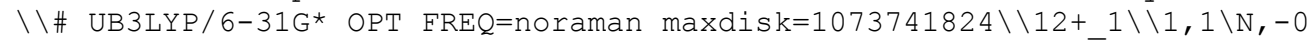
$.0186849661,0.0099146713,0.0392304477 \backslash 0,1.1840774069,0.011 \overline{9} 695439,0.05$ $80444646 \backslash C,-2.389062003,1.4328855988,-0.0123268411 \backslash C,-3.4579187728,2.3$ $793667371,-0.0538639628 \backslash \mathrm{C},-4.3575377394,2.4378085349,1.0339030451 \backslash \mathrm{C},-3$ $.5970842117,3.2277064735,-1.1816716759 \backslash \mathrm{H},-5.1800955841,3.1437862884,1$. $0098248259 \backslash \mathrm{H},-4.4026862104,3.9561219407,-1.1927026828 \backslash \mathrm{C},-4.2150995684$, $1.5952247649,2.1159562276 \backslash \mathrm{C},-2.7247301741,3.1314385552,-2.2449919349 \backslash \mathrm{C}$ $,-5.1865299919,1.6606349332,3.2526553076 \backslash \mathrm{H},-2.8278672649,3.7877754853$, $-3.1028975349 \backslash C,-3.0923593844,0.715731749,2.2053997449 \backslash C,-1.726169862$, $2.1361424563,-2.2514290387 \backslash \mathrm{C},-2.9402619456,-0.131868208,3.4534016217 \backslash \mathrm{H}$ $,-1.1053247004,2.0266888515,-3.1331115873 \backslash \mathrm{C},-2.1502542233,0.679534735$, $1.1786947434 \backslash C,-1.5911405845,1.263871321,-1.1820791243 \backslash C,-0.7583519762$ 
$, 0.0634768406,1.3731199855 \backslash C,-0.7396946318,-0.0038838403,-1.2919989345$ $\backslash C,-0.6991109408,-1.4134245283,1.8731157703 \backslash C,-1.6180290951,-1.2732739$ $837,-1.3768810808 \backslash \mathrm{C}, 0.0886784477,0.9752490315,2.2958489648 \backslash \mathrm{C}, 0.3053661$ $281,0.0054837381,-2.4042717786 \backslash \mathrm{H}, 0.1730362988,1.9795605269,1.870612255$ $\backslash \mathrm{H}, 0.9328353094,0.8998060755,-2.3789232715 \backslash \mathrm{H},-0.4200815068,1.037200772$ $1,3.2586558747 \backslash \mathrm{H},-0.2086616454,-0.0400287946,-3.367088091 \backslash \mathrm{H}, 1.08693871$ $92,0.559439773,2.4429668727 \backslash \mathrm{H}, 0.9521464182,-0.8719864365,-2.3306390754$ $\backslash \mathrm{H},-1.413149097,-2.0599390467,1.3611016306 \backslash \mathrm{H},-2.380737594,-1.313710973$ $4,-0.598321965 \backslash \mathrm{H}, 0.3142293456,-1.8006667624,1.7388266639 \backslash \mathrm{H},-1.00275858$ $9,-2.1775221788,-1.3404765134 \backslash \mathrm{H},-0.9114287802,-1.4174246054,2.94153344$ $18 \backslash \mathrm{H},-2.1304901839,-1.2436901231,-2.3429826476 \backslash 0,-2.1683344402,0.11154$ $28257,4.3530421022 \backslash 0,-3.68994472,-1.2417354936,3.3671668139 \backslash \mathrm{H},-3.63328$ $63749,-1.7011829234,4.228838914 \backslash 0,-5.052360944,1.058600848,4.296480982$ $1 \backslash 0,-6.2275369244,2.474207715,2.9945188765 \backslash \mathrm{H},-6.7945434432,2.474107111$ $5,3.7894481638 \backslash \backslash$ Version $=I A 64 \mathrm{~L}-\mathrm{G} 03$ RevD. $01 \backslash$ State $=1-\mathrm{A} \backslash \mathrm{HF}=-1127.3478435 \backslash \mathrm{S} 2$ $=0 . \backslash S 2-1=0 . \backslash S 2 A=0 . \backslash R M S D=8.828 e-09 \backslash R M S F=6.124 e-06 \backslash$ Thermal=0.$\backslash$ Dipole $=0.6$ $620578,-0.3246105,-2.3876359 \backslash P G=C 01 \quad[X(C 18 H 18 N 105)] \backslash \backslash @$

\section{4,5-dicarboxy-TMAO-}

$1 \backslash 1 \backslash G I N C-A C 47 \backslash F O p t \backslash U B 3 L Y P \backslash 6-31 G(d) \backslash C 18 H 18 N 105$ (1-) \JOH501\30-Nov-2006\0

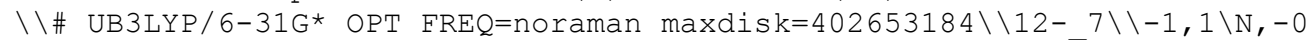
$.0312589342,-0.1858757895,0.1103377298 \backslash 0,1.3213670761,0.0 \overline{0} 25983198,0.0$ $527361062 \backslash \mathrm{C},-2.8229253797,0.0148509015,0.024707905 \backslash \mathrm{C},-4.2628665306,-0$. $0754925335,0.0928609368 \backslash \mathrm{C},-4.9865964583,-0.5138426859,-1.0230067514 \backslash \mathrm{C}$, $-4.9496425147,0.3090721138,1.2803487642 \backslash \mathrm{H},-6.0681079113,-0.5678734359$, $-0.9615523353 \backslash \mathrm{H},-6.0347704,0.229503691,1.3011399596 \backslash \mathrm{C},-4.3521068076,-0$ $.844963199,-2.2144261595 \backslash C_{,}-4.2505307011,0.7860182605,2.3582282803 \backslash C_{r}-$ $5.1594224893,-1.2078890541,-3.3829245769 \backslash \mathrm{H},-4.7716633124,1.0944454162$, $3.2627098018 \backslash \mathrm{C},-2.9170832335,-0.7525243859,-2.292826344 \backslash \mathrm{C},-2.841677000$ $8,0.8741190103,2.2985939573 \backslash C,-2.3020123761,-1.1407067952,-3.613002427$ $6 \backslash \mathrm{H},-2.3046224665,1.2490519449,3.1635111975 \backslash \mathrm{C},-2.1537415216,-0.3309325$ $074,-1.2074596093 \backslash \mathrm{C},-2.1247925983,0.4891177302,1.1733977478 \backslash \mathrm{C},-0.63157$ $51702,-0.1865208935,-1.2625376905 \backslash \mathrm{C},-0.616081577,0.6310126437,1.189785$ $282 \backslash C,-0.2481159348,1.1082999049,-2.0286304953 \backslash C,-0.1953627343,2.12963$ $1508,1.1209462098 \backslash \mathrm{C}, 0.0625491278,-1.4050133296,-1.9269900871 \backslash \mathrm{C},-0.0181$ $407196,0.0254347548,2.4879985617 \backslash \mathrm{H},-0.3841706242,-2.3445415473,-1.5859$ $115948 \backslash \mathrm{H},-0.3924235525,-0.9936450826,2.634370156 \backslash \mathrm{H}, 0.0393845763,-1.366$ $7740098,-3.016943276 \backslash \mathrm{H},-0.2474922703,0.6225572877,3.3780865034 \backslash \mathrm{H}, 1.098$ $6601749,-1.3553960209,-1.5839702363 \backslash \mathrm{H}, 1.0625728208,-0.0179095994,2.339$ $0373556 \backslash \mathrm{H},-0.8505192742,1.961315752,-1.6988913577 \backslash \mathrm{H},-0.7725619464,2.68$ $20570851,0.3729502471 \backslash \mathrm{H}, 0.8045010373,1.292339921,-1.7846895325 \backslash \mathrm{H}, 0.862$ $2376389,2.134327671,0.8394978286 \backslash \mathrm{H},-0.3840667114,0.9969382947,-3.10676$ $92918 \backslash \mathrm{H},-0.3245863364,2.6340691664,2.0863847065 \backslash 0,-1.8548083874,-0.387$ $9621203,-4.4516890567 \backslash 0,-2.2927883356,-2.4891359248,-3.7814094078 \backslash \mathrm{H},-1$ $.9679600923,-2.6223934664,-4.6915492808 \backslash 0,-4.7777072947,-1.357033188,-$ $4.5369933305 \backslash 0,-6.4969548915,-1.3737351433,-3.0943898431 \backslash \mathrm{H},-6.88873596$ $33,-1.5792506653,-3.9615765521 \backslash \backslash$ Version=IA64L-G03RevD.01 $\backslash$ State $=1-A \backslash H F=$ $-1127.6074561 \backslash \mathrm{S} 2=0 . \backslash \mathrm{S} 2-1=0 . \backslash \mathrm{S} 2 \mathrm{~A}=0 . \backslash \mathrm{RMSD}=3.311 \mathrm{e}-09 \backslash \mathrm{RMSF}=1.261 \mathrm{e}-05 \backslash \mathrm{Therm}$ $\mathrm{al}=0 . \backslash \mathrm{Dipole}=-2.7129563,-0.2363472,0.10605 \backslash \mathrm{PG}=\mathrm{C} 01 \quad[\mathrm{X}(\mathrm{C} 18 \mathrm{H} 18 \mathrm{N1O5})] \backslash \backslash @$

\section{4,6-dicarboxy-TMAO• (41)}

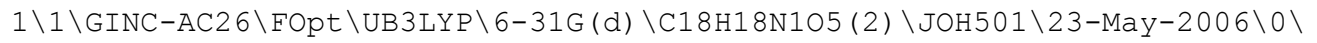
$\backslash \#$ UB3LYP/6-31G* OPT FREQ=noraman maxdisk=671088640\\13 $3 \backslash \backslash 0,2 \backslash N, 0.039$ $7096056,0.0067442308,0.058186478 \backslash 0,1.3228599056,-0.0313 \overline{4} 95777,0.079346$ $5418 \backslash \mathrm{C},-2.8060753738,0.0947448349,0.0199803838 \backslash \mathrm{C},-4.2500310799,-0.0026$ $938616,0.1026114214 \backslash \mathrm{C},-4.966521769,-0.5808146738,-0.998419743 \backslash \mathrm{C},-4.916$ $7999274,0.4770132247,1.2581504704 \backslash C,-6.4441681571,-0.787163737,-0.9935$ $152761 \backslash \mathrm{H},-5.9931785729,0.4031826804,1.3024778611 \backslash \mathrm{C},-4.2656956981,-0.97$ $23702432,-2.1172858555 \backslash \mathrm{C},-4.2058278729,1.0255154061,2.2980366076 \backslash \mathrm{H},-4$. $7270350016,1.4034189818,3.1732771062 \backslash \mathrm{C},-2.8703863242,-0.7939528326,-2$. $2409213945 \backslash \mathrm{C},-2.8044609279,1.0801847886,2.2463311546 \backslash \mathrm{C},-2.3665079689,-$ $1.1262645448,-3.6160739074 \backslash \mathrm{H},-2.2676746089,1.4913279323,3.0949125471 \backslash \mathrm{C}$ $,-2.1141522763,-0.2892064362,-1.1800816726 \backslash C,-2.0987785453,0.611987171$ 
$9,1.1531487719 \backslash C,-0.5926658735,-0.1218642095,-1.2969182713 \backslash C,-0.580855$ $4034,0.6582883415,1.2383077911 \backslash C,-0.2500589882,1.1630033349,-2.0954435$ $383 \backslash \mathrm{C},-0.0682396222,2.1170837714,1.3316033262 \backslash \mathrm{C}, 0.0843747678,-1.360689$ $6251,-1.92944718 \backslash \mathrm{C},-0.0893153996,-0.1452881283,2.467120971 \backslash \mathrm{H},-0.299502$ $5812,-2.2839644923,-1.4839220401 \backslash \mathrm{H},-0.4762500136,-1.1684951001,2.43516$ $11242 \backslash \mathrm{H},-0.0611343455,-1.3899209878,-3.0072558979 \backslash \mathrm{H},-0.4219003136,0.32$ $23506815,3.3972872561 \backslash \mathrm{H}, 1.1543883327,-1.2978281594,-1.7329998619 \backslash \mathrm{H}, 1.0$ $015048483,-0.1808922857,2.4576490423 \backslash \mathrm{H},-0.7338453745,2.0393432861,-1.6$ $526838306 \backslash \mathrm{H},-0.453199922,2.7294414748,0.5112705518 \backslash \mathrm{H}, 0.8339270552,1.30$ $79309601,-2.0683615117 \backslash \mathrm{H}, 1.0237669206,2.1136539301,1.2903572764 \backslash \mathrm{H},-0.5$ $850309274,1.0674879545,-3.1285016628 \backslash \mathrm{H},-0.3857547751,2.5752015252,2.27$ $29971776 \backslash 0,-1.7690234135,-0.3880257821,-4.3717440699 \backslash 0,-2.7475775028,-$ $2.3719295128,-3.9914649544 \backslash \mathrm{H},-2.4606049518,-2.4710859722,-4.9197892284$ $\backslash \mathrm{H},-4.8010265251,-1.4149976385,-2.9460764776 \backslash 0,-7.2239214756,-0.538832$ $6967,-0.092489025 \backslash 0,-6.8967142838,-1.319714567,-2.1617787996 \backslash \mathrm{H},-7.8589$ $656391,-1.4164594473,-2.0395496619 \backslash \backslash$ Version=IA64L-G03RevD.01 \State=2-A $\backslash \mathrm{HF}=-1127.5945562 \backslash \mathrm{S} 2=0.753726 \backslash \mathrm{S} 2-1=0 . \backslash \mathrm{S} 2 \mathrm{~A}=0.75001 \backslash \mathrm{RMSD}=9.422 \mathrm{e}-09 \backslash \mathrm{RMSF}=$ $1.920 e-06 \backslash$ Thermal $=0 . \backslash$ Dipole $=-0.7000625,-0.1454182,-0.0525496 \backslash P G=C 01 \quad[X$ (C18H18N105) ] \\@

\section{4,6-dicarboxy-TMAO+}

$1 \backslash 1 \backslash$ GINC-LC151 \FOpt \UB3LYP \6-31G (d) \C18H18N1O5 (1+) \JOH501\25-May-2006

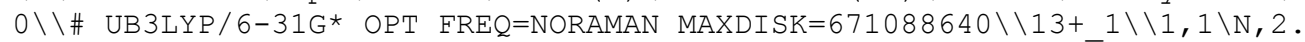
$3515905119,-1.1062842878,-0.6623608543 \backslash 0,3.5546737872,-1.0979698637,-0$ $.6695372411 \backslash \mathrm{C}, 0.0059911447,0.3628677222,-0.6649087628 \backslash \mathrm{C},-1.0412699239$, $1.3467737667,-0.7116407652 \backslash \mathrm{C},-1.9712585158,1.3932172409,0.375443183 \backslash \mathrm{C}$, $-1.1103938334,2.2150545522,-1.83654614 \backslash \mathrm{C},-3.1227533392,2.3592668489,0$. $4145201206 \backslash \mathrm{H},-1.8783453358,2.9759472895,-1.8507294183 \backslash \mathrm{C},-1.8181656217$, $0.5111796283,1.4236517166 \backslash \mathrm{C},-0.2200981961,2.0966336499,-2.8817799422 \backslash \mathrm{H}$ $,-2.5483319103,0.5125487685,2.2205371503 \backslash \mathrm{H},-0.2831139158,2.7755112247$, $-3.7262563467 \backslash C,-0.6985552656,-0.3485682693,1.5466963857 \backslash C, 0.731924459$ $8,1.0612319254,-2.8965379941 \backslash \mathrm{C},-0.6235085762,-1.080072149,2.8603158392$ $\backslash \mathrm{H}, 1.3567686163,0.9326841188,-3.7722912002 \backslash \mathrm{C}, 0.2561368563,-0.397506022$ $4,0.5231263671 \backslash \mathrm{C}, 0.8061590831,0.1738185277,-1.837596936 \backslash \mathrm{C}, 1.6379221145$ $,-1.0525262352,0.6856592744 \backslash C, 1.6003096033,-1.129470818,-1.9698047423 \backslash$ $C, 1.6692208246,-2.5473472191,1.1337724451 \backslash C, 0.6716223796,-2.3616811624$ $,-2.036138215 \backslash \mathrm{C}, 2.5253916129,-0.1824601977,1.606406587 \backslash \mathrm{C}, 2.6177951735$, $-1.165661279,-3.107730617 \backslash \mathrm{H}, 2.6221179694,0.8295096284,1.2024588128 \backslash \mathrm{H}, 3$ $.2799533969,-0.2967727155,-3.1054303249 \backslash \mathrm{H}, 2.0483872862,-0.1424509533,2$ $.5842409141 \backslash \mathrm{H}, 2.079939256,-1.2002498975,-4.0577582966 \backslash \mathrm{H}, 3.5175379425,-$ $0.6231951812,1.7158001345 \backslash \mathrm{H}, 3.2332273551,-2.0659893032,-3.0391763966 \backslash \mathrm{H}$ $, 0.9671095088,-3.1670264404,0.5739915974 \backslash \mathrm{H},-0.0824270976,-2.3698563149$ $,-1.248648146 \backslash \mathrm{H}, 2.6816945386,-2.940156445,1.0089941524 \backslash \mathrm{H}, 1.2497409957$, $-3.2902226644,-2.005667478 \backslash \mathrm{H}, 1.4193307506,-2.5844922435,2.1922607382 \backslash \mathrm{H}$ $, 0.1476483939,-2.3137563234,-2.9952249838 \backslash 0,0.3810133891,-1.2874833656$ $, 3.5119576171 \backslash 0,-1.8442888184,-1.4628597094,3.2717869745 \backslash \mathrm{H},-1.73775895$ $38,-1.871670912,4.1533379095 \backslash 0,-3.3264551987,3.2556875485,-0.377111729$ $9 \backslash 0,-3.9380188756,2.1253505645,1.4634848333 \backslash \mathrm{H},-4.6488265311,2.79332392$ $36,1.4199967066 \backslash \backslash$ Version=IA32L-G03RevC.02 $\backslash$ State $=1-A \backslash H F=-1127.3494342 \backslash \mathrm{S}$ $2=0 . \backslash S 2-1=0 . \backslash S 2 A=0 . \backslash R M S D=7.074 e-09 \backslash R M S F=5.139 e-06 \backslash D i p o l e=0.8308629,-1$. $2648987,-0.9127072 \backslash P G=C 01 \quad[X(C 18 H 18 N 105)] \backslash \backslash @$

\section{4,6-dicarboxy-TMAO-}

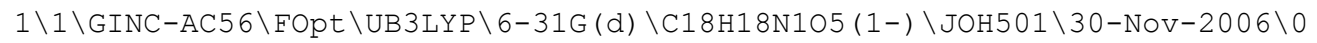

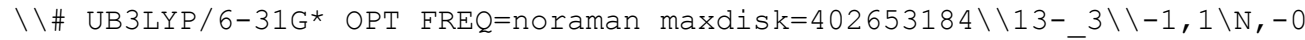
$.0417945215,0.1817765242,-0.0786725394 \backslash 0,1.3139668442,0.1 \overline{0} 66438499,-0$. $1162930748 \backslash \mathrm{C},-2.8310543509,-0.0023485051,-0.0226211604 \backslash \mathrm{C},-4.2695114989$ $, 0.1726007032,-0.1069894414 \backslash C,-4.9574412501,0.7676432406,1.0215532879 \backslash$ C, $-4.9518305837,-0.2406015889,-1.2693506869 \backslash \mathrm{C},-6.3906016622,1.07949059$ $36,1.0218981571 \backslash \mathrm{H},-6.0245328811,-0.1142547069,-1.3081809807 \backslash \mathrm{C},-4.22684$ $71711,1.0439083472,2.1637685558 \backslash \mathrm{C},-4.2671222694,-0.7937118854,-2.33789$ $71426 \backslash \mathrm{H},-4.811338173,-1.124895284,-3.2199691142 \backslash \mathrm{C},-2.8576278546,0.7406$ $889979,2.2953628079 \backslash \mathrm{C},-2.8718769738,-0.8967479657,-2.2952693723 \backslash \mathrm{C},-2.3$ 
$713517225,0.8619504335,3.6942434197 \backslash \mathrm{H},-2.3399941198,-1.2877154978,-3.1$ $581685819 \backslash \mathrm{C},-2.1092353216,0.2692894604,1.1885533986 \backslash \mathrm{C},-2.1500960265,-0$ $.4937063583,-1.1819996744 \backslash C,-0.5932978227,0.0084244866,1.2869990915 \backslash C$, $-0.639470811,-0.5138156213,-1.2300416728 \backslash C,-0.2690153794,-1.383028394$, $1.9084133918 \backslash C,-0.0982300366,-1.9643849648,-1.3484265709 \backslash C, 0.136929921$ $2,1.105448227,2.1100282992 \backslash \mathrm{C},-0.157374227,0.3008464147,-2.4603660632 \backslash \mathrm{H}$ $,-0.2648691653,2.0972331359,1.870913473 \backslash \mathrm{H},-0.6113137285,1.2972154085,-$ $2.4476087626 \backslash \mathrm{H}, 0.0799868233,0.9352207672,3.1843033751 \backslash \mathrm{H},-0.4076040198$, $-0.1912854521,-3.4065693199 \backslash \mathrm{H}, 1.1780294668,1.069399371,1.7861789612 \backslash \mathrm{H}$, $0.92514895,0.4072025177,-2.3625467634 \backslash \mathrm{H},-0.8621595219,-2.1749086295,1$. $438222338 \backslash \mathrm{H},-0.5872123362,-2.6431173393,-0.6441722455 \backslash \mathrm{H}, 0.7913868148,-$ $1.5848146602,1.7320007299 \backslash \mathrm{H}, 0.9707722967,-1.9039046527,-1.1159371875 \backslash \mathrm{H}$ $,-0.4793433233,-1.3944531893,2.9795340002 \backslash \mathrm{H},-0.2421642554,-2.366376051$ $2,-2.3588722651 \backslash 0,-1.7402294667,0.0507305004,4.3503919895 \backslash 0,-2.8047956$ $245,2.0191246488,4.2948127686 \backslash \mathrm{H},-2.5119411412,1.9258359165,5.220212208$ $3 \backslash \mathrm{H},-4.7371435382,1.4725149346,3.0155371975 \backslash 0,-7.211997377,0.908275252$ $2,0.1263535979 \backslash 0,-6.8294483593,1.6414822761,2.203790547 \backslash \mathrm{H},-7.777224602$ $7,1.7886847389,2.0379410242 \backslash \backslash$ Version=IA64L-G03RevD.01 \State=1-A \HF=-11 $27.609279 \backslash \mathrm{S} 2=0 . \backslash \mathrm{S} 2-1=0 . \backslash \mathrm{S} 2 \mathrm{~A}=0 . \backslash \mathrm{RMSD}=2.122 \mathrm{e}-09 \backslash \mathrm{RMSF}=7.267 \mathrm{e}-06 \backslash \mathrm{Therma} I=0$ .$\backslash$ Dipole $=-2.0182371,0.1344285,0.6211836 \backslash P G=C 01 \quad[X(C 18 H 18 N 105)] \backslash \backslash @$

\section{4,7-dicarboxy-TMAO•(42)}

$1 \backslash 1 \backslash G I N C-L C 133 \backslash F O p t \backslash U B 3 L Y P \backslash 6-31 G(d) \backslash C 18 H 18 N 105$ (2) \JOH501\31-May-2006\0

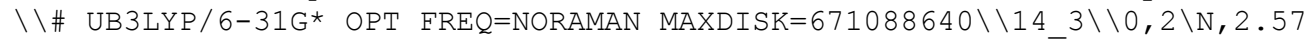
$18091496,0.0459700208,0.3801661282 \backslash 0,3.8551524207,0.0382 \overline{4} 12128,0.41764$ $38441 \backslash \mathrm{C},-0.2761372253,0.0766509412,0.3174666718 \backslash \mathrm{C},-1.7170822353,-0.056$ $5533536,0.3840754063 \backslash \mathrm{C},-2.3922302223,-0.643425261,-0.7134162159 \backslash \mathrm{C},-2.4$ $234633871,0.4078244858,1.5473738407 \backslash \mathrm{H},-3.4624531054,-0.7771568873,-0.6$ $611279578 \backslash \mathrm{C},-3.90394692,0.3062657965,1.6875224964 \backslash \mathrm{C},-1.6962339105,-1.0$ $311188628,-1.8255170568 \backslash \mathrm{C},-1.7097213797,0.9780828866,2.5867399191 \backslash \mathrm{H},-2$ $.2351629976,1.3450640346,3.4594914548 \backslash C,-0.2986138484,-0.8294214771,-1$ $.9394036941 \backslash \mathrm{C},-0.3146002213,1.064857387,2.5388993691 \backslash \mathrm{C}, 0.2287644377,-1$ $.1586086288,-3.3056922528 \backslash \mathrm{H}, 0.2024179344,1.490226542,3.3921674111 \backslash \mathrm{C}, 0$. $434548052,-0.3017337789,-0.8756415714 \backslash C, 0.4116270642,0.608764803,1.453$ $4722164 \backslash C, 1.9541787844,-0.0960219195,-0.9812255182 \backslash C, 1.9256546505,0.68$ $21376252,1.5541016362 \backslash C, 2.2736887015,1.1963897638,-1.7753463842 \backslash C, 2.40$ $63280386,2.1518224666,1.6561263137 \backslash C, 2.6679161047,-1.3164679445,-1.608$ $1803165 \backslash \mathrm{C}, 2.4152727648,-0.1150130075,2.7883493749 \backslash \mathrm{H}, 2.2960316727,-2.24$ $9948032,-1.1739866968 \backslash \mathrm{H}, 2.0470507703,-1.1449237448,2.7499980917 \backslash \mathrm{H}, 2.53$ $92300097,-1.3425691718,-2.6883613521 \backslash \mathrm{H}, 2.0654004162,0.3451227961,3.715$ $8938194 \backslash \mathrm{H}, 3.7338694025,-1.2337435472,-1.3967697913 \backslash \mathrm{H}, 3.5064938234,-0.1$ $319530219,2.788329122 \backslash \mathrm{H}, 1.7679814693,2.0619184734,-1.3353728229 \backslash \mathrm{H}, 2.01$ $82162967,2.7572770247,0.8321858107 \backslash \mathrm{H}, 3.3539218857,1.3676313231,-1.7465$ $5602 \backslash \mathrm{H}, 3.4983185294,2.1675127356,1.623883346 \backslash \mathrm{H}, 1.943038903,1.093921395$ $3,-2.8093867669 \backslash \mathrm{H}, 2.0724882076,2.6025059389,2.5953702757 \backslash 0,0.799927814$ $7,-0.405800727,-4.0676569395 \backslash 0,-0.0994792162,-2.4231116332,-3.66833755$ $17 \backslash \mathrm{H}, 0.2015086377,-2.5219283348,-4.5922914406 \backslash \mathrm{H},-2.2250487615,-1.48251$ $04862,-2.6582433755 \backslash 0,-4.6904289585,-0.2200307072,0.9211722798 \backslash 0,-4.35$ $52884849,0.8894349224,2.8328705012 \backslash \mathrm{H},-5.3207312387,0.7553067427,2.8198$ $515159 \backslash \backslash$ Version=IA32L-G03RevC.02 \State $=2-A \backslash H F=-1127.5951966 \backslash \mathrm{S} 2=0.75372$ $5 \backslash S 2-1=0 . \backslash S 2 A=0.75001 \backslash R M S D=7.461 e-09 \backslash R M S F=3.039 e-06 \backslash D i p o l e=-0.6556632$, $0.1262744,0.6058525 \backslash \mathrm{PG}=\mathrm{C} 01 \quad[\mathrm{X}(\mathrm{C} 18 \mathrm{H} 18 \mathrm{~N} 105)] \backslash \backslash @$

\section{4,7-dicarboxy-TMAO+}

$1 \backslash 1 \backslash$ GINC-LC127\FOpt \UB3LYP \6-31G (d) \C18H18N1O5 (1+) \JOH501\30-May-2006

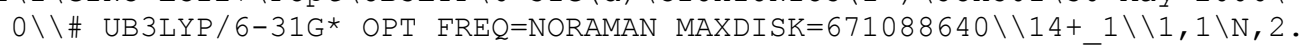
$2239438446,-1.2368069152,-0.3360640341 \backslash 0,3.4261824336,-1.2 \overline{6} 1099587,-0$. $3319256748 \backslash C,-0.1274052045,0.2419502676,-0.320385852 \backslash C,-1.1939383213,1$ $.2072914356,-0.3476514553 \backslash C,-2.1189756025,1.2018950655,0.7260967985 \backslash C$, $-1.2767726291,2.118815572,-1.4554658344 \backslash C,-2.3407159354,3.1796419304,-$ $1.5381892091 \backslash \mathrm{C},-1.974954435,0.3098618751,1.7581146847 \backslash \mathrm{C},-0.3615284046$, $2.028340263,-2.4903633639 \backslash \mathrm{H},-0.4182070154,2.726816699,-3.3157800321 \backslash \mathrm{C}$, $-0.8463103603,-0.5424446035,1.8678698256 \backslash C, 0.5996662411,1.0035274882$,- 
$2.5236192153 \backslash \mathrm{H}, 1.2297195461,0.9113856907,-3.3999984711 \backslash \mathrm{C}, 0.1139276753$, $-0.5555141767,0.8465207778 \backslash \mathrm{C}, 0.6801345425,0.0902243446,-1.4909601492 \backslash \mathrm{C}$ $, 1.4965951061,-1.2112765062,1.0029720145 \backslash C, 1.4913754301,-1.1981073948$, $-1.6540129845 \backslash \mathrm{C}, 1.5246521558,-2.7185721914,1.4126276598 \backslash \mathrm{C}, 0.5660877681$ $,-2.4312929938,-1.7800435944 \backslash C, 2.3809426354,-0.3665009309,1.9511076499$ $\backslash \mathrm{C}, 2.5239872657,-1.188467144,-2.7787608239 \backslash \mathrm{H}, 2.4792388344,0.6555012931$ $, 1.573447706 \backslash \mathrm{H}, 3.1831175567,-0.3181978636,-2.7337332846 \backslash \mathrm{H}, 1.895815877$, $-0.350155142,2.925627619 \backslash \mathrm{H}, 1.999137704,-1.1881068889,-3.7366311332 \backslash \mathrm{H}, 3$ $.3722812462,-0.8101912244,2.0565475133 \backslash \mathrm{H}, 3.140728456,-2.0893649701,-2$. $7373864327 \backslash \mathrm{H}, 0.8405244946,-3.3255165235,0.8174331854 \backslash \mathrm{H},-0.1970554456,-$ $2.4707697271,-1.0024780838 \backslash \mathrm{H}, 2.542365881,-3.1025468095,1.3045953517 \backslash \mathrm{H}$, $1.1490639262,-3.3571325572,-1.7774114343 \backslash \mathrm{H}, 1.2466552971,-2.783693247,2$ $.4626502841 \backslash \mathrm{H}, 0.0556214714,-2.3479013011,-2.7438935422 \backslash \mathrm{C},-0.7689590198$ $,-1.3065066406,3.1622668191 \backslash \mathrm{H},-2.7135818293,0.2880867254,2.5499110025 \backslash$ $\mathrm{H},-2.9490037451,1.8937466867,0.709827047 \backslash 0,0.2320785848,-1.5072760008$, $3.8221652138 \backslash 0,-1.9851325832,-1.7270979742,3.5498124025 \backslash \mathrm{H},-1.878364049$ $9,-2.1547077876,4.4223803502 \backslash 0,-3.3079657668,3.2793192625,-0.813322550$ $3 \backslash 0,-2.1044562596,4.0431274054,-2.5478084439 \backslash \mathrm{H},-2.8381698344,4.6874165$ $464,-2.5427694715 \backslash \backslash$ Version=IA32L-G03RevC.02 \State $=1-A \backslash H F=-1127.3487343$ $\backslash S 2=0 . \backslash S 2-1=0 . \backslash S 2 A=0 . \backslash R M S D=5.503 e-09 \backslash R M S F=1.640 e-05 \backslash D i p o l e=1.0100127,-$ $1.1256857,-1.1019535 \backslash \mathrm{PG}=\mathrm{C} 01 \quad[\mathrm{X}(\mathrm{C} 18 \mathrm{H} 18 \mathrm{~N} 105)] \backslash \backslash @$

\section{4,7-dicarboxy-TMAO-}

$1 \backslash 1 \backslash G I N C-A C 57 \backslash F O p t \backslash U B 3 L Y P \backslash 6-31 G(d) \backslash C 18 H 18 N 105$ (1-) \JOH501 \04-Dec-2006\0

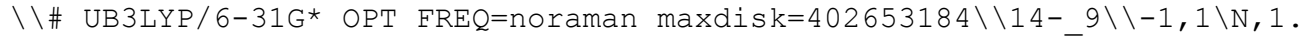
$6834073978,-1.8346707653,-0.437999334 \backslash 0,2.6483998274,-2.6 \overline{5} 73057393,-0$. $845791471 \backslash \mathrm{C},-0.3993968014,-0.116786354,0.0451929853 \backslash \mathrm{C},-1.6101722661,0$. $6703317553,0.019900328 \backslash C,-1.4811466193,2.0715574712,0.1514621629 \backslash C,-2$. $8869618485,0.0058339137,-0.119802305 \backslash C,-4.1457025176,0.72990473,-0.185$ $613584 \backslash C,-0.2393526205,2.6554553799,0.2568148255 \backslash C,-2.9034486116,-1.40$ $19769176,-0.1871091124 \backslash \mathrm{H},-3.8569128771,-1.9055654266,-0.3000752835 \backslash \mathrm{C}, 0$ $.9602043039,1.9185230386,0.114191121 \backslash \mathrm{C},-1.7515098363,-2.1547630381,-0$. $0375582173 \backslash \mathrm{H},-1.8359807607,-3.2354800085,-0.0042277751 \backslash \mathrm{C}, 0.8870906174$, $0.5076281374,-0.0341488027 \backslash C,-0.4954464154,-1.5519887266,0.1284469717 \backslash$ $\mathrm{C}, 2.0598455786,-0.3968928116,-0.4350180038 \backslash \mathrm{C}, 0.7334088245,-2.335510510$ $9,0.5514255527 \backslash \mathrm{C}, 3.3302898052,-0.2665363402,0.4478389669 \backslash \mathrm{C}, 1.115196162$ $5,-2.0980157449,2.0428347846 \backslash C, 2.3962876714,-0.089433223,-1.9189783251$ $\backslash \mathrm{C}, 0.5920671262,-3.8482933639,0.3334400247 \backslash \mathrm{H}, 1.4865994107,-0.170183094$ $8,-2.5235823408 \backslash \mathrm{H}, 0.2909344637,-4.0723317266,-0.6938079554 \backslash \mathrm{H}, 2.8245390$ $048,0.9066566443,-2.0241601105 \backslash \mathrm{H},-0.1468599006,-4.267199443,1.02662812$ $24 \backslash \mathrm{H}, 3.0982773744,-0.8585305695,-2.2512731989 \backslash \mathrm{H}, 1.560969974,-4.3188799$ $033,0.5042450231 \backslash \mathrm{H}, 3.0788706988,-0.2069335924,1.5114167518 \backslash \mathrm{H}, 1.1691859$ $099,-1.0316362907,2.2801577088 \backslash \mathrm{H}, 3.9083304588,-1.1783592262,0.26321101$ $03 \backslash \mathrm{H}, 2.0842201752,-2.5578024698,2.2672716807 \backslash \mathrm{H}, 3.9218429245,0.60777957$ $85,0.1817217842 \backslash \mathrm{H}, 0.3555914698,-2.54323108,2.6987489485 \backslash \mathrm{C}, 2.184877095$, $2.749348065,0.0786333472 \backslash \mathrm{H},-0.1717190668,3.7283759595,0.3944932799 \backslash \mathrm{H},-$ $2.3766629805,2.6761936518,0.1761224693 \backslash 0,3.2206816475,2.5910863209,-0$. $5498454533 \backslash 0,2.0683311176,3.8785042185,0.8603259603 \backslash \mathrm{H}, 2.8987995468,4.3$ $575842288,0.6858482149 \backslash 0,-4.3527328589,1.9420944976,-0.1124166813 \backslash 0,-5$ $.2471022702,-0.0962345173,-0.3564225698 \backslash \mathrm{H},-5.9847303347,0.5381932932$, $0.3778215008 \backslash \backslash$ Version=IA64L-G03RevD.01 \State $=1-A \backslash H F=-1127.6099189 \backslash \mathrm{S} 2=0$ $. \backslash \mathrm{S} 2-1=0 . \backslash \mathrm{S} 2 \mathrm{~A}=0 . \backslash \mathrm{RMSD}=6.814 \mathrm{e}-09 \backslash \mathrm{RMSF}=1.115 \mathrm{e}-05 \backslash \mathrm{Thermal}=0 . \backslash \mathrm{Dipole}=-0.64$ $75174,0.7883615,0.9140452 \backslash \mathrm{PG}=\mathrm{C} 01 \quad[\mathrm{X}(\mathrm{C} 18 \mathrm{H} 18 \mathrm{~N} 105)] \backslash \backslash \mathrm{Q}$

\section{4,8-dicarboxy-TMAO• (43)}

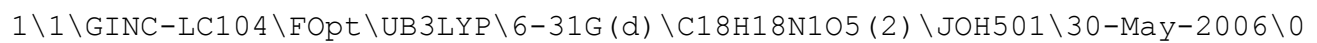

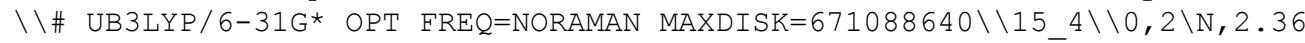
$34506376,-0.1382387702,0.0651279206 \backslash 0,3.6468685984,-0.16 \overline{3} 7286849,0.084$ $1022606 \backslash \mathrm{C},-0.4645428756,-0.0692669919,0.0268176423 \backslash \mathrm{C},-1.8991316778,-0$. $1770750196,0.1050963021 \backslash \mathrm{C},-2.6222863986,-0.7162369143,-0.9844949153 \backslash \mathrm{C}$, $-2.5929554293,0.2520669453,1.2602467804 \backslash \mathrm{H},-3.7014300159,-0.8147155699$, $-0.906431725 \backslash \mathrm{C},-1.9641752589,-1.1053652081,-2.1171527206 \backslash \mathrm{C},-1.90266038$ $71,0.7807735804,2.3287385831 \backslash C,-2.5781645035,1.2666685361,3.5605305832$ 
$\backslash C,-0.5572924415,-0.9402166368,-2.2391127229 \backslash C,-0.4928701615,0.8578862$ $922,2.2746423427 \backslash \mathrm{C},-0.0525925093,-1.279010995,-3.6126450018 \backslash \mathrm{H}, 0.011009$ $4251,1.2597352155,3.1458919776 \backslash \mathrm{C}, 0.2130020921,-0.4443436549,-1.1825895$ $506 \backslash C, 0.226517309,0.4331807718,1.1761955692 \backslash C, 1.7354930351,-0.27632260$ $36,-1.2957794005 \backslash C, 1.74246099,0.5131613368,1.2495079555 \backslash C, 2.0864095984$ $, 1.0016105317,-2.0996961901 \backslash \mathrm{C}, 2.2182393863,1.9849906683,1.3233260259 \backslash \mathrm{C}$ $, 2.4148985361,-1.5197616518,-1.9147347399 \backslash \mathrm{C}, 2.2637415719,-0.2648798343$ $, 2.4811790021 \backslash \mathrm{H}, 2.0404041537,-2.4380983939,-1.4512195959 \backslash \mathrm{H}, 1.906181268$ $8,-1.2990801989,2.4605921054 \backslash \mathrm{H}, 2.2570553321,-1.5664536076,-2.990625783$ $3 \backslash \mathrm{H}, 1.9229989223,0.2024380207,3.4084904887 \backslash \mathrm{H}, 3.4869091124,-1.449021996$ $2,-1.7314627286 \backslash \mathrm{H}, 3.354841701,-0.2700224596,2.4658244108 \backslash \mathrm{H}, 1.598621946$ $3,1.8814109559,-1.6681429627 \backslash \mathrm{H}, 1.8139961502,2.5784782661,0.4982561665 \backslash$ $\mathrm{H}, 3.1698981266,1.1480602293,-2.0657577284 \backslash \mathrm{H}, 3.3098534901,2.0088798293$, $1.2761496121 \backslash \mathrm{H}, 1.758970897,0.8986821192,-3.1345040957 \backslash \mathrm{H}, 1.8924372781,2$ $.443634039,2.2617787547 \backslash 0,0.5506283041,-0.5475225985,-4.3700102023 \backslash 0,-$ $0.4404602514,-2.5242085888,-3.9843239667 \backslash \mathrm{H},-0.1442378686,-2.6308987513$ $,-4.9089443522 \backslash \mathrm{H},-2.5122910466,-1.5271804045,-2.9523049571 \backslash \mathrm{H},-3.673807$ $1003,0.1651550407,1.2898436665 \backslash 0,-2.0065226509,1.7368422126,4.52387686$ $77 \backslash 0,-3.9284974951,1.1368710588,3.5140695324 \backslash \mathrm{H},-4.2562435306,1.4834869$ $489,4.3645021008 \backslash \backslash$ Version=IA32L-G03RevC.02 \State $=2-A \backslash H F=-1127.6002716 \backslash$ $\mathrm{S} 2=0.753727 \backslash \mathrm{S} 2-1=0 . \backslash \mathrm{S} 2 \mathrm{~A}=0.75001 \backslash \mathrm{RMSD}=7.372 \mathrm{e}-09 \backslash \mathrm{RMSF}=8.000 e-06 \backslash \mathrm{Dipole}=-$ $1.487632,-0.4096929,-0.3972192 \backslash \mathrm{PG}=\mathrm{C} 01 \quad[\mathrm{X}(\mathrm{C} 18 \mathrm{H} 18 \mathrm{~N} 105)] \backslash \backslash \mathrm{Q}$

\section{4,8-dicarboxy-TMAO+}

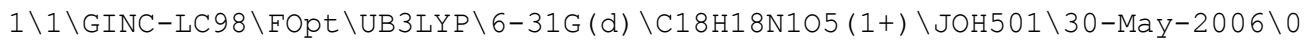

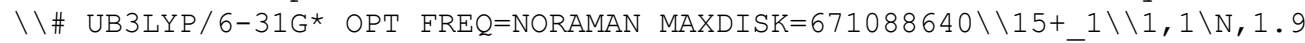
$689451999,-1.1967169758,-0.0416247643 \backslash 0,3.1702398423,-1.2 \overline{1} 53174781,-0$. $0254133444 \backslash \mathrm{C},-0.3793887688,0.2615532614,-0.0208377799 \backslash \mathrm{C},-1.4380762236$, $1.2259167571,-0.0460438249 \backslash \mathrm{C},-2.359223774,1.2588616749,1.0251368695 \backslash \mathrm{C}$, $-1.5521637159,2.1201524357,-1.1409945529 \backslash \mathrm{C},-2.2286865348,0.3783843939$, $2.0684631514 \backslash C,-0.6689017659,2.054012423,-2.1988711794 \backslash C,-0.7273368851$ $, 2.9802532924,-3.3726754989 \backslash \mathrm{C},-1.1095048526,-0.4923347715,2.1712331252$ $\backslash \mathrm{C}, 0.3137089198,1.0372863185,-2.2319069072 \backslash \mathrm{H}, 0.927845021,0.9741493777$, $-3.1220750909 \backslash \mathrm{C},-0.1449524407,-0.52661078,1.1496587314 \backslash \mathrm{C}, 0.4216030183$, $0.1278978861,-1.2004394645 \backslash \mathrm{C}, 1.2325672165,-1.1950795094,1.3003394599 \backslash \mathrm{C}$ $, 1.2499732419,-1.1468135907,-1.3709226942 \backslash C, 1.2474735578,-2.7085471832$ $, 1.6824929065 \backslash C, 0.3439418069,-2.3906843597,-1.5198347421 \backslash C, 2.119717271$ $4,-0.3725543821,2.2645709122 \backslash C, 2.2950230279,-1.1052492366,-2.482796507$ $9 \backslash \mathrm{H}, 2.2306636529,0.6537768425,1.9025447175 \backslash \mathrm{H}, 2.9388357904,-0.224847607$ $9,-2.4167537884 \backslash \mathrm{H}, 1.631068312,-0.366262581,3.2372379028 \backslash \mathrm{H}, 1.7807475308$ $,-1.0938563101,-3.4462841007 \backslash \mathrm{H}, 3.1058930986,-0.8276719236,2.3684653216$ $\backslash \mathrm{H}, 2.924782276,-1.9975008675,-2.4503821553 \backslash \mathrm{H}, 0.5523859372,-3.297531755$ $, 1.0822309243 \backslash \mathrm{H},-0.4283523509,-2.446927552,-0.7519975866 \backslash \mathrm{H}, 2.259791459$ $,-3.1031261875,1.5621601797 \backslash \mathrm{H}, 0.9372025521,-3.3100393511,-1.518841366 \backslash$ $\mathrm{H}, 0.9744725873,-2.7880398621,2.732973566 \backslash \mathrm{H},-0.1561363845,-2.3038713639$ $,-2.4888450662 \backslash \mathrm{C},-1.044643901,-1.2666773108,3.4610706527 \backslash \mathrm{H},-2.95933987$ $47,0.3715209028,2.8673602779 \backslash \mathrm{H},-3.1777869418,1.9719221999,1.003946994 \backslash$ $0,-0.0429446841,-1.5226427677,4.0999628673 \backslash 0,-2.2722595694,-1.63225285$ $65,3.8692703938 \backslash \mathrm{H},-2.1684336377,-2.0760508308,4.7340559446 \backslash \mathrm{H},-2.343096$ $4173,2.8627494795,-1.1373917792 \backslash 0,0.0222412443,2.9002771929,-4.3212229$ $781 \backslash 0,-1.6988835613,3.9045627007,-3.2525841559 \backslash \mathrm{H},-1.6670803726,4.46300$ $7978,-4.052989749 \backslash \backslash$ Version=IA32L-G03RevC.02 $\backslash$ State $=1-A \backslash H F=-1127.3544378$ $\backslash S 2=0 . \backslash S 2-1=0 . \backslash S 2 A=0 . \backslash R M S D=9.005 e-09 \backslash R M S F=1.177 e-05 \backslash D i p o l e=-0.2329415$, $-0.99177,0.2728842 \backslash P G=C 01 \quad[X(C 18 H 18 N 105)] \backslash \backslash @$

\section{4,8-dicarboxy-TMAO-}

$1 \backslash 1 \backslash G I N C-A C 56 \backslash F O p t \backslash U B 3 L Y P \backslash 6-31 G(d) \backslash C 18 H 18 N 105$ (1-) \JOH501 \07-Dec-2006\0

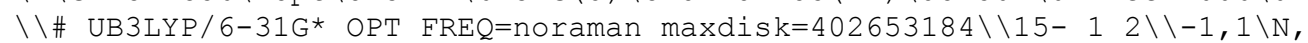
$-0.0051724676,0.0003353052,-0.00604676 \backslash 0,1.3446609543,-0 . \overline{0} 4 \overline{1} 4876838,-0$ $.0005936897 \backslash C,-2.7479395869,0.4764711223,-0.0031573171 \backslash C,-4.1959546201$ $, 0.4722164154,0.0827572635 \backslash \mathrm{C},-4.952629532,0.2487152888,-1.1020518548 \backslash \mathrm{C}$ $,-4.8476787472,0.6917700695,1.3023924218 \backslash \mathrm{H},-6.0376229048,0.2078747283$, $-1.0330222731 \backslash \mathrm{C},-4.3245987919,0.1148705816,-2.3015518545 \backslash \mathrm{C},-4.12721566$ 
$87,0.9245268406,2.4727110151 \backslash C,-4.855386754,1.1780373664,3.7139997591 \backslash$ $C,-2.9000354836,0.2220974775,-2.4245380242 \backslash C,-2.7103375662,0.885673204$ $5,2.4070850284 \backslash \mathrm{C},-2.4345152729,0.2444149128,-3.829611685 \backslash \mathrm{H},-2.15191052$ $32,1.0339687609,3.3234408642 \backslash C,-2.092049613,0.3598842932,-1.269787028 \backslash$ $C,-2.0306890669,0.6556501846,1.2294187381 \backslash C,-0.5535514891,0.3837549834$ $,-1.3390354628 \backslash C,-0.5237246243,0.5713262114,1.2493528635 \backslash C,-0.00982087$ $17,1.7660622997,-1.7987490164 \backslash \mathrm{C}, 0.107202326,1.9609133742,1.5358411127 \backslash$ $\mathrm{C},-0.0025804403,-0.732256128,-2.2689404706 \backslash \mathrm{C},-0.0631918989,-0.42349255$ $82,2.3468386187 \backslash \mathrm{H},-0.5309379124,-1.6745923217,-2.0818496313 \backslash \mathrm{H},-0.58217$ $60874,-1.3801169905,2.2252209288 \backslash \mathrm{H},-0.0711640327,-0.4749464009,-3.3244$ $383401 \backslash \mathrm{H},-0.2520777971,-0.0426089015,3.3558760719 \backslash \mathrm{H}, 1.0437221509,-0.86$ $39466839,-1.9890223416 \backslash \mathrm{H}, 1.0066623576,-0.585016905,2.2004440855 \backslash \mathrm{H},-0.5$ $015696897,2.5815646461,-1.2565123468 \backslash \mathrm{H},-0.3474845389,2.7468679805,0.92$ $58340877 \backslash \mathrm{H}, 1.0589604416,1.7808527917,-1.5663652694 \backslash \mathrm{H}, 1.1690570572,1.87$ $15057341,1.2810256562 \backslash \mathrm{H},-0.1687861942,1.922006405,-2.8657775756 \backslash \mathrm{H},-0.0$ $034335997,2.2441034334,2.5892290065 \backslash 0,-1.556730282,0.916193857,-4.3499$ $561786 \backslash 0,-3.1924113965,-0.5778091517,-4.638902504 \backslash \mathrm{H},-2.8371595092,-0.4$ $035412931,-5.529465433 \backslash \mathrm{H},-4.9055172252,-0.0372864275,-3.203697298 \backslash \mathrm{H},-5$ $.9333580435,0.691973435,1.3399406674 \backslash 0,-6.0736810333,1.2034980422,3.85$ $32244348 \backslash 0,-4.0405625834,1.4039548265,4.7993609994 \backslash \mathrm{H},-4.6659494389,1.5$ $50946874,5.5309787312 \backslash \backslash$ Version=IA64L-G03RevD.01 \State=1-A \HF=-1127.617 $3352 \backslash \mathrm{S} 2=0 . \backslash \mathrm{S} 2-1=0 . \backslash \mathrm{S} 2 \mathrm{~A}=0 . \backslash \mathrm{RMSD}=2.855 \mathrm{e}-09 \backslash \mathrm{RMSF}=3.176 \mathrm{e}-06 \backslash \mathrm{Thermal}=0 . \backslash \mathrm{Dip}$ ole $=-1.5619962,0.25783,-0.1609087 \backslash \mathrm{PG}=\mathrm{C} 01 \quad[\mathrm{X}(\mathrm{C} 18 \mathrm{H} 18 \mathrm{N1O5})] \backslash \backslash \mathrm{Q}$

\section{4,9-dicarboxy-TMAO• (44)}

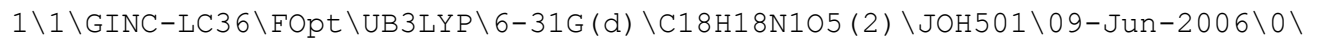

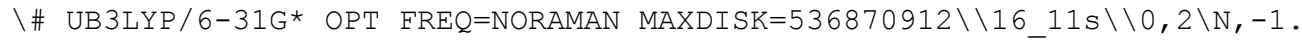
$9662850578,0.0868031529,0 . \backslash 0,-3.2498657988,0.0859908942,0 . \backslash C, 0.8465680$ $963,0.1243020707,0 . \backslash \mathrm{C}, 2.2665516952,0.3726286233,0 . \backslash \mathrm{C}, 2.9732060909,0.50$ $4884505,-1.216377719 \backslash \mathrm{C}, 2.9732060909,0.504884505,1.216377719 \backslash \mathrm{H}, 4.040851$ $5799,0.7034782416,-1.1853174723 \backslash \mathrm{H}, 4.0408515799,0.7034782416,1.18531747$ $23 \backslash \mathrm{C}, 2.316536923,0.3911455612,-2.4072246335 \backslash \mathrm{C}, 2.316536923,0.3911455612$ $, 2.4072246335 \backslash \mathrm{H}, 2.8512383979,0.5023710961,-3.3440679537 \backslash \mathrm{H}, 2.8512383979$ $, 0.5023710961,3.3440679537 \backslash \mathrm{C}, 0.9239631052,0.119332369,-2.441352357 \backslash \mathrm{C}, 0$ $.9239631052,0.119332369,2.441352357 \backslash C, 0.1705687692,0.0030889848,-1.268$ $4686858 \backslash \mathrm{C}, 0.1705687692,0.0030889848,1.2684686858 \backslash \mathrm{C}, 0.4137804319,-0.061$ $0463826,-3.8435901155 \backslash \mathrm{C}, 0.4137804319,-0.0610463826,3.8435901155 \backslash 0,0.73$ $14877367,1.008301779,-4.6171172577 \backslash 0,0.7314877367,1.008301779,4.617117$ $2577 \backslash \mathrm{H}, 0.4431979342,0.7766784364,-5.521138818 \backslash \mathrm{H}, 0.4431979342,0.7766784$ $364,5.521138818 \backslash 0,-0.1315359934,-1.0395489106,-4.3092078816 \backslash 0,-0.13153$ $59934,-1.0395489106,4.3092078816 \backslash C,-1.3443767697,-0.2118222116,-1.3306$ $698056 \backslash \mathrm{C},-1.3443767697,-0.2118222116,1.3306698056 \backslash \mathrm{C},-2.0196022274,0.78$ $26684544,-2.3061633654 \backslash \mathrm{C},-2.0196022274,0.7826684544,2.3061633654 \backslash \mathrm{H},-1$. $6707848564,1.8034734404,-2.1212016968 \backslash \mathrm{H},-1.6707848564,1.8034734404,2.1$ $212016968 \backslash \mathrm{H},-3.0962980676,0.7459960357,-2.141424785 \backslash \mathrm{H},-3.0962980676,0$. $7459960357,2.141424785 \backslash \mathrm{H},-1.8270691119,0.5196877725,-3.3449769417 \backslash \mathrm{H},-1$ $.8270691119,0.5196877725,3.3449769417 \backslash C,-1.7029624168,-1.6728818236,-1$ $.7079101636 \backslash \mathrm{C},-1.7029624168,-1.6728818236,1.7079101636 \backslash \mathrm{H},-1.1818146525$ $,-2.3878255017,-1.0655981494 \backslash \mathrm{H},-1.1818146525,-2.3878255017,1.065598149$ $4 \backslash \mathrm{H},-1.4225307017,-1.8781439388,-2.7403304535 \backslash \mathrm{H},-1.4225307017,-1.87814$ $39388,2.7403304535 \backslash \mathrm{H},-2.7809863828,-1.8065219636,-1.5817074532 \backslash \mathrm{H},-2.78$ $09863828,-1.8065219636,1.5817074532 \backslash \backslash$ Version=IA32L-G03RevC.02 $\backslash$ State $=2-$ $A^{\prime} \backslash H F=-1127.5824087 \backslash S 2=0.753692 \backslash S 2-1=0 . \backslash S 2 A=0.750009 \backslash R M S D=4.830 e-09 \backslash R M$ $\mathrm{SF}=6.997 \mathrm{e}-06 \backslash \mathrm{Dipole}=1.5697995,0.6840914,0 . \backslash \mathrm{PG}=\mathrm{CS} \quad[\mathrm{SG}(\mathrm{C} 2 \mathrm{~N} 1 \mathrm{O} 1), \mathrm{X}(\mathrm{C} 16 \mathrm{H} 18 \mathrm{O}$ 4) $] \backslash \backslash 0$

\section{4,9-dicarboxy-TMAO+}

$1 \backslash 1 \backslash G I N C-L C 96 \backslash F O p t \backslash U B 3 L Y P \backslash 6-31 G(d) \backslash C 18 H 18 N 105(1+) \backslash J O H 501 \backslash 04-J u n-2006 \backslash 0$

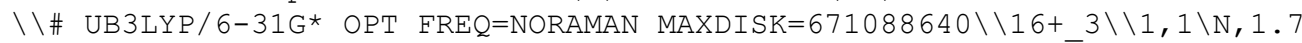
$317004492,-0.8479433334,0.0866977072 \backslash 0,2.9294902218,-0.79 \overline{5} 0608554,0.14$ $99872253 \backslash \mathrm{C},-0.5777878664,0.6010476549,0.0122098271 \backslash \mathrm{C},-1.6098897381,1.5$ $970242375,0.0122625617 \backslash C,-2.5550533641,1.6287034574,1.0632655481 \backslash C,-1$. $6847603974,2.5293915505,-1.0480449519 \backslash C,-2.4930940291,0.7051968396,2.0$ 
$709146937 \backslash C,-0.8378657844,2.4303404299,-2.1215993578 \backslash C,-1.3990734299,-$ $0.1972656173,2.1704394678 \backslash \mathrm{C}, 0.0702533792,1.3460291131,-2.2287256673 \backslash \mathrm{C}$, $0.6177379906,1.1741240158,-3.6211958661 \backslash C,-0.4018513103,-0.2131789751$, $1.1837536491 \backslash \mathrm{C}, 0.2137345071,0.4365899262,-1.1805145322 \backslash \mathrm{C}, 0.9565711054$, $-0.8934467247,1.3875324003 \backslash C, 1.0640730193,-0.8479077264,-1.2880430587 \backslash$ $C, 0.9583989669,-2.4218801568,1.7205462212 \backslash C, 0.1542792332,-2.0840549543$ $,-1.4608984888 \backslash \mathrm{C}, 1.7974847845,-0.109334389,2.4222434301 \backslash \mathrm{C}, 2.2072821358$ $,-0.8914000633,-2.3045175314 \backslash \mathrm{H}, 1.9424623026,0.9241213603,2.094145985 \backslash \mathrm{H}$ $, 2.7825758508,0.0381626787,-2.3303044601 \backslash \mathrm{H}, 1.2559469958,-0.121535192,3$ $.3660428761 \backslash \mathrm{H}, 1.8006987316,-1.0908793381,-3.2918488565 \backslash \mathrm{H}, 2.7710203621$, $-0.5793536131,2.5697028321 \backslash \mathrm{H}, 2.8917579147,-1.7035184998,-2.0487240442 \backslash$ $\mathrm{H}, 0.2787362427,-2.9905706691,1.0854394631 \backslash \mathrm{H},-0.6607106746,-2.120267037$ $4,-0.7373801509 \backslash \mathrm{H}, 1.9732964413,-2.8143997854,1.6151602153 \backslash \mathrm{H}, 0.73461522$ $41,-3.0105260196,-1.4184621755 \backslash \mathrm{H}, 0.6581430745,-2.5293747785,2.76110698$ $88 \backslash \mathrm{H},-0.2840958172,-1.9937469955,-2.4591061046 \backslash \mathrm{C},-1.4022396399,-1.0287$ $872534,3.4263261169 \backslash \mathrm{H},-3.2509454323,0.686722015,2.8439067231 \backslash \mathrm{H},-3.3454$ $221138,2.3728537037,1.0421663776 \backslash 0,-0.4386025182,-1.3219023517,4.10644$ $30553 \backslash 0,-2.652543577,-1.4012124248,3.7521152925 \backslash \mathrm{H},-2.5976270569,-1.883$ $8827066,4.600400559 \backslash \mathrm{H},-2.434566954,3.3138856938,-1.0080399603 \backslash \mathrm{H},-0.904$ $0170926,3.1369710224,-2.9416152889 \backslash 0,0.3696407688,0.2230196883,-4.3289$ $457925 \backslash 0,1.3220669933,2.2472715969,-4.0156885721 \backslash \mathrm{H}, 1.556616372,2.11686$ $80835,-4.9564913679 \backslash \backslash$ Version=IA32L-G03RevC.02 $\backslash$ State $=1-A \backslash H F=-1127.33724$ $39 \backslash S 2=0 . \backslash S 2-1=0 . \backslash S 2 A=0 . \backslash R M S D=5.177 e-09 \backslash R M S F=2.303 e-06 \backslash D i p o l e=-0.071910$ $8,-0.0745734,0.032307 \backslash \mathrm{PG}=\mathrm{C} 01 \quad[\mathrm{X}(\mathrm{C} 18 \mathrm{H} 18 \mathrm{~N} 105)] \backslash \backslash @$

\section{4,9-dicarboxy-TMAO-}

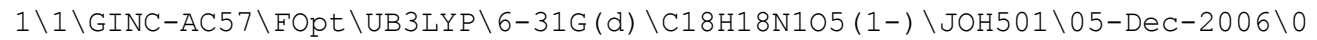

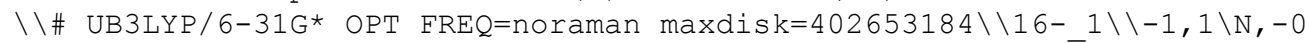
$.084848152,0.0016149674,-0.2172344165 \backslash 0,1.2859136627,0.0258820831,-0.1$ $347902025 \backslash \mathrm{C},-2.8359026214,-0.0033635573,0.1131180665 \backslash \mathrm{C},-4.2701712302,0$ $.0095075449,-0.0699063595 \backslash \mathrm{C},-4.9818952209,1.2247646334,-0.0874423212 \backslash \mathrm{C}$ $,-4.9717167205,-1.2109996657,-0.2454375022 \backslash \mathrm{H},-6.059234998,1.1990686337$ $,-0.238324961 \backslash \mathrm{H},-6.0474622208,-1.1808616861,-0.4061298252 \backslash \mathrm{C},-4.3264223$ $805,2.4181032519,0.0863870398 \backslash C,-4.3043125695,-2.3977196868,-0.1946486$ $547 \backslash \mathrm{H},-4.8724769991,3.3559370952,0.0800784306 \backslash \mathrm{H},-4.8414741103,-3.33221$ $75847,-0.3160485784 \backslash \mathrm{C},-2.9237010285,2.4371685138,0.2903382882 \backslash \mathrm{C},-2.900$ $1408647,-2.4472788639,0.0503158845 \backslash C,-2.159627224,1.2624584437,0.28110$ $22073 \backslash \mathrm{C},-2.1388550271,-1.262341797,0.1572129519 \backslash \mathrm{C},-2.3944719279,3.8079$ $587038,0.5627918175 \backslash \mathrm{C},-2.3919857125,-3.8319661895,0.2241127235 \backslash 0,-2.78$ $59342426,4.6994488755,-0.4010742771 \backslash 0,-2.9388921213,-4.695497208,-0.70$ $06570509 \backslash \mathrm{H},-2.4534448203,5.559053101,-0.0805562809 \backslash \mathrm{H},-2.5985585549,-5$. $5673096583,-0.4275261978 \backslash 0,-1.7802242346,4.1988342193,1.5354720524 \backslash 0,-$ $1.6630016669,-4.2833979854,1.0902877995 \backslash C,-0.6415896131,1.2759477301,0$ $.3246140471 \backslash \mathrm{C},-0.6095541222,-1.3000339464,0.2771207566 \backslash \mathrm{C},-0.0777645307$ $, 2.3363682226,-0.6605113971 \backslash C, 0.0120604671,-2.3132073156,-0.7273819779$ $\backslash \mathrm{H},-0.5850681569,2.2646982935,-1.6287933887 \backslash \mathrm{H},-0.4698000905,-2.2215618$ $87,-1.7078327882 \backslash \mathrm{H}, 0.9729357684,2.0684510248,-0.7932400937 \backslash \mathrm{H}, 1.0556182$ $599,-2.0084435724,-0.8261526326 \backslash \mathrm{H},-0.1552164155,3.3586619988,-0.282939$ $1202 \backslash \mathrm{H},-0.0395721082,-3.3472246422,-0.3854756126 \backslash \mathrm{C},-0.1017145789,1.552$ $9664047,1.7525566847 \backslash \mathrm{C},-0.1223327108,-1.6509663453,1.7134790433 \backslash \mathrm{H},-0.6$ $629436977,1.0072745861,2.5170218386 \backslash \mathrm{H},-0.7083966159,-1.1310290052,2.47$ $85845037 \backslash \mathrm{H},-0.1442435856,2.6144472234,2.0017882746 \backslash \mathrm{H},-0.1892234902,-2$. $7209188945,1.9112939115 \backslash \mathrm{H}, 0.9354017315,1.1965902936,1.7294234791 \backslash \mathrm{H}, 0.9$ $180244753,-1.3180062483,1.7677838414 \backslash \backslash$ Version=IA64L-G03RevD.01 $\backslash$ State $=1$ $-\mathrm{A} \backslash \mathrm{HF}=-1127.5928481 \backslash \mathrm{S} 2=0 . \backslash \mathrm{S} 2-1=0 . \backslash \mathrm{S} 2 \mathrm{~A}=0 . \backslash \mathrm{RMSD}=2.570 \mathrm{e}-09 \backslash \mathrm{RMSF}=6.767 \mathrm{e}-06$ $\backslash$ Thermal $=0 . \backslash$ Dipole $=-2.9984701,0.2907484,-0.219177 \backslash \mathrm{PG}=\mathrm{C} 01 \quad[\mathrm{X}(\mathrm{C} 18 \mathrm{H} 18 \mathrm{~N} 105$ ) $] \backslash \backslash$ e

\section{5,6-dicarboxy-TMAO• $(45)$}

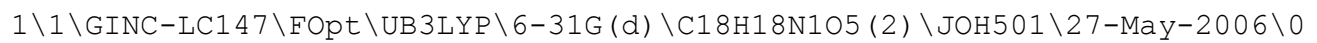

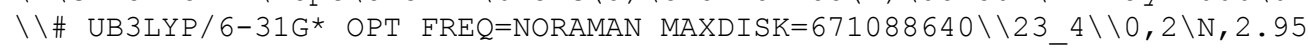
$69762145,-0.3901186849,0.5144606228 \backslash 0,4.2408973053,-0.38 \overline{3} 4437001,0.490$ $1932491 \backslash C, 0.1104254036,-0.3631388445,0.5922931198 \backslash C,-1.3292872174,-0.4$ 
$487220216,0.5693440263 \backslash \mathrm{C},-2.001117974,-1.1288961054,1.6201631553 \backslash \mathrm{C},-2$. $0546952519,0.1507839191,-0.5020230097 \backslash \mathrm{H},-3.0837264086,-1.172013738,1.6$ $192791229 \backslash \mathrm{C},-3.5547115977,0.0033265966,-0.5264286974 \backslash \mathrm{C},-1.2887048879,-$ $1.6958456078,2.6477771198 \backslash \mathrm{C},-1.3753866536,0.8456840422,-1.4910834234 \backslash \mathrm{H}$ $,-1.8067253893,-2.2058588296,3.4547842403 \backslash \mathrm{C},-2.134578939,1.5424944308$, $-2.5652783176 \backslash \mathrm{C}, 0.1178107684,-1.6161307288,2.6665890318 \backslash \mathrm{C}, 0.0357171091$ $, 0.9321643777,-1.4502920631 \backslash \mathrm{H}, 0.6508938732,-2.0715595831,3.4949985954 \backslash$ $\mathrm{H}, 0.5255537349,1.4906008273,-2.2373922704 \backslash \mathrm{C}, 0.8244100967,-0.9730888548$ $, 1.6666559842 \backslash \mathrm{C}, 0.7804434417,0.3386374153,-0.4536628202 \backslash \mathrm{C}, 2.2958052303$ $, 0.4974576404,-0.48613255 \backslash \mathrm{C}, 2.3439823949,-0.9089993567,1.7723577236 \backslash \mathrm{C}$, $2.6882345348,1.9624825711,-0.1713804341 \backslash \mathrm{C}, 2.7639578431,0.0215462762,2$. $936999452 \backslash \mathrm{C}, 2.8639873116,0.0831294215,-1.8619992262 \backslash \mathrm{C}, 2.9420570805,-2$. $3184803219,1.9831194178 \backslash \mathrm{H}, 2.5429314968,-0.9306896676,-2.1204889111 \backslash \mathrm{H}, 2$ $.6010913688,-3.0015455465,1.1989871551 \backslash \mathrm{H}, 2.5255578456,0.7652198219,-2$. $646130747 \backslash \mathrm{H}, 2.6468225395,-2.7273324335,2.9528991406 \backslash \mathrm{H}, 3.9539936769,0.1$ $070371126,-1.8205921555 \backslash \mathrm{H}, 4.0305940899,-2.2541329607,1.9453311069 \backslash \mathrm{H}, 2$. $2583214315,2.2923393129,0.7795825413 \backslash \mathrm{H}, 2.3220344662,1.0168822661,2.827$ $6461651 \backslash \mathrm{H}, 3.7773748616,2.0370036135,-0.1131604729 \backslash \mathrm{H}, 3.853068891,0.1155$ $849131,2.9473134829 \backslash \mathrm{H}, 2.325050643,2.6322871718,-0.9567778697 \backslash \mathrm{H}, 2.43195$ $24326,-0.3916060393,3.894424633 \backslash 0,-3.338138675,1.704192876,-2.58128705$ $11 \backslash 0,-1.3353120867,2.0157224105,-3.5550069609 \backslash \mathrm{H},-1.9320550949,2.466519$ $717,-4.1808889621 \backslash 0,-4.3122467638,0.525072545,0.2598038024 \backslash 0,-3.950775$ $8798,-0.8749957147,-1.4731676403 \backslash \mathrm{H},-4.9270513185,-0.8767215941,-1.4474$ $332819 \backslash \backslash$ Version=IA32L-G03RevC .02 \State $=2-A \backslash H F=-1127.6001333 \backslash \mathrm{S} 2=0.75374$ $4 \backslash \mathrm{S} 2-1=0 . \backslash \mathrm{S} 2 \mathrm{~A}=0.75001 \backslash \mathrm{RMSD}=5.377 \mathrm{e}-09 \backslash \mathrm{RMSF}=1.016 \mathrm{e}-05 \backslash \mathrm{Dipole}=0.1075746,-$ $0.3042491,-0.0340908 \backslash \mathrm{PG}=\mathrm{C} 01 \quad[\mathrm{X}(\mathrm{C} 18 \mathrm{H} 18 \mathrm{~N} 105)] \backslash \backslash @$

\section{5,6-dicarboxy-TMAO+}

$1 \backslash 1 \backslash$ GINC-LC150 \FOpt \UB3LYP \6-31G (d) \C18H18N105 (1+) \JOH501 \30-May-2006\ $0 \backslash \backslash \#$ UB3LYP/6-31G* OPT FREQ=NORAMAN MAXDISK $=671088640 \backslash \backslash 23+2 \backslash \backslash 1,1 \backslash \mathrm{N}, 2$. $6417024358,-1.3703561174,-0.5794759974 \backslash 0,3.8425260442,-1.4 \overline{3} 7327614,-0$. $5361308419 \backslash \mathrm{C}, 0.2805425518,0.1216860186,-0.653526097 \backslash \mathrm{C},-0.7948957119,1$. $0676967648,-0.6674664188 \backslash \mathrm{C},-1.6865450809,1.1269716953,0.4518854481 \backslash \mathrm{C},-$ $0.9217809693,1.9210596953,-1.7973859641 \backslash \mathrm{C},-2.7857608902,2.1622074736,0$ $.4332756443 \backslash \mathrm{H},-1.7343020897,2.6360641783,-1.8337895397 \backslash \mathrm{C},-1.5068837772$ $, 0.2552222632,1.5178663516 \backslash \mathrm{C},-0.0376728767,1.834572597,-2.8531483355 \backslash \mathrm{C}$ $,-2.4235725131,0.1328666452,2.7020834108 \backslash \mathrm{H},-0.1454845692,2.4990320721$, $-3.70429534 \backslash \mathrm{C},-0.3745838417,-0.5918477044,1.5594260239 \backslash \mathrm{C}, 0.971426058,0$ $.8516553412,-2.8706215262 \backslash \mathrm{H}, 1.6025721073,0.7632236906,-3.7472676704 \backslash \mathrm{C}$, $0.5296749743,-0.6384452967,0.5214218562 \backslash C, 1.0956759763,-0.0334485631,-$ $1.8124667565 \backslash C, 1.8791912016,-1.306025999,0.7326021022 \backslash \mathrm{C}, 1.9437647122,-$ $1.3053756351,-1.9303611882 \backslash \mathrm{C}, 1.7986502652,-2.7773552234,1.2310774476 \backslash \mathrm{C}$ $, 1.0531484969,-2.5606320453,-2.083254278 \backslash C, 2.7411786401,-0.4672689898$, $1.7071829557 \backslash \mathrm{C}, 3.0176839606,-1.2702493759,-3.0146981695 \backslash \mathrm{H}, 2.8687174263$ $, 0.5523137823,1.332758576 \backslash \mathrm{H}, 3.6552346737,-0.3856519948,-2.938672157 \backslash \mathrm{H}$, $2.2196540759,-0.417949148,2.664862158 \backslash \mathrm{H}, 2.52795497,-1.2701191427,-3.99$ $09962599 \backslash \mathrm{H}, 3.7204640185,-0.923437956,1.8612491862 \backslash \mathrm{H}, 3.6506402312,-2.15$ $89480752,-2.9611357838 \backslash \mathrm{H}, 1.1826204149,-3.4064573514,0.5869340942 \backslash \mathrm{H}, 0.2$ $610400532,-2.6096758565,-1.3348980073 \backslash \mathrm{H}, 2.8047461821,-3.1984417298,1.3$ $062735879 \backslash \mathrm{H}, 1.6530531244,-3.4756083713,-2.0519152997 \backslash \mathrm{H}, 1.356323371,-2$. $7689542554,2.229839596 \backslash \mathrm{H}, 0.576603436,-2.4943415442,-3.0658416027 \backslash \mathrm{O},-3$. $621108215,2.2463101066,-0.4359763776 \backslash 0,-2.6633925844,3.0195663758,1.46$ $16587439 \backslash \mathrm{H},-3.4065115463,3.6520673226,1.3994856676 \backslash \mathrm{H},-0.2351981237,-1$. $1645340535,2.4703457261 \backslash 0,-2.0718176413,-0.3441917474,3.7587115412 \backslash 0,-$ $3.6772213678,0.5411783891,2.4428761016 \backslash \mathrm{H},-4.1973817479,0.4198892046,3$.

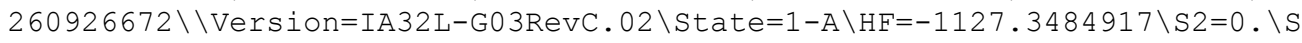
$2-1=0 . \backslash S 2 A=0 . \backslash R M S D=2.519 e-09 \backslash R M S F=2.542 e-06 \backslash D i p o l e=2.0219047,-1.034481$ $7,-1.1455193 \backslash \mathrm{PG}=\mathrm{C} 01 \quad[\mathrm{X}(\mathrm{C} 18 \mathrm{H} 18 \mathrm{~N} 105)] \backslash \backslash \mathrm{e}$

\section{5,6-dicarboxy-TMAO-}

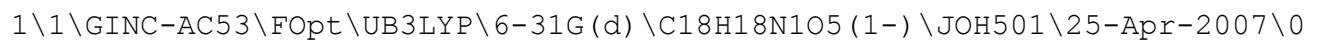

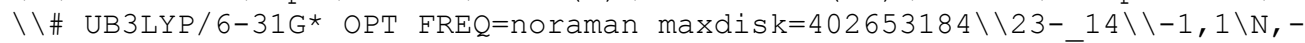
$0.0052667412,0.0159796244,-0.0090428408 \backslash 0,1.3603626477,0 . \overline{0} 098008971,-0$ 
$.0060533849 \backslash \mathrm{C},-2.7531740018,0.6878374196,-0.0040075871 \backslash \mathrm{C},-4.1944378809$ $, 0.8370689035,-0.0128522841 \backslash \mathrm{C},-4.8860030731,0.8404056475,1.235704542 \backslash \mathrm{C}$ $,-4.8762288314,1.018188296,-1.2461264098 \backslash \mathrm{C},-4.1929527423,0.7222679364$, $2.4140192939 \backslash \mathrm{C},-4.1580955258,1.0228745847,-2.4548436477 \backslash \mathrm{H},-4.731728897$ $5,0.7279335212,3.3598485059 \backslash \mathrm{C},-4.861629782,1.069372447,-3.7405782871 \backslash \mathrm{C}$ $,-2.7877049254,0.5868073998,2.4171949761 \backslash C,-2.748341405,0.8753072767,-$ $2.421959628 \backslash \mathrm{H},-2.2694836718,0.5002608429,3.3667260995 \backslash \mathrm{H},-2.2197694852$, $0.8758295379,-3.3665416592 \backslash \mathrm{C},-2.0627888363,0.5574673531,1.236480258 \backslash \mathrm{C}$, $-2.0448509692,0.7210891695,-1.2477781912 \backslash C,-0.5356146335,0.5951688605$, $-1.2697889289 \backslash \mathrm{C},-0.5438913849,0.4592851627,1.2981337104 \backslash \mathrm{C}, 0.108104792$, $1.9778631778,-1.5602978441 \backslash \mathrm{C}, 0.07405396,1.8070752578,1.7728201475 \backslash \mathrm{C},-0$ $.0835594792,-0.4085147206,-2.3586092451 \backslash C,-0.0970446704,-0.6499969435$, $2.283891615 \backslash \mathrm{H},-0.6206202696,-1.3561344959,-2.2435890733 \backslash \mathrm{H},-0.611952343$ $5,-1.588949539,2.0533378921 \backslash \mathrm{H},-0.2438168378,-0.0280700461,-3.373458009$ $3 \backslash \mathrm{H},-0.2863891437,-0.3860473552,3.3307712349 \backslash \mathrm{H}, 0.9807581439,-0.5864886$ $686,-2.1890660473 \backslash \mathrm{H}, 0.9742611187,-0.7917283158,2.1266715231 \backslash \mathrm{H},-0.35629$ $58199,2.7759145021,-0.9721130223 \backslash \mathrm{H},-0.3792379053,2.6634509004,1.262765$ $3306 \backslash \mathrm{H}, 1.160879385,1.8736590886,-1.2699752838 \backslash \mathrm{H}, 1.1392469481,1.7605467$ $736,1.5255295533 \backslash \mathrm{H}, 0.0299784021,2.2510223956,-2.6193810982 \backslash \mathrm{H},-0.050763$ $1843,1.955233644,2.8523478277 \backslash 0,-6.063761574,0.9218870097,-3.925779024$ $\backslash 0,-4.0306211706,1.2757138408,-4.8156090269 \backslash \mathrm{H},-4.6305886095,1.23400340$ $06,-5.5813753624 \backslash \mathrm{C},-6.3468428624,1.2851484131,-1.2251994384 \backslash \mathrm{H},-5.96636$ $56588,0.9240668758,1.2421303563 \backslash 0,-7.2112733988,0.5505263267,-0.786595$ $3812 \backslash 0,-6.6464384703,2.5353037569,-1.6748301439 \backslash \mathrm{H},-7.6213412119,2.5655$ $498407,-1.6873720172 \backslash \backslash$ Version=IA64L-G03RevD.01 \State $=1-A \backslash H F=-1127.6168$ $325 \backslash \mathrm{S} 2=0 . \backslash \mathrm{S} 2-1=0 . \backslash \mathrm{S} 2 \mathrm{~A}=0 . \backslash \mathrm{RMSD}=3.156 \mathrm{e}-10 \backslash \mathrm{RMSF}=5.870 \mathrm{e}-06 \backslash \mathrm{Thermal}=0 . \backslash \mathrm{Dipo}$ $l e=-1.8433463,1.1343337,-0.327745 \backslash P G=C 01 \quad[X(C 18 H 18 N 105)] \backslash \backslash 0$

\section{5,7-dicarboxy-TMAO• (46)}

$1 \backslash 1 \backslash G I N C-A C 21 \backslash F O p t \backslash U B 3 L Y P \backslash 6-31 G(d) \backslash C 18 H 18 N 105(2) \backslash J O H 501 \backslash 01-J u n-2006 \backslash 0 \backslash$

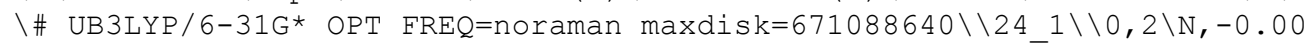
$69571109,-0.0103004336,-0.0106884995 \backslash 0,1.2771533887,-0 . \overline{0} 220581166,-0.0$ $085175012 \backslash \mathrm{C},-2.864965591,0.0592049615,0.0029588514 \backslash \mathrm{C},-4.3095355433,-0$. $0240508673,-0.0156597216 \backslash \mathrm{C},-5.00873694,-0.2951473399,1.212513115 \backslash \mathrm{C},-4$. $9890143088,0.1794201442,-1.243577353 \backslash C,-6.49348298,-0.4129733986,1.286$ $8833526 \backslash \mathrm{C},-4.2831733631,-0.4520641702,2.3807309384 \backslash \mathrm{C},-4.2836960973,0.4$ $622430066,-2.3951833856 \backslash \mathrm{H},-4.8047169957,-0.6484329931,3.3090892765 \backslash \mathrm{C},-$ $4.9609081139,0.6950556577,-3.7003713903 \backslash \mathrm{C},-2.8841135092,-0.3668930055$, $2.3873378142 \backslash \mathrm{C},-2.8756399342,0.5432370596,-2.3728475896 \backslash \mathrm{H},-2.367399800$ $1,-0.495911519,3.3322392045 \backslash \mathrm{H},-2.376131937,0.7727122304,-3.3066472871 \backslash$ $C,-2.1633071907,-0.1281970361,1.2317223974 \backslash C,-2.1628143971,0.340914501$ $5,-1.2112840766 \backslash \mathrm{C},-0.6439115732,0.4739441525,-1.2686709225 \backslash \mathrm{C},-0.645944$ $354,-0.0408870504,1.3345882774 \backslash C,-0.2432996067,1.9556377208,-1.4777079$ $262 \backslash C,-0.2362993016,1.2424728052,2.1003380732 \backslash C,-0.0551763083,-0.39429$ $50232,-2.4031894021 \backslash \mathrm{C},-0.0708680937,-1.2856123466,2.0494923915 \backslash \mathrm{H},-0.37$ $31360876,-1.4358242828,-2.2944846097 \backslash \mathrm{H},-0.4088255646,-2.20283219,1.557$ $3980524 \backslash \mathrm{H},-0.381164397,-0.0318341059,-3.3809809311 \backslash \mathrm{H},-0.3828212446,-1$. $3163682368,3.0964474512 \backslash \mathrm{H}, 1.0340797411,-0.3527321817,-2.3571048977 \backslash \mathrm{H}, 1$ $.0185640637,-1.2452953609,2.0078979799 \backslash \mathrm{H},-0.6849191413,2.5982651395,-0$ $.7094487961 \backslash \mathrm{H},-0.6715545598,2.134921616,1.6402747433 \backslash \mathrm{H}, 0.8455349389,2$. $0433178413,-1.4308841954 \backslash \mathrm{H}, 0.8531290152,1.3315182733,2.0867702711 \backslash \mathrm{H},-0$ $.5888634384,2.3101172185,-2.4534978314 \backslash \mathrm{H},-0.5784210831,1.1952756001,3$. $1386507733 \backslash 0,-4.3840122449,0.9539961792,-4.7388780421 \backslash 0,-6.3097224798$, $0.5922341327,-3.6301521055 \backslash \mathrm{H},-6.6360081234,0.762483405,-4.5330356232 \backslash \mathrm{H}$ $,-6.0663465748,0.1149894373,-1.2654666888 \backslash 0,-7.2895186106,-0.333819020$ $2,0.3697646272 \backslash 0,-6.932192812,-0.6362950617,2.5567455341 \backslash \mathrm{H},-7.90205173$ $57,-0.6992373426,2.4809056516 \backslash \backslash$ Version=IA64L-G03RevD. $01 \backslash$ State $=2-A \backslash H F=-$ $1127.6112154 \backslash \mathrm{S} 2=0.753734 \backslash \mathrm{S} 2-1=0 . \backslash \mathrm{S} 2 \mathrm{~A}=0.75001 \backslash \mathrm{RMSD}=2.896 \mathrm{e}-09 \backslash \mathrm{RMSF}=3.340$ e-06\Thermal $=0 . \backslash$ Dipole $=-0.563934,0.0240379,1.1684104 \backslash P G=C 01 \quad[X(C 18 H 18 \mathrm{~N}$ $105)] \backslash \backslash 0$

\section{5,7-dicarboxy-TMAO+}

$1 \backslash 1 \backslash G I N C-L C 56 \backslash$ FOpt \UB3LYP \6-31G(d) \C18H18N105 (1+) \JOH501\03-Jun-2006\0 


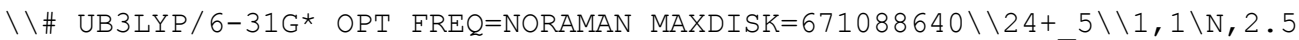
$447212842,-1.4138298739,-0.2532096594 \backslash 0,3.7447686181,-1.3 \overline{2} 76725003,-0$. $2635695298 \backslash \mathrm{C}, 0.0074729231,-0.2247145815,-0.280677877 \backslash \mathrm{C},-1.1826522044,0$ $.5783511845,-0.3038770172 \backslash \mathrm{C},-1.431849377,1.4317572933,0.8067303232 \backslash \mathrm{C},-$ $2.0489144004,0.480229776,-1.4454742769 \backslash C,-0.5599829926,1.4722372654,1$. $8795867371 \backslash C,-1.7014398041,-0.3563513144,-2.4937343684 \backslash \mathrm{C}, 0.5616153538$, $0.6163994126,1.936122591 \backslash C,-0.4795754553,-1.052129112,-2.502934336 \backslash C, 0$ $.8107886736,-0.259339974,0.9009149617 \backslash C, 0.3896471246,-0.9663374341,-1$. $43087768 \backslash \mathrm{C}, 1.8032223188,-1.4173724838,1.0718196393 \backslash \mathrm{C}, 1.8195356726,-1.4$ $645296551,-1.5867697594 \backslash \mathrm{C}, 1.0664429683,-2.7690634935,1.2215586567 \backslash \mathrm{C}, 1$. $9373138747,-2.9420194554,-2.0585034023 \backslash C, 2.8272827954,-1.2328265465,2$. $1883915344 \backslash \mathrm{C}, 2.6004104618,-0.5400178885,-2.5518284123 \backslash \mathrm{H}, 3.3531527283,-$ $0.2775355725,2.1149566316 \backslash \mathrm{H}, 2.5938692704,0.4924311236,-2.1912470255 \backslash \mathrm{H}$, $2.3088371677,-1.2750348686,3.1486061239 \backslash \mathrm{H}, 2.1045515457,-0.5665606402,-$ $3.5238400774 \backslash \mathrm{H}, 3.5652705764,-2.0382551972,2.1719806776 \backslash \mathrm{H}, 3.6316748691$, $-0.8758092034,-2.6729175193 \backslash \mathrm{H}, 0.3102615746,-2.9248952303,0.4511029382 \backslash$ $\mathrm{H}, 1.373466534,-3.6315140402,-1.4284964606 \backslash \mathrm{H}, 1.7730820448,-3.6049120826$ $, 1.2257366775 \backslash \mathrm{H}, 2.9887884814,-3.2405403848,-2.0828428217 \backslash \mathrm{H}, 0.555008950$ $2,-2.745453284,2.1883872609 \backslash \mathrm{H}, 1.5438991103,-3.0036574485,-3.0757352051$ $\backslash \mathrm{C},-0.7753336191,2.3718077787,3.0579722355 \backslash \mathrm{H},-2.3100560167,2.062039619$ $, 0.7964289664 \backslash \mathrm{C},-3.3291634763,1.2614769449,-1.5453022833 \backslash \mathrm{H},-2.36410453$ $68,-0.4431129448,-3.3454942683 \backslash \mathrm{H},-0.2164457645,-1.6211330363,-3.389189$ $377 \backslash \mathrm{H}, 1.163678126,0.6429309748,2.8364965542 \backslash 0,-0.0379807731,2.39181375$ $73,4.0203775252 \backslash 0,-1.8656781777,3.1472454231,2.9263072329 \backslash \mathrm{H},-1.9285623$ $208,3.6975261419,3.7303557323 \backslash 0,-3.7038823294,2.1156893219,-0.76995082$ $97 \backslash 0,-4.0491442519,0.9060187952,-2.6300084908 \backslash \mathrm{H},-4.8530110434,1.460190$ $5141,-2.6257720506 \backslash \backslash$ Version=IA32L-G03RevC.02 $\backslash$ State $=1-A \backslash H F=-1127.359184$ $5 \backslash S 2=0 . \backslash S 2-1=0 . \backslash S 2 A=0 . \backslash R M S D=6.209 e-09 \backslash R M S F=2.495 e-06 \backslash D i p o l e=1.3676574$, $-2.1435596,-1.3841796 \backslash \mathrm{PG}=\mathrm{C} 01 \quad[\mathrm{X}(\mathrm{C} 18 \mathrm{H} 18 \mathrm{~N} 105)] \backslash \backslash @$

\section{5,7-dicarboxy-TMAO-}

$1 \backslash 1 \backslash$ GINC-AC51 5 FOpt \UB3LYP \6-31G (d) \C18H18N105 (1-) \JOH501 $006-$ Dec-2006 0

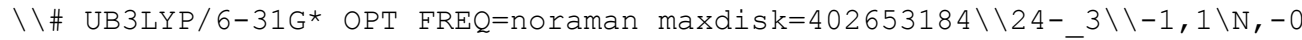
$.0531037118,0.2277689562,-0.0326358629 \backslash 0,1.2961513234,0.0 \overline{8} 76852653,-0$. $0050540829 \backslash \mathrm{C},-2.8684522203,-0.0034710098,0.0034912305 \backslash \mathrm{C},-4.3203610751$, $0.0797826784,-0.0037840384 \backslash C,-5.0137142553,-0.0083333971,-1.2773193017$ $\backslash C,-4.9987722791,0.222688951,1.2201590238 \backslash C,-6.4726248914,0.0828642877$ $,-1.401795436 \backslash C,-4.2624983244,-0.1991668752,-2.4336601597 \backslash C,-4.2998561$ $957,0.2719439386,2.427332931 \backslash \mathrm{H},-4.7759317402,-0.2779199441,-3.38524198$ $14 \backslash \mathrm{C},-4.9804008651,0.4040474072,3.7204262631 \backslash \mathrm{C},-2.8707724894,-0.291450$ $3267,-2.402462864 \backslash \mathrm{C},-2.8887213762,0.1922953968,2.4264565776 \backslash \mathrm{H},-2.33982$ $92469,-0.4490485068,-3.335016713 \backslash \mathrm{H},-2.3810485178,0.2369196806,3.383179$ $0303 \backslash C,-2.1520121921,-0.1796424852,-1.2121800689 \backslash \mathrm{C},-2.1742384816,0.059$ $5720919,1.2563762826 \backslash C,-0.6610368208,-0.0318127817,1.2943235836 \backslash C,-0.6$ $382309789,-0.3454847485,-1.260339864 \backslash C,-0.2216155678,-1.4174962971,1.8$ $385480487 \backslash \mathrm{C},-0.2451794089,-1.8381668054,-1.4927861029 \backslash \mathrm{C},-0.0736640361$, $1.0744986587,2.2047866899 \backslash \mathrm{C},-0.018582605,0.4885960028,-2.4123204318 \backslash \mathrm{H}$, $-0.4647189047,2.0530500511,1.9065503113 \backslash \mathrm{H},-0.3614623656,1.5271917064,-$ $2.3514468169 \backslash \mathrm{H},-0.3045398306,0.9076633935,3.2619480238 \backslash \mathrm{H},-0.2667688063$ $, 0.0877848552,-3.4014122272 \backslash \mathrm{H}, 1.0074964365,1.0685185574,2.0518847595 \backslash \mathrm{H}$ $, 1.0632849951,0.4706305332,-2.2703823856 \backslash \mathrm{H},-0.7758924998,-2.2336967133$ $, 1.3645015957 \backslash \mathrm{H},-0.8040564864,-2.5101562326,-0.8334255442 \backslash \mathrm{H}, 0.84367959$ $55,-1.5129089383,1.5984347166 \backslash \mathrm{H}, 0.8224511408,-1.9278533549,-1.27470565$ $94 \backslash \mathrm{H},-0.373838163,-1.4924911557,2.9216417358 \backslash \mathrm{H},-0.4343088884,-2.149377$ $2454,-2.5272752102 \backslash 0,-4.4426187127,0.4426745813,4.8205062782 \backslash 0,-6.3460$ $795849,0.4883186799,3.6251978423 \backslash \mathrm{H},-6.6394136202,0.5708065359,4.550003$ $2356 \backslash \mathrm{H},-6.0765119158,0.2867947049,1.2172034836 \backslash 0,-7.3078694222,0.24336$ $68673,-0.5191202126 \backslash 0,-6.9187639902,-0.0298729649,-2.7045257259 \backslash \mathrm{H},-7.8$ $843830205,0.0520560013,-2.612070954 \backslash \backslash$ Version=IA64L-G03RevD.01 \State=1$\mathrm{A} \backslash \mathrm{HF}=-1127.6304229 \backslash \mathrm{S} 2=0 . \backslash \mathrm{S} 2-1=0 . \backslash \mathrm{S} 2 \mathrm{~A}=0 . \backslash \mathrm{RMSD}=6.738 \mathrm{e}-09 \backslash \mathrm{RMSF}=7.694 \mathrm{e}-06 \backslash$ Thermal $=0 . \backslash$ Dipole $=-2.0971253,-0.3953408,-0.8164681 \backslash P G=C 01 \quad[X(C 18 H 18 \mathrm{~N} 10$ 5) $] \backslash \backslash 0$ 


\section{5,8-dicarboxy-TMAO• (47)}

$1 \backslash 1 \backslash G I N C-L C 136 \backslash F O p t \backslash U B 3 L Y P \backslash 6-31 G(d) \backslash C 18 H 18 N 105$ (2) \JOH501\09-Jun-2006\0

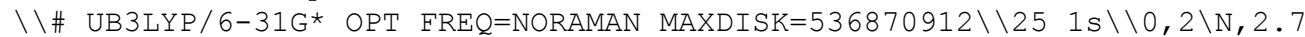
$621520178,0.0816556818,0 . \backslash 0,4.0462229502,0.0800668531,0 .{ }_{C},-0.07774294$ $28,0.0350681852,0 . \backslash \mathrm{C},-1.5147530051,0.1272157626,0 . \backslash \mathrm{C},-2.2201426105,0.1$ $662454427,1.2264824233 \backslash \mathrm{C},-2.2201426105,0.1662454427,-1.2264824233 \backslash \mathrm{H},-3$ $.3024842722,0.2379100846,1.2145259918 \backslash \mathrm{H},-3.3024842722,0.2379100846,-1$. $2145259918 \backslash \mathrm{C},-1.5357280087,0.105460256,2.4220482136 \backslash \mathrm{C},-1.5357280087,0$. $105460256,-2.4220482136 \backslash \mathrm{C},-2.2240251127,0.1310399313,3.739180665 \backslash \mathrm{C},-2$. $2240251127,0.1310399313,-3.739180665 \backslash 0,-3.5736237041,0.2292874062,3.63$ $33672471 \backslash 0,-3.5736237041,0.2292874062,-3.6333672471 \backslash \mathrm{H},-3.9102885003,0$. $23710322,4.5484275381 \backslash \mathrm{H},-3.9102885003,0.23710322,-4.5484275381 \backslash 0,-1.66$ $23778163,0.0720344866,4.8149581776 \backslash 0,-1.6623778163,0.0720344866,-4.814$ $9581776 \backslash \mathrm{C},-0.1230586769,0.0135870199,2.4205403786 \backslash \mathrm{C},-0.1230586769,0.01$ $35870199,-2.4205403786 \backslash \mathrm{H}, 0.366930505,-0.0352754262,3.3861759768 \backslash \mathrm{H}, 0.36$ $6930505,-0.0352754262,-3.3861759768 \backslash C, 0.6083694157,-0.0136975952,1.250$ $5858604 \backslash \mathrm{C}, 0.6083694157,-0.0136975952,-1.2505858604 \backslash \mathrm{C}, 2.1254508342,-0.1$ $336736365,1.333741225 \backslash \mathrm{C}, 2.1254508342,-0.1336736365,-1.333741225 \backslash \mathrm{C}, 2.71$ $21911499,0.9493302664,2.2664430009 \backslash C, 2.7121911499,0.9493302664,-2.2664$ $430009 \backslash \mathrm{H}, 2.3975273503,1.9464865714,1.9431349636 \backslash \mathrm{H}, 2.3975273503,1.94648$ $65714,-1.9431349636 \backslash \mathrm{H}, 2.3798677079,0.7967733961,3.2964457859 \backslash \mathrm{H}, 2.37986$ $77079,0.7967733961,-3.2964457859 \backslash \mathrm{H}, 3.8015696832,0.897190831,2.23494184$ $87 \backslash \mathrm{H}, 3.8015696832,0.897190831,-2.2349418487 \backslash \mathrm{C}, 2.524550088,-1.540685973$ $4,1.8430674501 \backslash \mathrm{C}, 2.524550088,-1.5406859734,-1.8430674501 \backslash \mathrm{H}, 2.084745660$ $8,-2.3272383406,1.2215548101 \backslash \mathrm{H}, 2.0847456608,-2.3272383406,-1.221554810$ $1 \backslash \mathrm{H}, 2.1762829595,-1.6866378732,2.870211393 \backslash \mathrm{H}, 2.1762829595,-1.686637873$ $2,-2.870211393 \backslash \mathrm{H}, 3.6132825757,-1.6354360144,1.8171557361 \backslash \mathrm{H}, 3.613282575$ $7,-1.6354360144,-1.8171557361 \backslash \backslash$ Version=IA32L-G03RevC.02 $\backslash$ State $=2-A^{\prime} \backslash H F=$ $-1127.6169454 \backslash S 2=0.753746 \backslash S 2-1=0 . \backslash S 2 A=0.75001 \backslash R M S D=7.263 e-09 \backslash R M S F=2.07$ $2 e-05 \backslash$ Dipole $=-1.3357244,-0.1448513,0 . \backslash P G=C S \quad[S G(C 2 N 1 O 1), X(C 16 H 1804)] \backslash \backslash$ a

\section{5,8-dicarboxy-TMAO+}

$1 \backslash 1 \backslash G I N C-L C 52 \backslash F O p t \backslash U B 3 L Y P \backslash 6-31 G(d) \backslash C 18 H 18 N 105(1+) \backslash J O H 501 \backslash 03-J u n-2006 \backslash 0$ \\\# UB3LYP/6-31G* OPT FREQ=NORAMAN MAXDISK=671088640 \\25+ $1 \backslash \backslash 1,1 \backslash \mathrm{N}, 2.2$ $505116287,-1.5248647912,0.0621703679 \backslash 0,3.4435031059,-1.65 \overline{3} 1620266,0.13$ $34163485 \backslash C,-0.0729152411,0.028134612,-0.0239787333 \backslash C,-1.1343565904,0.9$ $882468234,-0.0441760324 \backslash C,-2.0360983912,1.0473716191,1.0485169179 \backslash C,-1$ $.2532476799,1.8589901793,-1.1564491062 \backslash C,-1.8780407116,0.2007140064,2$. $1281781033 \backslash \mathrm{C},-0.3619998207,1.7779324892,-2.210322912 \backslash \mathrm{C},-0.7720599468,-$ $0.6801708557,2.1837417204 \backslash C, 0.6385604781,0.7771282468,-2.2235799923 \backslash \mathrm{C}$, $0.1382143733,-0.7494862099,1.1474161846 \backslash \mathrm{C}, 0.7515615497,-0.1173561022,-$ $1.1779526692 \backslash \mathrm{C}, 1.4594034103,-1.4729218426,1.3606800721 \backslash \mathrm{C}, 1.597673426,-$ $1.3885758067,-1.3079719049 \backslash \mathrm{C}, 1.3097263571,-2.9561666637,1.8072290322 \backslash \mathrm{C}$ $, 0.7010898299,-2.6307820465,-1.5330881153 \backslash \mathrm{C}, 2.3368587206,-0.7046528046$ $, 2.3787803168 \backslash \mathrm{C}, 2.7060586187,-1.3242079735,-2.3553896727 \backslash \mathrm{H}, 2.514168413$ $5,0.3210335596,2.0431738345 \backslash \mathrm{H}, 3.3509764725,-0.4518272022,-2.2209012768$ $\backslash \mathrm{H}, 1.7998082054,-0.6663268043,3.3283591766 \backslash \mathrm{H}, 2.2477127696,-1.271927942$ $2,-3.3452053125 \backslash \mathrm{H}, 3.2928932035,-1.206844759,2.5356124935 \backslash \mathrm{H}, 3.324760542$ $3,-2.2237199507,-2.3229226464 \backslash \mathrm{H}, 0.6819389592,-3.5373842926,1.130426220$ $4 \backslash \mathrm{H},-0.1180071928,-2.6961023956,-0.8153623845 \backslash \mathrm{H}, 2.2957967253,-3.421161$ $8882,1.8896129597 \backslash \mathrm{H}, 1.2916075179,-3.5519606505,-1.5096168991 \backslash \mathrm{H}, 0.84525$ $57058,-2.9606733152,2.7959988146 \backslash \mathrm{H}, 0.2627733452,-2.527242369,-2.530111$ $2131 \backslash \mathrm{C},-2.8057991016,0.1950235229,3.3014239482 \backslash \mathrm{H},-2.8537537089,1.76005$ $47768,1.029221333 \backslash \mathrm{C},-0.4259244809,2.6830941436,-3.4001421616 \backslash \mathrm{H}, 1.26384$ $43574,0.7188096952,-3.1067226122 \backslash \mathrm{H},-0.6558916289,-1.2626808359,3.09217$ $16539 \backslash 0,-2.655949311,-0.5237184175,4.2659151887 \backslash 0,-3.8189132256,1.0713$ $242687,3.1644080436 \backslash \mathrm{H},-4.3701217243,1.002922679,3.9674315075 \backslash \mathrm{H},-2.0468$ $465595,2.5986008163,-1.172325211 \backslash 0,0.3298403046,2.5979517354,-4.343571$ $401 \backslash 0,-1.4100594387,3.5969293705,-3.3014752083 \backslash \mathrm{H},-1.3800970851,4.14199$ $2948,-4.1110667579 \backslash \backslash$ Version=IA32L-G03RevC.02 State=1-A \HF=-1127.364771 $\backslash S 2=0 . \backslash S 2-1=0 . \backslash S 2 A=0 . \backslash R M S D=8.825 e-09 \backslash R M S F=5.964 e-06 \backslash D i p o l e=0.831055,-1$ $.1608445,0.0211492 \backslash \mathrm{PG}=\mathrm{C} 01 \quad[\mathrm{X}(\mathrm{C} 18 \mathrm{H} 18 \mathrm{~N} 105)] \backslash \backslash @$ 


\section{5,8-dicarboxy-TMAO-}

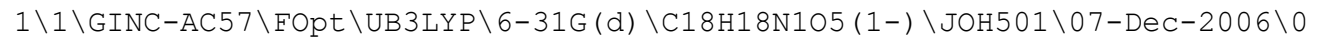

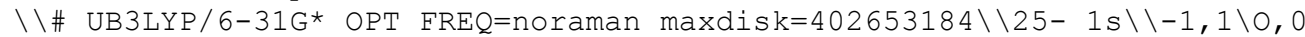
$.4 .0305367078,-0.3519999906 \backslash \mathrm{N}, 0 ., 2.6681156881,-0.4673147 \overline{3} 04 \backslash \mathrm{C}, 0 .,-0.1$ $084752537,-0.0773146335 \backslash C, 0 .,-1.5573150063,-0.0718244159 \backslash C, 1.288923653$ $3,2.0964660651,-0.005416891 \backslash \mathrm{C},-1.2889236533,2.0964660651,-0.005416891 \backslash$ $C, 1.2507638625,0.5797530276,-0.0486182343 \backslash C,-1.2507638625,0.5797530276$ $,-0.0486182343 \backslash C, 2.3275104093,2.6480331264,-1.0138212097 \backslash C,-2.32751040$ $93,2.6480331264,-1.0138212097 \backslash C,-1.2258999955,-2.2572454155,-0.0558648$ $433 \backslash \mathrm{C}, 1.2258999955,-2.2572454155,-0.0558648433 \backslash \mathrm{H},-1.2303230152,-3.3434$ $84783,-0.0561267283 \backslash \mathrm{H}, 1.2303230152,-3.343484783,-0.0561267283 \backslash \mathrm{C},-2.428$ $458042,-1.5736243153,-0.0229818909 \backslash C, 2.428458042,-1.5736243153,-0.0229$ $818909 \backslash \mathrm{C},-3.6716262387,-2.3582191402,0.0086541949 \backslash \mathrm{C}, 3.6716262387,-2.35$ $82191402,0.0086541949 \backslash 0,-4.8039855811,-1.5872279193,0.0568049016 \backslash 0,4.8$ $039855811,-1.5872279193,0.0568049016 \backslash \mathrm{H},-5.5313750091,-2.2347829913,0.0$ $712040027 \backslash \mathrm{H}, 5.5313750091,-2.2347829913,0.0712040027 \backslash 0,-3.7607861864,-3$ $.5783805846,-0.0048696466 \backslash 0,3.7607861864,-3.5783805846,-0.0048696466 \backslash \mathrm{C}$ $,-2.4239744583,-0.1454494665,-0.0179014765 \backslash C, 2.4239744583,-0.145449466$ $5,-0.0179014765 \backslash \mathrm{H},-3.3751685263,0.3708710848,0.0118914126 \backslash \mathrm{H}, 3.37516852$ $63,0.3708710848,0.0118914126 \backslash \mathrm{H},-2.1518411273,2.2206263277,-2.007034314$ $8 \backslash \mathrm{H}, 2.1518411273,2.2206263277,-2.0070343148 \backslash \mathrm{H},-3.359496917,2.437345866$ $6,-0.7113952114 \backslash \mathrm{H}, 3.359496917,2.4373458666,-0.7113952114 \backslash \mathrm{H},-2.16512711$ $87,3.7267654394,-1.0650197313 \backslash \mathrm{H}, 2.1651271187,3.7267654394,-1.065019731$ $3 \backslash C,-1.6696967656,2.5932252488,1.4174833232 \backslash C, 1.6696967656,2.593225248$ $8,1.4174833232 \backslash \mathrm{H},-1.1305939437,2.0456438713,2.1976929764 \backslash \mathrm{H}, 1.130593943$ $7,2.0456438713,2.1976929764 \backslash \mathrm{H},-2.7435688935,2.4926051553,1.6155521889 \backslash$ $\mathrm{H}, 2.7435688935,2.4926051553,1.6155521889 \backslash \mathrm{H},-1.3813861488,3.6501133347$, $1.448234063 \backslash \mathrm{H}, 1.3813861488,3.6501133347,1.448234063 \backslash \backslash$ Version=IA64L-G03 RevD. $01 \backslash$ State $=1-A^{\prime} \backslash H F=-1127.6343043 \backslash S 2=0 . \backslash S 2-1=0 . \backslash S 2 A=0 . \backslash R M S D=5.105 e-0$ $9 \backslash \mathrm{RMSF}=8.925 \mathrm{e}-06 \backslash \mathrm{Thermal}=0 . \backslash \mathrm{Dipole}=0 .,-1.5411565,0.4402046 \backslash \mathrm{PG}=\mathrm{CS} \quad[\mathrm{SG}(\mathrm{C}$ $2 \mathrm{~N} 101), \mathrm{X}(\mathrm{C} 16 \mathrm{H} 1804)] \backslash \backslash \mathrm{Q}$

\section{6,7-dicarboxy-TMAO• (48)}

$1 \backslash 1 \backslash$ GINC-LC116\FOpt \UB3LYP \6-31G (d) \C18H18N1O5 (2) \JOH501 \25-May-2006\0

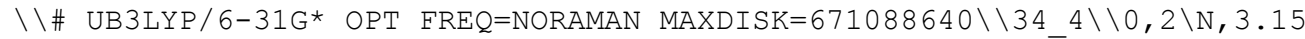
$82911725,-0.0681503791,0.0623235092 \backslash 0,4.4413865183,-0.11 \overline{5} 7984562,0.088$ $5642059 \backslash \mathrm{C}, 0.2971704482,-0.010001314,0.0074222535 \backslash \mathrm{C},-1.1401902302,0.134$ $6221202,-0.0404069096 \backslash \mathrm{C},-1.8183767183,0.5894898667,1.1366872599 \backslash \mathrm{C},-1.8$ $184026126,-0.1934687944,-1.2578838049 \backslash \mathrm{C},-3.1952761196,1.1627665536,1.0$ $956918953 \backslash \mathrm{C},-3.2848944575,-0.4657040516,-1.3100653929 \backslash \mathrm{C},-1.1253367369$, $0.6938063685,2.326809936 \backslash \mathrm{C},-1.085651052,-0.4777597996,-2.3943597251 \backslash \mathrm{H}$, $-1.6070668736,-0.7159061102,-3.3146429164 \backslash C, 0.2541009705,0.4439528627$, $2.388689546 \backslash \mathrm{C}, 0.3159083128,-0.5149642842,-2.3640947744 \backslash \mathrm{H}, 0.8461260937$, $-0.7478484323,-3.2813253421 \backslash \mathrm{C}, 0.9821188842,0.1493404025,1.2530466861 \backslash \mathrm{C}$ $, 1.015306917,-0.3225360087,-1.1884592742 \backslash \mathrm{C}, 2.4868960059,-0.0534854426$, $1.3920261284 \backslash \mathrm{C}, 2.5313592962,-0.4764191142,-1.2254496563 \backslash \mathrm{C}, 2.7757314243$ $,-1.401181693,2.1007845115 \backslash \mathrm{C}, 2.9171116795,-1.950244236,-1.5073110757 \backslash \mathrm{C}$ $, 3.1372708776,1.1048764384,2.1817910765 \backslash C, 3.1454391552,0.4443127177,-2$ $.3056754251 \backslash \mathrm{H}, 2.8854916799,2.0686394791,1.7286130221 \backslash \mathrm{H}, 2.8355713911,1$. $481875063,-2.1473976278 \backslash \mathrm{H}, 2.7991503747,1.1097170785,3.2208097386 \backslash \mathrm{H}, 2.8$ $321957924,0.1370458069,-3.3066610068 \backslash \mathrm{H}, 4.2209267106,0.9815788893,2.166$ $2310684 \backslash \mathrm{H}, 4.2334968177,0.3883068865,-2.2436755685 \backslash \mathrm{H}, 2.2878042658,-2.23$ $37080551,1.5844445639 \backslash \mathrm{H}, 2.4563978549,-2.6263386814,-0.7804302124 \backslash \mathrm{H}, 3.8$ $553904356,-1.5740838942,2.1129943571 \backslash \mathrm{H}, 4.0037102281,-2.0524761226,-1.4$ $483003052 \backslash \mathrm{H}, 2.4052001376,-1.3790069078,3.1303113543 \backslash \mathrm{H}, 2.5837322114,-2$. $249245317,-2.5054257777 \backslash \mathrm{H},-1.6501890878,1.0177112448,3.2185027315 \backslash 0,-3$ $.6576662352,1.8037249629,0.1733543528 \backslash 0,-3.8657982235,0.9925038124,2.2$ $604247945 \backslash \mathrm{H},-4.7147685139,1.4579394362,2.1411671433 \backslash \mathrm{H}, 0.7522230484,0.5$ $345154717,3.3475887935 \backslash 0,-3.9313653446,-0.9857663396,-0.4229841192 \backslash 0,-$ $3.8259701157,-0.1719803475,-2.516656317 \backslash \mathrm{H},-4.7598398335,-0.4475477915$, $-2.4561514454 \backslash \backslash$ Version=IA32L-G03RevC.02 \State $=2-A \backslash H F=-1127.5960251 \backslash \mathrm{S} 2=$ $0.75374 \backslash \mathrm{S} 2-1=0 . \backslash \mathrm{S} 2 \mathrm{~A}=0.75001 \backslash \mathrm{RMSD}=4.363 \mathrm{e}-09 \backslash \mathrm{RMSF}=2.935 \mathrm{e}-06 \backslash \mathrm{Dipole}=-0.06$ $53892,-0.2609566,0.0444974 \backslash \mathrm{PG}=\mathrm{C} 01 \quad[\mathrm{X}(\mathrm{C} 18 \mathrm{H} 18 \mathrm{~N} 105)] \backslash \backslash \mathrm{Q}$ 


\section{6,7-dicarboxy-TMAO+}

$1 \backslash 1 \backslash G I N C-L C 105 \backslash F O p t \backslash U B 3 L Y P \backslash 6-31 G(d) \backslash C 18 H 18 N 105(1+) \backslash J O H 501 \backslash 28-M a y-2006 \backslash$

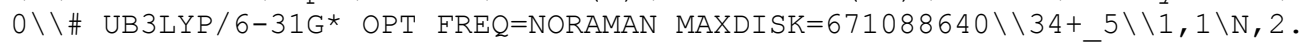
$5633187785,-1.7704057939,-0.0281014176 \backslash 0,3.76367768,-1.8594480784,-0.0$ $708973435 \backslash \mathrm{C}, 0.2146882484,-0.2387032301,-0.018404923 \backslash \mathrm{C},-0.8523344452,0$. $7168351288,-0.0191037993 \backslash \mathrm{C},-1.736274091,0.7314254747,1.1100458807 \backslash \mathrm{C},-0$ $.9516043299,1.5962847817,-1.1461511388 \backslash \mathrm{C},-3.0912567227,1.3738349739,1$. $0375073413 \backslash \mathrm{C},-1.7389821422,2.8750262638,-1.0806532638 \backslash \mathrm{C},-1.4386899714$, $-0.0268346148,2.2269461671 \backslash \mathrm{C},-0.149515054,1.3962543222,-2.2571899423 \backslash \mathrm{H}$ $,-2.1125382994,-0.005950116,3.0755251162 \backslash \mathrm{H},-0.2462037683,2.0650259893$, $-3.1048257419 \backslash \mathrm{C},-0.2783182634,-0.8214742002,2.2832339025 \backslash \mathrm{C}, 0.782765081$ $9,0.3442925783,-2.3119789699 \backslash \mathrm{H}, 1.3382979403,0.1855159087,-3.2286007563$ $\backslash \mathrm{C}, 0.5438407285,-0.9366197147,1.1793411829 \backslash \mathrm{C}, 0.9525263082,-0.480886722$ $8,-1.2169302108 \backslash C, 1.904395513,-1.6017397272,1.3272143914 \backslash C, 1.764713159$ $,-1.7788526991,-1.3177184543 \backslash C, 1.8549669417,-3.0343057088,1.9326945201$ $\backslash \mathrm{C}, 0.8417597242,-3.020707129,-1.321504664 \backslash \mathrm{C}, 2.8463027633,-0.7068958476$ $, 2.1699870825 \backslash \mathrm{C}, 2.7485341333,-1.8372301303,-2.4835570384 \backslash \mathrm{H}, 2.948298819$ $7,0.2831893313,1.7170338302 \backslash \mathrm{H}, 3.4055475693,-0.9643120187,-2.5159411841$ $\backslash \mathrm{H}, 2.4057501388,-0.5856420909,3.1612657646 \backslash \mathrm{H}, 2.1826587741,-1.889407705$ $8,-3.4162219911 \backslash \mathrm{H}, 3.8323460433,-1.1622991018,2.2758465487 \backslash \mathrm{H}, 3.36865687$ $44,-2.7342950225,-2.4208737956 \backslash \mathrm{H}, 1.1807613025,-3.6970914546,1.38819369$ $48 \backslash \mathrm{H}, 0.1171983709,-3.0131047751,-0.5063168426 \backslash \mathrm{H}, 2.8604395913,-3.463240$ $1096,1.9503729898 \backslash \mathrm{H}, 1.4283822826,-3.9441512365,-1.2901023002 \backslash \mathrm{H}, 1.50170$ $0648,-2.9532983183,2.963009315 \backslash \mathrm{H}, 0.28250197,-2.9986141431,-2.261541953$ $4 \backslash \mathrm{H},-0.0438662567,-1.3343286986,3.2107318686 \backslash 0,-3.7565776519,1.4191875$ $713,0.0255525859 \backslash 0,-3.5202350773,1.7953195422,2.239083005 \backslash \mathrm{H},-4.4239983$ $939,2.143761424,2.112463026 \backslash 0,-1.8200509485,3.5551999417,-0.0817197326$ $\backslash 0,-2.2404436928,3.2243270446,-2.2766168873 \backslash \mathrm{H},-2.6852330229,4.08617173$ $28,-2.1591890684 \backslash \backslash$ Version=IA32L-G03RevC.02 S State $=1-A \backslash H F=-1127.34414 \backslash \mathrm{S} 2$ $=0 . \backslash \mathrm{S} 2-1=0 . \backslash \mathrm{S} 2 \mathrm{~A}=0 . \backslash \mathrm{RMSD}=3.238 \mathrm{e}-09 \backslash \mathrm{RMSF}=5.686 \mathrm{e}-06 \backslash \mathrm{Dipole}=2.0294476,-2.2$ $453487,0.052206 \backslash P G=C 01 \quad[X(\mathrm{C} 18 \mathrm{H} 18 \mathrm{~N} 105)] \backslash \backslash @$

\section{6,7-dicarboxy-TMAO-}

$1 \backslash 1 \backslash G I N C-A C 57 \backslash F O p t \backslash U B 3 L Y P \backslash 6-31 G(d) \backslash C 18 H 18 N 105$ (1-) \JOH501 \07-Dec-2006\0

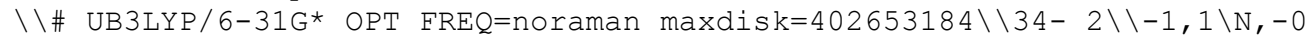
$.0902445572,-0.0873866914,-0.2849676206 \backslash 0,1.2693717304,-0.0590376314,-$ $0.0680949472 \backslash C,-0.6744747968,1.2622141269,-0.1214311531 \backslash C,-0.070069365$ $6,2.07484755,-1.2963950451 \backslash C,-2.9225188181,-0.0240209123,0.0073374967 \backslash$ $C,-4.3730187736,-0.0295751517,-0.0570928213 \backslash C,-5.0429221646,1.23952026$ $3,-0.1628654668 \backslash C,-5.0424544185,-1.2929788395,0.0025243208 \backslash C,-6.465733$ $7787,1.4999021802,0.1425122823 \backslash \mathrm{C},-6.4400556987,-1.5496778382,-0.408176$ $2255 \backslash \mathrm{C},-4.2976409371,2.3827879064,-0.4213989314 \backslash \mathrm{C},-4.3256083372,-2.439$ $7578782,0.3422006082 \backslash \mathrm{H},-4.8296460813,3.3238302152,-0.5254929847 \backslash \mathrm{H},-4.8$ $659697484,-3.3778886345,0.4176540041 \backslash C,-2.903708289,2.3523067325,-0.48$ $0667506 \backslash \mathrm{C},-2.9420295128,-2.4017165935,0.521667552 \backslash \mathrm{H},-2.3663732476,3.27$ $51291599,-0.6698261921 \backslash \mathrm{H},-2.4192096113,-3.3176531798,0.777085847 \backslash \mathrm{C},-2$. $1941298128,1.1823660005,-0.2273778691 \backslash \mathrm{C},-2.2210184255,-1.2340492278,0$. $3216793942 \backslash \mathrm{C},-0.7125921995,-1.2300504375,0.4343491166 \backslash \mathrm{C},-0.2552079581$, $1.9707997886,1.2049807538 \backslash \mathrm{C},-0.3043040025,-1.237666451,1.9311261316 \backslash \mathrm{C}$, $-0.1011570986,-2.4745653462,-0.2555777201 \backslash \mathrm{H},-0.4386311704,1.686905808$, $-2.2522653525 \backslash \mathrm{H},-0.4887754991,-2.570096483,-1.2755899513 \backslash \mathrm{H},-0.29232960$ $92,3.146628811,-1.2336431794 \backslash \mathrm{H},-0.3009794406,-3.4034154483,0.291021498$ $1 \backslash \mathrm{H}, 1.0094986536,1.9188629672,-1.2498747856 \backslash \mathrm{H}, 0.9742795195,-2.28960456$ $72,-0.3042523981 \backslash \mathrm{H},-0.828462047,1.6062950857,2.0636500798 \backslash \mathrm{H},-0.8912294$ $535,-0.5296997459,2.5244079106 \backslash \mathrm{H}, 0.8037160598,1.7414304285,1.352433120$ $8 \backslash \mathrm{H}, 0.7516586753,-0.9347069327,1.9303613067 \backslash \mathrm{H},-0.3960148905,3.05702422$ $24,1.1460932124 \backslash \mathrm{H},-0.4194520201,-2.2314019548,2.3814094668 \backslash 0,-7.138482$ $2896,-2.4755815628,-0.0285339834 \backslash 0,-7.1297700391,2.4322951368,-0.28221$ $17544 \backslash 0,-6.9930967084,0.6609855908,1.0859651644 \backslash 0,-6.887977781,-0.7178$ $285227,-1.3973751255 \backslash \mathrm{H},-7.912433884,0.9652618148,1.1941170955 \backslash \mathrm{H},-7.799$ $2621725,-1.0159037581,-1.5713753491 \backslash \backslash$ Version=IA64L-G03RevD.01 \State=1$\mathrm{A} \backslash \mathrm{HF}=-1127.6118703 \backslash \mathrm{S} 2=0 . \backslash \mathrm{S} 2-1=0 . \backslash \mathrm{S} 2 \mathrm{~A}=0 . \backslash \mathrm{RMSD}=3.393 \mathrm{e}-09 \backslash \mathrm{RMSF}=2.314 \mathrm{e}-06 \backslash$ Thermal $=0 . \backslash$ Dipole $=-2.610449,0.0651434,0.3001761 \backslash \mathrm{PG}=\mathrm{C} 01 \quad[\mathrm{X}(\mathrm{C} 18 \mathrm{H} 18 \mathrm{~N} 105)$ ] $\backslash \backslash Q$ 


\section{4-amino-TMAO• (49)}

$1 \backslash 1 \backslash G I N C-A C 54 \backslash F O p t \backslash U B 3 L Y P \backslash 6-31 G(d) \backslash C 16 H 19 N 201$ (2) \JOH501\15-Apr-2007\0

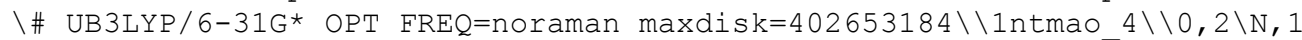
$.7641656178,-0.6987833472,-0.2663033302 \backslash 0,2.8898182374,-1.3091161678,-$ $0.3687200564 \backslash C, 0.5788171961,-1.5936857817,-0.0579150844 \backslash C, 0.6081664584$ $,-2.5498452112,-1.2774008098 \backslash C,-0.7280758956,0.6334287258,-0.034275885$ $4 \backslash \mathrm{C},-1.9717218355,1.3589535344,-0.0554203989 \backslash \mathrm{C},-1.9861612228,2.7739177$ $114,-0.0692740616 \backslash \mathrm{C},-3.188678687,0.6355354832,-0.0460964416 \backslash \mathrm{H},-2.94687$ $09054,3.2830626057,-0.0888555789 \backslash \mathrm{H},-4.1280195468,1.182209396,-0.070139$ $0691 \backslash \mathrm{C},-0.8109003239,3.4839720639,-0.0474169151 \backslash \mathrm{C},-3.1832067742,-0.727$ $6810988,0.0121949915 \backslash \mathrm{H},-0.8141672957,4.5701964445,-0.0507561279 \backslash \mathrm{H},-4.1$ $212004467,-1.278844174,0.0290024621 \backslash \mathrm{C}, 0.4123123042,2.7864854439,-0.017$ $1439097 \backslash \mathrm{C},-1.9669715693,-1.4670862182,0.0443393688 \backslash \mathrm{H}, 1.331099993,3.363$ $172281,0.0142277556 \backslash \mathrm{N},-2.1200449305,-2.8615892282,0.0645193704 \backslash \mathrm{C}, 0.478$ $2442637,1.4031109949,-0.0184775487 \backslash \mathrm{C},-0.7339217248,-0.8012839695,-0.01$ $11198473 \backslash C, 1.8500505419,0.7503683124,0.0636689363 \backslash C, 2.4316941554,0.891$ $9779126,1.4928772586 \backslash \mathrm{C}, 0.8540766507,-2.3587081663,1.2659614747 \backslash \mathrm{C}, 2.831$ $1078281,1.3610282185,-0.9619867758 \backslash \mathrm{H}, 2.4043450024,1.3242476246,-1.9690$ $013407 \backslash \mathrm{H}, 0.5028020647,-1.9673793804,-2.1978502224 \backslash \mathrm{H}, 3.0540718304,2.402$ $3013137,-0.7169459221 \backslash \mathrm{H},-0.202466743,-3.277618871,-1.2475159412 \backslash \mathrm{H}, 3.76$ $17326835,0.7923641821,-0.9529136657 \backslash \mathrm{H}, 1.5679494424,-3.0686371829,-1.30$ $4171679 \backslash \mathrm{H}, 1.7375418552,0.4989493166,2.2424458805 \backslash \mathrm{H}, 0.7469330929,-1.694$ $7897307,2.1290741417 \backslash \mathrm{H}, 3.3751464516,0.3422478471,1.5576456011 \backslash \mathrm{H}, 1.8755$ $733229,-2.7449061117,1.236497996 \backslash \mathrm{H}, 2.6145739503,1.9459847501,1.7245710$ $527 \backslash \mathrm{H}, 0.1831256393,-3.2094454334,1.4187371187 \backslash \mathrm{H},-1.388542882,-3.389355$ $7619,0.5163005944 \backslash \mathrm{H},-3.0307277992,-3.1582483272,0.3936966087 \backslash \backslash$ Version $=$ IA $64 \mathrm{~L}-\mathrm{G} 03$ RevD . $01 \backslash$ State $=2-\mathrm{A} \backslash \mathrm{HF}=-805.8159768 \backslash \mathrm{S} 2=0.753778 \backslash \mathrm{S} 2-1=0 . \backslash \mathrm{S} 2 \mathrm{~A}=0.7$ $5001 \backslash \mathrm{RMSD}=8.544 \mathrm{e}-09 \backslash \mathrm{RMSF}=1.099 \mathrm{e}-05 \backslash \mathrm{Thermal}=0 . \backslash \mathrm{Dipole}=-1.1339426,-0.034$ $9142,0.7052614 \backslash P G=C 01 \quad[X(C 16 H 19 N 201)] \backslash \backslash @$

\section{4-amino-TMAO+}

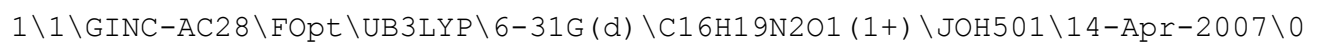

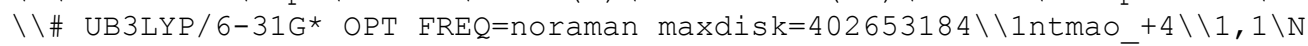
$, 1.7472743482,-0.5738398121,-0.4638755453 \backslash 0,2.5917932051,-0 . \overline{8} 890493629$ $,-1.2744651754 \backslash C, 0.5907794319,-1.5281838248,-0.2220393404 \backslash C, 0.31568639$ $66,-2.2437080473,-1.5656245672 \backslash C,-0.6813953919,0.6510138801,-0.0391366$ $746 \backslash C,-1.9274221804,1.3514839971,-0.1256862864 \backslash C,-1.9405206802,2.70264$ $49129,-0.5251167628 \backslash \mathrm{C},-3.1219798144,0.6425153879,0.1810567809 \backslash \mathrm{H},-2.894$ $05936,3.2181572078,-0.5997029129 \backslash \mathrm{H},-4.0731068481,1.1653400723,0.138987$ $834 \backslash C,-0.7634213783,3.3658526621,-0.8339173323 \backslash C,-3.077265966,-0.67288$ $36876,0.5432566139 \backslash \mathrm{H},-0.784793752,4.4040671346,-1.1485094369 \backslash \mathrm{H},-3.9922$ $251774,-1.1994108541,0.8011592982 \backslash \mathrm{C}, 0.4692094457,2.7117993192,-0.68550$ $04336 \backslash \mathrm{C},-1.8505934475,-1.4072735974,0.5535391104 \backslash \mathrm{H}, 1.3823979063,3.2680$ $425987,-0.8623326098 \backslash \mathrm{N},-1.9354924525,-2.7598065943,0.8043892535 \backslash \mathrm{C}, 0.52$ $13435624,1.3981682596,-0.2310272318 \backslash C,-0.6522569873,-0.7516026289,0.20$ $59342234 \backslash C, 1.8342677194,0.7651287843,0.2323996379 \backslash C, 1.8704092923,0.560$ $4928396,1.7605350806 \backslash \mathrm{C}, 1.182871649,-2.5515769454,0.8021290567 \backslash \mathrm{C}, 3.1000$ $209036,1.4717246205,-0.2470109322 \backslash \mathrm{H}, 3.0968770947,1.6464343607,-1.32531$ $33043 \backslash \mathrm{H}, 0.07071165,-1.5162762464,-2.3438074704 \backslash \mathrm{H}, 3.1822757739,2.431684$ $6275,0.2680209072 \backslash \mathrm{H},-0.5407409134,-2.9061483101,-1.4233031625 \backslash \mathrm{H}, 3.9845$ $240647,0.8802815436,0.0016320332 \backslash \mathrm{H}, 1.1767274117,-2.8342070667,-1.88391$ $09131 \backslash \mathrm{H}, 0.9759366167,0.0660486984,2.1417589678 \backslash \mathrm{H}, 1.1305748298,-2.19683$ $00277,1.8329448487 \backslash \mathrm{H}, 2.7609413414,-0.0008518185,2.0606680478 \backslash \mathrm{H}, 2.22423$ $69337,-2.7691991126,0.549796466 \backslash \mathrm{H}, 1.91875816,1.5526187722,2.2191213317$ $\backslash \mathrm{H}, 0.6461117453,-3.4976617768,0.7093945662 \backslash \mathrm{H},-1.1679028545,-3.22665443$ $14,1.2626441303 \backslash \mathrm{H},-2.8388822783,-3.1018255342,1.1069719031 \backslash \backslash$ Version=IA $64 \mathrm{~L}-\mathrm{G} 03 \mathrm{RevD} .01 \backslash \mathrm{State}=1-\mathrm{A} \backslash \mathrm{HF}=-805.5817419 \backslash \mathrm{S} 2=0 . \backslash \mathrm{S} 2-1=0 . \backslash \mathrm{S} 2 \mathrm{~A}=0 . \backslash \mathrm{RMSD}=6.2$ $09 \mathrm{e}-09 \backslash \mathrm{RMSF}=4.214 \mathrm{e}-06 \backslash \mathrm{Thermal}=0 . \backslash \mathrm{Dipole}=-0.1215176,-0.6277277,0.844722$ $4 \backslash \mathrm{PG}=\mathrm{C} 01 \quad[\mathrm{X}(\mathrm{C} 16 \mathrm{H} 19 \mathrm{~N} 2 \mathrm{O} 1)] \backslash \backslash \mathrm{Q}$ 


\section{4-amino-TMAO-}

$1 \backslash 1 \backslash G I N C-A C 56 \backslash F O p t \backslash U B 3 L Y P \backslash 6-31 G(d) \backslash C 16 H 19 N 2 O 1$ (1-) \JOH501\15-Apr-2007\0

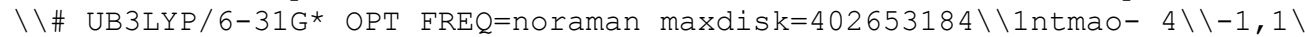
$\mathrm{N}, 1.7134693452,-0.6394208536,-0.5749016128 \backslash 0,2.9242544349,-1 . \overline{3} 23305379$ $2,-0.4148948888 \backslash \mathrm{C}, 0.584414536,-1.5325481313,-0.1491604318 \backslash \mathrm{C}, 0.53594779$ $31,-2.5398874758,-1.3324829658 \backslash \mathrm{C},-0.7498802277,0.6595804791,-0.1041630$ $516 \backslash \mathrm{C},-1.9985108806,1.3798966277,-0.1155914721 \backslash \mathrm{C},-2.0054750255,2.79742$ $29561,-0.187618265 \backslash \mathrm{C},-3.2076282762,0.6521252514,-0.0231245476 \backslash \mathrm{H},-2.964$ $4576283,3.3139067281,-0.206959183 \backslash \mathrm{H},-4.153710084,1.1911408989,-0.03789$ $59068 \backslash \mathrm{C},-0.8235365616,3.4959598778,-0.212785907 \backslash \mathrm{C},-3.185429092,-0.7118$ $185916,0.1143694789 \backslash \mathrm{H},-0.8240195246,4.5836091792,-0.2609839273 \backslash \mathrm{H},-4.11$ $63050328,-1.2708469106,0.2085988962 \backslash \mathrm{C}, 0.4047150895,2.7973547861,-0.156$ $6929214 \backslash \mathrm{C},-1.9617957877,-1.4333743643,0.1242201925 \backslash \mathrm{H}, 1.3276201366,3.36$ $87559923,-0.1491951142 \backslash \mathrm{N},-2.0735690924,-2.8375818847,0.2593141234 \backslash \mathrm{C}, 0$. $4687748264,1.4158386207,-0.1017186501 \backslash \mathrm{C},-0.7385242564,-0.7711505859,-0$ $.0339460215 \backslash C, 1.8044285412,0.7047171924,0.0447800911 \backslash C, 2.239128884,0.6$ $682830353,1.5377853704 \backslash C, 0.9532769934,-2.2922985001,1.1597860276 \backslash C, 2.9$ $202458381,1.4114685018,-0.7609733969 \backslash \mathrm{H}, 2.586513524,1.5961656977,-1.788$ $4550533 \backslash \mathrm{H}, 0.2188664845,-2.0173184258,-2.2410496154 \backslash \mathrm{H}, 3.2362281061,2.36$ $06763686,-0.3096830794 \backslash \mathrm{H},-0.1262203779,-3.3958657436,-1.1692917419 \backslash \mathrm{H}, 3$ $.7536991345,0.7062102845,-0.7864749552 \backslash \mathrm{H}, 1.5704018678,-2.8699830731,-1$ $.4626575456 \backslash \mathrm{H}, 1.4157725737,0.3680228343,2.1954956266 \backslash \mathrm{H}, 0.5857622369,-1$ $.7944754875,2.0640451333 \backslash \mathrm{H}, 3.0406480561,-0.0772021926,1.5906720268 \backslash \mathrm{H}, 2$ $.0566274608,-2.2894939846,1.1211277566 \backslash \mathrm{H}, 2.6086227801,1.642289449,1.88$ $53454397 \backslash \mathrm{H}, 0.6166104039,-3.3399343755,1.1792410476 \backslash \mathrm{H},-1.2342616126,-3$. $2580549023,0.6386267839 \backslash \mathrm{H},-2.8710355865,-3.0923538991,0.8333522599 \backslash \backslash \mathrm{Ve}$ rsion=IA64L-G03RevD.01 SState $=1-A \backslash H F=-805.8165882 \backslash S 2=0 . \backslash S 2-1=0 . \backslash S 2 A=0 . \backslash$ $\operatorname{RMSD}=6.994 \mathrm{e}-09 \backslash \mathrm{RMSF}=3.790 \mathrm{e}-06 \backslash \mathrm{Thermal}=0 . \backslash \mathrm{Dipole}=-2.8578342,0.8462037,1$ $.0144111 \backslash \mathrm{PG}=\mathrm{C} 01 \quad[\mathrm{X}(\mathrm{C} 16 \mathrm{H} 19 \mathrm{~N} 2 \mathrm{O} 1)] \backslash \backslash \mathrm{Q}$

\section{5-amino-TMAO• (50)}

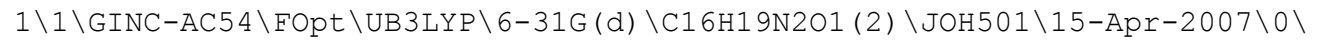

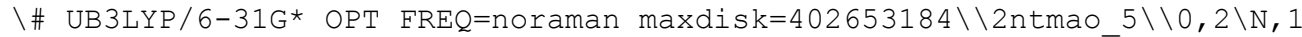
$.8503916834,-0.901912207,-0.2559258913 \backslash 0,2.9360916045,-1.584118696,-0$. $338985764 \backslash \mathrm{C}, 0.6180032512,-1.6952806135,0.0358930864 \backslash \mathrm{C}, 0.5711489925,-2$. $8110513987,-1.0310014724 \backslash C,-0.5433125997,0.60526465,-0.0224663655 \backslash C,-1$ $.7694685977,1.3596951402,-0.0204045771 \backslash C,-1.7086466781,2.7775535517,-0$ $.0045439209 \backslash \mathrm{C},-3.0151406935,0.6906502198,-0.0318297975 \backslash \mathrm{H},-2.6384898595$ $, 3.3410011498,-0.0033625893 \backslash \mathrm{H},-3.9285103868,1.2820368825,-0.0344288143$ $\backslash \mathrm{C},-0.496263952,3.4237864745,0.0173460846 \backslash \mathrm{C},-3.0794531668,-0.690001838$ $3,-0.0271974871 \backslash \mathrm{H},-0.4537704534,4.5096044595,0.0363677639 \backslash \mathrm{N},-4.2944171$ $549,-1.3766673739,0.0305197002 \backslash \mathrm{C}, 0.7047485294,2.683941154,0.0109175998$ $\backslash \mathrm{C},-1.8665114737,-1.4288666703,-0.0164578358 \backslash \mathrm{H}, 1.6469276686,3.22282078$ $35,0.0270524228 \backslash C, 0.7008972813,1.2998311657,-0.017257676 \backslash C,-0.62916855$ $88,-0.8199817633,-0.0210319121 \backslash \mathrm{C}, 2.0344466411,0.5617981723,-0.01995555$ $03 \backslash \mathrm{C}, 2.7593760409,0.7460768599,1.3356920022 \backslash \mathrm{C}, 0.7677320267,-2.31986428$ $26,1.4454473576 \backslash \mathrm{C}, 2.9369702393,1.0628120739,-1.1701455437 \backslash \mathrm{H}, 2.41383221$ $37,0.9852469923,-2.1283374154 \backslash \mathrm{H}, 0.4000033579,-2.3851393725,-2.02456749$ $91 \backslash \mathrm{H}, 3.2205023779,2.1068969431,-1.0143224602 \backslash \mathrm{H},-0.2292609143,-3.523331$ $8244,-0.8147543589 \backslash \mathrm{H}, 3.8409009589,0.4525358455,-1.2111297703 \backslash \mathrm{H}, 1.52322$ $99408,-3.3435503206,-1.0356610679 \backslash \mathrm{H}, 2.1240628523,0.4316856064,2.169665$ $2 \backslash \mathrm{H}, 0.7564014414,-1.5480033048,2.221260328 \backslash \mathrm{H}, 3.6746343228,0.148069027$, $1.341087237 \backslash \mathrm{H}, 1.7137333625,-2.8657884343,1.499970475 \backslash \mathrm{H}, 3.0180509172,1$. $7983485895,1.4878120512 \backslash \mathrm{H},-0.0574535718,-3.0103701869,1.6458904218 \backslash \mathrm{H},-$ $5.0986909994,-0.8447494527,-0.2786307966 \backslash \mathrm{H},-4.2832930915,-2.3018171809$ $,-0.3810261014 \backslash \mathrm{H},-1.9368935523,-2.5128108205,0.0086029367 \backslash \backslash$ Version=IA 6 4L-G03RevD.01 \State $=2-A \backslash H F=-805.8247186 \backslash S 2=0.753761 \backslash S 2-1=0 . \backslash S 2 A=0.7500$ $1 \backslash \mathrm{RMSD}=9.703 e-09 \backslash \mathrm{RMSF}=1.503 e-06 \backslash \mathrm{Thermal}=0 . \backslash \mathrm{Dipole}=-1.3497395,0.139489$, $-0.1738399 \backslash \mathrm{PG}=\mathrm{C} 01 \quad[\mathrm{X}(\mathrm{C} 16 \mathrm{H} 19 \mathrm{~N} 2 \mathrm{O} 1)] \backslash \backslash \mathrm{Q}$

\section{5-amino-TMAO+}

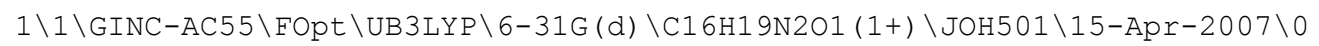




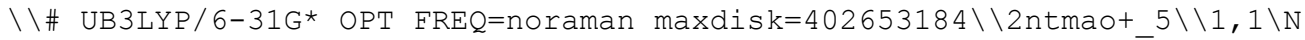
$, 1.7526859556,-0.9450369597,-0.3968337805 \backslash 0,2.5090117042,-1.5 \overline{2} 4829742$, $-1.146080999 \backslash \mathrm{C}, 0.5940623871,-1.7086731982,0.2236368965 \backslash \mathrm{C}, 0.6015835106$, $-3.1229962081,-0.3536249244 \backslash C,-0.5372526178,0.5503302847,0.0662487507 \backslash$ $C,-1.7534125821,1.3099418558,0.0787493098 \backslash C,-1.6930566663,2.6868629466$ $, 0.4371412955 \backslash \mathrm{C},-2.9630038057,0.6850344063,-0.2744014037 \backslash \mathrm{H},-2.61373092$ $62,3.2621384497,0.4704047107 \backslash \mathrm{H},-3.8765361934,1.2738322012,-0.286518166$ $1 \backslash \mathrm{C},-0.4885646478,3.2789108747,0.7382969338 \backslash \mathrm{C},-3.0079012973,-0.6693409$ $625,-0.6269152971 \backslash \mathrm{H},-0.4498838865,4.3250416966,1.0246627814 \backslash \mathrm{N},-4.17067$ $43454,-1.2622870298,-1.0246809247 \backslash C, 0.7156630501,2.5470978096,0.631918$ $8489 \backslash \mathrm{C},-1.8119329204,-1.4392028232,-0.5346049956 \backslash \mathrm{H}, 1.656063305,3.06358$ $26416,0.7986487485 \backslash \mathrm{C}, 0.7014140368,1.209874153,0.2664979583 \backslash \mathrm{C},-0.621817$ $3125,-0.8579319946,-0.1545786827 \backslash C, 1.9964816155,0.5281015088,-0.140476$ $8143 \backslash \mathrm{C}, 3.1176977118,0.585390813,0.9351527867 \backslash \mathrm{C}, 0.7901808749,-1.7496381$ $157,1.7567750996 \backslash \mathrm{C}, 2.5171508462,1.1287172977,-1.4687560641 \backslash \mathrm{H}, 1.7638972$ $735,1.035334854,-2.255949318 \backslash \mathrm{H}, 0.4486528292,-3.1295401575,-1.435686567$ $5 \backslash \mathrm{H}, 2.7181134785,2.1897161474,-1.3108720009 \backslash \mathrm{H},-0.1954714513,-3.6992938$ $543,0.1215915031 \backslash \mathrm{H}, 3.437553175,0.6375665104,-1.7883818056 \backslash \mathrm{H}, 1.54743545$ $,-3.6237079095,-0.1360386578 \backslash \mathrm{H}, 2.7883264612,0.2116877605,1.9058184523 \backslash$ $\mathrm{H}, 0.7659476971,-0.7565420562,2.2075781595 \backslash \mathrm{H}, 3.9847286421,0.0123736153$, $0.5951371931 \backslash \mathrm{H}, 1.7260784868,-2.2533980015,2.0189020789 \backslash \mathrm{H}, 3.4241484929$, $1.6270725694,1.0541402501 \backslash \mathrm{H},-0.0399663352,-2.3254347274,2.1765313524 \backslash \mathrm{H}$ $,-5.0518622708,-0.7840521575,-0.9069676224 \backslash \mathrm{H},-4.2218020069,-2.26223487$ $02,-1.1496602011 \backslash \mathrm{H},-1.8766677187,-2.5081176286,-0.7067048843 \backslash \backslash$ Version $=$ IA 64L-G03RevD. $01 \backslash$ State $=1-A \backslash H F=-805.5866712 \backslash \mathrm{S} 2=0 . \backslash \mathrm{S} 2-1=0 . \backslash \mathrm{S} 2 \mathrm{~A}=0 . \backslash \mathrm{RMSD}=8$ $.118 \mathrm{e}-09 \backslash \mathrm{RMSF}=1.250 \mathrm{e}-05 \backslash \mathrm{Thermal}=0 . \backslash \mathrm{Dipole}=-0.5316217,-0.5257466,0.3049$ $128 \backslash \mathrm{PG}=\mathrm{C} 01 \quad[\mathrm{X}(\mathrm{C} 16 \mathrm{H} 19 \mathrm{~N} 2 \mathrm{O} 1)] \backslash \backslash @$

\section{5-amino-TMAO-}

$1 \backslash 1 \backslash G I N C-A C 55 \backslash F O p t \backslash U B 3 L Y P \backslash 6-31 G(d) \backslash C 16 H 19 N 2 O 1$ (1-) \JOH501\15-Apr-2007 \0

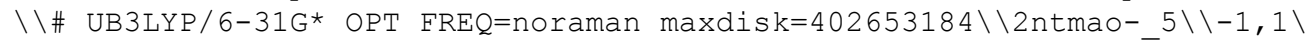
$\mathrm{N}, 1.7824255752,-0.8619149993,-0.5603204505 \backslash 0,2.9649593058,-1 . \overline{5} 96141070$ $5,-0.4019240437 \backslash \mathrm{C}, 0.6379639804,-1.6384467854,-0.0128123253 \backslash \mathrm{C}, 0.5326779$ $37,-2.8463581013,-0.9778829971 \backslash C,-0.5678077397,0.6257995538,-0.0497058$ $9 \backslash \mathrm{C},-1.7946477792,1.3801827543,-0.0303895856 \backslash \mathrm{C},-1.7328992451,2.7966799$ $157,-0.0297012109 \backslash \mathrm{C},-3.0397083309,0.6993485846,-0.011694353 \backslash \mathrm{H},-2.66288$ $17178,3.3635205992,-0.0150815539 \backslash \mathrm{H},-3.961061675,1.2819089883,-0.004501$ $2954 \backslash \mathrm{C},-0.5144399438,3.4402747927,-0.0398255733 \backslash \mathrm{C},-3.085233256,-0.6789$ $835707,0.0079463027 \backslash \mathrm{H},-0.4720300393,4.5284826656,-0.0326655493 \backslash \mathrm{N},-4.31$ $17220823,-1.3813397921,0.087690625 \backslash \mathrm{C}, 0.6857671119,2.6984199493,-0.0588$ $969305 \backslash \mathrm{C},-1.8720434792,-1.4157924014,0.0007884803 \backslash \mathrm{H}, 1.6327904736,3.229$ $5166151,-0.0692358722 \backslash \mathrm{C}, 0.6839468707,1.3117368985,-0.0637722366 \backslash \mathrm{C},-0.6$ $345690608,-0.8034544053,-0.0347631961 \backslash C, 1.9851647544,0.5265549594,-0.0$ $589321524 \backslash \mathrm{C}, 2.6313959616,0.5607730373,1.353372556 \backslash \mathrm{C}, 0.9022063686,-2.19$ $08802751,1.4176310811 \backslash \mathrm{C}, 2.9969490527,1.1236006303,-1.0684366095 \backslash \mathrm{H}, 2.52$ $68916632,1.2347698843,-2.0520943408 \backslash \mathrm{H}, 0.2144486391,-2.5095912565,-1.97$ $1095172 \backslash \mathrm{H}, 3.3987127835,2.0941570897,-0.7506222518 \backslash \mathrm{H},-0.1566909923,-3.6$ $231316823,-0.6222718727 \backslash \mathrm{H}, 3.7992331717,0.3852360858,-1.144178154 \backslash \mathrm{H}, 1.5$ $477470062,-3.2444567958,-1.0515149318 \backslash \mathrm{H}, 1.8945820722,0.415384503,2.150$ $282084 \backslash \mathrm{H}, 0.7269029376,-1.4365582819,2.1919482191 \backslash \mathrm{H}, 3.342251294,-0.2759$ $004292,1.3439922859 \backslash \mathrm{H}, 1.9611276861,-2.4765782527,1.4104323494 \backslash \mathrm{H}, 3.1530$ $631244,1.5072673748,1.5468967036 \backslash \mathrm{H}, 0.2732773792,-3.0608343619,1.648281$ $3547 \backslash \mathrm{H},-5.0880892553,-0.8497250169,-0.2927940817 \backslash \mathrm{H},-4.266324802,-2.286$ $1590228,-0.3702183683 \backslash \mathrm{H},-1.9309957503,-2.5010183806,0.0261689564 \backslash \backslash$ Vers ion=IA $64 \mathrm{~L}-\mathrm{G} 03 \mathrm{RevD} .01 \backslash$ State $=1-\mathrm{A} \backslash \mathrm{HF}=-805.8231425 \backslash \mathrm{S} 2=0 . \backslash \mathrm{S} 2-1=0 . \backslash \mathrm{S} 2 \mathrm{~A}=0 . \backslash \mathrm{RM}$ $\mathrm{SD}=7.611 \mathrm{e}-09 \backslash \mathrm{RMSF}=6.740 \mathrm{e}-06 \backslash \mathrm{Thermal}=0 . \backslash \mathrm{Dipole}=-3.2536357,1.1512352,0.0$ $201902 \backslash P G=C 01 \quad[X(C 16 H 19 N 201)] \backslash \backslash @$

\section{6-amino-TMAO• $(51)$}

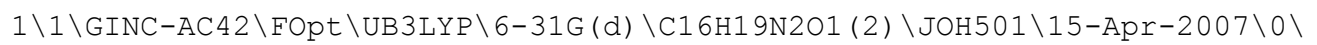

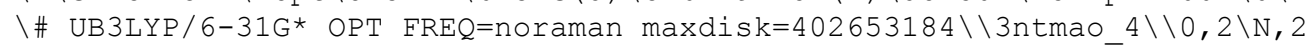
$.11642,-0.27308,-0.27824 \backslash 0,3.38164,-0.46941,-0.38625 \backslash \mathrm{C}, 1.30 \overline{1} 47,-1.4793$ $9,0.05494 \backslash C, 1.69617,-1.95431,1.4759 \backslash C,-0.68234,0.17408,-0.014 \backslash C,-2.104$ 
$26,0.40898,-0.00555 \backslash C,-2.59121,1.7372,0.07001 \backslash C,-3.00734,-0.7043,-0.05$ $433 \backslash \mathrm{H},-3.65913,1.91333,0.14724 \backslash \mathrm{N},-4.39161,-0.48126,-0.0579 \backslash \mathrm{C},-1.72227$, $2.8028,0.10305 \backslash \mathrm{C},-2.48949,-1.98554,-0.03372 \backslash \mathrm{H},-2.10077,3.81867,0.17451$ $\backslash \mathrm{H},-3.16231,-2.84005,-0.05881 \backslash \mathrm{C},-0.33377,2.57844,0.05047 \backslash \mathrm{C},-1.10059,-2$ $.20241,0.00574 \backslash \mathrm{H}, 0.33089,3.43663,0.06824 \backslash \mathrm{H},-0.75044,-3.2296,0.02986 \backslash \mathrm{C}$, $0.19491,1.30062,-0.00856 \backslash \mathrm{C},-0.18804,-1.16518,-0.00741 \backslash \mathrm{C}, 1.71183,1.1457$ $2,-0.04929 \backslash \mathrm{C}, 2.34454,1.61992,1.28218 \backslash \mathrm{C}, 2.30886,1.94806,-1.22843 \backslash \mathrm{C}, 1.69$ $445,-2.55536,-0.98063 \backslash \mathrm{H}, 1.8327,1.65644,-2.16979 \backslash \mathrm{H}, 1.37546,-2.25688,-1$. $98405 \backslash \mathrm{H}, 2.16113,3.02146,-1.08342 \backslash \mathrm{H}, 1.22959,-3.51481,-0.73943 \backslash \mathrm{H}, 3.37881$ $, 1.74374,-1.29677 \backslash \mathrm{H}, 2.77815,-2.68118,-0.97868 \backslash \mathrm{H}, 1.90825,1.09346,2.1367$ $2 \backslash \mathrm{H}, 1.38921,-1.2279,2.23494 \backslash \mathrm{H}, 3.42028,1.4271,1.25817 \backslash \mathrm{H}, 2.78076,-2.0871$ $8,1.52802 \backslash \mathrm{H}, 2.17704,2.6919,1.42481 \backslash \mathrm{H}, 1.20742,-2.9066,1.70543 \backslash \mathrm{H},-4.6934$ $1,0.27853,-0.65691 \backslash \mathrm{H},-4.92797,-1.31741,-0.25946 \backslash \backslash$ Version=IA64L-G03RevD $.01 \backslash \mathrm{State}=2-\mathrm{A} \backslash \mathrm{HF}=-805.8222015 \backslash \mathrm{S} 2=0.753753 \backslash \mathrm{S} 2-1=0 . \backslash \mathrm{S} 2 \mathrm{~A}=0.75001 \backslash \mathrm{RMSD}=6.3$ $49 e-09 \backslash \mathrm{RMSF}=3.865 e-06 \backslash \mathrm{Thermal}=0 . \backslash \mathrm{Dipol} e=-1.4835794,0.096067,-0.176608 \backslash$ $\mathrm{PG}=\mathrm{C} 01 \quad[\mathrm{X}(\mathrm{C} 16 \mathrm{H} 19 \mathrm{~N} 2 \mathrm{O} 1)] \backslash \backslash @$

\section{6-amino-TMAO+}

$1 \backslash 1 \backslash G I N C-A C 53 \backslash F O p t \backslash U B 3 L Y P \backslash 6-31 G(d) \backslash C 16 H 19 N 2 O 1(1+) \backslash J O H 501 \backslash 15-A p r-2007 \backslash 0$

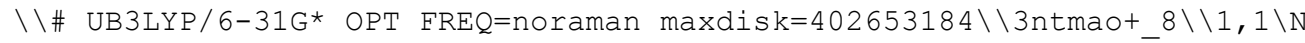
$,-0.0076239179,0.0386844036,-0.014325518 \backslash 0,1.2039625104,0.134 \overline{4} 061969,-$ $0.0246390554 \backslash \mathrm{C},-2.445346736,1.3552897646,-0.0095970951 \backslash \mathrm{C},-3.5717899906$ $, 2.2351251474,0.0003969979 \backslash \mathrm{C},-4.4357307908,2.2140095365,1.1192048521 \backslash \mathrm{C}$ $,-3.7626949642,3.1150290189,-1.1311254486 \backslash N,-4.8022839319,3.9810229345$ $,-1.182960061 \backslash \mathrm{C},-4.1783384494,1.3935058796,2.2003538723 \backslash \mathrm{C},-2.843716791$ $5,3.0664614011,-2.1950930779 \backslash \mathrm{H},-4.8589717612,1.3763297907,3.0450982446$ $\backslash \mathrm{H},-2.9766684865,3.7378035954,-3.0384432055 \backslash \mathrm{C},-3.003235983,0.621317762$ $7,2.2378496602 \backslash \mathrm{C},-1.810132739,2.1357885729,-2.2199077283 \backslash \mathrm{H},-1.17966931$ $66,2.0925184228,-3.1008859558 \backslash C,-2.1236425954,0.6265593814,1.167220567$ $2 \backslash C,-1.6369440815,1.2237301203,-1.1786062108 \backslash C,-0.7168562528,0.0685848$ $803,1.3278060953 \backslash C,-0.7746771686,-0.0259025661,-1.3216445602 \backslash C,-0.6584$ $744078,-1.3884580696,1.8598313872 \backslash \mathrm{C},-1.6069896702,-1.3198168014,-1.387$ $893629 \backslash \mathrm{C}, 0.1067439322,0.9968544925,2.2498009046 \backslash \mathrm{C}, 0.235508745,0.023036$ $1681,-2.4659487975 \backslash \mathrm{H}, 0.1350385098,2.0139100327,1.8489984949 \backslash \mathrm{H}, 0.831930$ $8892,0.9383962251,-2.4563690634 \backslash \mathrm{H},-0.3743868027,1.0261037963,3.2295396$ $9 \backslash \mathrm{H},-0.3045272451,-0.0400870962,-3.4138382564 \backslash \mathrm{H}, 1.1273675197,0.6296076$ $211,2.3675919033 \backslash \mathrm{H}, 0.917180192,-0.8288656735,-2.4097382482 \backslash \mathrm{H},-1.255389$ $5305,-2.0788351002,1.2619525826 \backslash \mathrm{H},-2.3522540423,-1.3829566904,-0.59354$ $88781 \backslash \mathrm{H}, 0.3795991717,-1.7309371956,1.8846712803 \backslash \mathrm{H},-0.9622549827,-2.204$ $3967606,-1.3588194039 \backslash \mathrm{H},-1.0422063038,-1.3985420865,2.8829591812 \backslash \mathrm{H},-2$. $1425767531,-1.3172333118,-2.3423293322 \backslash \mathrm{H},-2.7745058435,0.0585524963,3$. $1378745307 \backslash \mathrm{H},-5.3252738309,2.8355911876,1.1448736876 \backslash \mathrm{H},-4.8886937914,4$ $.6254600963,-1.9555675641 \backslash \mathrm{H},-5.4166243089,4.1372424266,-0.3998828428 \backslash \backslash$ Version=IA64L-G03RevD.01 \State $=1-A \backslash H F=-805.5901813 \backslash \mathrm{S} 2=0 . \backslash \mathrm{S} 2-1=0 . \backslash \mathrm{S} 2 \mathrm{~A}=0$ $. \backslash \mathrm{RMSD}=8.602 \mathrm{e}-09 \backslash \mathrm{RMSF}=4.302 \mathrm{e}-06 \backslash \mathrm{Thermal}=0 . \backslash \mathrm{Dipole}=-0.9018022,0.4679895$ ,$-0.1656639 \backslash \mathrm{PG}=\mathrm{CO} 1 \quad[\mathrm{X}(\mathrm{C} 16 \mathrm{H} 19 \mathrm{~N} 2 \mathrm{O} 1)] \backslash \backslash @$

\section{6-amino-TMAO-}

$1 \backslash 1 \backslash G I N C-A C 22 \backslash$ FOpt \UB3LYP \6-31G (d) \C16H19N2O1 (1-) \JOH501 \15-Apr-2007\0

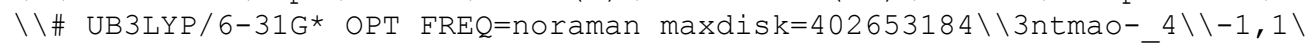
$\mathrm{N}, 2.0392813108,-0.2654002648,-0.5631076098 \backslash 0,3.4161192221,-0 . \overline{4} 70801659$ $3,-0.4212748127 \backslash C, 1.2952533212,-1.4212891037,0.0038534776 \backslash C, 1.76878493$ $29,-1.799978996,1.4355661436 \backslash \mathrm{C},-0.7087686303,0.1819245931,-0.041373769$ $\backslash C,-2.1328668832,0.4158526618,-0.0103218257 \backslash C,-2.6195749857,1.74734850$ $84,0.0358171734 \backslash \mathrm{C},-3.0263426051,-0.7015867055,-0.0169417383 \backslash \mathrm{H},-3.68774$ $48702,1.9251912009,0.1265958835 \backslash N,-4.4312250251,-0.477025064,-0.002859$ $1733 \backslash \mathrm{C},-1.7431997818,2.8097125935,0.0265992794 \backslash \mathrm{C},-2.5065475036,-1.9784$ $946417,0.012678852 \backslash \mathrm{H},-2.1207125682,3.8297100982,0.0768751507 \backslash \mathrm{H},-3.1826$ $426841,-2.8340861461,0.0120914938 \backslash \mathrm{C},-0.3522766736,2.5824076718,-0.0357$ $356893 \backslash \mathrm{C},-1.1107049828,-2.1951429867,0.028886682 \backslash \mathrm{H}, 0.3192879686,3.4355$ $724326,-0.0489797891 \backslash \mathrm{H},-0.7496309341,-3.2189508419,0.0539061697 \backslash \mathrm{C}, 0.17$ $88599253,1.3032088257,-0.0631742766 \backslash C,-0.202107176,-1.1553621226,-0.01$ $537754 \backslash \mathrm{C}, 1.6852304379,1.0961386223,-0.0802150595 \backslash \mathrm{C}, 2.2928747479,1.4140$ 
$759468,1.3155084552 \backslash \mathrm{C}, 2.3584800064,2.0270987813,-1.1208704332 \backslash \mathrm{C}, 1.6675$ $107497,-2.5865229027,-0.946634661 \backslash \mathrm{H}, 1.8659228476,1.9232806784,-2.09431$ $96422 \backslash \mathrm{H}, 1.2379712671,-2.4143910929,-1.9400543157 \backslash \mathrm{H}, 2.3491279104,3.0835$ $456227,-0.8237152622 \backslash \mathrm{H}, 1.336678712,-3.5652600756,-0.5760043183 \backslash \mathrm{H}, 3.386$ $2134558,1.6653936623,-1.2076432696 \backslash \mathrm{H}, 2.7570988453,-2.5596734261,-1.026$ $8049919 \backslash \mathrm{H}, 1.6863613465,1.0117612469,2.1337824672 \backslash \mathrm{H}, 1.3141891015,-1.164$ $0626979,2.2025146333 \backslash \mathrm{H}, 3.2733770178,0.9209420642,1.2988988059 \backslash \mathrm{H}, 2.8547$ $649331,-1.6445405102,1.419252847 \backslash \mathrm{H}, 2.4084839139,2.4931910958,1.4816221$ $112 \backslash \mathrm{H}, 1.5376054902,-2.8438365416,1.6859276649 \backslash \mathrm{H},-4.7062211091,0.229175$ $2136,-0.6806196533 \backslash \mathrm{H},-4.9338810507,-1.3338957409,-0.21574946 \backslash \backslash$ Version= IA 64 L-G03RevD.01 \State $=1-\mathrm{A} \backslash \mathrm{HF}=-805.8199112 \backslash \mathrm{S} 2=0 . \backslash \mathrm{S} 2-1=0 . \backslash \mathrm{S} 2 \mathrm{~A}=0 . \backslash \mathrm{RMSD}=2$ $.757 e-09 \backslash \mathrm{RMSF}=6.557 e-06 \backslash \mathrm{Thermal}=0 . \backslash \mathrm{Dipole}=-3.6186662,0.3126148,0.02765$ $\backslash \mathrm{PG}=\mathrm{C} 01 \quad[\mathrm{X}(\mathrm{C} 16 \mathrm{H} 19 \mathrm{~N} 2 \mathrm{O} 1)] \backslash \backslash @$

\section{4-nitro-TMAO• (52)}

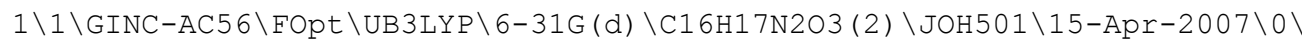

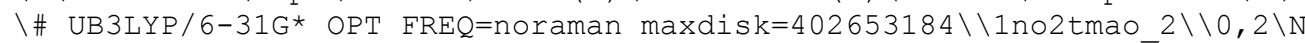
$, 0.0070653662,0.0025866447,0.049514106 \backslash 0,1.2898865984,-0.0178859629,0$. $0819631969 \backslash \mathrm{C},-0.6181996889,1.3671639076,-0.0746475765 \backslash \mathrm{C}, 0.0896102517,2$ $.2316860042,0.9942855472 \backslash C,-2.8237608828,0.0249873003,-0.0360311161 \backslash C$, $-4.2590112552,-0.0289450326,0.0820302975 \backslash C,-4.953454217,-1.2359982936$, $-0.1742058981 \backslash \mathrm{C},-4.977600771,1.130440863,0.4638566622 \backslash \mathrm{H},-6.0363197906$, $-1.2431701549,-0.0833849508 \backslash \mathrm{H},-6.0565431698,1.0723940924,0.5752598945 \backslash$ $C,-4.266266646,-2.3694534465,-0.5326774669 \backslash \mathrm{C},-4.3176028634,2.303830967$ $2,0.6948920968 \backslash \mathrm{H},-4.794401742,-3.2954519028,-0.7410921831 \backslash \mathrm{H},-4.8413365$ $283,3.2005206048,1.0060662553 \backslash C,-2.8605691349,-2.3369231092,-0.6062012$ $07 \backslash \mathrm{C},-2.9201963331,2.3654887433,0.493654704 \backslash \mathrm{H},-2.3359594244,-3.2527178$ $493,-0.85892307 \backslash N,-2.3981478276,3.7367010115,0.6889278073 \backslash \mathrm{C},-2.1339064$ $675,-1.1866949426,-0.3482735336 \backslash \mathrm{C},-2.1342936134,1.2748103313,0.1468009$ $271 \backslash \mathrm{C},-0.6167545678,-1.2815316446,-0.3734489561 \backslash \mathrm{C},-0.0999234379,-1.648$ $8563072,-1.7860673164 \backslash \mathrm{C},-0.3074023012,1.8918284708,-1.4997397605 \backslash \mathrm{C},-0$. $1322469452,-2.3374414441,0.6492712869 \backslash \mathrm{H},-0.5187190399,-2.1094165908,1$. $6474200936 \backslash \mathrm{H},-0.2835688762,2.0000898201,1.9966156859 \backslash \mathrm{H},-0.4715866773,-$ $3.3370880139,0.3654526645 \backslash \mathrm{H},-0.035886186,3.295210305,0.7979765574 \backslash \mathrm{H}, 0$. $9586463919,-2.3285545898,0.6836386892 \backslash \mathrm{H}, 1.1545713324,2.0047685262,0.95$ $90253036 \backslash \mathrm{H},-0.4754370746,-0.9549126222,-2.5433427007 \backslash \mathrm{H},-0.8139250372,1$ $.2898035843,-2.2605570126 \backslash \mathrm{H}, 0.9922488205,-1.6167250831,-1.7854334365 \backslash \mathrm{H}$ $, 0.7724540383,1.8325355891,-1.6638797446 \backslash \mathrm{H},-0.4238910554,-2.6570957359$ $,-2.0612426984 \backslash \mathrm{H},-0.6393159609,2.9251123649,-1.6036155629 \backslash 0,-2.5639710$ $968,4.2322339423,1.7999328317 \backslash 0,-1.9014841868,4.3192796529,-0.27383041$

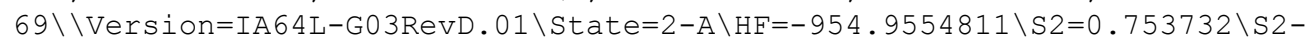
$1=0 . \backslash \mathrm{S} 2 \mathrm{~A}=0.75001 \backslash \mathrm{RMSD}=6.304 \mathrm{e}-09 \backslash \mathrm{RMSF}=2.677 \mathrm{e}-06 \backslash \mathrm{Thermal}=0 . \backslash \mathrm{Dipole}=-1.37$ $33268,-1.5789634,-0.5119011 \backslash \mathrm{PG}=\mathrm{C} 01 \quad[\mathrm{X}(\mathrm{C} 16 \mathrm{H} 17 \mathrm{~N} 2 \mathrm{O} 3)] \backslash \backslash \mathrm{Q}$

\section{4-nitro-TMAO+}

$1 \backslash 1 \backslash G I N C-A C 31 \backslash F O p t \backslash U B 3 L Y P \backslash 6-31 G(d) \backslash C 16 H 17 N 2 O 3(1+) \backslash J O H 501 \backslash 21-A p r-2007 \backslash 0$

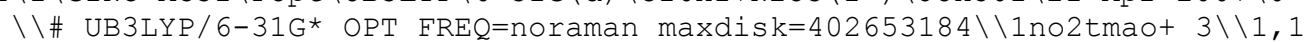
$\backslash \mathrm{N},-0.0105706506,-0.1301431952,0.1118464338 \backslash 0,1.0193493537,-0.3 \overline{6} 446609$ $73,0.6849466746 \backslash \mathrm{C},-0.626188495,1.2594518731,0.2930104272 \backslash \mathrm{C},-0.36664477$ $46,1.6346413188,1.7737834096 \backslash \mathrm{C},-2.7833162717,-0.0785133138,0.073668782$ $6 \backslash C,-4.1987958612,-0.1693703535,0.2694043579 \backslash C,-4.7903843771,-1.440222$ $6354,0.4877242676 \backslash \mathrm{C},-4.9779646442,1.0122975487,0.2162476085 \backslash \mathrm{H},-5.86157$ $60987,-1.4950168571,0.6585079864 \backslash \mathrm{H},-6.0550168124,0.9489036814,0.336816$ $744 \backslash \mathrm{C},-4.0240452461,-2.5853028744,0.4816432473 \backslash \mathrm{C},-4.3776642497,2.22196$ $34941,-0.0229543964 \backslash \mathrm{H},-4.4803115072,-3.5537049398,0.6590667732 \backslash \mathrm{H},-4.95$ $88656467,3.1303188911,-0.1294359248 \backslash \mathrm{C},-2.6485018098,-2.5134048995,0.17$ $80629949 \backslash \mathrm{C},-2.9692623747,2.3103034781,-0.0727601162 \backslash \mathrm{H},-2.0858821427,-3$ $.4351742265,0.0877177643 \backslash \mathrm{N},-2.4841588248,3.7005873238,-0.2377242701 \backslash \mathrm{C}$, $-2.0463265682,-1.292658904,-0.0784434131 \backslash C,-2.1355911049,1.1978195685$, $0.0267849687 \backslash \mathrm{C},-0.658730659,-1.2147929619,-0.7199667762 \backslash \mathrm{C},-0.735224959$ $5,-0.7432213143,-2.1912322594 \backslash C, 0.2233860284,2.1432882747,-0.669866169$ $\backslash \mathrm{C}, 0.1883612668,-2.4793644979,-0.6036359822 \backslash \mathrm{H}, 0.253021198,-2.843824177$ $5,0.4246609434 \backslash \mathrm{H},-0.8315796118,0.9026341777,2.4405871606 \backslash \mathrm{H},-0.25654577$ 
$83,-3.2570922085,-1.2284011476 \backslash \mathrm{H},-0.8082628602,2.6132614312,1.95140031$ $94 \backslash \mathrm{H}, 1.2015662762,-2.2981242774,-0.9696566457 \backslash \mathrm{H}, 0.7041711908,1.6818037$ $255,1.9781386177 \backslash \mathrm{H},-1.3322138833,0.160695038,-2.3185728796 \backslash \mathrm{H},-0.015086$ $1649,1.9636761366,-1.7199813722 \backslash \mathrm{H}, 0.266437943,-0.5854221543,-2.6024338$ $047 \backslash \mathrm{H}, 1.2846359995,1.947003021,-0.4975106724 \backslash \mathrm{H},-1.2191062568,-1.543732$ $0345,-2.7585337735 \backslash \mathrm{H}, 0.0353167959,3.1898067476,-0.4327157691 \backslash 0,-2.9718$ $009221,4.3247519292,-1.1687825926 \backslash 0,-1.6675074957,4.1437842636,0.56988$ $84831 \backslash \backslash$ Version=IA64L-G03RevD. 01 \State=1-A \HF=-954.7028333 $\backslash \mathrm{S} 2=0 . \backslash \mathrm{S} 2-1=0$ $. \backslash \mathrm{S} 2 \mathrm{~A}=0 . \backslash \mathrm{RMSD}=3.686 \mathrm{e}-09 \backslash \mathrm{RMSF}=1.716 \mathrm{e}-05 \backslash \mathrm{Thermal}=0 . \backslash \mathrm{Dipole}=0.1936551,-2$. $4038916,-0.1832303 \backslash \mathrm{PG}=\mathrm{C} 01 \quad[\mathrm{X}(\mathrm{C} 16 \mathrm{H} 17 \mathrm{~N} 2 \mathrm{O} 3)] \backslash \backslash @$

\section{4-nitro-TMAO-}

$1 \backslash 1 \backslash G I N C-A C 54 \backslash F O p t \backslash U B 3 L Y P \backslash 6-31 G(d) \backslash C 16 H 17 N 2 O 3(1-) \backslash J O H 501 \backslash 17-A p r-2007 \backslash 0$

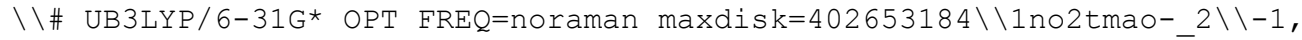
$1 \backslash \mathrm{N},-0.0575602238,-0.0290931543,0.2497397243 \backslash 0,1.2978135342,-0 . \overline{0} 580828$ $528,0.150738245 \backslash \mathrm{C},-0.6065981931,1.3260678743,-0.0537092279 \backslash \mathrm{C}, 0.0641943$ $89,2.2172395283,1.0269128182 \backslash C,-2.8363073968,0.0183461372,-0.007262207$ $3 \backslash \mathrm{C},-4.2721716176,-0.0495849909,0.1408128979 \backslash \mathrm{C},-4.9630501491,-1.259315$ $118,-0.0868804729 \backslash \mathrm{C},-4.9844403234,1.1188074614,0.5239319596 \backslash \mathrm{H},-6.04588$ $23348,-1.2662446488,0.0254287174 \backslash \mathrm{H},-6.0613967258,1.0593340736,0.667430$ $7881 \backslash \mathrm{C},-4.2809606617,-2.4019452902,-0.4481241323 \backslash \mathrm{C},-4.3254555516,2.298$ $047267,0.6920192801 \backslash \mathrm{H},-4.813105471,-3.3311580879,-0.6395534929 \backslash \mathrm{H},-4.84$ $38178967,3.2036889318,0.9824386362 \backslash \mathrm{C},-2.87732817,-2.3614508095,-0.5433$ $082909 \backslash \mathrm{C},-2.928157021,2.3898757742,0.4353001493 \backslash \mathrm{H},-2.3425829862,-3.273$ $2456211,-0.792435647 \backslash \mathrm{N},-2.4522854106,3.7611421534,0.5186085549 \backslash \mathrm{C},-2.15$ $33271689,-1.2020266217,-0.3157234855 \backslash C,-2.1379190534,1.2697323481,0.12$ $53429998 \backslash \mathrm{C},-0.6435564406,-1.2413238496,-0.3569441949 \backslash \mathrm{C},-0.1355291497,-$ $1.4587529425,-1.8081185156 \backslash \mathrm{C},-0.1849557668,1.8114578645,-1.4711911076 \backslash$ $\mathrm{C},-0.1210288449,-2.4014996155,0.5307519825 \backslash \mathrm{H},-0.5576652871,-2.33158438$ $33,1.5326646279 \backslash \mathrm{H},-0.3695623622,2.0087930693,2.0120074111 \backslash \mathrm{H},-0.3524917$ $725,-3.3861795871,0.1092519417 \backslash \mathrm{H},-0.0162324818,3.2809911057,0.80693396$ $15 \backslash \mathrm{H}, 0.9599647891,-2.2703777434,0.6100666543 \backslash \mathrm{H}, 1.1131369516,1.91795292$ $04,1.0450579939 \backslash \mathrm{H},-0.6451149261,-0.8065428417,-2.5230445895 \backslash \mathrm{H},-0.79770$ $01742,1.3417124869,-2.2489074208 \backslash \mathrm{H}, 0.9327136245,-1.2162311904,-1.79064$ $4022 \backslash \mathrm{H}, 0.8573285241,1.5088868639,-1.6062014965 \backslash \mathrm{H},-0.2818485047,-2.4961$ $69106,-2.1336555452 \backslash \mathrm{H},-0.2818214253,2.8913248322,-1.5745394918 \backslash 0,-2.90$ $8238124,4.4673065633,1.4444037874 \backslash 0,-1.694260197,4.2127111991,-0.35960$

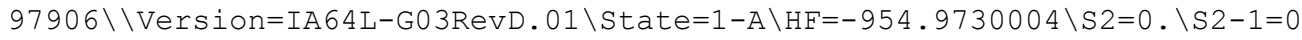
$. \backslash \mathrm{S} 2 \mathrm{~A}=0 . \backslash \mathrm{RMSD}=5.829 \mathrm{e}-09 \backslash \mathrm{RMSF}=2.411 \mathrm{e}-05 \backslash \mathrm{Thermal}=0 . \backslash \mathrm{Dipole}=-2.3026062,-1$ $.6240742,-0.5839804 \backslash \mathrm{PG}=\mathrm{C} 01 \quad[\mathrm{X}(\mathrm{C} 16 \mathrm{H} 17 \mathrm{~N} 2 \mathrm{O} 3)] \backslash \backslash @$

\section{5-nitro-TMAO• (53)}

$1 \backslash 1 \backslash G I N C-A C 39 \backslash F O p t \backslash U B 3 L Y P \backslash 6-31 G(d) \backslash C 16 H 17 N 203$ (2) \JOH501\15-Apr-2007\0 \

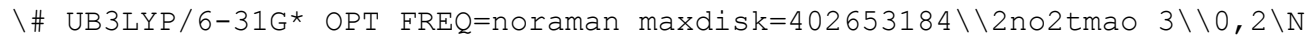
$, 1.8587077548,-0.8976717897,-0.2600870045 \backslash 0,2.9462000433,-1.5753580556$ $,-0.3414824679 \backslash \mathrm{C}, 0.6290337532,-1.6976049021,0.0208034759 \backslash \mathrm{C}, 0.575369535$ $8,-2.7933045813,-1.066712842 \backslash C,-0.5415941877,0.6038832084,-0.031695933$ $5 \backslash C,-1.76229233,1.3696685271,-0.0348238262 \backslash C,-1.7090056754,2.786790363$ $7,-0.0306479595 \backslash \mathrm{C},-3.0101683555,0.704954965,-0.0305595669 \backslash \mathrm{H},-2.6405548$ $244,3.3459959669,-0.0347257091 \backslash \mathrm{H},-3.937376233,1.2655724466,-0.03498854$ $56 \backslash C,-0.4962018362,3.4310312115,-0.0140614692 \backslash C,-3.0332288639,-0.66673$ $68687,-0.0136556565 \backslash \mathrm{H},-0.4478756334,4.5160142691,-0.0042627966 \backslash \mathrm{N},-4.33$ $02950898,-1.3602305481,-0.0004665915 \backslash C, 0.6998019668,2.6829682104,-0.01$ $23093856 \backslash \mathrm{C},-1.8550316602,-1.4374110929,-0.0062563696 \backslash \mathrm{H}, 1.6424098836,3$. $2206615883,0.0026891042 \backslash \mathrm{C}, 0.7030490538,1.2982853838,-0.0296427873 \backslash \mathrm{C},-0$ $.6188884705,-0.8239171381,-0.0212834213 \backslash \mathrm{C}, 2.038179316,0.5642605975,-0$. $0126169414 \backslash \mathrm{C}, 2.7343996419,0.7446075157,1.3591394566 \backslash \mathrm{C}, 0.7783710941,-2$. $3496431305,1.41788549 \backslash \mathrm{C}, 2.9621046993,1.074070672,-1.1418375507 \backslash \mathrm{H}, 2.461$ $8445103,0.9971871265,-2.112170483 \backslash \mathrm{H}, 0.4186209348,-2.3487004165,-2.0543$ $385921 \backslash \mathrm{H}, 3.2410667632,2.1179135782,-0.9761501592 \backslash \mathrm{H},-0.2356978467,-3.49$ $86524887,-0.8693786852 \backslash \mathrm{H}, 3.8682693987,0.4668752637,-1.1644534777 \backslash \mathrm{H}, 1.5$ $210208648,-3.3370402684,-1.0720038184 \backslash \mathrm{H}, 2.0813460959,0.4319471952,2.17$ $98729075 \backslash \mathrm{H}, 0.7736614094,-1.5959859597,2.2116564245 \backslash \mathrm{H}, 3.6456082114,0.14$ 
$11166474,1.3809689759 \backslash \mathrm{H}, 1.7222449757,-2.899927783,1.4563708688 \backslash \mathrm{H}, 2.997$ $9470123,1.794826849,1.5184942005 \backslash \mathrm{H},-0.0475515835,-3.0428497168,1.60457$ $78038 \backslash \mathrm{H},-1.9568415769,-2.5142239593,0.0136929483 \backslash 0,-5.3504555264,-0.67$ $15204843,-0.0133500302 \backslash 0,-4.3187872258,-2.5915124024,0.0234704145 \backslash \backslash$ Ver sion=IA64L-G03RevD.01 \State $=2-A \backslash H F=-954.9724905 \backslash S 2=0.753742 \backslash S 2-1=0 . \backslash S 2$ $\mathrm{A}=0.75001 \backslash \mathrm{RMSD}=5.892 \mathrm{e}-09 \backslash \mathrm{RMSF}=3.457 \mathrm{e}-06 \backslash \mathrm{Thermal}=0 . \backslash \mathrm{Dipole}=1.025341,1.5$ $369478,0.2490608 \backslash P G=C 01 \quad[X(C 16 H 17 N 203)] \backslash \backslash @$

\section{5-nitro-TMAO+}

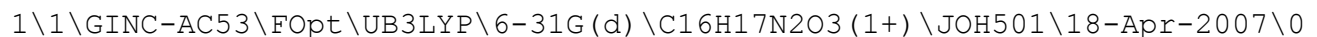

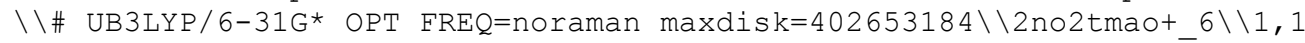
$\backslash \mathrm{N}, 0.0094244234,-0.0020716543,0.0140176779 \backslash 0,1.210926051,-0.031 \overline{3} 775898$ $, 0.0243871345 \backslash \mathrm{C},-2.4256113134,1.3674182775,-0.0002974411 \backslash \mathrm{C},-3.55375245$ $32,2.2502008541,0.0041425404 \backslash C,-4.4028132754,2.2817477446,1.138014848 \backslash$ $\mathrm{C},-3.7897163411,3.0720135016,-1.1279830761 \backslash \mathrm{H},-4.6385914126,3.749309856$ $1,-1.1179474439 \backslash \mathrm{C},-4.1098408771,1.4790947798,2.2130425367 \backslash \mathrm{C},-2.9497254$ $513,3.0181738864,-2.2202388509 \backslash \mathrm{N},-5.0081296307,1.4868866272,3.38853340$ $83 \backslash \mathrm{H},-3.1260045758,3.6579742135,-3.0787295192 \backslash \mathrm{C},-2.9546113297,0.676908$ $6898,2.2657503066 \backslash \mathrm{C},-1.8831091843,2.0944812078,-2.2568400469 \backslash \mathrm{H},-1.2853$ $555835,2.0273369628,-3.1585590224 \backslash \mathrm{C},-2.0982290836,0.6452371123,1.18262$ $69181 \backslash \mathrm{C},-1.6511924154,1.2418386997,-1.1890363128 \backslash \mathrm{C},-0.7151779338,0.034$ $2646539,1.3508356909 \backslash \mathrm{C},-0.7233067853,0.0290313136,-1.3222897564 \backslash \mathrm{C},-0.7$ $216888612,-1.4390936837,1.8520432518 \backslash C,-1.5365400251,-1.2818379121,-1$. $4537447153 \backslash C, 0.1460262432,0.9073130485,2.2956746203 \backslash C, 0.3227319486,0.1$ $299497123,-2.4294712141 \backslash \mathrm{H}, 0.2206789924,1.9312098238,1.9187012384 \backslash \mathrm{H}, 0.9$ $062938188,1.0521139788,-2.364241182 \backslash \mathrm{H},-0.3433445017,0.9359501097,3.270$ $9708763 \backslash \mathrm{H},-0.1870669094,0.1025943285,-3.3949828352 \backslash \mathrm{H}, 1.146868996,0.489$ $7143083,2.4167238004 \backslash \mathrm{H}, 1.0091967988,-0.718853158,-2.394124006 \backslash \mathrm{H},-1.338$ $0411609,-2.0916425616,1.2321263754 \backslash \mathrm{H},-2.3062432059,-1.3795306803,-0.68$ $68187024 \backslash \mathrm{H}, 0.3015851615,-1.8219716655,1.890395586 \backslash \mathrm{H},-0.8793563775,-2.1$ $568933779,-1.4361914098 \backslash \mathrm{H},-1.126010734,-1.4451904504,2.8668879326 \backslash \mathrm{H},-2$ $.0386152533,-1.2475266539,-2.4251859361 \backslash \mathrm{H},-5.276974451,2.9225686325,1$. $1646075974 \backslash \mathrm{H},-2.7566974226,0.1394910592,3.1855403386 \backslash 0,-5.995011745,2$. $210986674,3.3350110923 \backslash 0,-4.6958741397,0.762199331,4.3301476994 \backslash \backslash$ Versi on $=I A 64 \mathrm{~L}-\mathrm{G} 03 \mathrm{RevD} .01 \backslash \mathrm{State}=1-\mathrm{A} \backslash \mathrm{HF}=-954.7145328 \backslash \mathrm{S} 2=0 . \backslash \mathrm{S} 2-1=0 . \backslash \mathrm{S} 2 \mathrm{~A}=0 . \backslash \mathrm{RMS}$ $\mathrm{D}=8.336 \mathrm{e}-09 \backslash \mathrm{RMSF}=7.790 \mathrm{e}-06 \backslash \mathrm{Thermal}=0 . \backslash \mathrm{Dipole}=2.2952448,-0.7659598,-2.4$ $355028 \backslash P G=C 01 \quad[X(C 16 H 17 N 203)] \backslash \backslash e$

\section{5-nitro-TMAO-}

$1 \backslash 1 \backslash G I N C-A C 39 \backslash F O p t \backslash U B 3 L Y P \backslash 6-31 G(d) \backslash C 16 H 17 N 2 O 3(1-) \backslash J O H 501 \backslash 18-A p r-2007 \backslash 0$

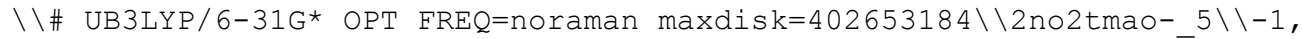
$1 \backslash \mathrm{N},-0.0028731287,0.0009388142,-0.0010497195 \backslash 0,1.2886500795,0.00131806$ $45,-0.0174615071 \backslash \mathrm{C},-0.64623954,1.3486032718,0.018802893 \backslash \mathrm{C},-0.040494059$ $8,2.0849200839,1.2339057106 \backslash C,-2.8407980246,-0.0049981268,-0.060981865$ $8 \backslash C,-4.291192883,-0.0041572915,0.0157055268 \backslash C,-4.9785494461,-1.2389148$ $101,-0.1919172825 \backslash \mathrm{C},-4.992403097,1.181234817,0.2773201892 \backslash \mathrm{H},-6.0650128$ $132,-1.239871218,-0.1342297355 \backslash \mathrm{H},-6.0740718659,1.1841065724,0.33580945$ $97 \backslash \mathrm{C},-4.2876526949,-2.3969537322,-0.4679997109 \backslash \mathrm{C},-4.3004080818,2.39055$ $68199,0.4582723873 \backslash \mathrm{H},-4.8275813348,-3.3285044483,-0.6308147839 \backslash \mathrm{N},-4.98$ $08697899,3.5889236682,0.7107220614 \backslash C,-2.8817958064,-2.3921159265,-0.54$ $0480579 \backslash \mathrm{C},-2.8830916097,2.3944829271,0.3767058025 \backslash \mathrm{H},-2.3611357214,-3.3$ $187025057,-0.7638992279 \backslash \mathrm{C},-2.1598123036,-1.2124492905,-0.331449758 \backslash C_{,}-$ $2.1623467049,1.2503860099,0.1338139371 \backslash C,-0.6409080386,-1.2737090031,-$ $0.4389549489 \backslash \mathrm{C},-0.2066998047,-1.5551528631,-1.9007349822 \backslash \mathrm{C},-0.25004835$ $23,2.0836004347,-1.2860623107 \backslash C,-0.0636503237,-2.3695797079,0.48845955$ $74 \backslash \mathrm{H},-0.4022558375,-2.2093848697,1.5171540553 \backslash \mathrm{H},-0.3436805428,1.592358$ $7257,2.1633070555 \backslash \mathrm{H},-0.3914193471,-3.36257895,0.1688274294 \backslash \mathrm{H},-0.386011$ $4655,3.121004181,1.2664456907 \backslash \mathrm{H}, 1.0274128095,-2.3265358824,0.464506843$ $4 \backslash \mathrm{H}, 1.0493708054,2.0696192154,1.1641571334 \backslash \mathrm{H},-0.6419038689,-0.81714053$ $98,-2.5821013515 \backslash \mathrm{H},-0.713834162,1.6077769322,-2.156376397 \backslash \mathrm{H}, 0.88384665$ $06,-1.5075828785,-1.9732586986 \backslash \mathrm{H}, 0.8381005508,2.0612161223,-1.40391347$ $19 \backslash \mathrm{H},-0.5479082566,-2.5463210449,-2.2161118622 \backslash \mathrm{H},-0.5916304997,3.12262$ $58723,-1.2503744417 \backslash \mathrm{H},-2.4019185692,3.3556274425,0.5120941092 \backslash 0,-6.250$ 
$3386839,3.5626023486,0.7783087213 \backslash 0,-4.3118242376,4.6632607655,0.86309$ $40716 \backslash \backslash$ Version=IA64L-G03RevD.01\State $=1-A \backslash H F=-955.0113391 \backslash \mathrm{S} 2=0.968798 \backslash$ $\mathrm{S} 2-1=0 . \backslash \mathrm{S} 2 \mathrm{~A}=0.121586 \backslash \mathrm{RMSD}=8.252 \mathrm{e}-09 \backslash \mathrm{RMSF}=1.531 \mathrm{e}-05 \backslash \mathrm{Thermal}=0 . \backslash \mathrm{Dipole}=2$ $.3730606,-3.0544088,-0.9508815 \backslash \mathrm{PG}=\mathrm{C} 01 \quad[\mathrm{X}(\mathrm{C} 16 \mathrm{H} 17 \mathrm{~N} 2 \mathrm{O} 3)] \backslash \backslash \mathrm{Q}$

\section{6-nitro-TMAO• (54)}

$1 \backslash 1 \backslash G I N C-A C 10 \backslash F O p t \backslash U B 3 L Y P \backslash 6-31 G(d) \backslash C 16 H 17 N 2 O 3$ (2) \JOH501\19-Apr-2007\0

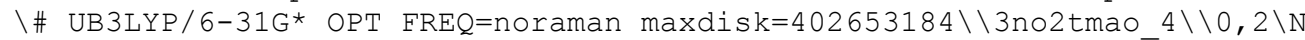
$,-0.0011580854,-0.0062809091,-0.0067858517 \backslash 0,1.2829210085,-0.005401932$ $6,0.0010909479 \backslash C,-0.6373657891,1.339956202,0.0079548687 \backslash C,-0.204177971$ $9,2.0975949825,-1.2723247925 \backslash C,-2.8575130184,0.0074476624,-0.056201161$ $6 \backslash C,-4.3029116088,-0.015541165,0.0443599563 \backslash C,-4.9857408629,-1.2468810$ $387,-0.1280517689 \backslash \mathrm{C},-4.9631510091,1.233879497,0.2594283795 \backslash \mathrm{H},-6.062622$ $4692,-1.2708780689,-0.054334352 \backslash \mathrm{N},-6.4229421068,1.3466893063,0.4164641$ $929 \backslash \mathrm{C},-4.2824791237,-2.3984149178,-0.3891671483 \backslash \mathrm{C},-4.2684800852,2.4195$ $164934,0.3339699804 \backslash \mathrm{H},-4.8156471391,-3.3343811605,-0.5297250677 \backslash \mathrm{H},-4.8$ $147267338,3.3443853678,0.4708608099 \backslash \mathrm{C},-2.8789432695,-2.3821795054,-0.4$ $747256573 \backslash \mathrm{C},-2.8705893934,2.416865965,0.2523796496 \backslash \mathrm{H},-2.3605802485,-3$. $3118590514,-0.6843175882 \backslash \mathrm{H},-2.350064922,3.364194666,0.3372421645 \backslash \mathrm{C},-2$. $1593259317,-1.2140953944,-0.3086818899 \backslash C,-2.1562632385,1.2443182112,0$. $0770138823 \backslash C,-0.6428244819,-1.2728516908,-0.4601639905 \backslash C,-0.2605242816$ $,-1.5078521937,-1.9429102351 \backslash C,-0.041148084,-2.3908701,0.4210827726 \backslash C$, $-0.080842032,2.0583814886,1.2597208758 \backslash \mathrm{H},-0.357621395,-2.272420791,1.4$ $619314971 \backslash \mathrm{H},-0.4268083531,1.5637021148,2.1725641141 \backslash \mathrm{H},-0.3565504862,-3$ $.377037196,0.0715464153 \backslash \mathrm{H},-0.4013038532,3.1029157479,1.2871784047 \backslash \mathrm{H}, 1$. $0473390664,-2.334346514,0.3753876992 \backslash \mathrm{H}, 1.009407043,2.023639192,1.23262$ $28799 \backslash \mathrm{H},-0.7129225091,-0.7530569281,-2.5938393518 \backslash \mathrm{H},-0.6244054731,1.63$ $2619804,-2.1694064886 \backslash \mathrm{H}, 0.8270133818,-1.4601575125,-2.0453760446 \backslash \mathrm{H}, 0.8$ $86395944,2.0801959932,-1.341560921 \backslash \mathrm{H},-0.6081333898,-2.4908953749,-2.27$ $47702766 \backslash \mathrm{H},-0.5431788646,3.1373395338,-1.2385775483 \backslash 0,-7.046819048,0.3$ $933541916,0.8872525477 \backslash 0,-6.9432511852,2.4160850249,0.0944680958 \backslash \backslash$ Vers ion $=I A 64 \mathrm{~L}-\mathrm{G} 03 \mathrm{RevD} .01 \backslash$ State $=2-\mathrm{A} \backslash \mathrm{HF}=-954.9650625 \backslash \mathrm{S} 2=0.753737 \backslash \mathrm{S} 2-1=0 . \backslash \mathrm{S} 2 \mathrm{~A}$ $=0.75001 \backslash \mathrm{RMSD}=5.588 \mathrm{e}-09 \backslash \mathrm{RMSF}=3.159 \mathrm{e}-06 \backslash \mathrm{Thermal}=0 . \backslash \mathrm{Dipole}=0.9738549,-0$. $3384456,-0.4405384 \backslash \mathrm{PG}=\mathrm{C} 01 \quad[\mathrm{X}(\mathrm{C} 16 \mathrm{H} 17 \mathrm{~N} 2 \mathrm{O} 3)] \backslash \backslash @$

\section{6-nitro-TMAO+}

$1 \backslash 1 \backslash G I N C-A C 17 \backslash F O p t \backslash U B 3 L Y P \backslash 6-31 G(d) \backslash C 16 H 17 N 2 O 3(1+) \backslash J O H 501 \backslash 19-A p r-2007 \backslash 0$

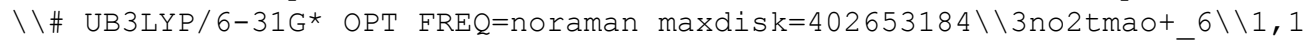
$\backslash \mathrm{N},-0.001228075,-0.0045011565,0.008287058 \backslash 0,1.2009971883,-0.029 \overline{3} 396419$ $, 0.0273833042 \backslash \mathrm{C},-2.416182415,1.4090774451,-0.023486298 \backslash \mathrm{C},-3.5241826036$ $, 2.3246484699,-0.035004058 \backslash \mathrm{C},-4.3825652898,2.3026475836,1.1052912718 \backslash \mathrm{C}$ $,-3.7009285258,3.1554435334,-1.1751753498 \backslash \mathrm{H},-4.5179223286,3.8627903223$ $,-1.1922275268 \backslash \mathrm{C},-4.1609974858,1.4637292342,2.1733253349 \backslash \mathrm{C},-2.83146036$ $66,3.0774930975,-2.2429461739 \backslash \mathrm{H},-4.8630267246,1.4593245508,2.998287348$ $3 \backslash \mathrm{H},-2.974653014,3.7278120259,-3.0998391587 \backslash \mathrm{C},-3.0023189701,0.66828334$ $98,2.2128090192 \backslash \mathrm{C},-1.7888837902,2.1304787701,-2.2649052009 \backslash \mathrm{H},-1.171914$ $601,2.0510838183,-3.1522844621 \backslash C,-2.1167560653,0.6619866279,1.15032018$ $83 \backslash \mathrm{C},-1.6164095809,1.2666512204,-1.1977107998 \backslash \mathrm{C},-0.7373317799,0.046818$ $8192,1.3359549331 \backslash C,-0.7308446185,0.0207486132,-1.3245254648 \backslash C,-0.7491$ $375585,-1.4213746117,1.8500657164 \backslash \mathrm{C},-1.5830900597,-1.2649793975,-1.442$ $609939 \backslash C, 0.1139344423,0.9297274065,2.2810427377 \backslash C, 0.3167624255,0.07772$ $8159,-2.4335437227 \backslash \mathrm{H}, 0.1940594516,1.9492474907,1.8935788609 \backslash \mathrm{H}, 0.924054$ $6832,0.9852294801,-2.3844212914 \backslash \mathrm{H},-0.3841886844,0.9702394971,3.2514734$ $303 \backslash \mathrm{H},-0.1928308024,0.044954322,-3.3989499714 \backslash \mathrm{H}, 1.1130770531,0.5122106$ $062,2.4161562176 \backslash \mathrm{H}, 0.9819265244,-0.7872214722,-2.3809078009 \backslash \mathrm{H},-1.36656$ $72557,-2.0779778954,1.2352470096 \backslash \mathrm{H},-2.3539950448,-1.3329875516,-0.6738$ $989369 \backslash \mathrm{H}, 0.2729379058,-1.8071778985,1.8909532462 \backslash \mathrm{H},-0.9521588383,-2.15$ $89331249,-1.4185493623 \backslash \mathrm{H},-1.1507707161,-1.4206977054,2.865823651 \backslash \mathrm{H},-2$. $086463662,-1.2242678477,-2.4130976808 \backslash \mathrm{N},-5.5694935099,3.182843085,1.21$ $39132701 \backslash \mathrm{H},-2.8010366198,0.0964319199,3.1128002357 \backslash 0,-5.5137781834,4.2$ $90588604,0.684923987 \backslash 0,-6.5185225044,2.746790251,1.8550363783 \backslash \backslash$ Version $=I A 64 \mathrm{~L}-\mathrm{G} 03 \mathrm{RevD} .01 \backslash \mathrm{State}=1-\mathrm{A} \backslash \mathrm{HF}=-954.7072653 \backslash \mathrm{S} 2=0 . \backslash \mathrm{S} 2-1=0 . \backslash \mathrm{S} 2 \mathrm{~A}=0 . \backslash \mathrm{RMSD}=$ $3.544 \mathrm{e}-09 \backslash \mathrm{RMSF}=1.060 \mathrm{e}-05 \backslash \mathrm{Thermal}=0 . \backslash \mathrm{Dipole}=2.6908714,-2.1837099,-0.613$ 


\section{6-nitro-TMAO-}

$1 \backslash 1 \backslash G I N C-A C 28 \backslash F O p t \backslash U B 3 L Y P \backslash 6-31 G(d) \backslash C 16 H 17 N 203$ (1-) \JOH501 \21-Apr-2007 0

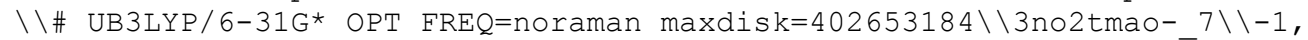
$1 \backslash \mathrm{N},-0.0858418031,-0.2087290601,0.0721016155 \backslash 0,0.9429638915,-0 . \overline{9} 833361$ $012,0.0799150317 \backslash \mathrm{C},-2.4088975775,1.4744305261,0.0606259614 \backslash \mathrm{C},-3.529812$ $4118,2.4065131043,0.07645759 \backslash \mathrm{C},-4.1041842314,2.7405228833,1.322977304 \backslash$ $\mathrm{C},-4.0064337355,2.9336603489,-1.19307129 \backslash \mathrm{N},-5.0668795666,3.8490154795$, $-1.3335811917 \backslash \mathrm{C},-3.6320866157,2.1971263438,2.5085788147 \backslash \mathrm{C},-3.386647579$ $5,2.5079871446,-2.3882527112 \backslash \mathrm{H},-4.1003367346,2.4696770926,3.4522567551$ $\backslash \mathrm{H},-3.7717344153,2.9043954686,-3.3173614826 \backslash \mathrm{C},-2.5592977644,1.30330002$ $89,2.4952559152 \backslash \mathrm{C},-2.3264275335,1.6226930628,-2.3665769736 \backslash \mathrm{H},-1.889245$ $7725,1.3267751665,-3.3178471393 \backslash \mathrm{C},-1.9419626869,0.9437678307,1.3017000$ $261 \backslash \mathrm{C},-1.8035510931,1.099029396,-1.1756843425 \backslash \mathrm{C},-0.7980886453,-0.06492$ $07472,1.3742242822 \backslash \mathrm{C},-0.64344487,0.120347252,-1.2709735538 \backslash \mathrm{C},-1.334263$ $8154,-1.4639040448,1.7705811774 \backslash \mathrm{C},-1.0898406328,-1.2092514534,-1.93320$ $10435 \backslash \mathrm{C}, 0.2790646763,0.3853496344,2.388659521 \backslash \mathrm{C}, 0.5333320917,0.7235167$ $966,-2.0751613086 \backslash \mathrm{H}, 0.6300973097,1.392784416,2.1432133148 \backslash \mathrm{H}, 0.84377025$ $7,1.6743785208,-1.630405452 \backslash \mathrm{H},-0.1259113461,0.4015709793,3.4039746841 \backslash$ $\mathrm{H}, 0.234258353,0.9124086509,-3.1097311088 \backslash \mathrm{H}, 1.1256498445,-0.3038009436$, $2.3527753178 \backslash \mathrm{H}, 1.3810693127,0.0340189903,-2.067786794 \backslash \mathrm{H},-2.1215958197$, $-1.7842353168,1.0806888993 \backslash \mathrm{H},-1.932716026,-1.6464554589,-1.3883265021 \backslash$ $\mathrm{H},-0.5188508527,-2.1936378845,1.7465756819 \backslash \mathrm{H},-0.2598216567,-1.92292676$ $81,-1.9400296048 \backslash \mathrm{H},-1.7631549486,-1.4330286625,2.7773777635 \backslash \mathrm{H},-1.41643$ $15989,-1.0250806233,-2.9617234199 \backslash \mathrm{H},-4.9322835401,3.432795726,1.315761$ $454 \backslash \mathrm{H},-2.2090101856,0.881818826,3.4345724732 \backslash 0,-5.6907341128,4.3021732$ $464,-0.3204436946 \backslash 0,-5.3915681643,4.2214001491,-2.5068959699 \backslash \backslash$ Version= IA 64L-G03RevD. $01 \backslash$ State $=1-A \backslash H F=-955.0061533 \backslash S 2=1.017689 \backslash S 2-1=0 . \backslash S 2 A=0.1$ $49312 \backslash \mathrm{RMSD}=5.653 e-09 \backslash \mathrm{RMSF}=4.033 e-06 \backslash \mathrm{Thermal}=0 . \backslash \mathrm{Dipole}=2.6140419,-2.632$ $1254,1.1267295 \backslash \mathrm{PG}=\mathrm{C} 01 \quad[\mathrm{X}(\mathrm{C} 16 \mathrm{H} 17 \mathrm{~N} 2 \mathrm{O} 3)] \backslash \backslash @$ 